\title{
Evaluation von \\ Forschung und Lehrprogrammen \\ an den \\ Fachbereichen für Mathematik der österreichischen Universitäten
}

Endbericht der Evaluierungskommission

Im Auftrag der Österreichischen Mathematischen Gesellschaft

Finanziert vom Bundesministerium für Bildung, Wissenschaft und Kultur; Wien 


\section{Inhalt}

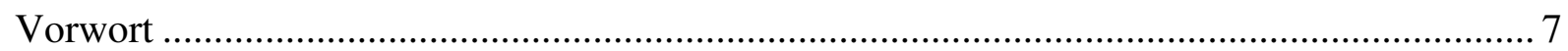

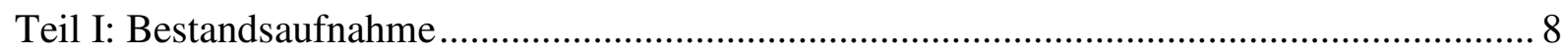

1. Zum Ausbaustand der mathematischen Fachbereiche an österreichischen Universitäten ..... 8

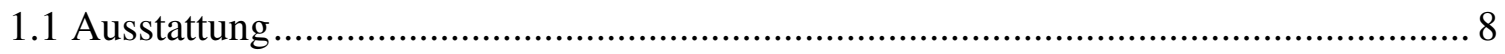

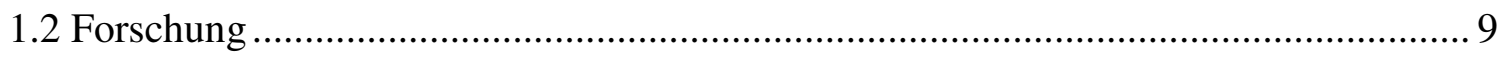

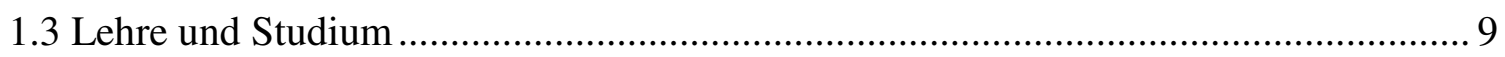

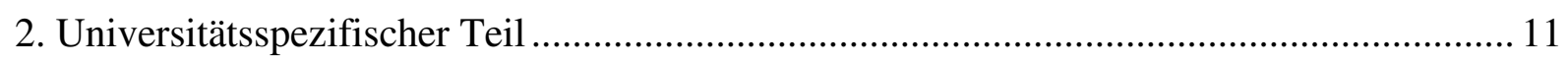

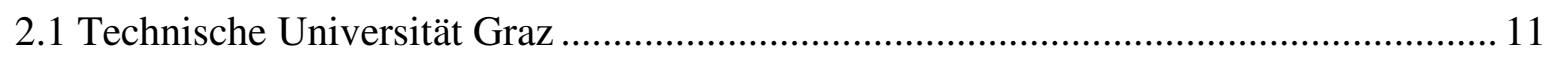

Teil A: Forschung, Wissenschaftlicher Nachwuchs ..................................................... 11

Teil B: Lehre und Studium am Fachbereichs Mathematik an der TU Graz ................... 22

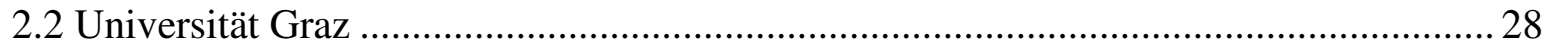

Teil A: Forschung, Wissenschaftlicher Nachwuchs ................................................. 28

Teil B: Lehre und Studium am Institut für Mathematik und wissenschaftliches Rechnen

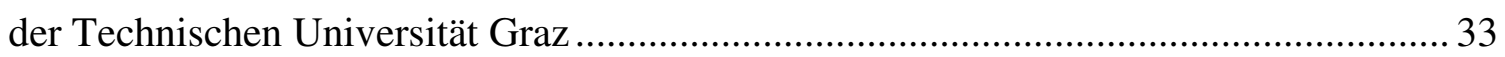

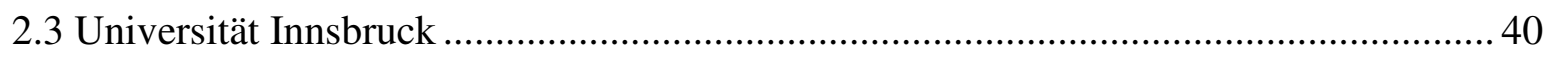

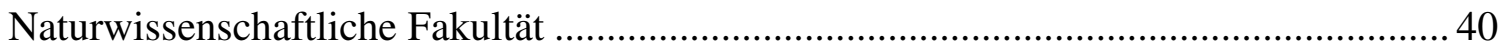

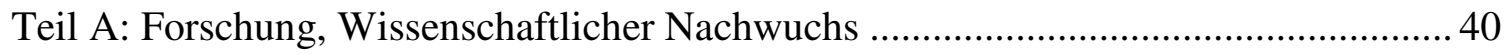

Teil B: Lehre und Studium am Fachbereich Mathematik der Universität Innsbruck ...... 45

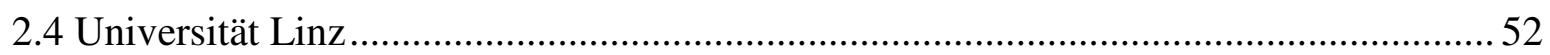

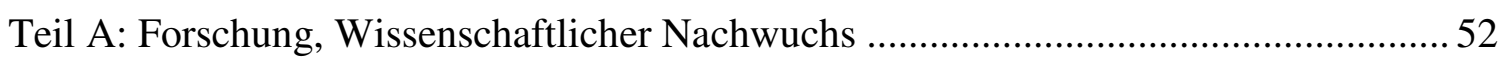

Teil B: Lehre und Studium am Fachbereich Mathematik der Universität Linz............... 66

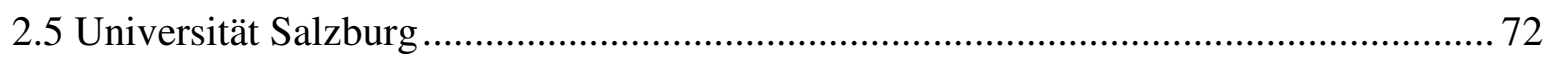

Teil A: Forschung, Wissenschaftlicher Nachwuchs .................................................. 72

Teil B: Lehre und Studium am Fachbereich Mathematik der Universität Salzburg ........ 75

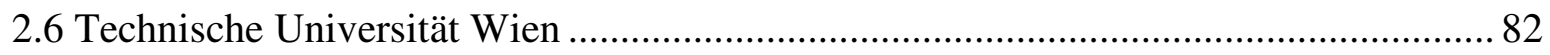

Fakultät für Mathematik und Geoinformation ........................................................... 82

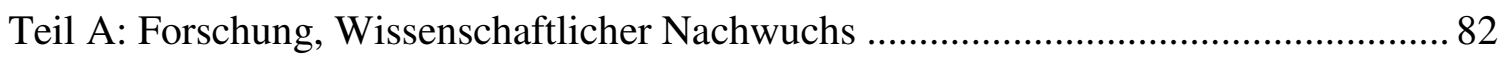

Teil B: Lehre und Studium an der Fakultät für Mathematik und Geoinformation der TU

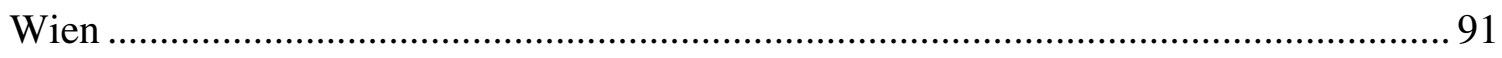

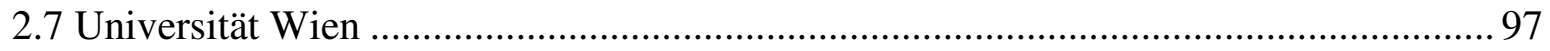

Teil A: Forschung, Wissenschaftlicher Nachwuchs ..................................................... 97

Teil B: Lehre und Studium an der Fakultät für Mathematik der Universität Wien ....... 101

II. Empfehlungen. 
1. Eine erweiterte Rolle der Mathematik

1.1 Mathematik: Eine Wissenschaft mit Erneuerungspotential

1.2 Es besteht Handlungsbedarf: Mathematiker werden fehlen

2. Rahmenbedingungen.

2.1 Unmittelbare Auswirkungen des neuen Universitätsgesetzes.

2.2 Das Personalproblem

2.3 Eine gezielte Institutspolitik gegen die Vereinzelung

2.4 Der Stellenmarkt in der Mathematik

2.5 Aufbau einer adäquaten Personalstruktur hat hohe Priorität.

3. Zur künftigen Struktur der Hochschulmathematik in Österreich

3.1 Zahl der universitären mathematischen Ausbildungsstätten in Österreich

3.2. Anzahl der Fachrichtungen

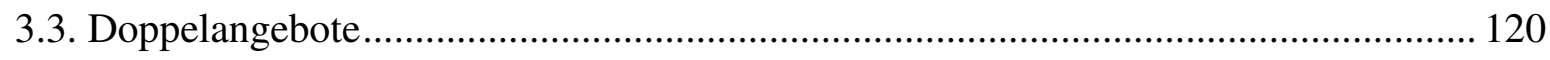

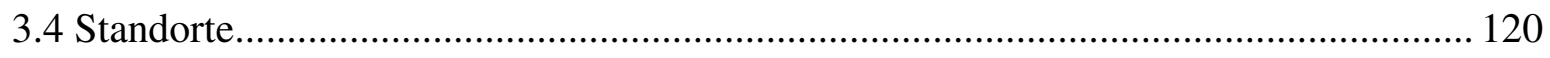

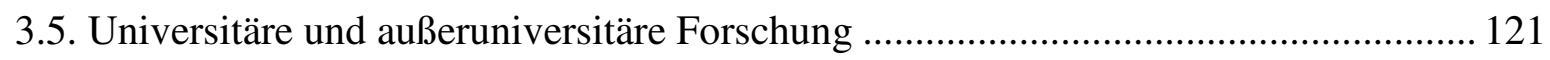

3.6 Zusammenarbeit von universitären und außeruniversitären Forschungseinrichtungen 122

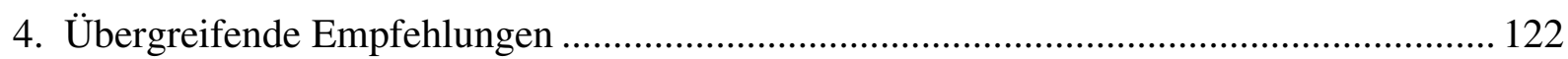

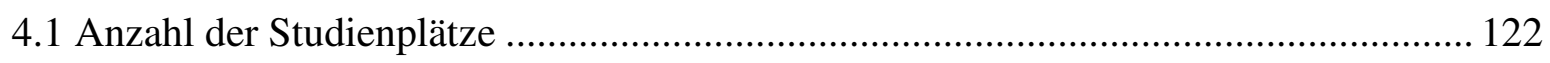

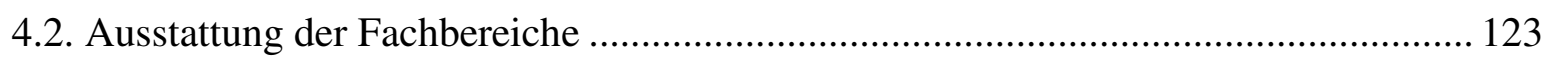

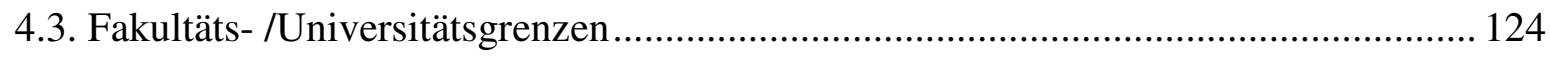

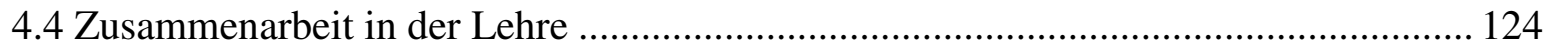

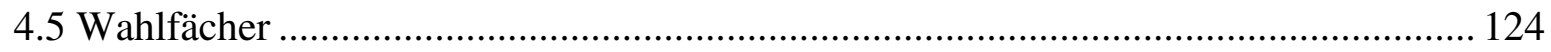

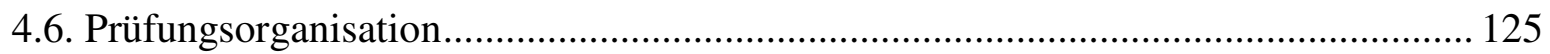

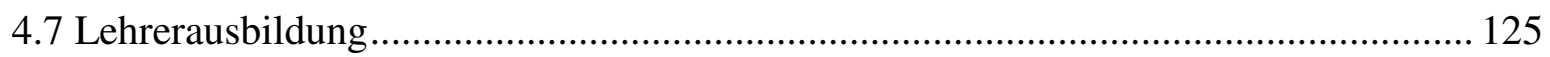

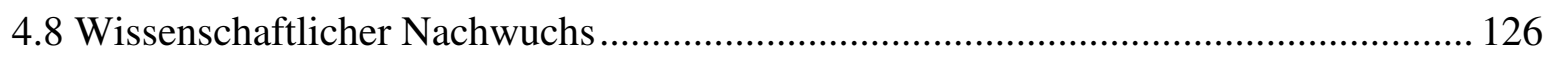

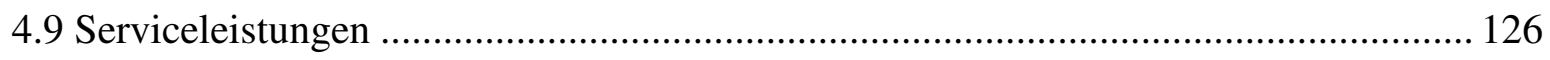

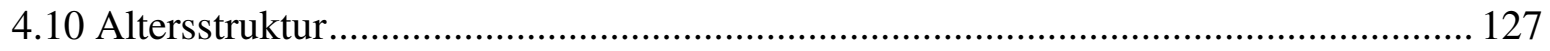

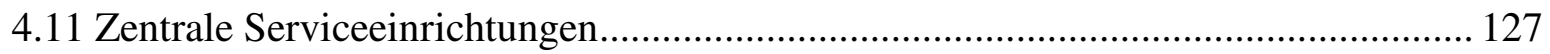

4.12 Wirtschaftliche Komponenten mathematischer Studiengänge ................................ 128

5. Einzelempfehlungen: Zur weiteren Entwicklung der mathematischen Fachbereiche ....... 129

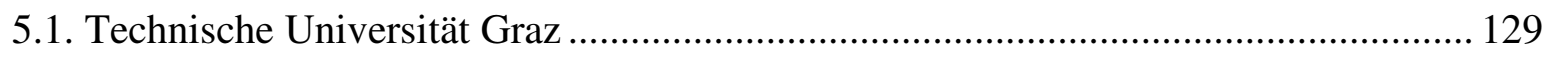

Institut für Mathematik A (Analysis and Computational Number Theory) ................... 129

Institut für Mathematik B (Mathematische Optimierung, dynamische Systeme und diskrete Mathematik) 
Institut für Mathematik C (Mathematische Strukturtheorie)....................................... 131

Institut für Mathematik D (Numerik und Partielle Differentialgleichungen) ................ 131

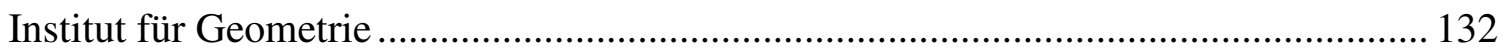

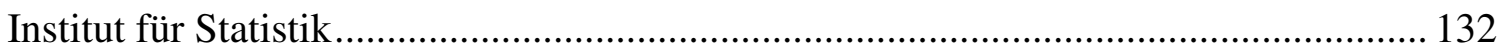

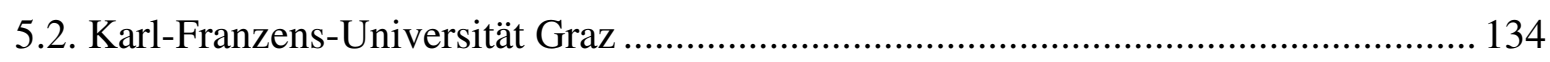

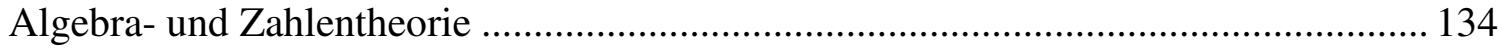

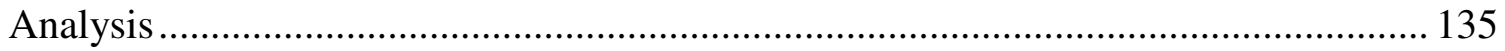

Kontrolltheorie, Optimierung und Wissenschaftliches Rechnen, Scientific Computing135

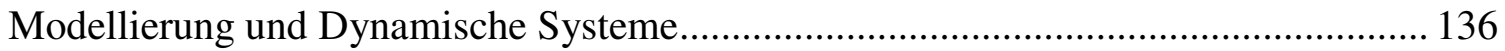

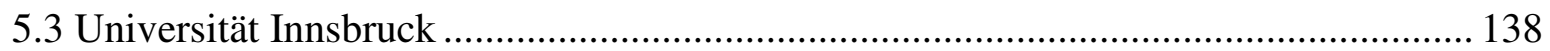

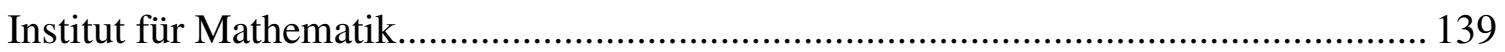

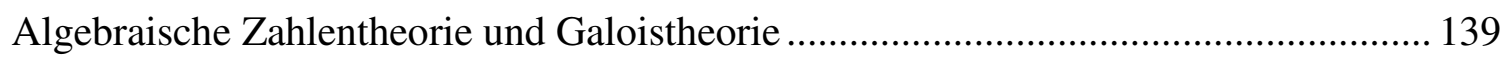

Dynamische Systeme und Funktionalgleichungen ..................................................... 139

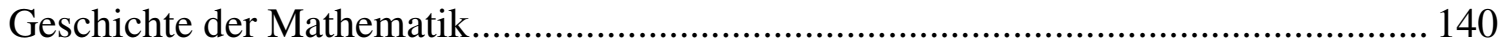

Jordan-Theorie und Steinberg-Gruppen ................................................................... 140

Lineare Systemtheorie und Computeralgebra ........................................................... 140

Institut für Technische Mathematik, Geometrie und Bauinformatik ............................. 141

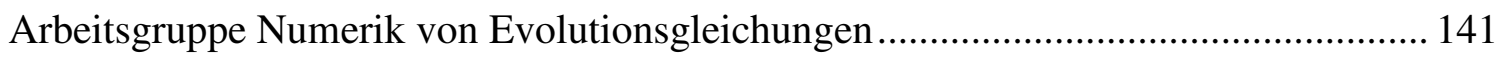

Arbeitsgruppe Nichtlineare Analysis/Unscharfe Daten ............................................ 141

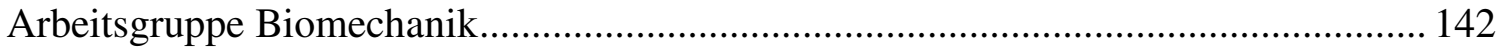

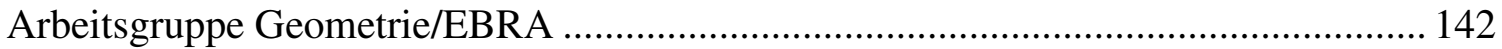

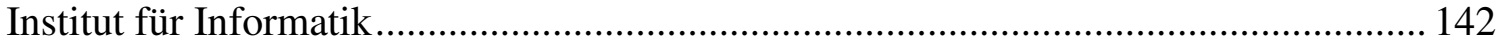

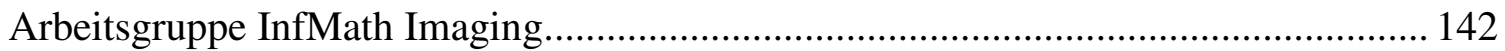

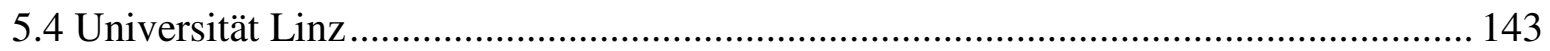

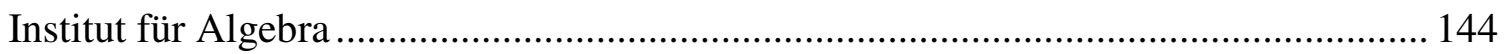

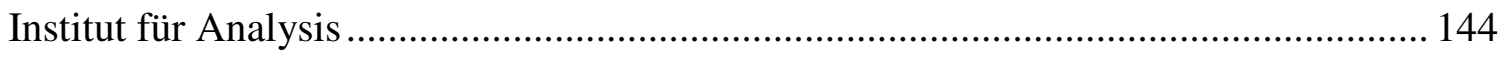

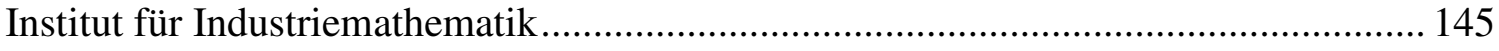

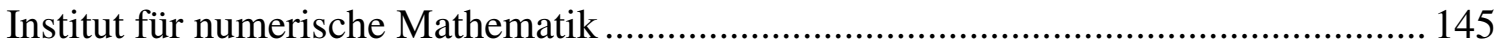

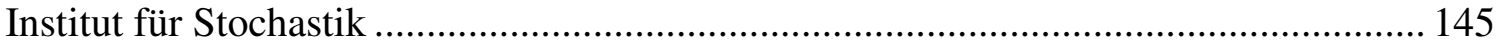

Institut für symbolisches Rechnen (

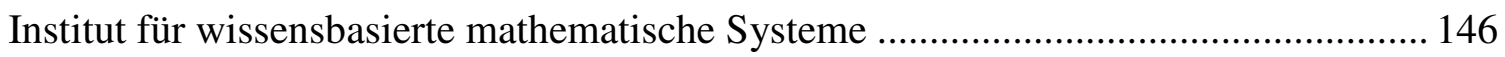

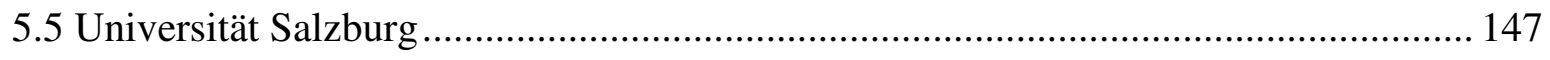

Arbeitsgruppe Diskrete Mathematik und Anwendungen .......................................... 148

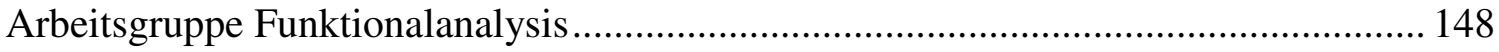


Arbeitsgruppe Geometrie und Versicherungsmathematik ........................................ 148

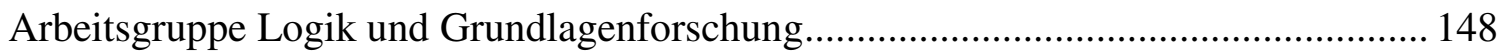

Arbeitsgruppe Monte Carlo- und Quasi-Monte Carlo-Methoden ................................... 149

Arbeitsgruppe Numerische Mathematik und Approximationstheorie ........................... 149

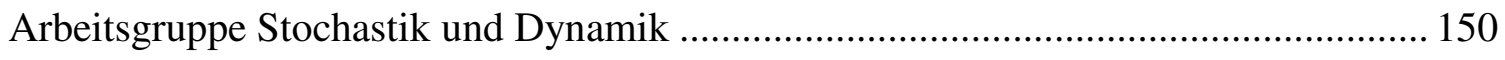

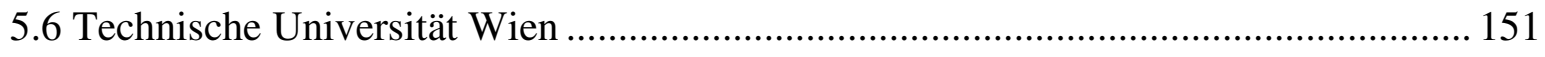

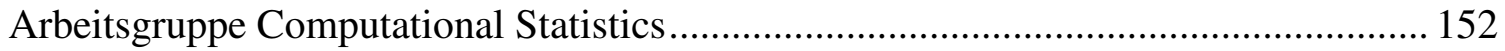

Arbeitsgruppe Computational Neurosciences ............................................................. 152

Arbeitsgruppe Simulation von Differentialgleichungen ............................................. 153

Arbeitsgruppe Partielle Differentialgleichungen und dynamische Systeme.................. 153

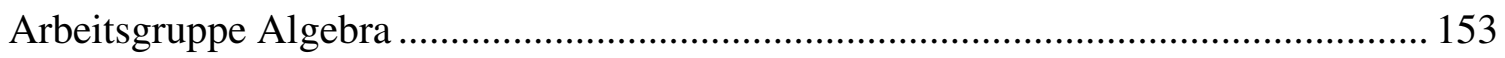

Arbeitsgruppe Differentialgeometrie und Geometrische Strukturen ............................ 154

Arbeitsgruppe Kombinatorik und Algorithmen ........................................................ 154

Arbeitsgruppe Konvexe und Diskrete Geometrie ....................................................... 154

Arbeitsgruppe Geometrische Modellierung und Industrielle Geometrie ....................... 155

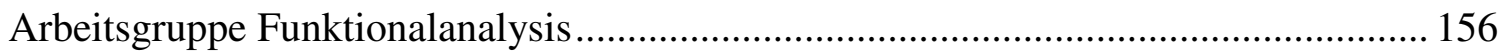

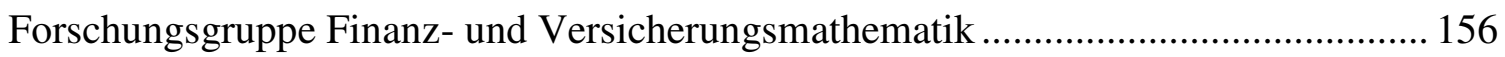

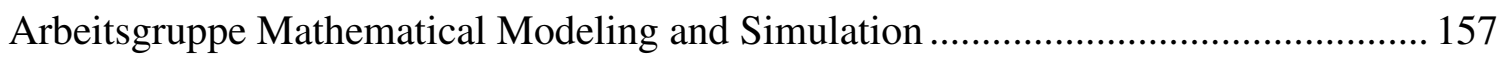

Arbeitsgruppe Mathematische Strukturanalyse und Interpolation ............................... 157

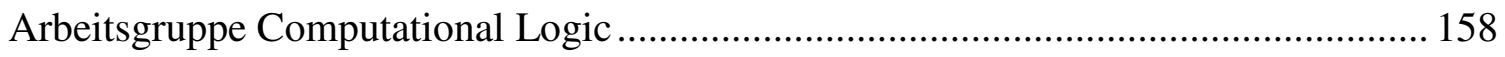

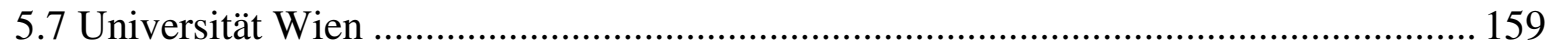

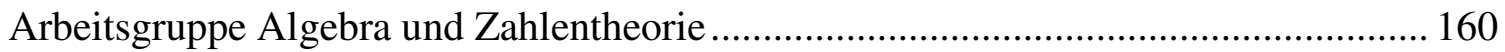

Arbeitsgruppe Partielle Differentialgleichungen ........................................................... 160

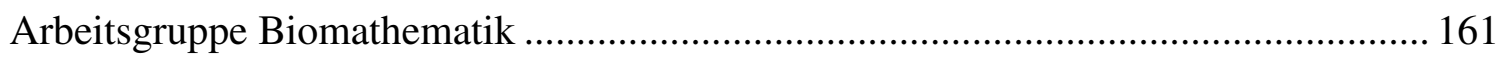

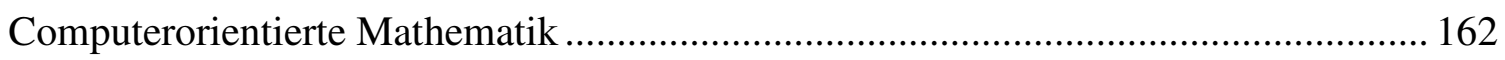

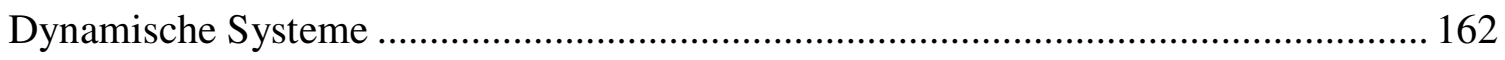

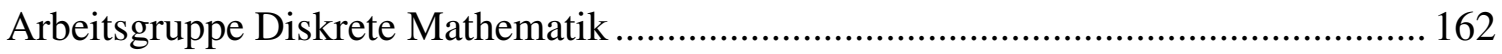

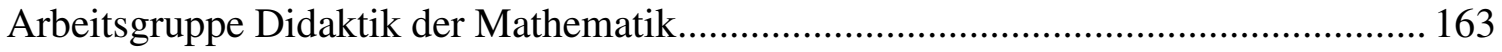

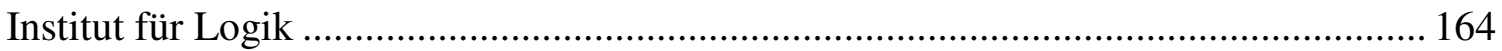

Institut für Analysis, Differentialgleichungen und Topologie .................................... 164

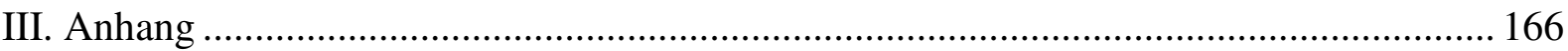

Tabellarische Zusammenstellung der Ausstattung mathematischer Fachbereiche an den

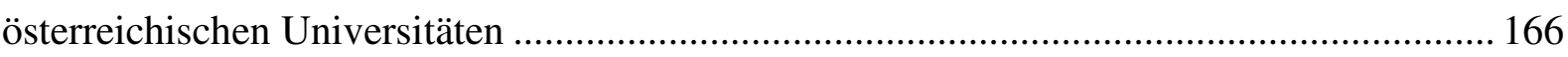

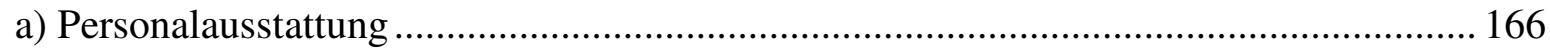




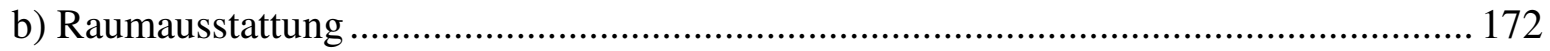

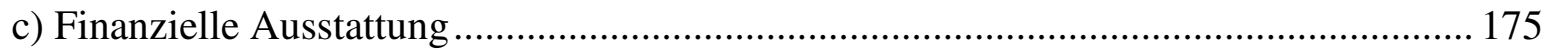

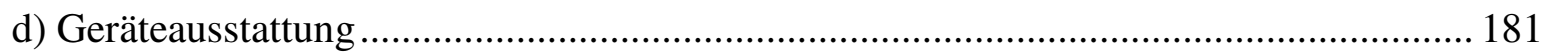

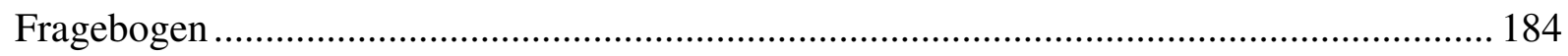

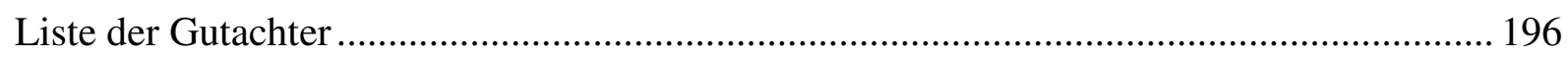




\section{Vorwort}

Im Sommer 2004 war die Österreichische Mathematische Gesellschaft (ÖMG) mit der Bitte an die Deutsche Mathematiker-Vereinigung (DMV) herangetreten, bei der Evaluation von Lehre und Forschung an mathematischen Fachbereichen der österreichischen Universitäten Hilfestellung zu leisten. Die DMV hat daraufhin die Professoren Dr. K.-H. Hoffmann (Angewandte Mathematik) und Dr. J.-P. Bourguignon (Reine Mathematik) gebeten, als Vorsitzende einer international zusammengesetzten Evaluierungskommission diese Arbeit zu übernehmen. Auf Vorschlag der beiden Vorsitzenden wurde die Kommission aus einer Mathematikerin und Mathematikern aus Deutschland, Finnland, der Niederlande, der Schweiz und der USA gebildet. Mit Ausnahme der Universität Klagenfurt nahmen alle österreichischen Universitäten, an denen ein Hauptfachstudium Mathematik eingerichtet ist, auf freiwilliger Basis an der Evaluation teil.

Die Evaluation fand in zwei Abschnitten statt. Zunächst erhielten die Fachbereiche 2004 einen Fragebogen, der einerseits eine Hilfestellung zur Selbstevaluation geben sollte und andererseits der Kommission die benötigten Fakten (vgl. u.a. Anhang und Teil I) zur Verfügung stellte. Nach dem Rücklauf der Fragebögen im Herbst 2004 wurde die Evaluation vor Ort von den Vorsitzenden vorbereitet. Danach wurden im Januar und Februar 2005 alle teilnehmenden mathematischen Fachbereiche von der Kommission besucht und in ausführlichen Diskussionen zu ihren Vorstellungen in Lehre und Forschung gehört.

Nach einem internen Treffen der Kommission im März 2005 liegt nunmehr der vollständige Bericht vor. Er besteht aus drei Teilen: einer Bestandsaufnahme, die mit den Fachbereichen abgestimmt wurde und der Darstellung der Fachbereiche entspricht, einem Bewertungsteil mit der Einschätzung der Kommission, und im Anhang einem tabellarischen Teil einschließlich des zugrundeliegenden Fragebogens.

Der Gesamtbericht wurde im Juni 2005 den Auftraggebern (ÖMG und Bundesministerium für Bildung, Wissenschaft und Kultur) übergeben.

Die Vorsitzenden der Kommission danken den beteiligten Fachbereichen für das große Engagement bei der Vorbereitung und Durchführung der Evaluation. Unser Dank gilt insbesondere den Mitgliedern der Kommission, die trotz winterlicher Widrigkeiten die Reise durch Österreich zu den Standorten der Fachbereiche nicht gescheut haben.

Wir hoffen, dass die erarbeiteten Unterlagen für alle Beteiligten hilfreich sein werden.

Im Juni 2005

K.-H. Hoffmann

(Forschungszentrum caesar, Bonn)
J.-P. Bourguignon

(l'Institut des Hautes Ètudes Scientifiques, Bures-sur-Yvette) 


\section{Teil I: Bestandsaufnahme}

\section{Zum Ausbaustand der mathematischen Fachbereiche an österreichischen Uni- versitäten}

\subsection{Ausstattung}

Nach Aussage der Fachbereiche ist die Raumsituation an fast allen Hochschulen entspannt. Eine Ausnahme bilden die Wiener Universitäten, die einen erhöhten Raumbedarf zu verzeichnen haben. Alle mathematischen Fachbereiche bemängeln das Fehlen von Verfügungsflächen für drittmittelfinanzierte Forschungsprojekte und Gastforscher.

Die Ausstattung mit PCs und Arbeitsplatzrechnern wird von den Instituten in der Regel als adäquat bezeichnet. In einigen Fällen scheint diese Infrastruktur allerdings veraltet zu sein. Ein Erneuerungsprogramm wird von einzelnen Fachbereichen als vordringlich angesehen. Für mathematische Forschung und Lehre ist eine gute Bibliotheksausstattung eine grundlegende Voraussetzung. Die Fachbereiche bestätigen, dass in der Vergangenheit das hohe Niveau der Literaturversorgung ein Pluspunkt der österreichischen Universitäten darstellte. Durch finanzielle Engpässe bedingt, ist nach Meinung der mathematischen Fachbereiche die ausreichende Literaturversorgung in Gefahr. Notmaßnahmen, die verschiedene Institute ergriffen haben, stoßen auf ihre Grenzen.

Das Hauptproblem für eine zukünftige erfolgreiche und international konkurrenzfähige Mathematik in Österreich sehen gleichlautend alle Fachbereiche in der personellen Ausstattung und in der Personalstruktur ihrer Einrichtung. Die Fachbereiche beklagen die unzureichende Zahl an Qualifikationsstellen für den wissenschaftlichen Nachwuchs, was zur Abwanderung hervorragender junger Wissenschaftler ins Ausland und in die Wirtschaft führe. Die erschwerte Wiederbesetzung b ei den wenigen freiwerdenden Stellen führe zu einem Aderlass der österreichischen Mathematik. Nachbesetzungen von Professoren - aber auch von Mitarbeiterstellen dauern nach Meinung der Fachbereiche zu lange und setzen die österreichischen Hochschulen gegenüber den Mitkonkurrenten um die besten Köpfe in Europa in eine nachrangige Position. Die Gründe hierfür seinen vielschichtig. Jedoch sei erkennbar, dass die großen Universitäten aus verschiedenen Gründen besonders im Nachteil seien. 


\subsection{Forschung}

Die Fachbereiche verweisen auf vielfältige Kooperationen mit in- und ausländischen Hochschulen sowie auf zahlreiche Publikationen und Anerkennungen von wissenschaftlichen Leistungen durch Preisverleihungen und Auszeichnungen. Die Kooperationen beziehen sich schwerpunktmäßig auf Forschungseinrichtungen in USA und in der EU, wobei die Beziehungen zu osteuropäischen Ländern betont werden. Die Anzahl der wissenschaftlichen Publikationen ist ein Indikator für die Aktivität eines Fachbereiches in der Forschung. Die Publikationstätigkeiten der einzelnen Arbeitsgruppen in den Fachbereichen weisen deutliche Unterschiede auf. Das reflektiert auch die Aktualität der Forschungsthemen, die jeweils behandelt werden.

Die Doktoranden und Habilitanden der Fachbereiche sind in vielen Fällen wesentliche Träger der Forschung. Allerdings ist die Promotionszeit mit meist weit mehr als 3 Jahren an den Fachbereichen sehr lang. Entsprechend ist an einigen Hochschulen die Zahl der Promovierenden gering. Das Alter der Habilitanden mit im Schnitt zwischen 35 und 40 Jahren und einer Dauer in einem Fall von 10 Jahren liegt weit über dem Üblichen. Die Fachbereiche verweisen als Begründung auf die unklare Situation des wissenschaftlichen Nachwuchses und auf die derzeitig fehlenden beruflichen Perspektiven hin.

Fast alle mathematischen Fachbereiche pflegen über die Teilnahme an Spezialforschungsbereichen oder anderen gesamtösterreichischen Aktivitäten nach eigenen Angaben enge Kontakte zu den anderen Universitäten. Die wissenschaftlich aktiven Gruppen sind in europäische Netzwerke eingebunden.

\subsection{Lehre und Studium}

Alle mathematischen Fachbereiche bieten Curricula an, die sich in der Fokussierung durchaus unterscheiden. Die Belastung der Lehrenden, abhängig von ihrem jeweiligen Anstellungsvertrag, ist nach Aussage der Fachbereiche unterschiedlich. Doktoranden und Habilitanden seien in der Regel in die Lehre eingebunden. Didaktische Bezüge würden in die aktuelle Lehre integriert. Die Lehre ist nach Meinung der Fachbereiche gut organisiert und über den Studiendekan organisiert. Allgemein beklagt wird die Häufigkeit. Prüfungen wiederholen zu dürfen. 
Die angebotenen Studiengänge sind nach Meinung der Fachbereiche in der dafür vorgesehenen Studienzeit auch studierbar. Die Betreuung der Studierenden sei in ausreichender Form gewährleistet. Die Studenten hätten Möglichkeiten, den Wahlteil des Studiums mit zu gestalten, wenn sie sich entsprechend artikulieren. 


\section{Universitätsspezifischer Teil}

\subsection{Technische Universität Graz}

\section{Teil A: Forschung, Wissenschaftlicher Nachwuchs}

\section{Institut für Mathematik A (Analysis und Computational Number Theory)}

Raum-, Sach-, Personalausstattung

Das Institut verfügt über 10 Räume auf einer Gesamtfläche von $228,80 \mathrm{~m}^{2}$, gute Computerausstattung und eine gute Bibliothek. Über folgende Finanzmittel verfügt das Institut:

\begin{tabular}{|l|r|r|r|}
\hline in $€$ & 2001 & 2002 & 2003 \\
\hline laufende Ausgaben & 15.299 & 10.590 & 10.300 \\
\hline Investitionen (Bundesmittel) & 3.633 & 4.200 & 4.800 \\
\hline Einnahmen Bundesmittel & 13.735 & 12.000 & 9.450 \\
\hline Drittmittel FWF & 249.135 & 242.000 & 257.000 \\
\hline
\end{tabular}

Das Institut beurteilt seine personelle Ausstattung als angemessen. Es hat 10,5 Stellen (2 Professuren, 6 wiss. Personal (1 auf Zeit), 1 Lehrbeauftragter, 1,5 nichtwiss. Personal), die aus Haushaltsmitteln finanziert werden und 6,5 Stellen drittmittelfinanziert. Es gibt keine Abweichungen zwischen Stellen-Soll und -Ist. Die infrastrukturelle Ausstattung bei Bibliothek und Computern wird als gut beurteilt. Es wird jedoch bemängelt, dass Räume für Drittmittelbeschäftigte und Diplomanden fehlen und dass das Reisebudget zu niedrig ist.

\section{Besondere Aktivitäten in der Lehre}

Die Vorstellungen für ein Karrieremodell sind:

1. FWF-Projektstellen; 2. temporäre Mitarbeit auf Haushaltsstellen (Promotion); 3. Auslandsaufenthalt (z. B. Schrödinger-Stipendium); 4. längerfristige Anstellung auf Haushaltsstellen (etwaige Habilitation)

\section{Publikationen}

Das Institut hat zwischen 2001und 200361 Veröffentlichungen in referierten Zeitschriften oder Sammelwerken publiziert. Es wurden 83 Vorträge gehalten, davon 32 auf Einladung. 


\section{Wissenschaftlicher Nachwuchs}

Im Zeitraum 1999-2003 wurden 13 Promotionen abgelegt mit einer durchschnittlichen Dauer von 3,45 Jahren. Das Alter der Doktoranden lag bei 29,66 Jahren. Im gleichen Zeitraum wurden 2 Habilitationen geschrieben mit einer Dauer von 6 Jahren. Das Durchschnittsalter der Habilitanden lag bei 30 Jahren. Doktoranden werden als Tutoren bzw. Studienassistenten in die Lehre einbezogen, Habilitanden als Lehrbeauftragte.

\section{Personalfluktuation}

In den letzten 5 Jahren haben 7 Wissenschaftler das Institut verlassen. An Neuzugängen sind 2 hinzugekommen (eine davon hausintern als Berufungsabwehr, eine externe Besetzung von der TU Wien). Das Institut sieht das Pensionsrecht als Hindernis für externe Berufungen an.

\section{Kooperation in der Forschung}

Das Institut unterhält Forschungskooperationen im Rahmen der Socrates-Programme mit den Universitäten Ostrava, Budapest, Debrecen, Zagreb und in Zukunft mit Marseille (bereits genehmigt). Des weiteren bestehen Kooperationen im Rahmen des START-Projekts und des FSP mit Universitäten in Europa und Übersee (z.B: Marseille, Johannesburg, Beer Sheva (Israel), ETH Zürich, und mehreren österreichischen Universitäten, Universität Leiden, Universität Bordeaux). Darüber hinaus gibt es weitere Kooperationen mit Universitäten in europäischen Ausland und Übersee (z.B. Universität Udine, Universität Frankfurt, Universität Auckland (Neuseeland))

\section{Anerkennung der Leistungen des Fachbereichs}

Einige Mitglieder des Instituts waren in verschiedener Funktion als Gutachter und Herausgeber von Zeitschriften im In- und Ausland tätig. Eine Reihe wichtiger nationaler Preise ging an Wissenschaftler aus dem Institut.

\section{Institut für Mathematik B (Mathematische Optimierung, dynamische Systeme und Diskrete Ma- thematik)}

Raum-, Sach-, Personalausstattung

Das Institut verfügt über die ,übliche“ Ausstattung mit Büros, Seminarräumen, Extra-Räume für Start-Projekt und SFB, Bibliothek sowie Workstations. Es gibt pro MitarbeiterIn (incl. ProjektmitarbeiterIn) einen PC, 2 davon mit bis zu 1 GB RAM, 2 mit 1 GB RAM hochgerüstete PCs (zentral), 1 Dual-Prozessor-Workstation, 1 Alpha-Workstation.

Das Institut verfügt über folgende Finanzmittel 


\begin{tabular}{|l|r|r|r|}
\hline in $€$ & 2001 & 2002 & 2003 \\
\hline Laufende Ausgaben & 13193,85 & 9890,91 & 7096,87 \\
\hline Investitionen (Bundesmittel) & 8755,34 & 11572,45 & 3715,40 \\
\hline Drittmittel FWF (SFB) * & 180000,00 & 180000,00 & 180000,00 \\
Drittmittel (START) * & 150000,00 & 75000,00 & \\
\hline
\end{tabular}

*geschätzt

Das Institut beurteilt seine personelle Ausstattung anhand der finanziellen Möglichkeiten als angemessen. Es verfügt über 7 Stellen (2 Professuren, 4 wiss. Personal, 1 nichtwiss. Personal), die über Haushaltsmittel finanziert werden und 4 wiss. Mitarbeiter drittmittelfinanziert. Probleme gab es bei der Besetzung der vorhandenen wissenschaftlichen Stellen. Durch 3 Wegberufungen auf Professorenstellen sowie die mehrfache Beurlaubungen ergaben sich einige Besetzungslücken bzw. Engpässe im Bereich des Gebietes der mathematischen Optimierung (Nachbesetzungen erfolgten großteils auf anderen Gebieten, weil es keine qualifizierten Bewerber auf dem Gebiet der Optimierung zum richtigen Zeitpunkt gab).

Für das Institut ist die Bibliothek unabdingbar für die Forschung. Sinkendes Bibliotheksbudget bei ständig steigenden Preisen und stark eingeschränkter Online-Zugriff auf Literatur (besonders spürbare Einschnitte 2004 - u.a. Elsevier-Problem) beeinträchtigen die Forschung. Des weiteren werden Probleme mit Arbeitsplätzen für Drittmittelangestellte in den nächsten Jahren (bisher benutzte Räume in einem anderen Mietgebäude fallen ersatzlos weg) erwartet. Ein weiteres Defizit sieht das Institut im geringen Reisebudget.

\section{Besondere Aktivitäten in der Lehre}

Die Vorstellungen des Institut einer Doktorandenförderung sind: Promotion auf Drittmittelprojekt-Stellen oder eigenen Promotionsstellen (mit geringer Lehrbelastung), Auslandserfahrung sammeln durch Post Doc-Aufenthalt(e)/Auslandsstipendien, Förderung der Teilnahme an Workshops, Sommerschulen und ähnlichen wissenschaftlichen Veranstaltungen für junge Wissenschaftler am Beginn der Karriere.

\section{Publikationen}

Im Zeitraum 2001-2003 wurden von Mitgliedern des Instituts 61 wiss. Arbeiten veröffentlicht, 58 in referierten Zeitschriften, 3 Monographien. Im gleichen Zeitraum wurden 84 Vorträge gehalten, 47 davon auf Einladung. 


\section{Wissenschaftlicher Nachwuchs}

Im Zeitraum 1999 - 2003 gab es am Institut 4 Promotion mit einer durchschnittlichen Dauer von 2,83 Jahren. Das Durchschnittsalter lag bei 30 Jahren. Im gleichen Zeitraum gab es 3 Habilitationen, die mit einer durchschnittlichen Habilitationsdauer von 4,66 Jahren. Das Durchschnittalter lag bei 30 Jahren.

Doktoranden ohne Anstellung zur TU werden als Tutoren im Lehrbetrieb eingesetzt, in Einzelfällen als bezahlte Lehraufträge, wenn Geld vorhanden ist.

Habilitanden werden mit eigenen Lehrveranstaltungen bei Anstellung an der TU beauftragt, über Lehraufträge in externen Fällen.

Beim Besuch von Didaktikveranstaltungen geschieht die persönliche Betreuung und Beratung am Institut durch erfahrenere Lehrende.

Als Graduiertenstudium bietet das Institut ein Doktoratsstudium der techn. Wissenschaften an mit Spezialvorlesungen und Spezialseminare für Doktoranden (vielfach von ausländischen Gastwissenschaftlern abgehalten).

\section{Personalfluktuation}

In den letzten 5 Jahren haben 16 Wissenschaftler das Institut verlassen. In diesem Zeitraum gab es 5 Neuzugänge im Mittelbau. Schwierigkeiten bei der Personalfindung sieht das Institut im Pensionsrecht, welches die Berufungen aus dem Ausland erschwert.

Massive Schwierigkeiten qualifizierte Mitarbeiter für Vertretungsstellen mit kurzer Laufzeit und kurzer Anlaufzeit anzuwerben, sieht das Institut in der Verfügbarkeit der Stellen, die nur einige Monate vor Besetzung bekannt werden. Dieses wird durch das neue Dienstrecht, welches das Doktorat als Einstellungsvoraussetzung vorsieht, nach Meinung des Instituts noch verschärft.

Die deutlich geringere Bezahlung im Vergleich zur Industrie ist eine weitere Hemmschwelle für junge Mitarbeiter in Bereichen mit guten Berufschancen in der Industrie.

\section{Kooperation in der Forschung}

Das Institut unterhält Kooperationen mit Partnern in Kosice, Hradec Kralove, Prag, Krakau, Budapest und Laibach im Rahmen des Netzwerkes SK-47 CEEPUS. Es bestehen darüber hinaus zahlreiche Kooperationen mit verschiedenen Universitäten im Ausland (z.B. City University of Hong Kong, Aarhus University, Warwick Business School). Des weiteren bestehen vielfältige Kooperationen mit Industrieunternehmen. 
Anerkennung der Leistungen des Fachbereichs

Einige Mitglieder des Instituts waren in verschiedener Funktion als Gutachter und Herausgeber von Zeitschriften im In- und Ausland tätig. Eine Reihe wichtiger nationaler und internationaler Preise ging an Wissenschaftler aus dem Institut.

\section{Institut für Mathematik C (Mathematische Strukturtheorie)}

Raum-, Sach-, Personalausstattung

Das Institut verfügt über 9 Räume auf einer Gesamtnutzfläche von $170,80 \mathrm{~m}^{2}$ und gemeinsam mit dem Institut für Mathematik D über einen Seminarraum von 48,50 $\mathrm{m}^{2}$. An Nutzfläche für die Forschung sind 146,45 $\mathrm{m}^{2}$ vorhanden sowie 1 Fachbibliothek.

An technischer Ausstattung stehen zur Verfügung: 3 Notebooks, 12 PCs, 7 Drucker, 1 Farbdrucker, 1 Workstation sowie folgende Finanzmittel:

\begin{tabular}{|l|r|r|r|}
\hline in $€$ & 2001 & 2002 & 2003 \\
\hline laufende Ausgaben & $7.948,55$ & $7.785,20$ & $7.357,63$ \\
\hline Investitionen (Bundesmittel) & $* 7.252,75$ & $* 6.777,60$ & $4.665,60$ \\
\hline Investitionen (Drittmittel) & $1.901,63$ & & $6.620,00$ \\
\hline Einnahmen Bundesmittel & $7.994,01$ & $7.860,00$ & $96.188,10$ \\
\hline Drittmittel FWF & $21.489,72$ & $63.472,97$ & \\
\hline \multicolumn{2}{r|}{ Sonstige Drittmittel, } \\
laufen, aber dem Institut zuzuordnen sind \\
wie CD-Labors, Kompetenzzentren (bitte u. erläu- \\
tern)
\end{tabular}

* Inkl. Rest Berufungszusage ** Europäische Union - „Marie-Curie-Fellowship“

Die personelle Ausstattung wird vom Institut als zufriedenstellend betrachtet. Es bestehen 6,5 Stellen (1 Professur, 4,5 wiss.Personal (1,5 auf Zeit), 1 nichtwiss. Personal), die aus Haushaltsmitteln finanziert sind und 3 Stellen drittmittelfinanziert.

Bei der infrastrukturellen Ausstattung besteht nach Meinung des Instituts ein eklatanter Mangel an großen Hörsälen für Servicevorlesungen. Die Anzahl der Seminarräume ist nicht ausreichend. Des weiteren gibt es weder Rechnerräume für das Institut noch Aufenthaltsräume für die Studierenden. 


\section{Besondere Aktivitäten in der Lehre}

Es gibt gemeinsame Vorstellungen über die Promotionsdauer: ca. 3 Jahre; Habilitationsdauer nach Qualität und Anzahl (Richtwert 10) der referierten Publikationen; Aufgaben von Doktoranden/Habilitanden mit Dienstverhältnis am Institut: Mitarbeit in Lehre und Verwaltung in einem Ausmaß, das die Forschungsarbeit nicht beeinträchtigt; Maßnahmen zur Qualitätssicherung und Förderinstrumente: Internationale Kontakte, Tagungsteilnahmen, Auslandsaufenthalte im Rahmen von Post Doc- Grants, usw.

\section{Publikationen}

Im Zeitraum 2001-2003 hat das Institut folgende Publikationsleistungen erbracht:

42 referierte Aufsätze, 61 Vorträge davon 29 auf Einladung.

\section{Wissenschaftlicher Nachwuchs}

In den Jahren 1999-2002 gab es 4 Promotionen, die in einem Zeitraum von 5 Jahren abgelegt wurden. Das Durchschnittsalter lag bei 35 Jahren. In diesem Zeitraum gab es 1 Habilitation, die in 8 Jahren fertiggestellt wurde. Der Habilitand war 38 Jahre.

Dissertanten werden auf Assistentenstellen für Tutorien im Übungsbereich und im Rahmen von Lehraufträgen für die Organisation und Betreuung der Übungen zu Diskreter Mathematik für Telematik und Softwareentwicklung und Wissensmanagement eingestellt. Die finanziellen Einschränkungen machen es in Zukunft jedoch nach Meinung des Instituts schwer bis unmöglich, Projektmitarbeiter im Rahmen von Lehraufträgen in die Lehre einzubinden.

\section{Personalfluktuation}

In den letzten 5 Jahren haben 6 Wissenschaftler das Institut verlassen. Im gleichen Zeitraum sind 12 Wissenschaftler im Mittelbau in das Institut gekommen.

Grundsätzlich wurden Berufungen durch die wiederholten Dienstrechtsänderungen der letzten Jahre erschwert, die mit finanziellen Verschlechterungen einhergingen. Konkret standen aber am Institut für Mathematik C seit 1999 keine Neuberufungen an.

Alle Planstellen sind mit habilitierten, fest angestellten älteren Mitarbeitern besetzt, so dass es nur im Rahmen von Pensionierungen möglich sein wird, jüngeren qualifizierten Mitarbeitern am Institut eine längerfristige Perspektive zu eröffnen.

\section{Kooperation in der Forschung}

Das Institut unterhält Kooperationsbeziehungen mit verschiedenen Universitäten im In- und Ausland (z.B. Universität Leuven, Pennsylvania State University) und mit Industrieunterneh- 
men (Siemens, rmDATA). Über EU-Programme wie ERASMUS und Sokrates bestehen Austauschprogramme für Studenten.

\section{Anerkennung der Leistungen des Fachbereichs}

Einige Mitglieder des Instituts waren in verschiedener Funktion als Gutachter und Herausgeber von Zeitschriften im In- und Ausland tätig. Der Studienpreis der ÖMG für die beste Dissertation 2003 ging an einen Mitarbeiter des Instituts.

\section{Institut für Mathematik D (Numerik und Partielle Differentialgleichungen}

\section{Raum-, Sach-, Personalausstattung}

Das Institut verfügt über 6 Räume auf einer Gesamtnutzfläche von 143,80 m² und 1⁄2 Seminarraum à $24,25 \mathrm{~m}^{2}$ sowie über folgende Finanzmittel:

\begin{tabular}{|l|r|r|r|}
\hline \multicolumn{1}{|c|}{ in $€$} & 2001 & 2002 & 2003 \\
\hline laufende Ausgaben & 8.216 & 8.530 & 4.310 \\
\hline Investitionen (Bundesmittel) & 11.170 & 305 & -- \\
\hline Einnahmen Bundesmittel & 8.212 & 8.000 & 6.450 \\
\hline Drittmittel FWF & 48.000 & 48.000 & 24.000 \\
\hline
\end{tabular}

Nach Ansicht des Institutes bedarf die Personalausstattung eines Neuaufbaus, der bereits in Angriff genommen wird. Das Institut verfügt derzeit über 5,5 Stellen (davon 2 Professuren Em. und 1 i.R., 1 Mittelbau, 1,5 nichtwiss. Personal).

Nach der Emeritierung eines Lehrstuhlinhabers im Jahr 2002 haben die Neuausschreibung der Professur, die Arbeit der Berufungskommission, sowie vor allem die Berufungsverhandlungen längere Zeit in Anspruch genommen. Am 1.10.2004 wird diese Professur wieder besetzt sein. Das plötzliche Ableben des 2.Lehrstuhlinhabers hat eine weitere Lücke in den Personalstand gerissen, der nun einer entsprechenden Aufbauarbeit bedarf.

\section{Publikationen}

Das Institut hat in den letzten 3 Jahren 40 Publikationen veröffentlicht, darunter 24 referierte. Es wurden 32 Vorträge gehalten, 7 davon auf Einladung. 


\section{Wissenschaftlicher Nachwuchs}

In den letzten 5 Jahren gab es 3 Promotionen, mit einer Durchschnittsdauer von 3,5 Jahren.

Die Doktoranden waren durchschnittlich 30 Jahre alt. Im gleichen Zeitraum gab es keine Habilitationen.

\section{Personalfluktuation}

In den letzten 5 Jahren gab es 5 Abgänge von Wissenschaftlern und keine Neuzugänge. Neuberufungen sind durch die mehrfachen Dienstrechtsänderungen der letzten Jahre und die damit verbundene finanzielle Knappheit schwieriger geworden.

\section{Kooperation in der Forschung}

Es gibt Kooperationsbeziehungen zu, EPFL, Department of Analysis and Scientific Computing im Rahmen des EU-Projektes Partnerschaft RTN HaeMOdel. Des weiteren besteht eine Zusammenarbeit mit Universitäten im Ausland (Duke University, USA, ETH Zürich). Besondere Arbeitsbeziehungen bestehen zur Hanoi University of Technology.

Die Partnerschaft zum EU-RTN-Projekt HaeMOdel , „Mathematical Modeling of the Cardiovascular System“, wird in einem Sonderstatus durch einen Professor im Ruhestand weitergeführt.

\section{Anerkennung der Leistungen des Fachbereichs}

Von Mitgliedern des Institut wurden Gutachtertätigkeiten im Schweizer Nationalfond wahrgenommen sowie bei Fachzeitschriften.

\section{Institut für Geometrie}

\section{Raum-, Sach-, Personalausstattung}

Das Institut verfügt über eine Gesamtnutzfläche von $260,32 \mathrm{~m}^{2}$. Es verfügt über 10 PCs, 3 Laptops) und 2 Beamer, 1 Fachbibliothek und über folgende Finanzmittel:

\begin{tabular}{|l|r|r|r|}
\hline In $€$ & 2001 & 2002 & 2003 \\
\hline Laufende Ausgaben & k.A. & k.A. & k.A. \\
\hline Investitionen (Bundesmittel) & 10.000 & 10.000 & 14.500 \\
\hline Einnahmen Bundesmittel & -- & 7.500 & 5.000 \\
\hline $\begin{array}{l}\text { Sonstige Drittmittel, die organisatorisch nicht über Uni } \\
\text { laufen, aber dem Institut zuzuordnen sind wie CD- } \\
\text { Labors, Kompetenzzentren }\end{array}$ & 1.800 & $* 1.860$ & -- \\
\hline
\end{tabular}

(*) Tagungsunterstützung von Stadt Graz und Land Stmk 
Das Institut verfügt über 15 1/3 Stellen (2 Professuren, davon 1 vakant), 9 1/3 Mittelbau (1 1/3 auf Zeit), 2 Lehrbeauftragte und 2 nichtwiss. Personal), die aus Haushaltsmitteln bezahlt werden. Die Besetzung der vakanten Stelle erfolgt aus strategischen Gründen zeitversetzt. Diese personelle Lücke wird durch einen Gastprofessor teilweise aufgefangen. Das Institut kritisiert, dass dadurch die ohnehin schon überlasteten Mitarbeiter noch mehr belastet werden. Die Ausstattung der Bibliothek wird vom Institut bemängelt. Es gibt Probleme beim Zeitschriftenkauf und beim Zustand der Bibliotheksschränke. Des weiteren wünscht sich das Institut neue PCs.

\section{Publikationen}

Im Zeitraum 2001-2003 hat das Institut 17 Publikationen veröffentlicht, 9 davon in referierten Zeitschriften. Im gleichen Zeitraum wurden 29 Vorträge gehalten, 8 davon auf Einladung.

\section{Wissenschaftlicher Nachwuchs}

In den letzten 5 Jahren haben am Institut 4 Promotionen stattgefunden, die in einer durchschnittlichen Dauer von 3 Jahren geschrieben wurden. Das Durchschnittsalter lag bei 29 Jahren. Im gleichen Zeitraum gab es 1 Habilitation, die in 8 Jahren geschrieben wurde. Der Habilitand war 40 Jahre alt. Doktoranden und Habilitanden werden im gesetzlich vorgesehenen Ausmaß in die Lehre miteinbezogen, was wegen der hohen Lehrbelastung bis zur Höchstgrenze ausgeschöpft wird.

\section{Personalfluktuation}

In den letzten 5 Jahren sind 2 Wissenschaftler aus dem Institut ausgeschieden. Einer von ihnen aus Altersgründen. Im gleichen Zeitraum gab es 2 Neuzugänge im Mittelbau. Das Institut hat Schwierigkeiten bei der Nachbesetzung einer Universitätsprofessur. Es war bisher keine Ersatzkraft zu bekommen.

Ein hoher Prozentsatz der AbsolventInnen des Lehramtsstudiums im Unterrichtsfach Darstellende Geometrie hat im Berichtszeitraum AssistentInnenstellen an anderen österreichischen Universitäten erhalten: Zwei an der Universität Innsbruck, zwei an der TU Wien und einer an der Universität Linz. Die Qualität der Ausbildung in Graz wird offensichtlich sehr geschätzt.

\section{Kooperation in der Forschung}

Das Institut unterhält Kooperationsbeziehungen zu anderen Universitäten im europäischen Ausland und in Kanada. (z.B. TU Budapest, McGill University Montreal).

Die WissenschaftlerInnen des Institutes waren weit über das durchschnittliche Maß hinaus in die akademische Selbstverwaltung eingebunden: im gesamten Erhebungszeitraum als Dekan 
der TN- Fakultät der TU Graz und als Mitglied des Zentralausschusses der Hochschullehrer Österreichs sowie der PROKO sowie von Oktober 2000 bis Juli 2002 als Vorsitzender des Arbeitskreises für Gleichbehandlungsfragen der TU Graz.

\section{Anerkennung der Leistungen des Fachbereichs}

Viele Mitglieder des Instituts waren in verschiedener Funktion als Gutachter und Herausgeber von Zeitschriften im In- und Ausland tätig

\section{Institut für Statistik}

\section{Raum-, Sach-, Personalausstattung}

Die infrastrukturelle Ausstattung besteht aus: 10 Räumen (6 Büros, 1 Sekretariat, 1 Seminarraum, 1 Computerlabor, 1 Bibliothek) auf einer Nutzfläche von $266 \mathrm{~m}^{2}$. An Geräten stehen zur Verfügung: 13 PCs mit Vernetzung, 2 Scanner, 2 Laptops, 6 Drucker, 1 Beamer. Folgende Finanzmittel stehen zur Verfügung:

\begin{tabular}{|l|r|r|r|}
\hline in $€$ & 2001 & 2002 & 2003 \\
\hline laufende Ausgaben & $8.430,05$ & $5.500,00$ & $4.000,00$ \\
\hline Investitionen (Bundesmittel) & $1.250,000$ & $6.700,00$ & $2.912,40$ \\
Investitionen (Drittmittel) & 0,00 & $2.101,76$ & 0,00 \\
& & & \\
\hline Einnahmen Bundesmittel & $8.430,05$ & $5.500,00$ & $4.000,00$ \\
\hline Sonstige Drittmittel & 563,62 & $17.356,50$ & $39.382,49$ \\
\hline
\end{tabular}

Am Institut gibt es 6 Stellen (2 Professuren, 4 wiss. Personal (davon 2 auf Zeit)), die aus Haushaltsmitteln bezahlt werden.

Die Personalausstattung wird vom Institut als angemessen angesehen.

Das Institut sieht als Defizit die Minderung der Einnahmen von $8.400 €$ auf $4.000 €$ für die laufenden Ausgaben. Nach Ansicht des Institutes wären Einnahmen von 6.000€ angemessen.

\section{Publikationen}

Im Zeitraum 2001-2003 sind am Institut 27 Publikationen erschienen, davon 17 als referierte Aufsätze. Es wurden insgesamt 19 Vorträge gehalten, davon 8 auf Einladung. 


\section{Wissenschaftlicher Nachwuchs}

In den letzten 5 Jahren wurden 3 Promotionen mit einer durchschnittlichen Promotionsdauer von 3,6 Jahren abgelegt. Das Durchschnittsalter der AbsolventInnen lag bei 31,5 Jahren. Habilitationen gab es in diesem Zeitraum keine.

Die Doktoranden und Habilitanden werden durch folgenden Maßnahmen auf die Lehre vorbereitet:

- Betreuung von Übungen zusammen mit Tutoren

- Abhaltung kleiner Vorlesungseinheiten im Ausmaß von max. 4 Semesterwochenstunden im Jahr

- Ausarbeitung und Abhaltung von Präsentationen und Seminarvorträgen

- Regelmäßiger Erfahrungsaustausch mit Professoren

\section{Personalfluktuation}

Durch die Emeritierung des Lehrstuhlinhabers und das gleichzeitige Ausscheiden eines promovierten Univ.-Assistenten am 30.9.2001 ergab sich eine verstärkte Belastung des verbleibenden Personals in der Lehre und Administration. Die Professur konnte am 1.3.2003 wieder besetzt werden. Die Assistentenstelle wurde geteilt und durch 2 wissenschaftliche Mitarbeiter (1/2-Stellen) am 1.10.2001 bzw. 1.8.2003 nachbesetzt.

\section{Kooperation in der Forschung}

Das Institut unterhält Kooperationen mit Hochschulen in europäischen In- und Ausland und den USA (z.B. TU Darmstadt, University of Ljubljana, Utah State University) und mit Industrieunternehmen und außeruniversitären Forschungseinrichtungen (z.B. AMS = Austria Micro Systems, Steewag-Steg). Des weiteren bestehen Kooperationen innerhalb von EU-

Netzwerken mit den Umweltämtern der Städte Klagenfurt und Graz.

Anerkennung der Leistungen des Fachbereichs

Einige Institutsmitglieder waren in verschiedenen Funktionen als Gutachter für Fachzeitschriften im In- und Ausland tätig. Ein Mitglied war in verschiedenen Beiräten tätig. Eine Reihe wichtiger internationaler Ehrungen gingen an ein Mitglied des Instituts. 


\section{Teil B: Lehre und Studium am Fachbereichs Mathematik an der TU Graz}

\section{Ausbildungsprofil}

Die Mathematik Institute der TU Graz bieten ein anwendungs- und forschungsorientiertes Diplom- und Doktoratsstudium der Technischen Mathematik mit folgenden 3 spezifischen Schwerpunkten an

Österreichweites Zentrum: Diskrete Mathematik (inklusive Theoretische Informatik), Optimierung, Zahlentheorie (gemeinsam mit KFU)

Regionales Zentrum: Stochastik, Finanz- und Versicherungsmathematik Angestrebtes regionales Zentrum: Geometrie, Technomathematik (durch Neuberufungen)

\section{Allgemeine Ziele}

Ziel des Diplomstudiums Technische Mathematik an der Technischen Universität Graz ist es, die AbsolventInnen vor allem in der industriellen Forschung und Entwicklung; der Erstellung und Umsetzung mathematischer Modelle in der Wirtschaft sowie im Finanzund Versicherungswesen; in der theoretischen und praktischen Behandlung von Problemstellungen aus dem Bereich der Informatik und in der universitären Forschung und Lehre auszubilden.

Ziel des Studiums ist es daher, die Studierenden mit den wissenschaftlichen Methoden der Technomathematik, des Operations Research, der Statistik, Finanzmathematik, Versicherungsmathematik und Informationsverarbeitung sowie deren Entwicklung und Anwendung vertraut zu machen, wobei der Vielfalt der Methoden und der Pluralität der Theorien Rechnung getragen wird. Insbesondere sollen die Studierenden befähigt werden, in ihrem zukünftigen beruflichen Umfeld die Theorien und Methoden auf reale Problemstellungen anwenden zu können.

Diese Studienziele sind im Studienplan, der mit 01.10.2000 in Kraft ist, formuliert. Ein neuer dreigliedriger Studienplan ist in Vorbereitung und soll mit 01.10.2005 in Kraft treten. Die Verständigung über Studienziele und Leistungsstandards fand bisher in der Studienkommission statt und wird ab jetzt in der Arbeitsgruppe Studienplan stattfinden.

Es liegt ein Entwurf für ein Bakkalaureats-/Magisterstudium vor, der im Laufe des Studienjahres 2004/05 der Curricularkommission vorgelegt werden und mit 1.10.2005 in Kraft treten soll. 


\section{Praxisbezug}

Die AbsolventInnen sind nach Abschluss des Studiums vor allem in folgenden Bereichen tätig:

- industrielle Forschungs- und Entwicklungseinrichtungen;

- Planungseinrichtungen der Industrie und des öffentlichen Dienstes;

- Finanzdienstleistungsunternehmen(Versicherungen, Banken, usw.) und in Unternehmensberatungsgesellschaften; in der Informationstechnologie

- Handelsunternehmen (Logistik);

an Universitäten, Fachhochschulen und in außeruniversitären Forschungsinstituten.

An der Konzeption der Studienpläne waren Vertreter der lokalen Wirtschaft beteiligt. Darüber hinaus bestehen Kontakte zur lokalen Versicherungswirtschaft, Industrie und zu Kompetenzzentren, die ebenfalls Einfluss auf die Gestaltung der Studienpläne haben. Diese Kontakte ermöglichen die Abfassung von praxisorientierten Diplomarbeiten und Dissertationen im Zusammenarbeit mit Firmen. Es gibt Lehrveranstaltungen von Praktikern.

Der Bedarf an praxisorientierten Lehrveranstaltungen wurde artikuliert und im neuen Studienplan berücksichtigt.

\section{Personelle Ressourcen}

Die Lehrkapazität des festen Lehrkörpers (10 Professoren, 16 Dozenten, 10,5 Mittelbau) sollte 436 SWS pro Studienjahr betragen. Die tatsächlich erbrachten Lehrleistungen nach Grundund Hauptstudium der letzten 5 Jahre lag zwischen 390 SWS in 1990 und 419 SWS in 2003. An Serviceleistungen für andere Studienbereiche wurden 139 SWS erbracht. Der feste Lehrkörper besteht derzeit aus 9 Professoren, 15 Dozenten und 6 Mittelbauangehörigen. Weitere 4,5 Mittelbaudienstposten sind Dissertationsstellen, die in der Lehre nicht entsprechend herangezogen werden können. Die tatsächlich erbrachten Lehrleistungen im Grund- und Hauptstudium (Technische Mathematik und Lehramt Darstellende Geometrie) in den letzten 5 Jahren betrug im Durchschnitt 400 SWS. Dazu kommen im Durchschnitt 140 SWS Service für andere Studienrichtungen. Die durchschnittliche Lehrbelastung pro Semester beträgt daher 9 Wochenstunden pro Semester.

\section{Sachausstattung}

Es stehen 5 Laborarbeitsplätze bzw. Großgeräte für die Lehre zur Verfügung. Es gibt 9 PCLehrsäle des Zentralen Informatikdienstes mit jeweils ca. 30 Arbeitsplätzen. Die Bibliothek bietet eine Lehrbuchsammlung an, die regelmäßig den Wünschen der Lehrenden angepasst wird. Die Informationsdienste der Bibliothek stehen den Studierenden zur Verfügung. 
Der Fachbereich beurteilt die räumliche und sachliche Ausstattung für die Lehre als ausreichend. Es bestehen keine Defizite.

Durch die Implementierung des UG 2002 (UG = Universitätsgesetz) mussten finanzielle Einsparungsziele bei der Betreuung erreicht werden $(-16 \%)$.

Studienanfänger

\begin{tabular}{|l|r|r|r|r|r|}
\hline TM & 1999 & 2000 & 2001 & 2002 & 2003 \\
\hline männlich & 28 & 36 & 27 & 37 & 24 \\
\hline weiblich & 12 & 26 & 20 & 24 & 22 \\
\hline zusammen & $\mathbf{4 0}$ & $\mathbf{6 2}$ & $\mathbf{4 7}$ & $\mathbf{6 1}$ & $\mathbf{4 6}$ \\
\hline
\end{tabular}

TM=Technomathematik

\begin{tabular}{|l|r|r|r|r|r|}
\hline LA DG & 1999 & 2000 & 2001 & 2002 & 2003 \\
\hline männlich & 0 & 2 & 0 & 2 & 2 \\
\hline weiblich & 1 & 1 & 0 & 1 & 0 \\
\hline zusammen & $\mathbf{1}$ & $\mathbf{3}$ & $\mathbf{0}$ & $\mathbf{3}$ & $\mathbf{2}$ \\
\hline
\end{tabular}

LA=Lehramt

In Relation beträgt die Zahl der Studienanfänger

je Professorensteile

\begin{tabular}{|l|l|l|l|l|l|}
\hline TM & 1999 & 2000 & 2001 & 2002 & 2003 \\
\hline & 4 & 6,2 & 4,7 & 6,1 & 4,6 \\
\hline
\end{tabular}

je Stelle Wissenschaftliches Personal

\begin{tabular}{|l|l|l|l|l|l|}
\hline TM & 1999 & 2000 & 2001 & 2002 & 2003 \\
\hline & 1,1 & 1,7 & 1,3 & 1,8 & 1,3 \\
\hline
\end{tabular}

Studien- und Prüfungsordnung

Laut Studienplan gibt es eine empfohlene Reihenfolge des Besuchs der Lehrveranstaltungen.

Im Regelfall gibt es zu jeder Pflichtveranstaltung eine zugehörige Übungseinheit.

\begin{tabular}{|l|l|l|l|l|}
\hline Fächer & TM St.zwg: A & TM St.zwg: B & TM St.zwg: C & LA DG \\
\hline Pflichtfächer & 124 & 120 & 120 & 72 \\
\hline Wahlfächer & 21 & 25 & 25 & 10 \\
\hline Freie Wahlfächer & 16 & 16 & 16 & 10 \\
\hline
\end{tabular}

St.zwg = Studienzweig 
Die Zuordnung von Lehrveranstaltungen zu Prüfungsfächern ist sowohl im Studienplan als auch im Informationssystem TUG Online ersichtlich. Die Verteilung der Lehraufgaben erfolgt einvernehmlich in einer allgemeinen Diskussion. Die Einführungslehrveranstaltungen werden daher von Professoren und habilitiertem Mittelbau wechselweise abgehalten.

Die Koordination des Lehrangebotes wird vom Studiendekan vorgenommen.

Die Pflichtfächer werden in jeden Studienjahr angeboten. Der Studienplan bietet im Bereich der Wahlfächer sehr große Freiheiten. Die wissenschaftlich am stärksten vertretenen Wahlfächer werden jährlich angeboten.

An Auslandsstudienprogrammen gibt es ERASMUS Lehrendenmobilität/ERASMUS Studentenaustausch. Im Studienjahr 2003/04 nahmen 10 Studierende der Technischen Mathematik an Auslandsstudienprogrammen teil.

\section{Prüfungsverfahren}

Es werden mindestens 3 Prüfungstermine pro Lehrveranstaltung und Semester angeboten.

Die Studierenden suchen ihre Prüfer selbst aus. Bei Diplomprüfungen wird die Auswahl der Prüfer durch den Studiendekan bestätigt.

Für die Abfassung der Diplomarbeit sieht der Studienplan 1 Semester (30 ECTS) vor. Die Diplomarbeiten haben einen Umfang von ca. 80 Seiten. Tatsächlich werden für die Abfassung der Diplomarbeiten ein bis zwei Semester benötigt. Es werden pro Semester mindestens 3 feste Prüfungstermine angeboten. Die Korrektur der Prüfungsarbeiten erfolgt üblicherweise innerhalb einer Woche. Laut Satzung der TU Graz kann jede Prüfung dreimal wiederholt werden.

\section{Leistungsanforderungen}

Vorgegeben sind im Mittel 20 Semesterwochenstunden pro Semester. Der Studienplan ist daher nach Meinung der Fakultät in der Planstudienzeit studierbar.

In den einzelnen Studiengängen müssen folgende Leistungsnachweise erbracht werden:

\begin{tabular}{|l|r|r|r|r|}
\hline & TM St.zwg. A & TM St.zwg B & TM St.zwg. C & LA DG \\
\hline Prüfungsakte & 70 & 63 & 68 & 33 \\
\hline
\end{tabular}




\section{Studienerfolg}

Zahl der abgelegten Zwischenprüfungen/Vordiplome der letzten 5 Prüfungsjahre (WS und darauf folgendes SS) nach Studiengängen/Abschlüssen

\begin{tabular}{|l|r|r|r|r|r|}
\hline Studiengang & \multicolumn{6}{|c|}{ 1. Diplomprüfung (von 2 Abschnitten; alter Studienplan-4 Semester) } \\
\hline TM & 1999 & 2000 & 2001 & 2002 & 2003 \\
\hline & 25 & 20 & 13 & 10 & 2 \\
\hline
\end{tabular}

\begin{tabular}{|l|r|r|r|r|r|}
\hline Studiengang & \multicolumn{5}{|c|}{ 1. Diplomprüfung (von 3Abschnitten; neuer Studienplan-2Semester) } \\
\hline TM & 1999 & 2000 & 2001 & 2002 & 2003 \\
\hline & 0 & 1 & 15 & 28 & 11 \\
\hline
\end{tabular}

\begin{tabular}{|l|r|r|r|r|r|}
\hline Studiengang & \multicolumn{6}{|l|}{ 1. Diplomprüfung - 4 Semester } \\
\hline LA DG & 1999 & 2000 & 2001 & 2002 & 2003 \\
\hline & 5 & 3 & 3 & 5 & 1 \\
\hline
\end{tabular}

\begin{tabular}{|l|r|r|r|r|r|}
\hline Studiengang & \multicolumn{6}{|c|}{ 2. Diplomprüfung (von 3 Abschnitten; neuer Studienplan-4 Semester) } \\
\hline TM & 1999 & 2000 & 2001 & 2002 & 2003 \\
\hline & 0 & 0 & 0 & 0 & 10 \\
\hline
\end{tabular}

Zahl der Absolventen/innen der letzten 5 Prüfungsjahre (WS und darauf folgendes SS) nach Studiengängen/Abschlüssen

\begin{tabular}{|l|r|r|r|r|r|}
\hline Studiengang & 1999 & 2000 & 2001 & 2002 & 2003 \\
\hline TM & 22 & 24 & 16 & 17 & 30 \\
LA DG & & & & 6 & 2 \\
\hline
\end{tabular}




\section{Qualitätssicherung}

Die Lehrveranstaltungen des ersten Studienjahres werden jährlich verpflichtend von den Studierenden evaluiert. Die Ergebnisse sind im Informationssystem TUG Online zugänglich. Die Verbesserung von Ausbildung und Lehrleistungen werden regelmäßig in der Studienkommission beraten. Die Universität hat dazu auch eine neue Arbeitsgruppe Studienplan eingesetzt.

Defizite bei der Vorbildung der Studienanfänger werden im gravierenden Niveauunterschied der Studienanfänger im mathematisch-naturwissenschaftlichen Bereich gesehen. Diesem wird durch das Angebot von vorbereitenden Lehrveranstaltungen wie etwa Mathematik 0 und Programmieren 0 entgegengewirkt.

Die Universität plant die Einführung einer Join In- Prüfung als Anreiz. Von dem geplanten Bakkalaureatstudium wird eine Verkürzung der Studienzeit erwartet.

Die didaktische Qualifikation stellt einen wesentlichen Schritt im Habilitationsverfahren dar. Die studentischen Mitglieder im Habilitations- und Berufungskommissionen legen besonderes Augenmerk auf die didaktische Qualifikation.

Die Stärken werden in der hohen Aktualität in der Lehre durch die vorhandene Forschungskompetenz gesehen. Ein Mangel ist die schwache Einbindung der Geometrie in das Studium der Technischen Mathematik. 


\subsection{Universität Graz}

\section{Teil A: Forschung, Wissenschaftlicher Nachwuchs}

\section{Institut für Mathematik und wissenschaftliches Rechnen}

\section{Folgende 4 Schwerpunkte des Instituts nehmen an der Evaluierung teil:}

- Algebra und Zahlentheorie

- - Kontrolltheorie, Optimierung und wissenschaftliches Rechnen

- Analysis

- Modellierung und dynamische Systeme

\section{Raum-, Sach-, Personalausstattung}

Das Institut verfügt über 29 Büros auf einer Gesamtnutzfläche von $540 \mathrm{~m}^{2}$, weitere 8 Büros für Postdocs, Doktoranden und Gäste $\left(160 \mathrm{~m}^{2}\right)$ sowie 3 Seminarräume für je max. 30 Studenten, 1 Seminarraum mit 10 PC-Arbeitsplätzen, 1 Hörsaal für 140 Studenten, 3 kleinere Räume für Server, Drucker und Workstations und 1 Bibliothek (370m²). Die räumliche Ausstattung wird vom Institut als ausreichend angesehen. Für den zukünftig einzurichtenden Forschungsschwerpunkt Computerarchitektur und Hochleistungsrechnen müsse Platz geschaffen werden.

Die Fachbibliothek für Mathematik sei die wichtigste infrastrukturelle Einheit im Institut. Sie verfügt über 350 Zeitschriften und ca. 35.000 Monographien. Wegen der stagnierenden finanziellen Situation kämpft das Institut darum, den Standard der Bibliothek zu halten. Seit einigen Jahren werden Zeitschriften zusammen mit der TU Graz angeschafft, was Doppelanschaffungen vermeidet.

Es gibt einen Seminarraum mit 10 Computerarbeitsplätzen. Dies deckt gerade noch die Bedarfe für eine ordnungsgemäße Durchführung des Diplom- und des Lehramtsstudiums ab. Der Zugriff auf den Zentralen Informations-Dienst ZID ist zusätzlich gegeben. Für die Forschungserfordernisse sowie die Abwicklung von Diplomarbeiten und Dissertationen sind-2 Workstations und 5 Hochleistungs-PCs vorhanden. Es fehlen ausreichende Computerlabore für die Studenten besonders mit Einrichtung des Curriculums Computational Sciences. Das Institut überlegt, die Studenten mit Laptops auszustatten, so dass normale Seminarräume als Computerlabore mitgenutzt werden können. 
Durch die Pläne des Instituts, einen Schwerpunkt im Wissenschaftlichen Rechnen zu setzen, ist die Ausstattung mit Infrastruktur für das Höchstleistungsrechnen aus Sicht des Instituts unabdingbar. Die Naturwissenschaftliche Fakultät hat eine Zuwendung von $€ 830.000$ erhalten, um die Computerausstattung im Bereich Hochleistungsrechnen zu verbessern

Die Universität Graz besitzt wie alle anderen österreichischen Universitäten seit dem 1. 1. 2004 keine Planstellen mehr. Personal wird seit diesem Datum ausschließlich über privatrechtliche Dienstverträge eingestellt. Zurzeit besteht das wissenschaftliche Personal überwiegend aus Beamten und Beamtinnen, die Planstellen des Ministeriums innehaben und an die Universität ausgeliehen sind. Scheidet eine dieser Personen aus dem aktiven Dienst aus, so erlischt die zugehörige Planstelle. Man kann daher nicht von vakanten Planstellen sprechen, wohl aber von fehlendem Personal. Die Möglichkeit, Personal einzustellen, ist allein durch die budgetäre Lage bestimmt.

Algebra und Zahlentheorie: 1 Professor, 2 Dozenten, 1 Assistent, 1 Bundeslehrer (halbtägig), 2 wiss.Mitarbeiter (drittmittelfinanziert), 1 nichtwiss. Mitarbeiter (halbe Stelle)

Analysis: 2 Professoren, 2 Dozenten, 1 wiss. Mitarbeiter (ohne Vertrag), 1 nichtwiss. Personal

Kontrolltheorie, Optimierung und Wissenschaftliches Rechnen: 2 Professoren, 6 Dozenten, 2 Assistenten, 1 wiss. Beamten, 7 wiss. Mitarbeiter (drittmittelfinanziert), 2 nichtwiss. Mitarbeiter

Modellierung und dynamische Systeme: 2 Professoren, 3 Dozenten, 1 Assistent, 3 wiss. Mitarbeiter (drittmittelfinanziert), 1 nichtwiss. Personal.

Es gibt 1 neu zugewiesene Professur, für die das Besetzungsverfahren derzeit läuft.

Das Institut beurteilt seine personelle Ausstattung für die aktuell zu leistende Lehrtätigkeit als gerade ausreichend. Sollte das Curriculum Computational Sciences eingerichtet werden, würde die Lehrkapazität an seine Grenzen stoßen. Die Lehrverpflichtungen für Hochleistungsrechnen und Computerwissenschaften können nach Ansicht des Instituts schon jetzt nicht 
ausreichend befriedigt werden. Das Institut sieht dringenden Bedarf an zusätzlichen Assistentenstellen.

Zusätzlicher dringender Stellenbedarf besteht ebenfalls in der Forschung, insbesondere in den Bereichen Hochleistungsrechnen und Modellierung in der Physiologie.

Das Institut als Ganzes hat folgende Finanzmittel zur Verfügung, die von der Universität bereitgestellt werden:

\begin{tabular}{|c|c|c|c|}
\hline Kostenkategorie $\quad$ in $€$ & 2001 & 2002 & 2003 \\
\hline Laufende Ausgaben & 16.406 & 14.270 & 10.348 \\
\hline Literatur & 11.720 & 22.621 & 9.003 \\
\hline Investitionen & 45.748 & 76.704 & 22.260 \\
\hline Summe & 73.874 & 113.595 & 41.611 \\
\hline
\end{tabular}

An eingeworbenen Drittmitteln stehen zur Verfügung:

\begin{tabular}{|l|l|r|r|r|}
\hline Forschungsschwerpunkt & & 2001 & 2002 & 2003 \\
\hline Algebra und Zahlentheorie & FWF & 24.674 & 13.365 & 6.505 \\
\hline Analysis & FWF & 28.946 & 28.946 & 3.405 \\
& andere & & & 3.125 \\
\hline Kontrolltheorie, Optimierung und & FWF & 91.172 & 116.305 & 178.570 \\
Wissenschaftliches Rechnen & andere & 5.814 & 2.250 & 750 \\
\hline Modellierung und dynamische & FWF & 120.555 & 105.065 & 83.378 \\
Systeme & andere & 2.134 & 771 & 901 \\
\hline Summe & & $\mathbf{2 7 3 . 2 9 5}$ & $\mathbf{2 6 6 . 7 0 2}$ & $\mathbf{2 7 6 . 6 3 4}$ \\
\hline
\end{tabular}

\section{Publikationen}

Das Institut hat insgesamt im Zeitraum 2001-2004 folgende Publikationsleistung erbracht: 501 Monographien, Publikationen in Zeitschriften- und Proceedingsbänden sowie 316 Vorträge. 
Davon entfielen auf die einzelnen Forschungsschwerpunkte:

\begin{tabular}{|l|r|r|}
\hline Forschungsschwerpunkt & Publikationen & Vorträge \\
\hline Algebra und Zahlentheorie & 76 & 52 \\
\hline Analysis & 65 & 68 \\
\hline $\begin{array}{l}\text { Kontrolltheorie, Optimierung und Wissen- } \\
\text { schaftliches Rechnen }\end{array}$ & 260 & 139 \\
\hline Modellierung und dynamische Systeme & 100 & 57 \\
\hline
\end{tabular}

\section{Wissenschaftlicher Nachwuchs}

Im Zeitraum 1999 bis Oktober 2004 wurden insgesamt 13 Doktoratsstudierende betreut (davon 10 mit Abschluss an der Universität Graz, 1 mit Abschluss in Ägypten, 2 im SandwichProgramm mit der UGM in Yogyakarta, Indonesien). Im gleichen Zeitraum gab es 5 Habilitationen, die in einem Zeitrahmen von 5 Jahren nach Abschluss des Promotionsstudiums geschrieben wurden. Das Durchschnittsalter der Kandidaten lag bei 37,4 Jahren-, wobei zu berücksichtigen ist, dass nicht alle Kandidaten die Arbeit an ihrer Habilitation unmittelbar nach der Promotion in Angriff nahmen.

In der überwiegenden Zahl der Fälle erhielten Doktoranden entweder ein Forschungsstipendium oder wurden in einem Projekt angestellt. Sie haben in beiden Fällen keine Lehrverpflichtungen. Habilitanden waren meist Assistenten am Institut und hatten als solche Lehrverpflichtungen.

\section{Personalfluktuation}

Im Zeitraum 1999-2003 verließen 8 Wissenschaftler das Institut, 1 davon aus Altersgründen. Im gleichen Zeitraum hat es 3 Neubesetzungen gegeben.

Das Institut sieht die Schwierigkeiten in der Neubesetzung von Stellen in der neuen gesetzlichen Situation. Eine Verbeamtung ist seit 1.1.2004 nicht mehr möglich, da die Universität seit 1.1.2004 ihre Mitarbeiter als Privatangestellte einstellt und die vollen Gehaltskosten hierfür deutlich höher sind als bisher. Dies hat naturgemäß negative-Auswirkungen auf das Gesamtbudget und die Stellenbesetzungen. 


\section{Kooperation in der Forschung}

Das Institut unterhält zahlreiche Kooperationsbeziehungen zu Universitäten und anderen Forschungseinrichtungen im In- und Ausland (nähere Einzelheiten siehe ausführliche Unterlagen).

Anerkennung der Leistungen des Instituts

Die Mitglieder des Instituts waren in verschiedener Funktion als Gutachter und Herausgeber von Zeitschriften oder als Mitglieder von wissenschaftlichen Beiräten tätig. Eine Reihe von nationalen und Internationalen Auszeichnungen gingen an Mitglieder des Instituts. 


\section{Teil B: Lehre und Studium am Institut für Mathematik und wissenschaftliches Rechnen der Technischen Universität Graz}

Ausgangslage

An der Universität Graz können folgende Abschlüsse gemacht werden:

- Diplomstudiengang mit 2 Schwerpunkten: Allgemeine Mathematik sowie

Numerische Mathematik und Modellierung

- Lehramt für Mathematik

- Doktoratsstudium

AbsolventInnen des Lehramtsstudiums und des Diplomstudienganges schließen mit dem Akademischen Grad Magister/Magistra der Naturwissenschaften ab. Die AbsolventInnen des Doktoratsstudiums erwerben den Abschluss: Doktor/Doktorin der Naturwissenschaften.

\section{Allgemeine Ziele}

Das Ziel der beiden Diplomstudiengangs-Zweige in Mathematik ist es, eine solide wissenschaftliche Ausbildung in Mathematik zu vermitteln, als Hintergrund für mathematisch orientierte Berufe in Industrie und Handel einerseits und für den Start einer wissenschaftlichen Karriere als Mathematiker andererseits.

Der Ausbildungszweig Allgemeinmathematik betont die breite Vermittlung der mathematischen Disziplinen kombiniert mit der Fähigkeit, Abstraktionen auf hohem Niveau durchzuführen.

Der Ausbildungszweig Numerische Mathematik und Modellierung legt den Schwerpunkt der Ausbildung auf Mathematik in verschiedenen angewandten Wissenschaften. Es setzt daher den Akzent auf mathematische Modellierung und numerische Mathematik. Darüber hinaus soll es die Absolventen befähigen, mit angewandten Wissenschaftlern im Team zusammenzuarbeiten.

Ziel des Lehramtsstudiums ist die Berufsausbildung zur Lehrerin/zum Lehrer an Höheren Schulen.

Ferner bereitet das Studium auf ein weiterführendes Doktoratsstudium vor. Das Studium schließt mit der Verleihung des Titels „Magistra/Magister der Naturwissenschaften ab.

Sowohl das Diplomstudium als auch das Lehramtsstudium gibt den StudentInnen die Möglichkeit, 10\% der für das Studium vorgesehenen Semesterstunden in Form eines „Freien 
Wahlfaches“ zu absolvieren. Die Lehrveranstaltungen des Freien Wahlfaches können ohne Einschränkung aus dem Lehrangebot österreichischer Universitäten gewählt werden. Die Absolvierung dieser Lehrveranstaltungen ist durch Prüfungen nachzuweisen.

\section{Praxisbezug}

Im Lehramtsstudiengang besteht der Praxisbezug in Seminaren bzw. Lehreinheiten zum Unterrichten von Mathematik an Gymnasien in Graz. Der Praxisbezug der Ausbildung der LehramtsstudentInnen wird von Dozenten übernommen, die Teilzeitstellen an Höheren Schulen innehaben.

Das Institut steht einem Studiengang Bakkalaureat/Magister Artium positiv gegenüber: Entsprechende Diskussionen in der Studienkommission laufen.

\section{Personelle Ressourcen}

Die gesetzlich vorgesehene Lehrkapazität des festen Lehrkörpers (7 Professoren, 13 Dozenten, 3 Assistenten, 0.5 Mittelschullehrer) umfasst 239 SWS. Die tatsächlich erbrachte Lehrleistung der Institutsmitglieder liegt bei 145,5 SWS-infolge der Beschränkungen des Budgets für die Lehre.

Die tatsächlich erbrachte Lehrleistung der Institutsmitglieder zeigt die folgende Aufstellung: Univ.-Professoren: 52 SWS

Dozenten : 76 SWS

Assistenten: 11,5 SWS

Mittelschullehrer: 6 SWS

Das Institut bietet 45 SWS Service-Lehrveranstaltungen für folgende Studienrichtungen an:

- Umwelt-System-Wissenschaften: 29 SWS

- Biologie: 12 SWS

- Erdwissenschaften: 4 SWS

Bei Einführung des Studienganges Computational Sciences kämen auf das Institut weitere 65 SWS an Lehrverpflichtungen hinzu. 


\section{Sachausstattung}

An räumlicher Ausstattung steht zur Verfügung: 1 Seminarraum mit 10 PCs. Workstations und Hochleistungsrechner für Diplom- und Doktorarbeiten. Die Bibliothek ist gut ausgestattet für die laufenden Lehraktivitäten.

\section{Zahl der Studierenden $(\mathrm{m} / \mathrm{w})$}

\begin{tabular}{|ll|r|r|r|}
\hline Fach & & 2002 & 2003 & 2004 \\
\hline Doktorat & österreichisch & $8 / 1$ & $4 / 0$ & $2 / 0$ \\
& ausländisch & $1 / 0$ & - & - \\
& österreichisch & $45 / 23$ & $51 / 29$ & $49 / 26$ \\
& ausländisch & $3 / 3$ & $3 / 6$ & $1 / 6$ \\
\hline Diplomstudiengang & österreichisch & $115 / 144$ & $118 / 142$ & $82 / 108$ \\
& ausländisch & $1 / 2$ & $1 / 2$ & $1 / 1$ \\
\hline Summe Studienanfänger & insgesamt & $\mathbf{3 4 6}$ & $\mathbf{3 5 6}$ & $\mathbf{2 7 6}$ \\
\hline
\end{tabular}

Die Zahl der Studienanfänger ist zwischen 2003 und 2004 um 22\% gesunken, was durch den Einbruch im Lehramtsstudiengang verursacht wurde.

Studierende $(\mathrm{m} / \mathrm{w})$ im 1. Semester ihres Fachstudiums

\begin{tabular}{|ll|r|r|r|}
\hline Fach & & 2002 & 2003 & 2004 \\
\hline Doktorat & österreichisch & 0 & 0 & 0 \\
& ausländisch & & & \\
\hline Diplomstudiengang & österreichisch & $14 / 6$ & $15 / 8$ & $8 / 3$ \\
& ausländisch & $1 / 0$ & $0 / 2$ & $1 / 1$ \\
\hline Lehramtsstudiengang & österreichisch & $17 / 29$ & $17 / 24$ & $21 / 18$ \\
& ausländisch & $0 / 1$ & - & $1 / 0$ \\
\hline Summe Studenten & insgesamt & 68 & 66 & 53 \\
\hline
\end{tabular}

Zwischen 2003 und 2004 ist ein Rückgang bei den Studierenden insgesamt von 19 \% zu verzeichnen. Bei den Studierenden des Diplomstudienganges sogar um $48 \%$.

\section{Studien- und Prüfungsordnung}

Das Doktoratsstudium umfasst 4 Semester. Neben der Doktorarbeit muss der Student Kurse im Umfang von 16 SWS absolvieren. Davon kann er 4 SWS aus Fächern, die seine Doktorarbeit betreffen, frei wählen. 
Das Diplomstudium umfasst 120 SWS und ist auf eine Regelstudienzeit von 10 Semestern angelegt. Es gibt 3 Studienabschnitte:

- $\quad$ 1.Studienabschnitt (2 Semester mit 25 SWS) für die beiden Zweige Allgemeine Mathematik und Numerische Mathematik

- 2.Studienabschnitt (4 Semester, 47 SWS für Allgemeine Mathematik und 49 SWS für Numerische Mathematik

- 3.Studienabschnitt (4 Semester, 36 SWS für Allgemeine Mathematik und 34 SWS für Numerische Mathematik

Für Spezialisierungen können im 3. Studienabschnitt 26 SWS (Allgemeine Mathematik) bzw. 18 SWS (Numerische Mathematik) gewählt werden. Zusätzlich können beide Zweige 12 SWS für freie Wahlfächer wählen. Zum Abschluss muss eine Diplomarbeit innerhalb von 6 Monaten geschrieben werden.

Für das Lehramtsstudium ist eine Kombination von 2 Fächern obligatorisch. Für das Fach Mathematik sind 100 SWS in einer Regelstudienzeit von 9 Semestern vorgesehen. In diesen 100 SWS sind 68 SWS für Mathematik, 7 SWS für allgemeine Pädagogik, 15 SWS für Pädagogik im Mathematikunterricht enthalten. 10 SWS können als freie Wahlfächer gewählt werden. Von den 68 SWS für Mathematik können 8 SWS frei gewählt werden. Das Studium gliedert sich in 2 Abschnitte:

- 1. Studienabschnitt umfasst 5 Semester

- 2. Studienabschnitt umfasst 4 Semester

Der überwiegende Teil der Pädagogik findet im 2. Studienabschnitt mit 13 SWS statt. Zum Abschluss muss eine Diplomarbeit geschrieben werden, in einem der beiden Studienfächer nach Wahl.

\section{Prüfungsverfahren}

Aus finanziellen Gründen werden nur die 2 Einführungskurse „Lineare Algebra“ und „Analysis“ von Tutorials begleitet. Übungen werden obligatorisch angeboten.

Das Lehramtsstudium kann nach Meinung des Instituts nur schwer in der vorgesehenen Zeit von 9 Semestern absolviert werden, da der Student noch ein zweites Fach neben Mathematik belegen muss. 
Für jedes Unterrichtsfach muss eine Einzelprüfung abgelegt werden. Diese Einzelprüfungen können auf Wunsch der/des Studierenden auch als Fachprüfungen oder Gesamtprüfungen erfolgen. Ausgenommen von Fach- oder Gesamtprüfungen sind Lehrveranstaltungen mit immanentem Prüfungscharakter und Lehrveranstaltungen, die im Studienplan als Aufnahmevoraussetzungen anderer Lehrveranstaltungen genannt sind. Bereits abgelegte Prüfungen sind anzurechnen. Die Studentin/der Student hat eine Diplomarbeit aus einem der beiden gewählten Unterrichtsfächer zu verfassen. Auch wenn das 2. Fach an einer anderen Fakultät oder Universität gewählt wurde, ist insgesamt nur eine Diplomarbeit zu verfassen.

Im ersten Studienabschnitt sind 23 Prüfungen abzulegen, 7 davon betreffen Lehrveranstaltungen mit immanentem Prüfungscharakter. Im zweiten Prüfungsabschnitt werden 27 Prüfungen verlangt, 9 davon für Lehrveranstaltungen mit immanentem Prüfungscharakter.

Beide Studienzweige des Diplomstudienganges können in der vorgesehenen Zeit von 10 bzw. 4 Semestern absolviert werden. Für jedes Wahlpflichtfach und jedes Freie Wahlfach müssen Prüfungen abgelegt werden. Diese können mündlich oder schriftlich erfolgen.

Übungsklassen, Seminare und ähnliche Klassen haben immanenten Prüfungscharakter. Im ersten Studienabschnitt werden 9 Prüfungen verlangt, 5 davon betreffen Lehrveranstaltungen mit immanentem Prüfungscharakter. Im zweiten Studienabschnitt werden 17 und 8 Prüfungen für den Zweig Allgemeine Mathematik und 18 und 8 für den Zweig Numerische Mathematik verlangt und im dritten Studienabschnitt 11 und 4 für Allgemeine Mathematik, bzw. 10 und 2 für Numerische Mathematik. Das Schlussexamen findet vor einem Komitee statt und betrifft 2 Bereiche der Mathematik.

Beim Doktoratsstudium variiert die Anzahl der Prüfungen (abhängig von der Wahl des Studenten) im allgemeinen werden 5 verlangt. Das Schlussexamen findet vor einem Komitee statt und betrifft die Verteidigung der Doktorarbeit, ein Hauptthema und ein Thema aus dem Unterrichtsstoff des vom Studenten gewählten Wahlfaches.

\section{Studienerfolg}

Die Zahl der abgelegten Vordiplomprüfungen war im Zeitraum 2001-2003 bei 131 im Lehramtsstudiengang, 17 im Diplomstudiengang.

Die Zahl der abgelegten Diplomprüfungen im gleichen Zeitraum: 86 im Lehramtsstudiengang, 6 im Diplomstudiengang und 7 im Doktoratsstudium. 
Das Durchschnittsalter der AbsolventInnen lag im Lehramtsstudiengang zwischen 28 Jahren (in 2001) und 26 Jahren (in 2003). Im Diplomstudiengang zwischen 33 (2001) und 26 Jahren (2003) und im Doktoratsstudium zwischen 30,9 (2001) und 29,4 (2003) Jahren.

Die durchschnittliche Studiendauer betrug im

- Lehramtsstudiengang zwischen 13,4 Semester (2001) und 12,4 Semester(2003)

- Diplomstudiengang zwischen 19,2 Semester (2001) und 10,4 Semester (2003)

- Doktoratsstudium zwischen 5,8 Semester (2001) und 6,0 Semester (2003).

\section{Qualitätssicherung}

Auf Universitätsniveau existiert eine studentische Evaluierungsgruppe für Kurse. Die Studienkommission überprüft die Lehrleistungen und ist Forum für Diskussionen über Verbesserung der Lehre.

Ein Defizit für die Studienzeitverlängerung im Lehramtsstudium sieht das Institut darin, dass die AbsolventInnen zusätzlich ein 2. Studienfach neben Mathematik studieren müssen. Die Studienpläne dieser Studienfächer sind nicht gut koordiniert. Ein weiterer Grund für die Studienzeitverzögerung liegt in der Anzahl der Prüfungswiederholungen bei mündlichen Prüfungen. Im allgemeinen können Prüfungen 3 mal wiederholt werden. Die Situation wird dadurch verschlechtert, dass von einigen Mitarbeitern negative Prüfungsresultate der mündlichen Prüfungen nicht notiert werden.

\section{Gesamteinschätzung des Institutes}

Die Stärken und Schwächen der Ausbildung werden vom Institut wie folgt beurteilt:

\section{Stärken}

- starke Forschungsorientierung

Schwächen

- Begrenzung der Semesterwochenstunden für das derzeitige Diplomstudium

- Verzögerungen verursacht durch fehlende Regelungen, die den Studenten klare Anweisungen geben, in welchem Zeitraum Prüfungen abzulegen sind 
Alle Kurse des Studienplanes werden regelmäßig angeboten. Im neuen Bakkalaureat-MasterStudium würde sich die Fakultät mehr projektorientierte Seminare wünschen. Die Einführung eines Studentenberaters könnte hilfreich sein.

Das Institut hat schon damit begonnen, das Diplomstudium in ein Bakkalaureat-Master-

Studium umzuwandeln. Bezüglich der Neuorientierung des Lehramtsstudienganges haben die Diskussionen angefangen. 


\subsection{Universität Innsbruck}

\section{Naturwissenschaftliche Fakultät}

\section{Teil A: Forschung, Wissenschaftlicher Nachwuchs}

\section{Institut für Mathematik}

Das Institut verfügt über 23 Arbeitsräume (15 für Institutsmitglieder, 4 für Projektmitarbeiter und Dissertanten, 1 Sekretariat, 2 Seminarräume, 1 Prüfungszimmer), 1 Computerlabor (15 Arbeitsplätze); 1 Workstationraum (3 Arbeitsplätze); 1 Archivraum (kl. Handbibliothek), 1 Lagerraum. An Geräten stehen zur Verfügung: 43 Computerarbeitsplätze, 4 Notebooks, 2 Beamer, 4 Messgeräte für farbmetrische Untersuchungen.

Das Institut verfügt über folgende Finanzmittel:

\begin{tabular}{|l|r|r|r|}
\hline in $€$ & 2001 & 2002 & 2003 \\
\hline laufende Ausgaben & $\mathbf{1 7 . 0 0 0}$ & $\mathbf{1 6 . 6 0 0}$ & $\mathbf{1 2 . 5 0 0}$ \\
\hline Investitionen (Bundesmittel) & 47.000 & 18.000 & 22.000 \\
Investitionen (Drittmittel) & 1.400 & 4.800 & 3.900 \\
& & & \\
\hline Einnahmen Bundesmittel & $\mathbf{6 3 . 0 0 0}$ & $\mathbf{3 4 . 0 0 0}$ & $\mathbf{3 4 . 0 0 0}$ \\
\hline Drittmittel über die Univ. & 5.000 & 5.000 & 1.700 \\
Drittmittel FWF & 10.500 & 34.800 & 49.500 \\
\hline
\end{tabular}

Das Institut beurteilt seine Ausstattung als gut. Es gibt 17 Stellen (3 Professoren, 8 Dozenten, 5 wiss. Mitarbeiter, 1 Sekretariat), die aus Haushaltsstellen finanziert sind und 1 Stelle über Drittmittel.

Eine Professorenstelle ist seit März 2003 vakant. Die Berufungskommission für eine Professur für Stochastik hat im Dezember einen Ausschreibungstext verfasst, wurde aber dann (wie alle anderen Nachbesetzungen von Professorenstellen an der Universität Innsbruck) auf Grund der beginnenden Umstrukturierung nach UG 2002 und der schwierigen finanziellen Lage der Universität ,eingefroren“.

Die mathematische Bücher- und Zeitschriftenbibliothek ist in die naturwissenschaftliche Fachbibliothek integriert und sei sehr gut ausgestattet. Die Wartung der PCs und die Versorgung mit Software erfolgt problemlos über den ZID (Zentraler Informatikdienst der Universität Innsbruck). 


\section{Publikationen}

Das Institut hat im Zeitraum 2001-2003 28 Publikationen für Zeitschriften und Bücher erstellt und 37 Vorträge gehalten.

\section{Wissenschaftlicher Nachwuchs}

In den letzten 5 Jahren wurden 3 Promotionen abgelegt. Die Doktoranden waren durchschnittlich 30 Jahre alt. Habilitationen gab es in diesem Zeitraum keine.

\section{Kooperation in der Forschung}

Das Institut arbeitet in folgenden Kooperationsbeziehungen:

Interdisziplinäre Zusammenarbeit mit den Instituten für Meteorologie, für Gerichtliche Medizin und für Informatik; Acciones Integradas mit Universitäten Madrid, Valladolid und Sevilla, Zusammenarbeit mit MathematikerInnen an den Universitäten Valladolid, Sevilla, Madrid und Linz, Kooperation mit Licht-planungsfirmen

\section{Anerkennung der Leistungen des Instituts}

Die Mitglieder des Instituts waren in verschiedener Funktion als Gutachter und Herausgeber von Zeitschriften oder als Mitglieder von Programm-Komitees im In- und Ausland tätig.

Das Institut für Mathematik ist für die Studienrichtungen Technische Mathematik und Lehramtsstudium Mathematik verantwortlich. Studieninformation (siehe Broschüre „Mathematikstudium an der Universität Innsbruck“), Planung des Lehrangebots, Arbeit in den Studienkommissionen usw. erfordern nach Aussage des Instituts einen erheblichen Arbeitsaufwand. Darüber hinaus hat das Institut intensiv am Aufbau des Instituts für Informatik und der Studienrichtung Informatik mitgearbeitet.

\section{Institut für Technische Mathematik, Geometrie und Bauinformatik}

Für das Institut für Technische Mathematik, Geometrie und Bauinformatik ist bemerkenswert, dass es keine eigene Studienrichtung betreut. Wichtig ist die Schwerpunktbildung in Angewandter Mathematik mit intensiven Kooperationen im Ingenieurbereich.

\section{Raum-, Sach-, Personalausstattung}

Das Institut verfügt über Büro- und Technikräume auf einer Gesamtnutzfläche von $690,3 \mathrm{~m}^{2}$. An Geräteausstattung ist vorhanden: 1 Server, pro Mitarbeiter 1 PC. Es verfügt über folgende 
Finanzmittel:

\begin{tabular}{|l|r|r|r|}
\hline in $€$ & 2001 & 2002 & 2003 \\
\hline laufende Ausgaben & $22.949,00$ & $17.385,00$ & $14.654,00$ \\
\hline Investitionen (Bundesmittel) & $73.207,00$ & $21.879,00$ & $3.852,00$ \\
\hline Einnahmen Bundesmittel & $98.049,00$ & $38.670,00$ & $16.444,00$ \\
\hline Drittmittel über die Universität & 201,00 & 24,00 & 72,00 \\
Drittmittel FWF & $42.000,00$ & $51.000,00$ & $49.000,00$ \\
Sonstige Drittmittel & $1.500,00$ & $4.800,00$ & $20.700,00$ \\
\hline
\end{tabular}

Das Institut verfügt über 16 Stellen (4 Professoren, 4 Dozenten, 7 wiss. Personal, 3 nichtwiss. Personal), die aus Haushaltsstellen finanziert werden und 1 Stelle über Drittmittel.

1 Professorenstelle (Kat. I) ist unbesetzt; 1 Professorenstelle (Kat. II), gegen Einzug einer freiwerdenden Assistenten- oder Dozentenstelle besetzbar. Beide Stellen werden derzeit seitens des Rektorates aus budgetären Gründen nicht besetzt.

Die Universität Innsbruck besitzt eine sehr gut ausgebaute mathematische Zeitschriftenbibliothek. Die Fachbibliothek am Institut für Technische Mathematik, Geometrie und Bauinformatik ist ebenfalls ausgezeichnet bestückt und bisher finanziell gut ausgestattet gewesen.

\section{Lehrexporte und-importe}

Neben der Hauptaufgabe in der Ingenieurausbildung wirken Mitarbeiter des Instituts für Technische Mathematik, Geometrie und Bauinformatik in der Lehre im Fach Mathematik (Diplom, Lehramt und Doktorat) der Universität Innsbruck mit. Die anteilige Aufteilung ist etwa 75\% für Ingenieurausbildung, 25\% für Mathematikausbildung.

\section{Publikationen}

Das Institut hat im Zeitraum 2001-2003 51 Veröffentlichungen in referierten Zeitschriften publiziert, 60 Besprechungen von Büchern und Artikel verfasst und 107 Vorträge, davon 53 auf Einladung, gehalten.

\section{Wissenschaftlicher Nachwuchs}

In den letzten 3 Jahren wurden am Institut 6 Promotionen abgelegt (2 von Externen). Es wurden 3 Diplomarbeiten im Bauingenieurwesen betreut (weitere 4 Diplomarbeiten aus Mathematik wurden beim Fachbereich gezählt). Habilitationen gab es in diesem Zeitraum keine. 
Es werden Spezialvorlesungen für Diplomanden und Dissertanten aus dem Bereich der Technischen Wissenschaften angeboten und Diplomarbeiten und Dissertationen aus diesem Bereich betreut.

\section{Personalfluktuation}

Ab 2005 wird eine gravierende Umstrukturierung umgesetzt, bei der 4 Mitarbeiter des Institutes für Technische Mathematik, Geometrie und Bauinformatik an die neue gegründete Fakultät für Mathematik-Informatik-Physik umgesetzt werden.

\section{Kooperationen in der Forschung}

Das Institut unterhält zahlreiche Kooperationsbeziehungen zu Universitäten im In- und Ausland (z.B. Università di Torino, University of California) und Instituten (LARM: Laboratorio di Robotica e Meccatronica, Cassiono)

Anerkennung der Leistungen des Instituts

Die Mitglieder des Instituts waren in verschiedener Funktion als Gutachter und Herausgeber von Zeitschriften im In- und Ausland tätig. Zwei wichtige internationale Preise gingen an Wissenschaftler aus dem Institut.

\section{Arbeitsgruppe Infmath Imaging, Institut für Informatik}

Raum- ,Sach-, Personalausstattung

Die Arbeitsgruppe verfügt über 4 Räume auf einer Nutzfläche von $108 \mathrm{~m}^{2}$. Es gibt 15 PCs, 6 Notebooks, 1 Cluster mit 18 CPUs, 3 File-Server, 6 Server, 7 HP-Laserjet-Drucker, 1 Xerox Farbdrucker, 1 Scanner.

Die Arbeitsgruppe verfügt über folgende Finanzmittel:

\begin{tabular}{|l|r|r|r|}
\hline in $€$ & 2001 & 2002 & 2003 \\
\hline laufende Ausgaben & 105.637 & 105.386 & 61.625 \\
\hline Drittmittel FWF & 145.346 & 145.346 & 200.149 \\
\hline
\end{tabular}

Die Arbeitsgruppe hat 6,5 Stellen(1 Professur, 3,5 wiss. Personal, 2 nichtwiss. Personal), die über Haushaltsmittel finanziert werden und 4,5 Stellen aus Dritt- und Sondermitteln $(0,5)$. 


\section{Publikationen}

Von Mitgliedern der Arbeitsgruppe wurden insgesamt 28 Veröffentlichungen im Zeitraum von 3 Jahren publiziert in Zeitschriften und Proceedingsbänden. Es wurden 41 Vorträge gehalten, 22 davon auf Einladung.

\section{Wissenschaftliches Personal}

In den letzten 5 Jahren wurde 1 Promotion abgelegt. Habilitationen gab es keine.

Doktoranden werden mit 4 - 6 Wochenstunden für Mathematik für Informatiker in die Lehre einbezogen. 2-3 Doktoranden mit 4 Wochenstunden im Bakkalaureatseminar.

\section{Kooperation in der Forschung}

Die Arbeitsgruppe unterhält Kooperationsbeziehungen zu GE Medical Systems, Kretztechnik AG, Kompetenzzentrum IMCC, Linz

Anerkennung der Leistungen der Arbeitsgruppe

Einige Mitglieder der Arbeitsgruppe waren Gutachter für verschiedene Zeitschriften. 


\section{Teil B: Lehre und Studium am Fachbereich Mathematik der Universität Inns- bruck}

Ausgangslage

Der Fachbereich Mathematik bietet ein Diplomstudium Technische Mathematik (mit 10 Semestern) und ein Lehramtsstudium Mathematik (mit 9 Semestern für zwei Unterrichtsfächer sowie allgemeinpädagogische und schulpraktische Ausbildung) an. Daran anschließend ein Doktoratsstudium mit (4 Semestern); des weiteren alle Lehrveranstaltungen aus Mathematik (einschließlich Geometrie) in den Studienrichtungen Informatik, Bauingenieurwesen, Physik, Meteorologie und Geophysik, Architektur, Chemie, Erdwissenschaften.

\section{Allgemeine Ziele}

Das Diplomstudium Technische Mathematik bereitet auf eine Tätigkeit als Mathematikerin/Mathematiker in Industrie, Wirtschaft und Forschung vor. Am Ende des Studiums sollen die Studierenden in der Lage sein, komplexe Problemstellungen zu analysieren, mathematisch zu modellieren und Verfahren zu ihrer Lösung zu entwickeln.

Dazu werden im Studium das kreative und folgerichtige Denken geschult und die grundlegenden Teilgebiete und wichtige Algorithmen der Mathematik erlernt. Außerdem ist die Fähigkeit zu erwerben, sich weiteres Wissen aus der Fachliteratur anzueignen und mit Experten anderer Fachgebiete zusammenzuarbeiten.

Die gebundenen Wahlfächer ermöglichen den Studierenden, Schwerpunkte in einigen Teilgebieten der Pflichtfächer zu setzen sowie Anwendungsgebiete der Mathematik kennen zu lernen. Aus diesen Gebieten werden solche Lehrveranstaltungen empfohlen, die mathematische Probleme motivieren und die Kommunikation mit Anwendern erleichtern.

Für das Diplomstudium Technische Mathematik ist die inhaltliche Schwerpunktbildung für die Studierenden individuell durch entsprechende Wahl des Wahlfaches (im Ausmaß von 28 Semesterstunden) im zweiten Studienabschnitt möglich. Die Wahlfächer sind „Algebra und Diskrete Mathematik“, „Analysis und Stochastik“, „Bauingenieurwesen“, „Biologie und Medizin“, „Chemie“, „Informatik“, „Physik“ und „Wirtschaftswissenschaften und Statistik“. Zusätzlich können freie Wahlfächer im Ausmaß von 20 Semesterstunden beliebig gewählt werden.

Ziel des Lehramtsstudiums ist die wissenschaftliche Berufsvorbildung für das Lehramt an Höheren Schulen in fachlicher, fachdidaktischer, pädagogischer und schulpraktischer Hin- 
sicht. Durch eine solide wissenschaftliche Grundausbildung, orientiert am Wissensstand der beteiligten Disziplinen und an der Lehrplanentwicklung an Österreichs Schulen, sollen die Absolventinnen und Absolventen in die Lage versetzt werden, sich in ihren Berufsfeldern flexibel und kompetent zu bewähren. Im universitären Lehramtsstudium sollen dabei die Eigeninitiative und Selbstorganisation, ein kritisches Bewusstsein, Kooperation und Teamfähigkeit sowie Leistungsbereitschaft besonders gefördert werden.

\section{Praxisbezug}

Im Diplomstudium Technische Mathematik gab es alle vier Semester eine Lehrveranstaltung von Praktikern, im Lehramtsstudium jedes Semester eine zweistündige Vorlesung über Methoden des Mathematikunterrichts durch erfahrene LehrerInnen an Höheren Schulen.

Der überwiegende Teil der Lehrenden im Fachbereich hält die Aufteilung des Diplomstudiums Technische Mathematik in ein Bakkalaureat- und Magisterstudium nicht für sinnvoll. Trotzdem wird, den Vorgaben von UG 2002 und Universitätsleitung folgend, diese Aufteilung in den nächsten zwei Jahren durchgeführt.

\section{Personelle Ressourcen}

Die Lehrkapazität des festen Lehrkörpers (136 SWS (Semesterwochenstunden), 70 SWS wiss. Mitarbeiter, ca. 25 SWS externe Lehrbeauftragte) umfasst ca. 230 SWS.

Das tatsächliche Lehrangebot lag durchschnittlich pro Semester für:

Technische Mathematik: 45 SWS im ersten und 30 SWS im zweiten Studienabschnitt.

Lehramtsstudium Mathematik: 15 SWS im zweiten Studienabschnitt (die übrigen Lehrveranstaltungen finden gemeinsam mit dem Diplomstudium Technische Mathematik statt).

Es entfallen auf die Zahl der externen Lehrbeauftragten nach Lehrgebieten:

- 6 SWS für Didaktik der Mathematik

- 2 SWS pro Studienjahr für Datennetze und Betriebssysteme

- 2 SWS pro Studienjahr für Mathematik in der Industrie

Alle übrigen Lehrbeauftragten wirken im Lehrbetrieb für die Studienrichtungen Informatik und Bauingenieurwesen mit.

Der Fachbereich bietet Service-Lehrveranstaltungen für folgende Studienrichtungen an:

- Studienrichtung Informatik (115 SWS)

- Studienrichtungen Bauingenieurwesen und Architektur (115 SWS)

- Studienrichtung Physik (21 SWS) 
- Studienrichtung Meteorologie und Geophysik (22 SWS)

- Studienrichtung Chemie (4 SWS)

- Studienrichtung Erdwissenschaften (3 SWS)

Lehrangebote anderer Studienrichtungen wurden von einigen Studierenden der Technischen Mathematik im Rahmen der Wahlfächer in Anspruch genommen.

\section{Sachausstattung}

Die räumliche, sächliche und finanzielle Ausstattung wird als gut bezeichnet. Es gibt 3 Arbeitsplätze mit Workstations für DiplomandInnen und DissertantInnen. 15 Arbeitsplätze mit PCs im EDV-Labor des Instituts für Mathematik, die EDV-Labors des Instituts für Informatik, der Baufakultät und des ZID (Rechenzentrum der Universität) können mitbenutzt werden. Studienanfänger $(\mathrm{m} / \mathrm{w})$

\begin{tabular}{|c|c|c|c|c|c|}
\hline $\begin{array}{l}\text { Studiengang/ } \\
\text { angestrebter Abschluss }\end{array}$ & 1999 & 2000 & 2001 & 2002 & 2003 \\
\hline \multicolumn{6}{|l|}{ Diplomstudium Mathematik } \\
\hline Erstes Fachsemester & $25 / 34$ & $3 / 10$ & k.A. & k.A. & k.A. \\
\hline davon Erstzugelassene & $14 / 25$ & $0 / 3$ & k.A. & k.A. & k.A. \\
\hline \multicolumn{6}{|l|}{ Diplomstudium Technische Mathematik } \\
\hline Erstes Fachsemester & k.A. & $19 / 47$ & $21 / 41$ & $29 / 84$ & $22 / 48$ \\
\hline davon Erstzugelassene & k.A. & $12 / 29$ & $15 / 24$ & $10 / 31$ & $20 / 34$ \\
\hline \multicolumn{6}{|l|}{ Lehramtsstudium Mathematik } \\
\hline davon Erstzugelassene & $20 / 25$ & $16 / 22$ & $14 / 14$ & $10 / 10$ & $22 / 23$ \\
\hline & $11 / 16$ & $13 / 16$ & $8 / 4$ & $8 / 6$ & $18 / 16$ \\
\hline
\end{tabular}

Studienanfänger in anderen Studienrichtungen, die Lehrleistungen des Fachbereichs in Anspruch nehmen, durchschnittlich pro Jahr:

- Studienrichtung Informatik: ca. 150

- Studienrichtung Bauingenieurwesen: ca. 80

- Studienrichtung Architektur: ca. 200 
- Studienrichtung Physik: ca. 60

- Studienrichtung Meteorologie und Geophysik: ca. 20

- Studienrichtung Chemie: ca. 40

- Studienrichtung Erdwissenschaften: ca. 20.

\section{Studien- und Prüfungsordnung}

Eine verpflichtende Abfolge der Lehrveranstaltungen gibt es nicht, sehr wohl aber einen „empfohlenen Studiengang“, der eine sinnvolle Reihenfolge vorgibt.

Alle Vorlesungen werden durch verpflichtend vorgeschriebene Proseminare (in mehreren Gruppen mit maximal 20 Studierenden) begleitet (siehe Studienplan). Zu den Vorlesungen im ersten Studienjahr gibt es Tutorien (je zwei Semesterstunden).

Es entfallen auf den Diplomstudium Technische Mathematik:

Prüfungsfächer im ersten Studienabschnitt („Grundstudium“, 4 Semester): 68 Semesterstunden Pflichtlehrveranstaltungen, keine Wahlfächer.

Prüfungsfächer im zweiten Studienabschnitt („Hauptstudium“, 6 Semester): 45 Semesterstunden Pflichtlehrveranstaltungen, 28 Semesterstunden ,gebundene Wahlfächer“, 20 Semesterstunden „freie Wahlfächer“ (keinem Studienabschnitt zugeordnet). Kernfächer sind die Pflichtfächer im 2. Studienabschnitt: Algebra und Geometrie, Analysis, Numerische Mathematik, Wahrscheinlichkeitstheorie und Mathematische Statistik.

Auf das Lehramtsstudium Mathematik:

Prüfungsfächer im ersten Studienabschnitt („Grundstudium“, 4 Semester): 32 Semesterstunden Pflichtlehrveranstaltungen, keine Wahlfächer.

Prüfungsfächer im zweiten Studienabschnitt („Hauptstudium“, 5 Semester): 38 Semesterstunden Pflichtlehrveranstaltungen, 3 Semesterstunden ,gebundene Wahlfächer“, 9 Semesterstunden „freie Wahlfächer“ (keinem Studienabschnitt zugeordnet). Kernfächer sind die Pflichtfächer im 2. Studienabschnitt: Algebra und Geometrie, Analysis und Stochastik, Fachdidaktik, Horizonte der Mathematik.

Die Einführungsvorlesungen wurden während der letzten Jahre von Professoren oder Dozenten gehalten, die Verteilung der Lehrleistungen nach dienstrechtlichen Kategorien ist von Jahr zu Jahr verschieden.

Es werden alle in der Studien- und Prüfungsordnung vorgesehene Lehrgebiete regelmäßig angeboten. Die meisten Pflichtlehrveranstaltungen des zweiten Studienabschnittes werden nur 
im Zweijahresrhythmus angeboten, die Studierenden werden aber rechtzeitig darauf aufmerksam gemacht.

Der Fachbereich beteiligt sich am Sokrates-Programm der EU mit 9 europäischen Universitäten, ca. 4 Studierende pro Jahr nehmen daran teil.

\section{Prüfungsverfahren}

Der Großteil der Prüfungen wird in Form von Lehrveranstaltungsprüfungen vor Einzelprüfern abgelegt. Wiederholungen bei kommissionellen Prüfungen sind selten. Die Termine für den Großteil der Prüfungen werden kurzfristig persönlich mit dem Prüfer vereinbart. Die Mindestzahl der Prüfungstermine und die Zusammenstellung der Prüfungskommissionen sind gesetzlich geregelt (UG 2002, früher UniStG 1993):

Prüfungstermine sind für den Anfang, für die Mitte und für das Ende jeden Semesters anzusetzen.

Es gibt drei Prüfungswiederholungen. Darüber hinaus ist keine weitere Wiederholung zulässig. Kommissionelle Gesamtprüfungen müssen zur Gänze wiederholt werden, wenn ein Fach negativ beurteilt wurde.

\section{Leistungsanforderungen}

Für beide Studiengänge sind über alle vorgeschriebenen Lehrveranstaltungen Prüfungen abzulegen. Das Studium wird durch eine Diplomarbeit und eine kommissionelle Diplomprüfung abgeschlossen. Im Lehramtsstudium ist zusätzlich am Ende des ersten Studienabschnitts eine kommissionelle Prüfung abzulegen.

Der Anteil der Teilzeitstudierenden wird grob auf 20\% geschätzt. Genaue Zahlen dazu sind nicht bekannt. Nach UG 2002 umfasst das Arbeitsjahr eines Vollzeitstudierenden 1500 volle Arbeitsstunden. Wer für das Studium weniger Zeit aufwenden kann, muss mit entsprechend längerer Studiendauer rechnen. 


\section{Studienerfolg}

Zahl der abgelegten Zwischenprüfungen/Vordiplome der letzten 5 Prüfungsjahre

\begin{tabular}{|l|r|r|r|r|r|}
\hline Studiengang & $1999 / 00$ & $2000 / 01$ & $2001 / 02$ & $2002 / 03$ & $2003 / 04$ \\
\hline Mathematik Diplom & 615 & 983 & 788 & 743 & 1011 \\
\hline Mathematik LA & 609 & 593 & 511 & 423 & 953 \\
\hline Bauingenieur Diplom & 667 & 870 & 878 & 1020 & 1185 \\
\hline Architektur Diplom & 590 & 792 & 937 & 1161 & 1248 \\
\hline Physik & 129 & 170 & 138 & 174 & 92 \\
\hline Physik LA & 28 & 13 & 4 & 5 & 3 \\
\hline Informatik & 0 & 0 & 936 & 1038 & 1316 \\
\hline Meteorologie und Geophysik & 103 & 90 & 49 & 92 & 93 \\
\hline Chemie (Diplom und LA) & 78 & 79 & 7 & 77 & 59 \\
\hline Erdwissenschaften(Geogr + LA) & 21 & 14 & 2 & 38 & 32 \\
\hline Doktorat Techn. Wissenschaften & 0 & 1 & 0 & 13 & 11 \\
\hline Doktorat Naturwissenschaften & 3 & 5 & 2 & 13 & 6 \\
\hline Sonstige & 5 & 20 & 14 & 18 & 101 \\
\hline
\end{tabular}

Zahl der AbsolventInnen der letzten 5 Prüfungsjahre

\begin{tabular}{|l|r|r|r|r|r|}
\hline Studiengang & $1998 / 99$ & $1999 / 00$ & $2000 / 01$ & $2001 / 02$ & $2002 / 03$ \\
\hline $\begin{array}{l}\text { Diplomstudium Mathematik } \\
\text { Diplomstudium Technische Mathematik }\end{array}$ & k.A. & k.A. & k.A. & k.A. & 2 \\
Lehramtsstudium Mathematik & $15(9)$ & $18(4)$ & $15(8)$ & $20(8)$ & $14(8)$ \\
(in Klammer: Mathematik als Hauptfach) & & & 11 & & \\
Doktoratsstudium & & 2 & 2 & 3 & 4 \\
\hline
\end{tabular}

\section{Qualitätssicherung}

Die Qualität der Lehre wird durch das Vizerektorat für Evaluation (bzw. jetzt für Lehre und Studierende) in Form von in der Lehrveranstaltung auszufüllenden Fragebögen regelmäßig 
durchgeführt. Die Ergebnisse werden - sofern die Veröffentlichung nicht durch den Lehrveranstaltungsleiter untersagt wird - im elektronischen Lehrzielkatalog publiziert.

Über die Verbesserung von Ausbildung und Lehrleistungen wird in Beratungen in der Studienkommission und in den Institutsversammlungen regelmäßig diskutiert.

Durch Tutorien am Studienbeginn und Probeklausuren nach einem Studienmonat wird den allgemeinen Defiziten der Studierenden entgegengetreten. Für Studierende des Bauingenieurwesens werden Vor- und Aufbaukurse in Darstellender Geometrie, CAD und Mathematik angeboten. Die Rahmenbedingungen für das Studium sind derzeit gut (überschaubarer Studienbetrieb, regelmäßiges Lehrangebot, keine wesentlichen Wartezeiten bei Prüfungen, ausreichendes Angebot an Betreuern von Diplomarbeiten, ...).

Auf Langzeitstudierende und Prüfungsrücktritte wird oft durch persönliche Gespräche und Beratung durch Lehrende reagiert. Die Anzahl von Studienabbrechern ist im ersten Studienjahr sehr hoch (ca. 50\%), später sehr gering. Der häufigste Grund für einen Studienabbruch liegt darin, dass viele Studierende ihr Interesse an der Mathematik zu hoch eingeschätzt haben. Es wird versucht, durch gezielte Information an MaturantInnen (am Tag der offenen Tür, bei der Berufsinformationsmesse, durch Versenden der Broschüre „Mathematikstudium an der Universität Innsbruck“ und durch das Angebot von Einzelgesprächen mit Institutsmitgliedern) die Chancen auf die richtige Studienwahl zu verbessern.

\section{Gesamteinschätzung des Fachbereichs}

Durch die Neuorganisation der Universität sind einschneidende organisatorische Veränderungen im Gang gekommen: Seit Oktober 2004 gehört das Institut für Mathematik (der naturwiss. Fakultät) der Fakultät für Mathematik, Informatik und Physik an. Ein Teil des Personals des Instituts für Technische Mathematik (der Baufakultät) wurde an das Institut für Mathematik verlegt. Es gibt keine Studienkommission mehr. Das Studium der Technischen Mathematik soll zweigliedrig (Bakkalaureat- und Magisterstudium) organisiert werden. 


\subsection{Universität Linz}

\section{Teil A: Forschung, Wissenschaftlicher Nachwuchs}

\section{Institut für Algebra}

\section{Raum-, Sach-, Personalausstattung}

Die räumliche Ausstattung ist knapp bemessen. Für 8 Personen (eine Person mit 1/2 Beschäftigungsverhältnis) stehen 4 Räume zur Verfügung. Eigene Räume für Gastprofessoren/Gastforscher gibt es nicht. Die infrastrukturelle Ausstattung ist gut. Durch den Linux PCCluster mit gemeinsamem Server haben alle MitarbeiterInnen Zugang zu den wichtigsten Dokumenten und Programmen.

Das Institut verfügt über folgende Finanzmittel:

\begin{tabular}{|l|r|r|r|}
\hline \multicolumn{1}{|c|}{$€$} & 2001 & 2002 & 2003 \\
\hline Laufende Ausgaben (o.Dot) & 6.740 & 6.110 & 7.834 \\
\hline Investitionen & & & \\
Bundesmittel (ao.Dot) & 2.180 & 6.804 & 4.852 \\
Einnahmen Bundesmittel & 7.892 & 12.274 & 12.612 \\
Drittmittel FWF & & 70.876 & 135.200 \\
\hline
\end{tabular}

In den letzten 3 Jahren ist ein Forschungsprojekt über FWF für die Dauer von 36 Monaten mit einem Betrag von $€ 241.259,90$ finanziert worden.

Die personelle Ausstattung wird vom Institut als sehr knapp angesehen. Das Personaltableau weist folgende aus Haushaltsmitteln finanzierte Stellen aus: 1 Professor, 2 wissenschaftliches Personal, 4 Lehrbeauftragte; $1 / 2$ Stelle Sekretariat, 1/4 Stelle Technische Dienste. Zusätzlich aus Drittmitteln finanzierte Stellen: 3 wissenschaftliche Mitarbeiter auf Zeit.

Die Bibliothek ist die gemeinsame Bibliothek aller mathematischen Institute.

\section{Besondere Aktivitäten in der Lehre}

Doktoranden und Habilitanden werden bereits in die Lehre mit einbezogen. Eigenständige Publikationsleistungen werden bereits von Dissertanten erwartet. $75 \%$ des Personals in Forschungsprojekten sind Doktoranden. 


\section{Publikationen}

Im Zeitraum 2001-2003 hat das Institut 23 referierte Publikationen veröffentlicht, davon 10 in Zeitschriften, 3 in Tagungsbänden und 10 in Sammelwerken. Darüber hinaus gab es 2 Dissertationen und 5 Diplomarbeiten. Es wurden 72 wissenschaftliche Vorträge gehalten, davon 44 auf Einladung.

\section{Wissenschaftlicher Nachwuchs}

In den letzten 5 Jahren wurden 2 Promotionen (von Externen) abgelegt (in 2001). Die durchschnittliche Promotionsdauer betrug 3 Jahre, das Durchschnittsalter lag bei 27 Jahren. Im Zeitraum von 5 Jahren wurde 1 Habilitation abgelegt, die innerhalb von 6 Jahren geschrieben wurde, das Alter des Habilitanden war bei 33 Jahren.

\section{Personalfluktuation}

In den letzten 5 Jahren hat 1 Wissenschaftler das Institut aufgrund eines auswärtigen Rufes verlassen, dafür ist 1 Wissenschaftler neu berufen worden. Das Institut sieht die Schwierigkeiten in der Rekrutierung neuer Mitarbeiter darin, dass Angebote von Fachhochschulen verlockender sind.

\section{Kooperation in der Forschung}

Es bestehen zahlreiche Kooperationsbeziehungen zu Universitäten in China, Russland, USA, Polen, Deutschland, Australien wie z.B. Moscow State University: Partneruniversität, gemeinsames Buch; Ural State University (Ekatarinburg, Russland): Gemeinsame Bücher; Texas A\&M University (College Station, Texas): jahrzehntelange gemeinsame Forschung.

\section{Anerkennung der Leistungen des Instituts}

Die Mitglieder des Instituts waren in verschiedener Funktion als Gutachter im In- und Ausland tätig. Es wurde 1 Förderpreis für Arbeiten auf dem Gebiet der Algebra und 1 Ehrendoktorat der Ural State University, Ekatarinburg, Russland verliehen.

\section{Institut für Analysis}

Das Institut besteht aus den Abteilungen: Approximationstheorie und Dynamische Systeme, Finanzmathematik, Funktionsanalysis. 
Raum-, Sach-, Personalausstattung

Nach Ansicht des Institutes gibt es ein Defizite bei der Ausstattung mit Räumen. Es stehen 9 Räume (2 große und 7 kleine) für wissenschaftliche Mitarbeiter und 2 Sekretariatsräume zur Verfügung. Es gibt keine geeigneten Arbeitsplätze für Gäste.

Das Institut verfügt über folgende Finanzmittel:

\begin{tabular}{|ll|r|r|r|}
\hline$€$ & & 2001 & 2002 & 2003 \\
\hline Laufende Ausgaben & ca. & 13.000 & 13.000 & 13.217 \\
\hline Investitionen (Bundesmittel) & ca. & 4.000 & 3.500 & 2.800 \\
Investitionen (Drittmittel) & ca. & k.A. & 1.600 & 1.200 \\
\hline Einnahmen & & 0 & 0 & 54.000 \\
\multicolumn{1}{l|}{ Drittmittel über die Universität } & 140.000 & 140.000 & 210.000 \\
Drittmittel FWF & & & & \\
\hline
\end{tabular}

Anmerkung: Die finanzielle Zuteilung ist nicht mehr exakt rekonstruierbar, da bis 2001 bzw.

Ende 2003 noch andere Institute zum Institut für Analysis gehörten.

Die infrastrukturelle Ausstattung wird vom Institut als ausreichend angesehen. Das Institut verfügt über 17 PCs und 5 Laptops sowie Zugriff auf Rechnerkapazitäten anderer Institute.

Aus Haushaltsmitteln finanzierte Stellen gibt es insgesamt 11 (2 Professoren, 2 wiss. Mitarbeiter auf Zeit, 5 auf Dauer, 2 Sekretariate), aus Drittmitteln finanziert (incl. FWF) gibt es 6 Stellen auf Zeit. Die personelle Ausstattung wird vom Institut als sehr gut für die Abteilung Finanzmathematik angesehen, als eher schlecht für die Abteilung Approximationstheorie und Dynamische Systeme.

\section{Besondere Aktivitäten in der Lehre}

Es besteht eine enge Einbindung aller Mitarbeiter in größere Forschungsgruppen vor allem in das Johann-Radon-Institut und in die österreichischen Forschungsschwerpunkte und volle Einbeziehung der Doktoranden in die Lehre (Übungen für Doktoranden, Übungen und Vorlesungen für Habilitanden). Es wird darauf geachtet, dass vor der Habilitation ein Forschungsaufenthalt im Ausland ermöglicht wird. In zwei Fällen ist dieser Aufenthalt durch Schrödinger-Stipendien finanziert worden.

Es ist eine Spin-off-Firma gegründet worden: ArtIn Finance Vermögensverwaltung GmbH. 


\section{Publikationen}

Im Zeitraum 2001-2003 gab es 42 referierte Aufsätze, davon 38 in Zeitschriften und 4 in Tagungsbänden. Es wurde 37 Vorträge im internationalen Rahmen gehalten, davon 26 auf Einladung.

\section{Wissenschaftlicher Nachwuchs}

Die Zahl der Promotionen lag in den Jahren 1999 - 2003 bei 5, davon 3 von Stipendiaten. Die durchschnittliche Promotionsdauer betrug 2,5 Jahre. Das Durchschnittsalter der Doktoranden lag bei 27 Jahren. Es gab 3 Habilitationen im Zeitraum 1999-2003 mit einer durchschnittlichen Habilitationsdauer von 5 Jahren. Das Durchschnittsalter lag bei 32.

\section{Personalfluktuation}

In den letzten 5 Jahren haben 5 Wissenschaftler das Institut verlassen (davon 1 Ruf an eine andere Universität). Demgegenüber stehen 3 Neuzugänge (1 Professor, 2 Assistenten).

\section{Kooperation in der Forschung}

Das Institut hat mehrere Internationale Konferenzen und Workshop in Österreich ausgerichtet. Es unterhält internationale Kooperationsbeziehungen zu Universitäten in Frankreich, USA, Ungarn, Spanien, Belgien, Ukraine, Griechenland, Italien.

Einer der beiden Professoren (G. Larcher) ist Sprecher des FWF-Forschungsschwerpunktes „Number-Theoretic Algorithms and their Applications“.

\section{Anerkennung der Leistungen des Fachbereichs}

Die Mitglieder des Instituts waren in verschiedener Funktion als Gutachter im In- und Ausland tätig. Mehrere Institutsmitglieder sind mit Forschungspreisen ausgezeichnet worden z.B. Förderpreis der Österreichischen Mathematischen Gesellschaft, Rosa und Edmund HlawkaPreis der Österreichischen Akademie der Wissenschaften.

\section{Institut für Angewandte Geometrie}

\section{Raum-, Sach-, Personalausstattung}

Nach Meinung des Instituts sind die Kapazitätsgrenzen bei Raum- und Rechnerausstattungen erreicht. Das Institut verfügt über 3 Räume mit 4-5 Arbeitsplätzen, dazu ein im Rahmen des SFB angemieteter Raum mit 2-3 Arbeitsplätzen. Es sind 6 PCs (Linux) und 10 Laptops im Einsatz. Des weiteren steht ein 3D-Laserscanner (Minolta VIVID 300) zur Verfügung. Das Institut verfügt über folgende Finanzmittel: 


\begin{tabular}{|l|r|r|r|}
\hline$€$ & 2001 & 2002 & 2003 \\
\hline Laufende Ausgaben (Bundesmittel) & 4.000 & 4.100 & 5.300 \\
\hline Investitionen (Bundesmittel) & 15.000 & 14.000 & 0 \\
Investitionen (Drittmittel) & 16.000 & 20.000 & 16.000 \\
\hline Drittmittel über die Universität & 0 & 31.000 & 76.000 \\
davon Personalkosten & 0 & 26.000 & 52.000 \\
& & & \\
Drittmittel FWF & 56.000 & 76.000 & 76.000 \\
davon Personalkosten & 53.000 & 71.000 & 71.000 \\
\hline
\end{tabular}

Es gibt am Institut 3,5 Stellen (1 Professur (Kat.II), 2,5 wiss. Personal), die aus Haushaltsmitteln bezahlt werden und 4 Stellen (wiss. Personal) drittmittelfinanziert.

\section{Besondere Aktivitäten in der Lehre}

Die durch die Universität finanzierten Doktoranden und Post Docs werden in Übungen und Service-Lehrveranstaltungen eingesetzt.

\section{Publikationen}

Das Institut hat im Zeitraum 2001-2003 16 referierte Artikel verfasst, davon 10 in Sammelwerken. Es wurden 34 auf Foren internationale und nationale Vorträge gehalten, davon 17 auf Einladung.

\section{Wissenschaftlicher Nachwuchs}

Im Zeitraum 1999-2003 gab es 1 Promotion, deren Dauer 4 Jahre betrug. Habilitationen gab es im genannten Zeitraum keine.

\section{Personalfluktuation}

Im Zeitraum 2000 bis 2004 gab es 8 Neuzugänge, darunter 1 Professur.

\section{Kooperationen in der Forschung}

Der Institutsleiter ist Sprecher des geplanten FWF- Forschungsschwerpunkts „Industrial Geometry“ (Beginn: 04/2005). Das Institut unterhält Kooperationsbeziehungen zu Hochschulen in Frankreich, Hongkong und verschiedenen Universitäten Österreichs sowie zu außeruniversitären Forschungseinrichtungen und Industrieunternehmen in Norwegen, Frankreich und Deutschland. Das Institut hat 2003 einen internationalen Workshop in Österreich ausgerichtet. Bei Diplomarbeiten und in der Forschung arbeitet es eng mit Industriepartnern zusammen. 
Anerkennung der Leistungen des Fachbereichs

Der Institutsleiter ist Referee für zahlreiche Zeitschriften und Mitveranstalter von Konferenzen, darunter Associate Editor von Computer Aided Geometrie Design, Mitglied des Editorial Boards des International Journal of Shape Modeling, sowie Program Chair von SCCG 2005 (Budmerice, Slowakei).

\section{Institut für Industriemathematik}

Dem Institut angegliedert sind das Kompetenzzentrum Industriemathematik (IMCC) und das Johann Radon Institute for Computational and Applied Mathematics (RICAM)

Raum-, Sach-, Personalausstattung

Der nutzbare Raumbestand des gesamten Instituts mit IMCC und RICAM beträgt $674 \mathrm{~m}^{2}$. Die Ausstattung ist nach Auskunft des Instituts ausgezeichnet. Darüber hinaus gibt es 7 PCs und 6 Notebooks für Studenten und Diplomanden, außerdem Zugang zum Hochleistungsrechner der Universität. Die Bibliothek wird vom Institut als die wichtigste Infrastruktureinrichtung genutzt.

Das Institut verfügt über folgende Finanzmittel

\begin{tabular}{|c|c|c|c|}
\hline in $€$ & 2001 & 2002 & 2003 \\
\hline Laufende Ausgaben & 14.918 & 14.406 & 16.494 \\
\hline Investitionen (Bundesmittel) & 10.123 & 112.009 & 11.047 \\
\hline Investitionen (Drittmittel) & 582.454 & 464.784 & 287.166 \\
\hline davon Personalausgaben & 476.104 & 415.331 & 214.545 \\
\hline davon Sonstige Ausgaben & 106.350 & 49.453 & 72.621 \\
\hline Einnahmen Bundesmittel & 25.041 & 126.415 & 27.541 \\
\hline Drittmittel über die Universität & 340.524 & 235.066 & 125.094 \\
\hline Drittmittel FWF & 285.940 & 288.894 & 145.927 \\
\hline $\begin{array}{l}\text { Sonstige Drittmittel, die organi- } \\
\text { satorisch nicht über die Uni lau- } \\
\text { fen, aber dem Institut zuzuordnen } \\
\text { sind wie CD-Labors, Kompe- } \\
\text { tenzzentren (bitte unten erläutern) }\end{array}$ & & 953.200 & 1.115 .000 \\
\hline
\end{tabular}

Es gibt am Institut aus Haushaltsmitteln finanzierte 5,5 Stellen und aus Sonderprogrammen 16 Stellen auf Zeit. Des weiteren gibt es 4 drittmittelfinanzierte Stellen auf Zeit, die seit 1.4.2004 über das SFB-Projekt F1308 am Johann Radon Institute for Computational and Ap- 
plied Mathematics (RICAM) der ÖAW angesiedelt und finanziert sind; die Tätigkeit der vom FWF finanzierten MitarbeiterInnen, die im Berichtszeitraum am Institut für Industriemathematik angestellt waren, ist in diesem Bericht enthalten.

Von den 16 Drittmittelstellen ist nur eine am Institut für Industriemathematik angesiedelt, 15 Stellen sind am Kompetenzzentrum Industriemathematik (IMCC), das bis Mitte 2002 organisatorisch am Institut war und seither ausgelagert ist.

Zusätzlich besteht seit 2003 das ÖAW- Institut RICAM, dessen Direktor der Institutsdirektor in Personalunion ist; es ist derzeit im Aufbau, verfügt zum 1.7. über 16 Post Doc- und 7 Doktorandenstellen, davon 14 aus ÖAW- Mitteln und 9 aus Drittmitteln. Die Ausbaupläne gehen von 27-30 Post Doc- und mindestens derselben Zahl von Doktorandenstellen (letztere aus Drittmitteln) aus. Das RICAM verfügt über 6 Abteilungen, alle am Standort Linz, geleitet von den Professoren der Universität Linz, Wien und Graz.

\section{Besondere Aktivitäten in der Lehre}

Doktoranden betreuen, soweit es nicht mit Ihrer Finanzierung (Industrieprojekte) im Widerspruch steht, Übungen und Praktika, Habilitanden halten selbständige Vorlesungen, wie auch alle promovierten Universitäts-Assistenten). Es gibt einen europäischen Graduiertenkurs „Mathematics for Industry“.

\section{Publikationen}

Die Publikationen des Instituts umfassen 37 referierte Artikel in Zeitschriften, 7 in Tagungsbänden und Sammelwerken und 24 in Preprintserien, gehaltene Vorträge 67 (im Zeitraum 2001-2003). Von 2000 bis 2003 wurden 5 Patente erteilt.

\section{Wissenschaftlicher Nachwuchs}

Von 1999 bis 2003 wurde 1 Habilitation im Zeitrahmen von 7 Jahren abgeschlossen. Es gab 10 Promotionen, 4 davon industriefinanziert.

\section{Personalfluktuation}

In den letzten 5 Jahren haben 16 Wissenschaftler das Institut verlassen, davon sind 9 Abgänge in die Industrie, 4 auswärtige Berufungen und 3 auf andere Universitätsassistentenstellen zu verzeichnen. Demgegenüber stehen 20 Neuzugänge. Das Gewinnen und Halten von Mitarbeitern ist aufgrund des hohen Drittmittelaufkommens problemlos. 


\section{Kooperation in der Forschung}

Aus dem Institut ist eine Spin-off- Firma hervorgegangen: MathConsult GmbH. Das Institut arbeitet im Rahmen von EU-Netzwerken mit verschiedenen internationalen Universitäten und anderen Forschungseinrichtungen zusammen (z.B. TU Eindhoven, Universität Kaiserslautern, Universitá degli Studi di Milano, FhG zur Förderung der angewandten Forschung, General Electrics Medical Systems, Henkel KGaA).

\section{Anerkennung der Leistungen des Fachbereichs}

Einige Mitglieder des Instituts waren in verschiedener Funktion als Gutachter und Kommissionsmitglieder in In- und Ausland tätig. Eine Reihe wichtiger nationaler und internationaler Ehrungen ging an Mitglieder des Instituts.

Zwischen 2002 und 2004 gab es 8 Auslandsaufenthalte (Gastprofessuren) von 5 Institutsmitgliedern. Die Hälfte der Auslandsaufenthalte betrug 1 Jahr.

\section{Institut für Numerische Mathematik}

\section{Raum-, Sach-, Personalausstattung}

Das Institut beurteilt die räumliche Ausstattung als ausreichend. Es stehen auf 245,40 $\mathrm{m}^{2} 12$ Büroräume zur Verfügung. Pro wiss. Mitarbeiter gibt es 1 PC und 1 Laptop. Es steht ein PCLab mit 13 Dualprozessoren zur Verfügung. Der Zugriff auf ALTIX des ZID ist gegeben.

Das Institut verfügt über folgende Finanzmittel

\begin{tabular}{|l|r|r|r|}
\hline in $€$ & 2001 & 2002 & 2003 \\
\hline Laufende Ausgaben & $9.011,43$ & $13.132,00$ & $11.818,00$ \\
\hline Investitionen (Bundesmittel) & $44.206,89$ & $28.330,02$ & $19.828,75$ \\
\hline Einnahmen Bundesmittel & 315,77 & 478,10 & 758,90 \\
\hline Drittmittel über die Universität & $7.921,34$ & $2.200,00$ & $15.500,00$ \\
Drittmittel FWF & $224.506,50$ & $324.506,50$ & $383.082,98$ \\
\hline
\end{tabular}

Das Institut arbeitet in 8 Sonderforschungsbereichen und Industrieprojekten, die über Drittmittel finanziert sind.

Die personelle Ausstattung wird vom Institut als gut beurteilt. Es gibt 7 Stellen, die aus Haushaltsmitteln finanziert sind und 11 Stellen auf Zeit über Drittmittel. 


\section{Besondere Aktivitäten in der Lehre}

Doktoranden und Habilitanden werden durch Lehraufträge zwischen 2 und 4 SWS in die Lehre eingebunden. Die Nachwuchswissenschaftler werden durch Projektseminare, Methodenseminare, Diplomanden- und Dissertantenstunden auf ihre Aufgaben in der Lehre vorbereitet.

\section{Publikationen}

Am Institut gab es im Zeitraum von 2001-2003 folgende Publikationstätigkeiten:

6 Monographien, 39 Aufsätze in Zeitschriften, davon 29 referiert und 23 Beiträge zu Sammelwerken. Es wurden 105 Vorträge gehalten, davon 38 auf Einladung.

\section{Wissenschaftlicher Nachwuchs}

Im Zeitraum 1999-2003 gab es 4 Promotionen mit einer durchschnittlichen Promotionsdauer von 39,75 Monaten. Das Durchschnittsalter lag bei 28,25 Jahren. In den letzten 5 Jahren gab es nur 1 Habilitation, deren Dauer 10 Jahre betrug. Der Habilitand war zum Zeitpunkt der Habilitation 38 Jahre alt.

\section{Personalfluktuation}

5 Weggängen steht 1 Neuzugang gegenüber. Das Institut sieht die Schwierigkeit, qualifiziertes Personal zu gewinnen, teilweise in der Abwerbung der Industrie auf gutbezahlte Stellen.

\section{Kooperationen in der Forschung}

Das Institut arbeitet mit 15 ausländischen Kooperationspartner aus Universitäten und Industrie zusammen ( z.B. Bosch GmbH, Deutschland, Technischen Universität München). Aus der Forschung hervorgegangen ist eine Spin-off Firma: FEMworks Finite Elemente Software und Consulting GmbH.

\section{Institut für Stochastik}

\section{Raum-, Sach-, Personalausstattung}

Die technische Ausstattung besteht aus zum Teil veralteten Computern. Jeder Mitarbeiter verfügt über ein eigenes Büro.

Das Institut verfügt über folgende Finanzmittel

\begin{tabular}{|l|r|r|r|}
\hline in $€$ & 2001 & 2002 & 2003 \\
\hline laufende Ausgaben & 6318 & 6644 & 5811 \\
\hline Investitionen (Bundesmittel) & 7710 & 7700 & 5201 \\
\hline Einnahmen (Bundesmittel ) & 14028 & 14344 & 11012 \\
\hline
\end{tabular}


Am Institut gibt es insgesamt 4,75 Stellen, die aus Haushaltsmitteln finanziert sind. Die Personalressourcen werden von den vielen Servicelehrveranstaltungen, die vom Institut abgehalten werden, stark beansprucht. Zusätzlich werden durch die Mitgliedschaft in zahlreichen Kollegialorganen und Unigremien weitere Personal-Ressourcen gebunden. Die räumliche Ausstattung ist gut.

\section{Besondere Aktivitäten in der Lehre}

Doktoranden führen Übungen zu LVA für Mathematiker und Mechatroniker durch.

\section{Publikationen}

Im Zeitraum 2001-2003 gab es 5 referierte Aufsätze, davon 4 in Zeitschriften. Es wurden 5 Vorträge gehalten.

\section{Wissenschaftlicher Nachwuchs}

In den letzten 5 Jahren gab es weder Promotionen noch Habilitationen.

\section{Personalfluktuation}

In den letzten 5 Jahren gab es 3 Abgänge, davon 1 Ruf an eine Fachhochschule. Demgegenüber stehen 2 Neuzugänge. Das Institut sieht es als sehr schwierig an, qualifizierte Mitarbeiter zu gewinnen, was zu freien Stellen führt, die vorübergehend nicht besetzt werden können.

\section{Kooperation in der Forschung}

Ein Institutsmitglied war als Referee für Zeitschriften tätig und Mitglied des Scientific Board von Konferenzen. Das Institut arbeitet beim Studentenaustausch und bei Diplomarbeiten mit Industriepartnern zusammen.

\section{Institut für Symbolisches Rechnen}

\section{Raum-, Sach-, Personalausstattung}

An Geräten sind vorhanden: 30 Linux- PCs (15 mit P4, 15 mit P3 CPU), 25 Linux- Laptops (10 mit P4, 15 mit P3 CPU), 2 SGI, 1 Sun Workstation, 3 Abteilungsdrucker, 1 Beamer.

Das Institut verfügt über folgende Finanzmittel

\begin{tabular}{|l|r|r|r|}
\hline in $€$ & 2001 & 2002 & 2003 \\
\hline laufende Ausgaben [o.Dot] & $29.280,00$ & $33.964,00$ & $30.000,00$ \\
\hline Investitionen (Bundesmittel) [a.o.Dot] & $50.363,37$ & $42.755,12$ & $7.593,00$ \\
\hline $\begin{array}{l}\text { Drittmittel über die Universität (bzw. } \\
\text { FWF): gesamte genehmigte Drittmit- } \\
\text { tel inkl. Personalkosten }\end{array}$ & $403.941,37$ & $541.757,94$ & $580.447,88$ \\
\hline
\end{tabular}


Am Institut gibt es 12 Stellen, die aus Haushaltsmitteln finanziert werden, 20 Stellen finanziert aus Sonderprogrammen (davon 10 auf Zeit) sowie 20 Stellen drittmittelfinanziert (davon ebenfalls 10 auf Zeit). Eine Professur ist seit 1.11.2002 unbesetzt, die Berufungsverhandlungen laufen.

Nach Ansicht des Institutes wird die räumliche Grundausstattung als durchaus befriedigend beurteilt. Im Campus steht eine Nutzfläche von insgesamt $46 \mathrm{~m}^{2}$ für 3 Räume zur Verfügung. Hinzu kommen $560 \mathrm{~m}^{2}$ im Schloss Hagenberg, verteilt auf 20 Räume. Es gibt keine Großgeräte, die Mitarbeiter sind mit Arbeitsplatzrechnern ausgerüstet.

\section{Besondere Aktivitäten in der Lehre}

Doktoranden halten generell keine selbständigen Lehrveranstaltungen ab, sind aber von Fall zu Fall als Übungsleiter eingesetzt; Mitarbeiter mit Doktorat bzw. Habilitanden halten Lehrveranstaltungen im Ausmaß von etwa 4 Wochenstunden; es gibt am Institut eine interne Evaluation von Lehrveranstaltungen.

RISC führt seit vielen Jahren ein geregeltes Doktoratsstudium in Symbolic Computation durch, mit durchschnittlich etwa 25 Doktoranden. Das Doktoratsstudium wird jährlich international ausgeschrieben, derzeit wird es von 24 Teilnehmern aus 14 Ländern absolviert. Das Doktoratsstudium ist auf 4 Jahre angelegt, wobei das erste Jahr dem Besuch von Vorlesungen aus Symbolic Computation und der Verfassung eines Proposals gewidmet ist, das zweite und dritte Jahr der Forschungsarbeit, und das vierte Jahr der Verfassung der Dissertation und der Präsentation der Ergebnisse. Vorlesungen und Seminare werden auf Englisch abgehalten.

\section{Publikationen}

Im Zeitraum 2001-2003 gab es 11 editierte Publikationen in Sammelwerken und Proceedings, 29 referierte Aufsätze in Zeitschriften, 87 referierte Beiträge in Sammelwerken und Proceedings. Es wurden 130 Vorträge gehalten, davon 69 auf Einladung.

\section{Wissenschaftlicher Nachwuchs}

Im Zeitraum 1999-2003 gab es 19 Promotionen mit durchschnittlicher Dauer von 4,7 Jahren. Das Durchschnittsalter lag bei 30 Jahren. Die Zahl der Habilitationen lag bei 3 mit einer Dauer von 6,3 Jahren. Das Durchschnittsalter lag hier bei 39 Jahren. 


\section{Personalfluktuation}

Das Institut sieht die Schwierigkeit bei Berufungen in der angespannten Finanzlage der Universität und der gleichzeitigen Anstellung auf Vertragsbasis.

\section{Kooperation in der Forschung}

Es wurde eine Spin-off- Firma gegründet: RISC Software GmbH. Das Institut unterhält vielfältige Kooperationsbeziehungen zu Universitäten und Instituten im Ausland (z.B. Universidad de Alcala de Henares, Madrid, Universität Debrecen, Ungarn). Die Aktivitäten dienten der Integration der Forschungsgebiete Computer-Algebra und Automatisches Beweisen und der Entwicklung von innovativen Software-Werkzeugen für eine neue Art von mathematischen Software-Systemen, die alle Stufen im mathematischen Explorationsprozess unterstützen.

\section{Anerkennung der Leistungen des Fachbereichs}

Die Mitglieder des Institutes waren in verschiedener Funktion als Gutachter und Mitorganisatoren von Konferenzen und Tagungen im In- und Ausland tätig. Ein Institutsmitglied erhielt Preise für sein wissenschaftliches Gesamtwerk.

\section{Institut für Wissensbasierte Mathematische Systeme}

Raum-, Sach-, Personalausstattung

Das Institut hat leistungsfähige Arbeitsplatzrechner vor allem für Bildverarbeitung und Datenanalyse, Drucker, Scanner, Online-Zugang und Vorortverfügbarkeit von diversen Journalen.

Das Institut verfügt über folgende Finanzmittel

\begin{tabular}{|l|r|r|r|}
\hline in $€$ & 2001 & 2002 & 2003 \\
\hline laufende Ausgaben & $6.303,68$ & $5.739,03$ & $7.324,42$ \\
\hline Investitionen (Bundesmittel) & $3.851,74$ & $6.690,18$ & $5.357,56$ \\
Investitionen (Drittmittel) & $15.135,00$ & $11.299,80$ & $10.648,43$ \\
\hline Einnahmen Bundesmittel & $10.922,96$ & $12.827,00$ & $11.778,00$ \\
\hline Drittmittel über die Universität & $406.508,75$ & $541.766,59$ & $393.282,67$ \\
\hline
\end{tabular}

Das Institut beurteilt seine Ausstattung als bedenklich wegen des hohen Drittmittelanteils insbesondere bei der Finanzierung von Räumen, Technik und Personal. Es verfügt an der Universität Linz über 3 Räume $\left(50 \mathrm{~m}^{2}\right)$ mit 5 Computerarbeitsplätzen. Darüber hinaus stehen im Fuzzy Logic- Laboratorium Hagenberg ein Forschungszentrum mit $180 \mathrm{~m}^{2}$ und $10 \mathrm{Compu}-$ 
terarbeitsplätzen zur Verfügung. Das Institut verfügt insgesamt über 12,5 Stellen. Davon werden über Haushaltsmittel rund 5 Stellen (2 Professuren), aus Sonderprogrammen rund 4 Stellen und über Drittmittel rund 4 Stellen finanziert. Das Institut bemängelt eine Unterdotierung im Bereich der wissenschaftlichen MitarbeiterInnen, insbesondere für die Grundlagenforschung und für die Lehre steht nur ein Mitarbeiter zur Verfügung, der ausschließlich die wissenschaftliche Leitung des Software Competence Centers Hagenberg wahrnimmt.

\section{Besondere Aktivitäten in der Lehre}

MitarbeiterInnen auf Universitätsstellen werden extensiv in der Lehre eingesetzt aufgrund der hohen Anzahl an Service-Lehrveranstaltungen des Instituts; externe Nachwuchswissenschaftler dagegen nach Maßgabe der finanziellen Möglichkeiten und des persönlichen Zeitbudgets. Es besteht ein 2-jähriges internes Curriculum zum Themenbereich Wissensbasierte Mathematische Systeme. Diplomanden- und Dissertantenseminare werden in Form von kleinen Tagungen organisiert.

\section{Publikationen}

Von 2001 bis 2003 wurden insgesamt 60 Publikationen verfasst (darunter 19 in referierten Zeitschriften, 30 in referierten Tagungsbänden und Sammelwerken). Es wurden 47 Vorträge gehalten, davon 6 auf Einladung.

\section{Wissenschaftlicher Nachwuchs}

Von 1999 bis 2003 gab es 6 Promotionen mit einer durchschnittlichen Dauer von 5,3 Jahren. Das Durchschnittsalter lag bei 30,5 Jahren. Im gleichen Zeitraum gab es 1 Habilitation mit einer Dauer von 5 Jahren. Der Habilitand war 31 Jahre.

\section{Personalfluktuation}

7 Wissenschaftler haben in den letzten 5 Jahren den Fachbereich verlassen, dem gegenüber stehen 9 Neuzugänge.

\section{Kooperation in der Forschung}

Das Institut unterhält Kooperationsbeziehungen mit anderen Hochschulen im Ausland (z.B. Slovak University of Technology, Bratislava, University of Haifa, Haifa), mit nationalen Industrieunternehmen (z.B.SONY DADC Austria AG, Anif) und außeruniversitären Einrichtungen (z.B. IKMA- Industrielles Kompetenzzentrum für Mechatronik und Automatisierung). 
Anerkennung der Leistungen des Instituts

Die Mitglieder des Instituts waren in verschiedener Funktion als Gutachter und Herausgeber von Zeitschriften im In- und Ausland tätig. Eine Reihe wichtiger nationaler und internationaler Preise ging an Wissenschaftler aus dem Institut. 


\section{Teil B: Lehre und Studium am Fachbereich Mathematik der Universität Linz}

\section{Ausbildungsprofil}

Die Universität Linz bietet als einzige österreichische Universität Technische Mathematik als Bakkalaureat- Magisterstudium an, das im Rahmen des Bologna Prozesses ab dem WS 2003/2004 eingeführt wurde.

Die innerhalb Österreichs für Linz spezifischen Schwerpunktsetzungen sind die Magisterstudien Industriemathematik und Computermathematik.

\section{Allgemeine Ziele}

Ziel des Bakkalaureatstudiums ist es, über eine breite Ausbildung in den mathematischen Grundlagen die Studierenden mit den wichtigsten Theorien und Methoden der Technischen Mathematik vertraut zu machen. Insbesondere sollen die Studierenden befähigt werden, die Theorien und Methoden auf reale Problemstellungen anzuwenden. Die AbsolventInnen des Bakkalaureatstudiums sollen die Fähigkeit besitzen, bekannte mathematische Methoden und bestehende Computersoftware einzusetzen und erforderlichenfalls diese Methoden und Programme geeignet basierend auf einem tiefgehenden Verständnis der mathematischen Grundlagen nach dem aktuellen Stand der Wissenschaft zu adaptieren und weiterzuentwickeln.

Ziel der Magisterstudien ist es, dieses Wissen zu vertiefen durch Schwerpunktsetzungen und die Studierenden in einer für das jeweilige Magisterstudium charakteristischen Fächergruppe an den aktuellen Stand der Wissenschaft heranzuführen. Die AbsolventInnen der Magisterstudien sollen auch in der Lage sein, neue Methoden zu entwickeln und in der Praxis einzusetzen, basierend auf aktuellen Forschungsergebnissen. Die Studien befähigen zu eigenständiger fachlicher Weiterbildung und fördern die Fähigkeit zu Kommunikation und Zusammenarbeit im Team, auch mit Fachleuten des jeweiligen Anwendungsgebietes.

\section{Praxisbezug}

Die Universität bietet ihren AbsolventInnen Kontakte zu potentiellen Einstellungsträgern aus Industrie und Wirtschaft an. Diese Kontakte bestehen über Kooperationen der mathematischen Institute und über Spin-off- Firmen und Kompetenzzentren des Fachbereichs. Der Praxisbezug wird in Projektseminaren vermittelt, deren Inhalte oft von den Industriepartnern angeregt wurden. Viele der Lehrenden des Fachbereichs haben direkte Erfahrungen mit anwendungsbezogenen Projekten und setzen dieses Wissen in den Lehrveranstaltungen um. 
Forschungsschwerpunkte, wie zum Beispiel die des Spezialforschungsbereichs „Numerical and Symbolic Computation“ stehen im engen Zusammenhang mit Ausbildungsschwerpunkten wie Industriemathematik und Computermathematik.

Über die internationalen Programme ERASMUS und CEEPUS werden den Studenten Auslandssemester und -erfahrungen ermöglicht, was auch rege in Anspruch genommen wird. Darüber hinaus gibt es Planungen eines gemeinsamen internationalen Master-Programms mit Kaiserslautern und Eindhoven.

\section{Personelle Ressourcen}

Die Lehrkapazität des ständigen Lehrkörpers (9 Professoren, 13 Dozenten, 17 Assistenten und 21 externe Lektoren) müsste 278 SWS betragen.

Das tatsächliche Lehrangebot hat diese Kapazität in den letzten 5 Jahren nicht erreicht, es kommt im SS04 auf eine SWS von 231.

Der Fachbereich Mathematik bietet Service- Lehrveranstaltungen für die Studienrichtungen Physik, Chemie, Informatik, Mechatronik und Wirtschaftswissenschaften.

Das sind die Service-Lehrveranstaltungen:

\begin{tabular}{|l|r|r|}
\hline & WS 03 & SS 04 \\
\hline Lehramt Mathematik & 4 & 10 \\
\hline Studiengänge anderer Institute & 79 & 54 \\
\hline
\end{tabular}

Die Lehrveranstaltungen Analysis und Lineare Algebra (insgesamt 28 SWS) werden auch von den Studierenden der Studienrichtung Technische Physik besucht. Viele der Pflicht- und Wahlfächer des Studiums der Technischen Mathematik können auch von Studierenden des Lehramts Mathematik als Pflicht- bzw. Wahlfächer belegt werden.

\section{Sachausstattung}

Es stehen 27 Computerarbeitsplätze (PC + Drucker, 1 Beamer) den Studierenden rund um die Uhr zur Verfügung. 7 Laptops für die Studierenden des 1. Jahres und 3 Laptops für Diplomanden werden leihweise zur Verfügung gestellt.

In der Fachbibliothek stehen in erster Linie Einzelexemplare zur Verfügung. Der Bestand wird laufend aktualisiert. Online- Zugang zu den Mathematical Reviews ist gegeben. Die Hauptbibliothek enthält auch Lehrbuchsammlungen.

Elektronische Dienste für Elektronische Buch- und Zeitschriftensuche, Zentralblatt-Abfragen sind über die Hauptbibliothek verfügbar. 
Der Fachbereich würde sich wünschen, eine noch bessere Ausstattung der Studierenden mit Lizenzen von mathematischer Software wie MATHEMATICA und MATLAB, mehr Arbeitsplätze für Diplomanden und Doktoranden an den Instituten zu erhalten.

Studienanfänger $(w / m)$

\begin{tabular}{|l|r|r|r|r|r|}
\hline $\begin{array}{l}\text { Studiengang/angestrebter Ab- } \\
\text { schluss }\end{array}$ & 1999 & 2000 & 2001 & 2002 & 2003 \\
\hline Technische Mathematik 1 FS & $18 / 61$ & $37 / 67$ & $28 / 51$ & $22 / 74$ & $27 / 47$ \\
$1 \mathrm{HS}$ & $14 / 19$ & $29 / 37$ & $20 / 20$ & $19 / 30$ & $21 / 24$ \\
\hline
\end{tabular}

Die Zahl der Studienanfänger

mit LehramtsstudentInnen beträgt damit in Relation (Vollzeitäquivalente, Durchschnitt der letzten 5 Jahre): 106,2

- je Professorenstelle (9): 11,8

- je Stelle Wissenschaftliches Personal (40): 2,7 sowie

ohne LehramtsstudentInnen beträgt die Zahl der Studienanfänger: (Durchschnitt der letzten 5 Jahre) 86,4 , d.h.

- je Professorenstelle (9) 9,6 und

- je Stelle Wissenschaftliches Personal (40) 2,2.

\section{Studierende $(\mathrm{m} / \mathrm{w})$}

a) nach Studiengängen/angestrebten Abschlüssen und Fachsemestern

\begin{tabular}{|l|r|r|r|r|r|r|r|r|r|r|}
\hline Studiengang & \multicolumn{9}{|l|}{ Studienrende im .......Fachsemester } \\
& 1. & 2. & 3. & 4. & 5. & 6. & 7. & 8. & 9. & 10 \\
\cline { 2 - 11 } & $3 / 9$ & $20 / 19$ & $1 / 13$ & $13 / 41$ & $1 / 12$ & $13 / 19$ & $3 / 6$ & $12 / 25$ & $0 / 12$ & $5 / 21$ \\
\hline Technische Mathematik & 11. & 12. & 13 & 14. & 15. & 16. & 17. & $18 .+$ & & \\
\hline Technische Mathematik & $0 / 2$ & $4 / 13$ & $1 / 1$ & $2 / 6$ & $0 / 1$ & $0 / 1$ & $0 / 0$ & $9 / 26$ & & \\
\hline
\end{tabular}

b) Anteile der Studierenden innerhalb der Regelstudienzeit (nach Studiengängen) $79 \%$

Es befanden sich danach 248 Studierende in der Regelstudienzeit. Auf jede Professorenstelle

(9) kamen 27,6 Studierende, je Stelle wissenschaftliches Personal (40) 6,2 Studierende 


\section{Studien- und Prüfungsorganisation}

Es gibt entsprechende Empfehlungen zur Abfolge der Pflichtlehrveranstaltungen des Bakkalaureatstudiums. Die Abfolge wird durch eine entsprechende Semesterangabe bei der Lehrveranstaltungsliste des jeweiligen Semesters bekannt gegeben. Demnach entfallen auf

- Bakkalaureatstudium: 92 SWS Pflicht-, 12 Wahlpflicht- und 12Wahlveranstaltungen - Von den 92 SWS in den Pflichtfächern entfallen 12 SWS auf Übungen und 38 SWS auf kombinierte Lehrveranstaltungen, die sich aus einem Vorlesungs- und einem Übungsteil zusammensetzen. Die Lehrveranstaltungen Programmierung (3 SWS) und Algorithmische Methoden $(2+2$ SWS $)$ werden zusätzlich durch Tutoren begleitet.

- Magisterstudium Mathematik in den Naturwissenschaften: 22 SWS Pflicht-, 22 Wahlpflicht- und 5 Wahlveranstaltungen

- Magisterstudium Industriemathematik:21 SWS Pflicht-, 23 Wahlpflicht- und 5 Wahlveranstaltungen

- Magisterstudium Computermathematik: 21 SWS Pflicht-, 23 Wahlpflicht- und 5 Wahlveranstaltungen

Der Katalog der Wahlmöglichkeiten im Studienplan umfasst 238 SWS Lehrveranstaltungen an studienplangebundenen Wahlfächern, die in einem zwei- bis vierjährigen Rhythmus angeboten werden. Die Einführungslehrveranstaltungen werden überwiegend von Professoren, zu einem geringeren Anteil von Dozenten abgehalten. Die begleitenden Übungen werden großteils vom Mittelbau bestritten. Die Pflichtlehrveranstaltungen werden regelmäßig abgehalten. Aus Kapazitätsgründen können die meisten Wahlpflichtlehrveranstaltungen nur in einem zwei- bis vierjährigen Rhythmus angeboten werden.

\section{Prüfungsverfahren}

Bisher wurde die 1. Diplomprüfung durch einzelne Prüfungen nach den entsprechenden Lehrveranstaltungen abgelegt. Im neuen Bakkalaureatstudium ist eine Abschlussprüfung verpflichtend (es gibt bisher noch keine AbsolventInnen).

Die 2. Diplomprüfung (im Zukunft die Magisterprüfung) als Abschlussprüfung wird von fast allen KandidatInnen bestanden. Wiederholungen sind extrem selten.

Beim Bakkalaureatstudium sind zwei Bakkalaureatarbeiten im Rahmen von zwei einsemestrigen Lehrveranstaltungen anzufertigen.

Bei der Magisterarbeit muss nach Gesetz sichergestellt sein, dass die Magisterarbeit in 6 Monaten (Vollzeit) fertiggestellt werden kann. Da die Studierenden meist auch parallel Lehrver- 
anstaltungen während der Arbeit an der Diplomarbeit besuchen, ist mit einem Studienjahr zur Fertigstellung der Magisterarbeit zu rechnen.

Die Anmeldung bei Abschlussprüfungen beträgt 4 Wochen vor dem Prüfungsantritt. Die Begutachtung der Magisterarbeit erfolgt kurzfristig nach Einreichung der Arbeit.

Zur Wiederholung von Prüfungen gibt es keine eigenen expliziten Regelungen.

\section{Leistungsanforderungen}

Das Bakkalaureatstudium umfasst 116 SWS, das Magisterstudium 49 SWS. Beide Studien sind innerhalb der vorgesehenen Planstudienzeit studierbar.

Im Bakkalaureatstudium müssen 31 Lehrveranstaltungsprüfungen in den Pflichtlehrveranstaltungen, 8 - 16 weitere Lehrveranstaltungsprüfungen in den Wahlpflicht- und Wahllehrveranstaltungen besucht werden.

Im Magisterstudium müssen 7 - 8 Lehrveranstaltungsprüfungen in den Pflichtlehrveranstaltungen, 9 - 18 weitere Lehrveranstaltungsprüfungen in den Wahlpflicht- und Wahllehrveranstaltungen besucht werden. Das ergibt im Durchschnitt 6- 7 Lehrveranstaltungsprüfungen im Semester.

Es gibt vergleichsweise sehr wenige erwerbstätige Studierende.

\section{Studienerfolg}

Im Zeitraum 1999-2003 wurden von 56 AbsolventInnen 62 Zwischenprüfungen/Vordiplome abgelegt.

Die Fachstudiendauer bis zum Abschluss der Vordiplom- bzw. Zwischenprüfung betrug in den Jahren 1999-2000 durchschnittlich 5 Semester.

\section{Qualitätssicherung}

In der Studienkommission werden bei Bedarf Beratungen über Verbesserungen in der Lehre geführt. Es findet regelmäßiger Erfahrungsaustausch mit KollegInnen des Instituts für Didaktik statt. Die Studienkommission tagt regelmäßig und diskutiert studienrelevanter Fragen. Beim Übertritt von der Schule zur Universität treten die üblichen Schwierigkeiten auf. Das Institut für Didaktik der Mathematik bietet regelmäßig vor Semesterbeginn Vorkurse an und gibt das Feedback darüber an die Institute weiter.

Die didaktische Eignung ist eine der Grundvoraussetzungen für die Habilitation. Die Habilitationskandidaten haben bisher fast ausschließlich bereits Lehrerfahrung vorzuweisen gehabt. Die didaktischen Fähigkeiten werden an Hand dieser Lehrveranstaltungen durch Studenten, die diese Lehrveranstaltungen besucht haben, beurteilt. In einzelnen Instituten werden zu En- 
de des Semesters die Studenten zu den Lehrveranstaltungen befragt. Bei Schwierigkeiten führt der Institutsvorstand ein Gespräch mit den betreffenden Lehrveranstaltungsleitern.

\section{Gesamteinschätzung des Fachbereichs}

Nach Meinung des Fachbereichs lässt das sehr gute Feedback der Absolventen und ihrer Arbeitgeber auf die hohe Qualität der Praxisausbildung der Absolventen schließen.

Die Qualität der Ausbildung in mathematischen Grundlagen und die erstklassige praxisbezogene Ausbildung in angewandter Mathematik wird als sehr hoch bewertet.

Es wurden das Bakkalaureatstudium der Technischen Mathematik und die anschließenden Magisterstudien konzipiert. Diese müssen sich in den nächsten Jahren bewähren. Die Beibehaltung der hohen Qualität ist dabei vorrangiges Ziel. 


\subsection{Universität Salzburg}

\section{Teil A: Forschung, Wissenschaftlicher Nachwuchs}

\section{Fachbereich Mathematik}

\section{Profil}

Das Forschungsprofil des Fachbereichs Mathematik ist geprägt durch die Schwerpunkte "Diskrete und stochastische Geometrie", "Stochastik und Dynamik" sowie "Metrische Zahlentheorie und zahlentheoretische Numerik“.

Die Lehre hat ihr gegenwärtiges Profil in den Jahren 1999 (Mathematik Lehramt) und 2001 (Mathematik) erhalten. Im Jahr 2001 wurden die neuen Mathematik-Studiengänge implementiert und die Einrichtung der neuen Schwerpunkte verwirklicht.

Ein Charakteristikum des Fachbereichs in Salzburg ist die Stochastiknähe eines größeren Teils der vertretenen Forschungsgebiete. Die Stochastiknähe ermöglicht breite mathematische Kommunikation innerhalb des Fachbereichs sowie Synergien in der Lehre, und sie ist eine sehr gute Basis für die Praxisorientierung.

\section{Organisatorische Gliederung}

Im Fachbereich Mathematik gibt es keine organisatorische Untergliederung. Die Ressourcenverteilung wird vom Fachbereichsleiter vorgenommen.

\section{Raum-, Sach-, Personalausstattung}

Nach Ansicht des Fachbereichs sind die Büroräume funktionell und gut ausgestattet. EDV und Internetzugänge werden als hinreichend angesehen. Der Fachbereich verfügt über 30 PCs. Es gibt 25 Büroräume und 3 Archivräume. Die Fakultätsbibliothek befindet sich im gleichen Gebäude. 3 Netzwerkdrucker stehen zur Verfügung. Der Fachbereich verfügt über einen Rechnerübungsraum mit ca. $100 \mathrm{~m}^{2}$ mit 15 PC-Arbeitsplätzen.

An finanziellen Mitteln stehen zur Verfügung:

\begin{tabular}{|l|r|r|r|}
\hline In $€$ & 2001 & 2002 & 2003 \\
\hline Laufende Ausgaben & $17.935,66$ & $20.940,00$ & $24.418,07$ \\
\hline Investitionen ( Bundesmittel) & $10.915,46$ & $27.560,00$ & $12.463,39$ \\
\hline Drittmittel über Universität & $167.058,97$ & $228.826,76$ & $127.573,87$ \\
\hline Sonstige Drittmittel & $12.717,76$ & $26.413,25$ & $45.791,00$ \\
\hline
\end{tabular}


Die Mittel für die Beschaffung von Zeitschriftenabonnements sind nach Ansicht des Fachbereiches stark eingeschränkt worden, die Versorgung erfolgt daher teilweise über die Fernleihe. Es gibt Zugang zu den großen mathematischen Datenbankservern sowie den üblichen kostenlosen Zugang zur allgemeinen Internetnutzung.

Nach Ansicht des Fachbereichs fehlen Büroräume, um alle Wissenschaftler adäquat unterzubringen.

Die personelle Ausstattung wird vom Fachbereich als ungenügend bezeichnet. Es gibt 16 Personalstellen (3 Professoren, 10 wiss. Personal (alle habilitiert), 3 nichtwiss. Personal), die aus Haushaltsmitteln finanziert werden, und 3 Stellen für Forschungsassistenten aus Drittmitteln. Es besteht eine Vakanz zwischen Soll- und Iststand.

\section{Publikationen / Vorträge}

Der Fachbereich hat in den letzten 3 Jahren folgende Publikationsleistungen erbracht: 72 Aufsätze, 46 davon in referierten Zeitschriften und Tagungsbänden. Es wurden 69 Vorträge gehalten.

\section{Wissenschaftlicher Nachwuchs}

Doktoranden und Habilitanden sind seit Jahren sowohl in den Lehrbetrieb als auch in die Forschergruppen eingebunden. Die bisherigen Richtlinien für Dissertationen und Habilitationen haben sich als Instrumente der Qualitätssicherung bewährt.

In den letzten 5 Jahren wurden 9 Promotionen abgelegt mit einer durchschnittlichen Promotionsdauer von 2,8 Jahren. Das Durchschnittsalter lag zwischen 26 Jahren in 1999 und 29 Jahren in 2003. Im gleichen Zeitraum gab es 4 Habilitationen, die in einer durchschnittlichen Zeit von 6 Jahren erstellt wurden. Die Habilitanden waren zwischen 37 Jahren in 2001 und 36,5 Jahren in 2003.

Als Graduiertenstudium wird ein Doktoratsstudium angeboten.

\section{Personalfluktuation}

In den letzten 5 Jahren hat 1 Wissenschaftler den Fachbereich verlassen. Es ist 1 Neuberufung erfolgt. Wegen finanzieller Einschränkungen kann der Studienschwerpunkt Versicherungsmathematik nur mit Mühe aufgebaut werden.

Als ein wichtiger Zukunftsaspekt wird die 2007 anstehende Nachbesetzung einer Professorenstelle gesehen. Durch eine Professur im Bereich Stochastik könnte nach Meinung des Fachbereichs eine wesentliche Verstärkung des Profils in Forschung und Lehre erreicht werden. 
Kooperation in der Forschung

Der Fachbereich unterhält Forschungskooperationen zu Universitäten in der ganzen Welt.

Darüber hinaus bestehen Industriekooperationen (z.B. ISD Software und Systeme, Dortmund) und außeruniversitären Forschungseinrichtungen (z.B. Inst. für Limnologie).

Anerkennung der Leistungen des Fachbereichs

Zwei Mitglieder des Fachbereichs sind als Berater für ein Industrieunternehmen tätig, drei weitere arbeiten innerhalb von nationalen Forschungsgruppen bzw. von europäischen Netzwerken mit.

Öffentlichkeitsarbeit / Frauenförderung

Im Rahmen der "Science Week Austria" präsentiert der Fachbereich einmal pro Jahr ausgewählte Forschungsarbeiten einem breiten Publikum.

Mit der jährlichen Teilnahme am Projekt "FIT - Frauen in die Technik", einer Veranstaltung speziell für Schülerinnen des Bundeslandes Salzburg, wird versucht, die Frauenquote im Magisterstudium zu steigern. 


\section{Teil B: Lehre und Studium am Fachbereich Mathematik der Universität Salz- burg}

Ausgangslage

Die Universität bietet folgende Studiengänge an:

- Diplomstudium Mathematik (10 Semester Grundstudium) mit dem Abschluss Magister/Magistra. (Der Studiengang ist auslaufend)

- Lehramtsstudiengang Mathematik ( 9 Semester Grundstudium ) mit dem Abschluss Magister/Magistra

- Bakkalaureatsstudium Mathematik (6 Semester Grundstudium) mit dem Abschluss Bakkalaureus/Bakkalaurea

- Magisterstudium Mathematik ( 4 Semester Aufbaustudium) mit dem Abschluss Magister/Magistra

- Magisterstudium Angewandte Mathematik (4 Semester Aufbaustudium) mit dem Abschluss Magister/Magistra

- Doktoratsstudium der Naturwissenschaften (4 Semester Aufbaustudium) mit dem Abschluss Doktor/Doktorin

\section{Allgemeine Ziele}

Das auslaufende Diplomstudium Mathematik wird durch das Bakkalaureatstudium Mathematik und zwei darauf aufbauende Magisterstudien ersetzt.

Ziel des Lehramtsstudiums ist die Berufsvorbereitung für die Ausübung des Lehramtes an mittleren und höheren Schulen im Unterrichts Fach Mathematik.

Das Bakkalaureatstudium Mathematik bietet eine schlanke Einführung in Kernbereiche und Denkstrukturen der Mathematik. Diese dient einerseits als Berufvorbereitung für Berufsfelder, für die eine mathematische Grundausbildung erforderlich ist, andererseits als Vorbereitung für eines der beiden Magisterstudien der Mathematik oder eines anderen fachlich in Frage kommenden Magisterstudiums.

Das Magisterstudium Mathematik ermöglicht die Berufsvorbereitung und wissenschaftliche Vertiefung in unterschiedlichen Teilgebieten der Mathematik, wobei durch die Einrichtung von Schwerpunktfächern Spezialisierungen vorgenommen werden können.

Das Magisterstudium Angewandte Mathematik ermöglicht die Berufsvorbereitung und wissenschaftliche Vertiefung in einem der Schwerpunktfächer Dynamische System, Finanz- und Versicherungsmathematik und Statistik. 
Das Studium zum Erwerb des Doktorats der Naturwissenschaften dient über die wissenschaftliche Berufsvorbereitung hinaus der Weiterentwicklung der Befähigung zu selbständiger wissenschaftlicher Arbeit sowie der Heranbildung und Förderung des wissenschaftlichen Nachwuchses.

Die Studienziele sind in den jeweiligen Studienplänen dokumentiert. In der Studienkommission Mathematik wurden nach einem institutsweiten Diskussionsprozess Inhaltsvereinbarungen sowohl für eine Reihe von aufbauenden Lehrveranstaltungen als auch für die kommissionelle Bakkalaureatprüfung getroffen.

\section{Praxisbezug}

Bis zur Neugestaltung der Curricula im Jahr 2001 hat es Defizite im Anwendungsbezug des Lehrangebotes gegeben. Parallel zu einer erfolgreichen Neuberufung (2001, Geometrie und Versicherungsmathematik) ist eine stärkere Verankerung von praxisbezogenen Lehrveranstaltungen in den Curricula des Bakkalaureatstudiums und des Magisterstudiums durchgeführt worden.

In den Bereichen Versicherungs- und Finanzmathematik und Schulmathematik werden Lehrveranstaltungen von Gastprofessoren mit großer praktischer Erfahrung abgehalten, die im erst genannten Bereich über die Aktuarvereinigung Österreichs und der Österreichischen Gesellschaft für Versicherungsfachwissen unterstützt werden. Diese Veranstaltungen werden von den Studierenden sehr rege genutzt.

Die Universität Salzburg war die erste Universität in Österreich, die im Fach Mathematik das dreistufige Bildungssystem realisiert hat.

Ein internationales Programm in der versicherungsmathematischen Ausbildung ist im Aufbau begriffen.

\section{Personelle Ressourcen}

Die Lehrkapazität des festangestellten Lehrkörpers (3 Professoren, 10 Dozenten) umfasst nach offiziellen Richtwerten 84 SWS. Die tatsächlich geleistete Lehrkapazität betrug im WS 200486 SWS, im SS 2003102 SWS.

An Serviceleistungen für andere Studiengänge wurden vom Fachbereich 21 SWS erbracht.

\section{Sachausstattung}

Die Kapazität der Arbeitsräume ist nach Ansicht des Fachbereichs für die weitere Entwicklung zu gering. Ein Rechnerübungsraum wird gemeinsam mit dem interfakultären Fachbereich Erziehungswissenschaften, Fachdidaktik und LehrerInnenbildung genutzt. Die Ausstattung der Bibliothek mit Standardtiteln wird als zufriedenstellend eingeschätzt. Die 
Aktualisierung des Bestandes leidet jedoch unter der zunehmenden finanziellen Enge. Bei den Zeitschriften reicht der Bestand bereits für Diplomarbeiten und Dissertationen nicht aus. Der Fachbereich behilft sich mit Fernleihe. Der Zugang zu den meisten elektronischen Zeitschriftenabonnements kann aus Kostengründen nicht wahrgenommen werden.

Auf Grund der Österreich-weiten Lizenzen für den Zugang zu den Mathematical Reviews und dem Zentralblatt für Mathematik verfügt der Fachbereich über die grundlegenden und wichtigsten Datenbanken für wissenschaftliche Arbeit. Die EDV-Ausstattung wird als ausreichend betrachtet. Es fehlen noch einige neue Medien, wie ein Laptop für die Lehre und moderne Overhead-Geräte. Auch ein "Medienkoffer Mathematik" (mit populärwissenschaftlichen Büchern, Filmen, etc.) zum Einsatz in der Lehre, sowohl an der Universität als auch in Schulen, ist geplant.

\section{Studienanfänger $(w / m)$}

Die Zahl der Studienanfänger in den letzten 5 Jahren:

1999: 82

2000: 118

2001: 81

2002: 110

2003: 111

\section{Studienanfänger nach angestrebten Abschlüssen (w/m)}

Die Anzahl der Studienanfänger betrug in den einzelnen Studiengängen in den letzten 5 Jahren durchschnittlich:

- Diplomstudium Mathematik (nach Studienplan 1995) : 47

- Lehramtsstudium Mathematik (nach Studienplan 1995): 8

- Lehramtsstudium Mathematik (nach Studienplan 1999): 242

- Diplomstudium Mathematik (nach Studienplan 2000): 40

- Bakkalaureat Mathematik (nach Studienplan 2001): 147

- Magisterstudium Mathematik (nach Studienplan 2001): 11

- Magisterstudium Angewandte Mathematik (nach Studienplan 2001): 6

\section{Studien- und Prüfungsordnung}

Die Abfolge der Vorlesungen ist in den jeweiligen Studienplänen festgeschrieben. Die Vorlesungen des Grundstudiums werden durch Übungsgruppen begleitet. Zur Vorlesung Diskrete Mathematik, welche für vier verschiedene Studien verpflichtend ist, finden bis zu 7 Parallel- 
gruppen statt, da aufgrund des Computereinsatzes die Gruppenstärke auf 25 Personen begrenzt ist.

Die Vorlesungen Analysis I und II, Diskrete Mathematik und Lineare Algebra werden durch Konversatorien begleitet, die schwierige Passagen der Vorlesung nochmals erläutern. Die Lehrveranstaltungen Mathematische Software, Geometrie, Stochastische Modellbildung und Projektpraktikum werden in der Regel durch Tutorien begleitet. Tutorien werden durch Diplomanden und Dissertanten geleitet, die sich in wöchentlichen Sitzungen mit dem Leiter der Vorlesung koordinieren.

In den Übungen zu den Hauptlehrveranstaltungen gib es üblicherweise mehrere Gruppen, die speziell auf bestimmte Teilnehmerprofile (z.B. Lehramt, Bakkalaureat, etc.) ausgerichtet sind. In den Hauptlehrveranstaltungen selbst wechseln die Vortragenden in der Regel jährlich. Bezüglich der Einführungsveranstaltungen werden erfahrene Kollegen leitend eingebunden und mit der Koordination der Parallelgruppen für die Übungen beauftragt. Die Planung des Lehrangebotes erfolgt jeweils für ein Studienjahr.

Bisher wurden alle Pflichtfächer regelmäßig angeboten, einzelne Lehrveranstaltungen im Zwei-Jahres-Rhythmus. Eine ausreichende Wahlmöglichkeit im Bereich der Wahlpflichtfächer konnte ebenfalls gewährleistet werden.

Im Rahmen von Universitätspartnerschaften und mit Hilfe des Socrates-/ErasmusAustauschprogramms werden den Studierenden Auslandsaufenthalte ermöglicht.

\section{Prüfungsverfahren}

Prüfungswiederholungen sind bei Abschlussprüfungen bisher nur in seltenen Fällen vorgekommen. Die Zahl der schriftlichen Vorlesungsprüfungen war 7 (ein Termin am Ende des betreffenden Semesters und je 3 Termine im Laufe der beiden folgenden Semester). Gemäß den neuen gesetzlichen Bestimmungen wird es in Zukunft im Regelfall lediglich 3-4 Prüfungstermine geben. Die mündlichen Prüfungstermine werden zwischen Studierenden und Lehrenden individuell vereinbart.

Das Bakkalaureatstudium sieht 2 Arbeiten vor, die im Rahmen der Lehrveranstaltungen SE (2 SWS) Mathematisches Seminar und PJ (5 SWS) Projektpraktikum abzufassen sind. Insbesondere die zweite Bakkalaureatarbeit erfordert einen beträchtlichen Arbeitsaufwand und ist mit einer Abschlussarbeit zu vergleichen.

Im Diplom- bzw. Magisterstudium wird die Aufgabenstellung der Abschlussarbeiten so gewählt, dass die Bearbeitung innerhalb von 6 Monaten möglich und zumutbar ist, wobei davon ausgegangen wird, dass zu Beginn der Diplomarbeit alle Lehrveranstaltungsprüfungen abgelegt sind. 
Für die Bewältigung des Doktoratsstudiums werden 2 Jahre vorgegeben, wobei Lehrveranstaltungen von 12 SWS zu absolvieren sind. Die Anzahl der Dissertationen lag im Fachgebiet Mathematik bei 6 und in der Fachdidaktik bei 1 Arbeit im Berichtszeitraum.

Hinsichtlich der Wahl der Prüfungsfächer, der Prüfer und des Termins der Prüfung wird nach Möglichkeit dem Wunsch der Studierenden entsprochen. Zwischen Bekanntgabe des Prüfungstermins und der Realisierung der Prüfung müssen laut Prüfungsordnung 3 Wochen liegen.

Für Diplom- und Magisterarbeiten beträgt die gesetzliche Begutachtungsfrist 2 Monate, die jedoch meist weit unterschritten wird. Die gesetzliche Begutachtungsfrist für Dissertationen liegt bei 4 Monaten, sie wird ebenfalls weit unterschritten.

\section{Leistungsanforderungen}

Das Bakkalaureatstudium umfasst 85 SWS. Über die Lehrveranstaltungen sind Einzelprüfungen abzulegen. Im Rahmen von 2 Lehrveranstaltungen sind Bakkalaureatsarbeiten anzufertigen. Die Bakkalaureatsprüfung wird vor einer Kommission abgelegt. Das Studium ist so konzipiert, dass es am Ende des 6. Semesters abgeschlossen werden kann.

Die beiden Magisterstudien haben einen Umfang von 35 SWS. Es sind Einzelprüfungszeugnisse über die Lehrveranstaltungen vorzulegen. Darüber hinaus ist eine Magisterarbeit anzufertigen. Die Magisterprüfung wird vor einer Kommission abgelegt. Das Studium ist so angelegt, dass das Studium am Ende des 4. Semesters abgeschlossen sein kann.

Das Lehramtsstudium umfasst 95 SWS im Fach Mathematik und 70-120 SWS im zweiten Studienfach. Über alle Lehrveranstaltungen sind Einzelprüfungen abzulegen. Darüber hinaus ist der Nachweis einer schulpraktischen Ausbildung zu erbringen. Es ist eine Diplomarbeit aus einem der beiden Unterrichtsfächer zu schreiben. Die Diplomprüfung wird vor einer Kommission abgelegt. Das Studium ist so konzipiert, dass es am Ende des 9. Semesters abgeschlossen werden kann.

\section{Prüfungserfolg}

Die Zahl der AbsolventInnen in den letzten 5 Jahren:

- Diplomstudium Mathematik (Studienplan 1995): 27

- Lehramtsstudium Mathematik (Studienplan 1995): 92

- Lehramtsstudium Mathematik (Studienplan 1999): 44

- Bakkalaureatsstudium Mathematik (Studienplan 2001): 14

- Magisterstudium Mathematik (Studienplan 2001): 1 
(Für das Magisterstudium Angewandte Mathematik und das Diplomstudium Mathematik liegen noch keine Zahlen vor).

Die Chancen der AbsolventInnen auf dem Arbeitsmarkt sind nach den Erfahrungen des Fachbereichs sehr hoch. Lediglich bei den AbsolventInnen des Lehramtsstudiums muss mit Wartezeiten bis zum Stellenantritt gerechnet werden.

\section{Qualitätssicherung}

Die Universität Salzburg hat vor drei Jahren mit einer offiziellen, verpflichtenden Lehrevaluation begonnen. Es werden in regelmäßigem Abstand alle Lehrveranstaltungen evaluiert. Das zentrale Gremium zur Überwachung und Verbesserung der Qualität der Lehre am Fachbereich ist die Curricularkommission. Der Vorsitzende der Studienkommission ist traditionell für die Qualitätskontrolle und die Koordination der Lehrveranstaltungsinhalte zuständig. Da die Computer-Vorkenntnisse der StudienanfängerInnen sehr unterschiedlich sind, hat der Fachbereich die Lehrveranstaltung Einführung in Betriebssysteme und Anwendungen für Personalcomputer eingerichtet, die die Studienanfänger im Rahmen des freien Wahlfaches absolvieren können.

Als Resultat auf die Studienabbrüche in den sehr hohen Semestern hat die Universität das Bakkalaureatstudium eingeführt. Eine Eingangswerkstatt wurde eingerichtet, die den Übergang von der Schule zur Universität zu bewältigen hilft und die Studierenden in das Lesen, Verstehen und die Abfassung mathematischer Texte einführt.

Die didaktische Eignung ist eine der Grundvoraussetzungen für die Habilitation. Die Habilitationskandidaten haben bisher ausschließlich bereits Lehrerfahrung vorzuweisen gehabt. Die didaktischen Fähigkeiten werden an Hand dieser Lehrveranstaltungen durch Studenten, die diese Lehrveranstaltungen besucht haben, beurteilt.

\section{Gesamteinschätzung des Fachbereichs}

Die Stärken und Schwächen der Ausbildung werden vom Fachbereich wie folgt beurteilt: Stärken

- Grundlage des gut funktionierenden Lehrbetriebs ist die gute Gesprächskultur innerhalb des Fachbereiches

- die frühe Einführung des Bakkalaureats- und Magisterstudiums

- der intensiven Betreuung von Diplomarbeiten

- die praxisnahe Ausbildung der Lehramtsstudierenden

- Aufgeschlossenheit gegenüber Anwendungen der Mathematik. 
Schwächen sieht der Fachbereich in der Beschränkung der Breite des Lehrangebotes durch die Kleinheit des Fachbereichs. Wünschenswert wäre eine stärkere Verwendung neuer Medien in den Bereichen, wodurch das Verständnis für mathematische Inhalte gefördert wird. 


\subsection{Technische Universität Wien}

\section{Fakultät für Mathematik und Geoinformation}

\section{Teil A: Forschung, Wissenschaftlicher Nachwuchs}

\section{Institut für Analysis und Scientific Computing}

Das Institut für Analysis und Scientific Computing besteht aus den Forschungsgruppen:

- Numerik und Simulation von Differentialgleichungen

- Partielle Differentialgleichungen u. Dynamische Systeme

- Funktionalanalysis

- Mathematische Strukturanalyse und Interpolation

- Advanced Scientific Computing

- Mathematische Modellbildung und Simulation

- Computational Neuroscience and Biomedical Engineering

\section{Raum-, Sach-, Personalausstattung}

Das Institut verfügt über eine Raumnutzfläche von $1186 \mathrm{~m}^{2}$ für Personal und $613 \mathrm{~m}^{2}$ für sonstige Räume. In jedem Dienstzimmer und in den Praktikumsräumen stehen PCs, jedoch z.T. veraltet.

Das Institut verfügt über folgende Finanzmittel

\begin{tabular}{|l|r|r|r|}
\hline In $€$ & 2001 & 2002 & 2003 \\
\hline Laufende Ausgaben & $78.954,86$ & $81.049,50$ & $59.820,90$ \\
\hline Investitionen (Bundesmittel) & 30.196 .07 & $* 180.529,00$ & $* 76.451,00$ \\
\hline Einnahmen Bundesmittel & $109.150 .93,00$ & 261.578 .50 & 136.271 .90 \\
\hline Drittmittel über die Universität & $80.125,30$ & 191.076 .64 & $115.952,31$ \\
Drittmittel FWF & $216.415,90$ & $482.103,20$ & $609.415,90$ \\
Sonstige Drittmittel & $78.963,92$ & $178.873,24$ & $842.696,79$ \\
\hline
\end{tabular}

* Berufungszusagen (2002: 145.000 - 2003: 72.700)

Das Institut verfügt über 29,5 Personalstellen, die aus Haushaltsmitteln finanziert werden (5 Professuren, 19 wiss. Personal, 5,5 nichtwiss. Personal) und 18,5 Stellen, die drittmittelfinanziert sind (18 wiss. Personal, 0,5 Sekretariat).

Das Institut sieht Defizite bei der Rechnerbetreuung (Hardware/Software), da hierfür kein Personal zur Verfügung steht. Die Geräte seien größtenteils veraltet und es fehle das Budget 
für Reparaturen bzw. Neuanschaffungen. Zudem gäbe es zu wenig Platz für Projektmitarbeiter.

\section{Publikationen}

Der Publikationsumfang des gesamten Institut lag in den letzten 3 Jahren bei 298 Publikationen (Zeitschriftenaufsätze, Monographien, Proceedingsbeiträge). Es wurden 205 Vorträge gehalten.

\section{Wissenschaftlicher Nachwuchs}

In den letzten 3 Jahren wurden insgesamt ca. 70 Promotionen abgelegt, darunter ca. 1/3 von extern. Im gleichen Zeitraum gab es 2 Habilitationen, die im Zeitraum von 8 Jahren geschrieben wurden. Das Durchschnittsalter lag bei 35 Jahren. Doktoranden und Habilitanden werden, wenn möglich, durch Tutorien, Seminare, Besuche von Tagungen und Projektarbeit in die Lehre einbezogen. Es gibt das Graduiertenstudium Differential Equations im Wissenschaftskolleg.

\section{Personalfluktuation}

Es haben 6 Wissenschaftler das Institut verlassen. Davon hat 1 einen Ruf an eine andere Universität angenommen, die anderen 5 sind in Wirtschaftsunternehmen gewechselt. Das Institut sieht langfristig eine katastrophale Personalreduktion wegen der verzerrten Altersstruktur bei den Universitätsprofessoren auf sich zukommen.

\section{Kooperationen in der Forschung}

Das Institut unterhält zahlreiche Kooperationen mit in- und ausländischen Universitäten (z.B. Karolinska Institut Schweden, RUG Groningen, NL, University of Tennessee) und Forschungseinrichtungen (z.B. IBM T.J.Watson Research Center, Yorktown, USA, FZ Jülich, D). Darüber hinaus beteiligt es sich an verschiedenen nationalen und internationalen Forschungsprojekten.

\section{Anerkennung der Leistungen des Fachbereichs}

Die Mitglieder des Instituts waren in verschiedener Funktion als Gutachter im In- und Ausland tätig und haben sich als Mitherausgeber von Zeitschriften und als Mitglieder von Forschungsbeiräten engagiert. Eine Reihe von nationalen und internationalen Preisen und Auszeichnungen gingen an Mitglieder des Instituts. 


\section{Institut für Diskrete Mathematik und Geometrie}

Das Institut für Diskrete Mathematik und Geometrie besteht aus den 6 Forschungsgruppen:

- Algebra

- Computational Logic

- Differentialgeometrie und Geometrische Strukturen

- Geometrische Modellierung und Industrielle Geometrie

- Kombinatorik und Algorithmen

- Konvexe und Diskrete Geometrie

\section{Raum-, Sach-, Personalausstattung}

1 PC pro Mitarbeiter, File- und Internetserver, Netzwerke, 5 Netzwerkdrucker, Scanner, 40

PCs für Lehrveranstaltungen.

Das Institut verfügt über folgende Finanzmittel:

\begin{tabular}{|l|r|r|r|}
\hline in $€$ & 2001 & 2002 & 2003 \\
\hline Laufende Ausgaben & 94.500 & 94.600 & 79.200 \\
\hline Investitionen(Bund) & 32.800 & 20.100 & 1.800 \\
\hline Investitionen (Drittmittel) & 7.900 & 9.000 & 6.800 \\
\hline Einnahmen Bundesmittel & 127.300 & 114.700 & 81.000 \\
\hline Drittmittel über die Universität & 146.500 & 156.700 & 48.700 \\
\hline Drittmittel FWF & 153.000 & 265.000 & 482.000 \\
\hline
\end{tabular}

Das Institut verfügt über 41,16 Stellen, die aus Haushaltsmitteln bezahlt werden (6 Professuren, 1 Professor ist beurlaubt, 29 wiss. Personal, (davon 5 auf Zeit), 6,16 nichtwiss. Personal), weiters 9 zusätzl. Lehrbeauftragte und 11 externe Dozenten, deren Lehraufgaben von Haushaltsmitteln bezahlt werden. 18,5 wissenschaftliche Projekt-Stellen sind drittmittelfinanziert. Die personelle Ausstattung ist nach Meinung des Institutes derzeit noch ausreichend. Durch die Sparmaßnahmen der letzten 10 Jahre seien jedoch zahlreiche Stellen nicht nachbesetzt worden. In den nächsten Jahren müsste noch mehr gespart werden. Damit seien personelle Engpässe vorprogrammiert. Es stünden daher seit einem Jahrzehnt kaum Stellen aus Haushaltsmitteln für junge Absolventen zur Verfügung. Es bestünden Abweichungen zwischen Stellen-Soll und -Ist von 4,5 Stellen wg. Einsparungen. 
Es gibt 41 Räume für Personal, 8 Seminarräume und 3 Computerräume auf insgesamt $1060 \mathrm{~m}^{2}$ Nutzfläche. Nach Ansicht des Institutes ist die Bibliothek gut ausgestattet mit OnlineZeitschriften, Computern, Büchern und Zeitschriften auch über Fernleihe. Durch die Budgetkürzungen mussten Zeitschriften jedoch abbestellt werden.

Nach Ansicht des Institutes ist die räumliche und gerätemäßige Ausstattung ausreichend. Es wird jedoch eine qualitative Verbesserung gewünscht. Das Institut bemängelt, dass es keine institutionalisierten Gästemittel gibt.

\section{Besondere Aktivitäten in der Lehre}

Der wissenschaftliche Nachwuchs wird in Seminare und laufende Projekte eingebunden.

Darüber hinaus werden Lehrerfortbildungen veranstaltet und die Österreichische Mathematische Olympiade wissenschaftlich betreut.

\section{Publikationen}

Die Publikationen der Institutsmitglieder (in den Jahren 2001-2003) umfassen 273Arbeiten (Monographien und Aufsätze in Zeitschriften und Tagungsbänden). Es wurden insgesamt 558 Vorträge gehalten.

\section{Wissenschaftlicher Nachwuchs}

Im Zeitraum 1999 bis 2003 wurden 17 Promotionen abgelegt, 1 davon extern. Die durchschnittliche Promotionsdauer lag zwischen 3 und 4 Jahren. Das Durchschnittsalter betrug ca. 28 Jahre. Im gleichen Zeitraum gab es 7 Habilitationen mit einer Erarbeitungszeit von ca. 6-7 Jahren. Das Durchschnittsalter lag hier bei 33 Jahren. Alle wissenschaftlichen Mitarbeiter, auch Doktoranden und Habilitanden werden mit entsprechender Lehre betraut. Am mathematischen Fachbereich existiert ein Doktoratsstudium.

\section{Personalfluktuation}

In den letzten 5 Jahren haben 11 Wissenschaftler die Universität verlassen ( 4 haben Rufe an andere Universitäten angenommen) und weitere 10 Projektassistenten. Im genannten Zeitraum gab es keine Berufungen und nur 6 Neuzugänge von Universitätsassistenten (teilweise nur auf Zeit).

Die Karrieremöglichkeiten an der TU Wien sind sehr beschränkt. Es konnten nur wenige Stellen ausgeschrieben werden, diese wurden mit qualifizierten Mitarbeitern besetzt. 


\section{Kooperation in der Forschung}

Die einzelnen Forschungsgruppen des Instituts unterhalten vielfältige Kooperationen mit anderen Universitäten sowie zahlreichen nicht-universitären Institutionen im In- und Ausland. Anerkennung der Leistungen des Fachbereichs

Die Mitglieder des Instituts waren in verschiedener Funktion als Gutachter im In- und Ausland tätig und haben sich als Mitherausgeber von Zeitschriften und als Mitglieder in Beiräten und Vorständen engagiert. Eine Reihe von nationalen und internationalen Preisen und Auszeichnungen gingen an Mitglieder des Instituts.

\section{Institut für Wirtschaftsmathematik}

Dem Institut gehören folgende Forschungsgruppen an:

- 1. Finanz- und Versicherungsmathematik

- 2. Ökonometrie und Systemtheorie

- 3. Operations Research und nichtlineare dynamische Systeme

- 4. Ökonomie

An der Evaluation beteiligt sich nur die Forschungsgruppe Finanz- und Versicherungsma-

\section{thematik.}

Raum-, Sach-, Personalausstattung

Für die Lehre stehen zur Verfügung: 1 PC pro Mitarbeiter, 3 PCs für Gäste und Diplomanden, 7 Laptops, File- und Internetserver, Netzwerke, 4 Netzwerkdrucker, Scanner, Beamer etc.)

Die Forschungsgruppe verfügt über folgende Finanzmittel:

\begin{tabular}{|l|r|r|r|}
\hline in $€$ & 2001 & 2002 & 2003 \\
\hline laufende Ausgaben & $32.616,-$ & $26.300 .-$ & $16.610,-$ \\
\hline Investitionen (Bundesmittel) & $36.772,-$ & $37.800 .-$ & $14.990,-$ \\
\hline Investitionen (Drittmittel) & k.A. & $10.000,-$ & k.A. \\
\hline Einnahmen Bundesmittel & $69.388,-$ & $64.100,-$ & $31.600,-$ \\
Drittmittel über die Universität & $3.625,-$ & $978,-$ & $3.758,-$ \\
Drittmittel FWF & $261.678,-$ & $246.380,-$ & $238.312,-$ \\
\hline Sonstige Drittmittel & & & \\
Jubiläumsfond, ÖNB & $25.437,-$ & $47.060,-$ & $46.308,-$ \\
EU-Projekt via WPI & & & $54.927,-$ \\
\hline
\end{tabular}


Die Forschungsgruppe besteht aus 16 Personen, die aus Haushaltsmitteln finanziert werden (2 Professoren, , 4 wiss. Personal (davon 1 auf Zeit) , 8 externe Lehrbeauftragte, 2 nichtwiss. Personal (mit 1,5 Stellen)) und 9,5 Personalstellen, finanziert aus Drittmitteln.

Die Forschungsgruppe verfügt über 8 Räume für wiss. Mitarbeiter, 2 Räume für adm. Personal, 1 kleinen Seminarraum und 1 Serverraum verteilt auf 324,93 $\mathrm{m}^{2}$ Nutzfläche. Es gibt keine Abweichungen zwischen Stellen-Soll und -Ist.

Als größte Stärke sieht die Forschungsgruppe ihre personelle Situation, die mit hoher Motivation die Aufgaben in Forschung und Lehre wahrnimmt. Auch die sächliche Ausstattung (insbesondere IT) wird als durchaus zufriedenstellend zu bezeichnet. Der größte Engpass sei zur Zeit in der räumlichen Ausstattung gegeben, die in keiner Weise den in den vergangenen Jahren stark gewachsenen Aufgaben der Gruppe entspräche.

Das neue Institut für Wirtschaftsmathematik wurde erst 2004 gegründet. Es entstand aus mehreren Vorgängerinstituten der TU Wien und ist räumlich zerrissen und auf 3 Gebäude verteilt. Eine Zusammenlegung am Standort Argentinierstrasse sollte nach Meinung der Gruppe angestrebt werden.

\section{Besondere Aktivitäten in der Lehre}

Es gibt ein engmaschiges Netz an Zusammenarbeit innerhalb der Forschungsgruppe im Rahmen der Aus- und Weiterbildung, die in Projekten, Seminaren, Privatissima etc. ihren organisatorischen Rahmen finden.

\section{Praxisbezug}

Das Institut steht in engem Kontakt mit Banken und Versicherungen (externe Lektoren, Projekte, Praktika etc.).

Auf der Institutsseite werden unter $h t t p: / / w w w . f a m . t u w i e n . a c . a t / j o b s / i n d e x . p h p \_p r o$ Jahr etwa 40 Stellen aus der Finanzindustrie angeboten.

\section{Publikationen}

Die Forschungsgruppe hat im Zeitraum 2001-2003 folgenden Publikationsumfang erbracht: 68 Beiträge, (davon 41 Aufsätze in referierten Zeitschriften, des weiteren 14 Beiträge in Monografien und Tagungsbänden). Im gleichen Zeitraum wurden 238 Vorträge gehalten, davon 140 auf Einladung.

\section{Wissenschaftlicher Nachwuchs}

Im Zeitraum 1999 - 2003 gab es 4 Promotionen und 2 Habilitationen. Die Nachwuchswissenschaftler werden in die Lehre eingebunden, wobei aber für junge Mitarbeiter/innen der 
Schwerpunkt auf den Forschungsaufgaben liegt. Zur Vorbereitung auf die Lehraufgaben dient vor allem die intensive Auseinandersetzung im Rahmen von Seminaren und Privatissima.

\section{Personalfluktuation}

In den letzten 5 Jahren sind 23 Abgänge zu verzeichnen, 17 davon haben andere Stellenangebote bekommen, 6 haben Rufe an andere Universitäten erhalten. Im gleichen Zeitraum sind 4 Berufungen zu verzeichnen.

Aufgrund von Rufabwehrverhandlungen ist es gelungen, dass 2002 eine Professorenstelle ausgeschrieben werden konnte. Bei der Berufung selbst gab es wenig Schwierigkeiten und deren Ausgang ist als sehr geglückt zu bezeichnen.

Aufgrund der starken internationalen Vernetzung der Gruppe war es bisher immer möglich, sehr qualifizierte Mitarbeiter/innen (z.Z. 4 aus Haushalts- und 7 aus Drittmitteln finanzierte wissenschaftliche Mitarbeiter/innen) zu finden. Im Rahmen dieses jungen Teams gab es bisher auch keine Schwierigkeiten, diese zu halten, abgesehen von der normalen Fluktuation bei Projektmitarbeitern/innen (z.B. Post Docs) oder aufgrund einer akademischen Karriere .

Insgesamt sieht die Forschungsgruppe die Ausstattung mit 2 Professorenstellen und einem jungen, hochmotivierten Team im Rahmen der schwierigen Bedingungen an der TU Wien für die Durchführung der bisherigen Lehr- und Forschungstätigkeiten - als durchaus zufriedenstellend. Für neue Aufgaben, die zur Stärkung des innovativen Schwerpunkts Finanz- und Versicherungsmathematik wesentlich scheinen, wäre allerdings auch eine entsprechende Erweiterung der personellen Ausstattung notwendig.

\section{Kooperation in der Forschung}

Die Forschungsgruppe unterhält vielfältige Kooperationsbeziehungen mit anderen Hochschulen und staatlichen Institutionen in Österreich (z.B. Österreichische Nationalbank, Universität Wien) sowie verschiedenen Hochschulen im EU-Raum und in den USA (z.B. ETH Zürich, Schweiz, Humboldt Universität Berlin, D; London Imperial College, UK; Stanford University, USA).

\section{Anerkennung der Leistungen des Fachbereichs}

Die Mitglieder der Forschungsgruppe waren in verschiedener Funktion als Gutachter für nationale und internationale Forschungsförderfonds tätig und haben sich als Mitherausgeber von Zeitschriften und als Mitglieder in Beiräten und Vorständen engagiert. Eine Reihe von nationalen und internationalen Preisen und Auszeichnungen gingen an Mitglieder des Instituts. 


\section{Institut für Statistik und Wahrscheinlichkeitstheorie}

Das Institut besteht aus den Forschungsgruppen

- Computational Statistics

- Stochastische Methoden

\section{Raum-, Sach-, Personalausstattung}

Dem Institut stehen 17 Räume für wissenschaftliches Personal und Gäste auf einer Nutzfläche von $725 \mathrm{~m}^{2}$ zur Verfügung. Für die Lehre stehen zur Verfügung 20 PCs, 7 Notebooks, 5 Laserdrucker, 1 Kopierer.

Das Institut verfügt über folgende Finanzmittel:

\begin{tabular}{|l|r|r|r|}
\hline in $€$ & 2001 & 2002 & 2003 \\
\hline laufende Ausgaben (o.Dot.) & $34.426 .-*$ & $22.950 .-$ & $19.650 .-$ \\
\hline Investitionen (Bundesmittel) (o.Dot) & $20.261 . .^{*}$ & $10.550 .-$ & $1.500 .-$ \\
\hline
\end{tabular}

*gemeinsam mit der Abteilung Finanz- und Versicherungsmathematik

Sonstige Drittmittel (über Projekte):

- Zeitreihenanalyse mit unscharfen Daten, 2 Jahre, ÖNB-Jubiläumsfonds, $41.041 €$

- Computerunterstützte Statistische Messwertanalyse, 2 Jahre, FWF, $28.335 €$

- e-Direct Marketing Solution, 1 Jahr, FFF und Upper Network GmbH, $20.000 €$

- Erhöhte Sicherheit in der funktionellen Diagnostik, 1,5 Jahre, ÖNB, $50.871 €$

- Euro Conference on Robust Statistics, EU, $34.785 €$

- Adaptive Modelle in den Sozial- und Wirtschaftswissenschaften, 7 Jahre, FWF, $369.000 €$

Das Institut hat 18 Stellen, die aus Haushaltsmitteln finanziert sind (3 Professoren, 8 wiss.

Personal, 4 zusätzliche Lehrbeauftragte, 3 nichtwiss. Personal) und 4 wiss. Stellen, die aus

Drittmitteln finanziert werden. Es gibt eine Vakanz wegen fehlender Besetzbarkeit.

Am Institut existieren 2 Bibliotheken, 2 Seminarräume, 1 Küche, 1 Sozialraum. Es wird ein

Gemeinschaftsraum im Bereich Wiedner Hauptstraße 8-10 als fehlend bemängelt.

\section{Publikationen}

Das Institut hat folgenden Publikationsumfang im Zeitraum 2001-2003 geleistet: 127 Publikationen. Im gleichen Zeitraum wurden 85 Vorträge gehalten. 
Wissenschaftlicher Nachwuchs

In den letzten 5 Jahren wurden 13 Promotionen abgelegt mit einer durchschnittlichen Dauer von 3 Jahren und einem Durchschnittsalter von 28 Jahren. Im gleichen Zeitraum gab es 1 Habilitation, die in 5 Jahren geschrieben wurde. Der Habilitand war 33 Jahre alt. Doktoranden und Habilitanden werden als Tutoren in Übungen eingesetzt. Als Graduiertenstudium existiert das Doktoratsstudium.

\section{Personalfluktuation}

In den letzten 5 Jahren haben 2 Wissenschaftler das Institut verlassen. 1 Vertragsassistent wurde eingestellt.

Kooperationen in der Forschung

Das Institut unterhält langfristige Kooperationen mit dem Institut für Mathematische Stochastik der TU Dresden.

Anerkennung der Leistungen des Fachbereichs

Vom einem Institutsmitglied wurde Gutachtertätigkeiten für den FWF und die DFG ausgeübt. Des weiteren bestehen zahlreiche Tätigkeiten bei der Mitherausgabe von Zeitschriften. Ein Institutsmitglied wurde von der WU Wien mit dem Best Paper Award ausgezeichnet. 


\section{Teil B: Lehre und Studium an der Fakultät für Mathematik und Geoinformation der TU Wien}

Ausbildungsprofil

Die Fakultät für Mathematik und Geoinformation bietet mit dem Studiengang TMA ein 5jähriges Diplomstudium mit Abschluss „Dipl.-Ing.“ (160 SWS) an. Ab dem 3. Semester findet die Aufspaltung in 5 Studienzweige statt mit teilweise gemeinsamen Lehrveranstaltungen (Betreuung durch Angehörige der Mathematikinstitute):

- Mathematik in den Naturwissenschaften

- Wirtschaftsmathematik

- Mathematik in den Computerwissenschaften

- Finanz- und Versicherungsmathematik

- Statistik (neu)

Die Versicherungsmathematik bietet ein 3-jähriges Bakkalaureatstudium mit anschließendem 2-jährigen Magisterstudium an.

Darüber hinaus gibt es die Lehramtsstudiengänge Mathematik und Darstellende Geometrie.

\section{Allgemeine Ziele}

Das Diplomstudium Technische Mathematik bereitet durch eine wissenschaftlich fundierte Ausbildung auf eine Tätigkeit in Technik, Wirtschaft, Verwaltung und Forschung vor. Die AbsolventInnen erwerben auf der Basis fundierter Kenntnisse die Fähigkeit, mathematische und formale Strukturen einer Problemstellung zu erfassen und Lösungen zu erarbeiten. Schwerpunkte der Ausbildung sind zweigspezifische algorithmische Lösungsverfahren, Lösungsmethoden unter Einsatz von mathematischer Software sowie Aufbereitung und Bewertung der Ergebnisse für die Erfordernisse im Berufs- und Wirtschaftsleben.

Die Studienziele und -inhalte sind im Studienplan dokumentiert. Die Studienkommission tagt mehrmals im Semester, um eventuell notwendige Korrekturen oder Modifikationen im Studienplan wahrzunehmen.

Die Schwerpunktbildung wird durch die 5 Studienzweige ermöglicht. Eine feinere Schwerpunktbildung entsteht durch die gebundenen Wahlfächer, von denen 30 Stunden aus 9 Katalogen zu wählen sind, davon aber mindestens 15 aus einem einzigen Katalog. 


\section{Praxisbezug}

Das Studium der Technischen Mathematik wird als Berufsvorbildung verstanden und nicht als Berufsausbildung. Daher steht der Praxisbezug im Studium selbst nicht im Vordergrund. Es gibt aber LVA's von Praktikern im Rahmen der Wahl- und Freifächer, etwa in der Informatik.

Ein erhöhtes Interesse von Studenten an mehr praxisbezogenen bzw. mehr forschungsbezogenen Lehrveranstaltungen ist schwer zu ermitteln wegen des großen Spektrums an angebotenen Wahlfächern nach Aussage der Fakultät.

Die Studienkommission unterstützt die Mobilität der Studierenden in Europa. Die Fakultät bietet Ihren AbsolventInnen die Möglichkeit eines Doppeldiploms mit der Ecole Centrale in Paris.

Es werden jedoch Zweifel an der Sinnhaftigkeit eines Bakkalaureatstudiums geäußert, gerade in der Mathematik. Hier sieht die Studienkommission das 5-jährige Diplomstudium als sinnvoller an. Ausnahme: das Bakkalaureatstudium Versicherungsmathematik, welches für das spezifische Berufsbild sinnvoll erscheint.

\section{Personelle Ressourcen}

Es liegen nur Angaben zu den personellen Ressourcen der Institute Diskrete Mathematik und Geometrie und der Forschungsgruppe Finanz- und Versicherungsmathematik vor.

Beim Institut für Diskrete Mathematik umfasst die Gesamtlehrkapazität des festen Lehrkörpers (7 Professoren, 22 Dozenten, 8 Assistenten, 16 Berufspraktikern und einigen Externen) 650 SWS.

Bei der Forschungsgruppe Finanz- und Versicherungsmathematik ist eine Gesamtlehrkapazität von 104 SWS vorgesehen, die von 2 Professoren, 3 Assistenten und LektorInnen und 8 Berufspraktikern durchgeführt werden. Das tatsächliche Lehrangebot umfasste im Studienjahr 2003/2004: 124 SWS.

An Serviceleistungen, die die Mathematikinstitute für Studiengänge anderer Studien gehalten haben, wurden im letzten Studienjahr 1040 Stunden errechnet. Demgegenüber stehen 21 Stunden, die von Nichtmathematikern für Mathematikstudenten gegeben wurden.

Gewisse Lehrveranstaltungen anderer Institute können als Wahlfächer gewählt werden und beliebige Lehrveranstaltungen als Freifächer. 


\section{Sachausstattung}

Es liegen die Zahlen für das Institute Diskrete Mathematik und Geometrie und der Forschungsgruppe Finanz- und Versicherungsmathematik vor. Demnach gibt es insgesamt 43 PC-Arbeitsplätze in 3 Computerräumen. Laborarbeitsplätze sind nicht vorhanden.

Die Bibliothek der Forschungsgruppe Finanz- und Versicherungsmathematik ist durch Verleihung des Wittgensteinpreises sowie durch Berufungsmittel mit grundlegender Literatur wie auch an spezialisierter Forschungsliteratur sehr gut bedient. Die Aktualisierung erfolgt regelmäßig und umfassend, darüber hinaus wird sie mit den Lehrenden in Bezug auf Lehrveranstaltungsthemen und Prüfungsstoffe abgestimmt. Der solcherart ständig erweiterte Bestand wird von Studierenden wie auch von Praktiker/innen intensiv genützt.

Studienanfänger $(w / m)$

\begin{tabular}{|r|r|r|r|r|r|}
\hline Studiengang & 1999 & 2000 & 2001 & 2002 & 2003 \\
\hline Technische Mathematik & & & & & \\
1.FS & $50 / 142$ & $82 / 178$ & $73 / 163$ & $58 / 157$ & 203 \\
Nach 1 Jahr & $32 / 105$ & $56 / 134$ & $33 / 122$ & & \\
\hline
\end{tabular}

Studierende (w/m) (Berechnungsgrundlage 2002)

\begin{tabular}{|l|r|r|r|r|r|r|r|r|r|}
\hline Studiengang & \multicolumn{6}{|l|}{ Studienrende im .............. Fachsemester } \\
\hline Technische Mathematik & 1. & 2. & 3. & 4. & 5. & 6. & 7. & 8. & 9. \\
\hline & $59 / 157$ & $43 / 127$ & $47 / 115$ & $21 / 81$ & $16 / 53$ & $8 / 22$ & $7 / 31$ & $9 / 14$ & $6 / 13$ \\
\hline
\end{tabular}

\section{Studien- und Prüfungsorganisation}

$\mathrm{Zu}$ allen Vorlesungen des ersten Studienabschnitts sowie zu fast allen Vorlesungen des zweiten Abschnitts werden Übungen angeboten, die meist mit Gruppen von 15-30 Studierenden abgehalten werden. Die Übungen sind ebenfalls Pflichtlehrveranstaltungen. Zu allen Pflichtvorlesungen der ersten 3 Semester werden Repetitorien angeboten, dies ist durch den Studiengang vorgeschrieben. Die Gesamtstundenzahl der zu absolvierenden Prüfungen beträgt 160 SWS, davon sind 114 Pflichtfächer, 30 gebundene Wahlfächer und 16 freie Wahlfächer (Freifächer). Im ersten Studienabschnitt gibt es nur Pflichtfächer.

Es gibt keine Fächer, die formal als Kernfächer ausgezeichnet sind. Allerdings gibt es Pflichtlehrveranstaltungen, die allen 5 Studienzweigen gemeinsam sind, und andere Pflichtlehrver- 
anstaltungen, die nur in einem Zweig Pflicht sind (letztere sind dann in allen anderen Zweigen automatisch als gebundene Wahlfächer wählbar.)

Die Lehrveranstaltungen können in beliebiger Reihenfolge absolviert werden. Im Studienplan empfohlen ist für jedes Pflichtfach 1 Semester. Dieselbe Vorlesung wird so gut wie nie parallel angekündigt.

Große Vorlesungen werden im allgemeinen nicht von derselben Person gehalten, die sie letztes Jahr gehalten hat, man wechselt sich im 2- oder 3-Jahresrhythmus ab. Alle Pflichtvorlesungen und die meisten Übungen werden von Habilitierten gehalten, das sind sowohl Universitätsprofessoren als auch a.o. Universitätsprofessoren.

Alle Lehrgebiete werden regelmäßig angeboten. Der Studiendekan ist verpflichtet, dafür zu sorgen, dass die für das Studium notwendigen LVA auch angekündigt werden.

\section{Prüfungsverfahren}

Für jede LVA müssen pro Semester mindestens 3 Prüfungs-Termine angeboten werden. Dieser werden über die Webpage des Institutes oder des Prüfers bekannt gegeben sowie in einem Schaukasten des Institutes ausgehängt. Mündliche Prüfungen werden oft informell und kurzfristig vereinbart. Prüfer ist der Vortragende der Lehrveranstaltungen.

In der Technischen Mathematik ist es nicht üblich, eine „Abschlussarbeit“ für einzelne Lehrveranstaltungen zu schreiben. Die Aufgabenstellung für die Diplomarbeit ist so zu wählen, dass der Studierende die Bearbeitung innerhalb von 6 Monaten leisten kann. Die Anmeldung zu schriftlichen Prüfungen ist im Allgemeinen bis zu wenigen Tagen vor dem Prüfungstermin möglich.

Wartezeiten gibt es im Studiengang Technische Mathematik nicht. Die mindestens 3 Prüfungstermine sind über das Semester verteilt, der Studierende muss höchstens 2 Monate auf einen neuen Termin warten.

Die Frist zwischen schriftlicher und mündlicher Prüfung liegt bei wenigen Tagen nach dem schriftlichen Teil. Bei mündlicher Prüfung wird die Note direkt bekannt gegeben. Bei schriftlicher Prüfung nach ca. 1-4 Wochen nach der Prüfung.

Im ersten Studienabschnitt dürfen Prüfungen 3 Mal wiederholt werden, später dann 4 Mal.

\section{Leistungsanforderungen}

Die Gesamtstundenzahl der zu absolvierenden Prüfungen beträgt in jedem Studienzweig 160 SWS.

Davon entfallen auf den 1. Abschnitt (2 Semester) 33 SWS.

Auf den 2. Studienabschnitt (4 Semester) je nach Studienzweig 68-72 Pflichtstunden. 
Auf den 3.Studienabschnitt (4 Semester) 9-13 Pflichtstunden

Hinzu kommen 30 SWS für die gebundenen Wahlfächer

16 SWS für freie Wahlfächer, sowie die Diplomarbeit.

In den Übungen müssen ständig Leistungsnachweise erbracht werden. In den meisten Übungen werden pro Woche Übungsaufgaben gestellt, die bis zur Woche darauf gelöst sein müssen. Der Leistungsnachweis in einem Praktikum besteht im Lösen eines praktischen Problems (meist durch Programmierung).

Das Studium ist innerhalb der vorgesehenen Regelstudienzeit (10 Semestern) durchführbar. Es gibt bisweilen Studierende, die das Studium in nur 8 oder 9 Semestern abschließen.

\section{Studienzeiten}

Die durchschnittlichen Studienzeiten bis zum Abschluss des Studiums lag im Zeitraum 19931997 zwischen 6 und 7 Jahren. Die Anzahl der Studierenden, die innerhalb der Regelstudienzeit das Studium abschließen, ist sehr gering.

Die Berufschancen von AbsolventInnen werden als ausgezeichnet eingeschätzt und werden von der Industrie wegen ihrer Problemlösungsfähigkeit den Informatikabsolventen vorgezogen.

\section{Qualitätssicherung}

Über die Situation in der Lehre wird in der Studienkommission diskutiert, die je nach Bedarf mehrmals im Semester tagt. An den Sitzungen der Studienkommission nimmt der Studiendekan regelmäßig teil. Die Mindestinhalte der Pflichtlehrveranstaltungen sind im Studienplan festgehalten.

Die Studierenden können Kommentare und Bewertungen von LVA im Rahmen einer WWWbasierten anonymisierten Evaluation abgeben; die Ergebnisse werden dem LVA-Leiter und dem Studiendekan bekannt gegeben.

Es gibt Grundkurse für StudentInnen, in denen Grundkenntnisse aus der reellen Analysis vermittelt werden. Die Vorlesungen werden in den ersten Semestern von Repetitorien und Konversatorien begleitet.

Bei Habilitanden werden 2 Gutachten über die didaktische Qualifikation des Habilitanden verfasst, mindestens eine davon von Studierenden. Ohne eine positive Bewertung der didaktischen Fähigkeiten ist eine Habilitation unmöglich. Bei Berufungen spielt vor allem die wissenschaftliche Qualifikation eine Rolle. Kandidaten, die in die engere Wahl kommen, müssen einen öffentlichen Vortrag halten; die bei diesem Vortrag erkannten didaktischen Fähigkeiten machen im Allgemeinen auch einen großen Eindruck auf die Berufungskommission. 


\section{Gesamteinschätzung der Fakultät}

Die Fakultät sieht die Stärken und Schwächen der Ausbildung wie folgt:

Stärken:

- Breites Angebot an Wahlfächern

- Rotation der Lehrveranstaltungs-Leiter der Grundvorlesungen

- Spezialvorlesungen meistens vor Experten in ihrem Gebiet, durch besondere Nähe zur Front der Forschung.

Schwächen sieht die Fakultät vor allem personenbezogen. Die Qualität der Ausbildung wird von der Fakultät als gut bezeichnet. 


\subsection{Universität Wien}

\section{Teil A: Forschung, Wissenschaftlicher Nachwuchs}

\section{Institut für Mathematik}

\section{Raum-, Sach-, Personalausstattung}

Das Institut verfügt über 238 PCs , 50 High End PCs/Server/Cluster, 35 Notebooks (teilweise sehr veraltet), 15 Drucker sowie 5 Beamer und diverse Kleingeräte.

Die Fakultät verfügt über folgende Finanzmittel

\begin{tabular}{|l|r|r|r|}
\hline in $€$ & 2001 & 2002 & 2003 \\
\hline Laufende Ausgaben & $55.237,00$ & $64.590,00$ & $52.880,00$ \\
\hline Investitionen (Bundesmittel) & $60.573,00$ & $80.000,00$ & $15.000,00$ \\
\hline Drittmittel über die Universität & $120.902,96$ & $458.662,50$ & $1.381 .006,00$ \\
Drittmittel FWF & $804.198,64$ & $730.181,51$ & $1.195 .660,16$ \\
& & & \\
\hline Sonstige Drittmittel & $124.100,34$ & $52,580,00$ & $354.728,16$ \\
\hline
\end{tabular}

Nach Meinung des Instituts ist die personelle Ausstattung in Anbetracht der umfangreichen Anforderungen in Forschung und Lehre sehr schlecht. Die Fakultät verfügt über 62 aus Haushaltsmitteln finanzierte Stellen (59 Vollzeit + 3 Teilzeit) (11 Professoren, 29,5 wiss. Personal, 2,5 wiss. Mitarbeiter auf Zeit, (6) 5 zusätzliche Lehrbeauftragte (darunter drei Honorarprofessoren), 9,5 Sekretariat und Verwaltung und 2 Technische Dienste). Darüber hinaus gibt es 45 ForschungsassistentInnen und 2 Stellen im Technischen Dienst, die über Drittmittel finanziert werden.

Die räumliche Ausstattung wird vom Institut als ungenügend betrachtet: Seit SS 2004 belegt das Institut für Mathematik Räume in den Stockwerken 4 bis 7 im Turm C des Gebäudes Nordbergstraße 15 (vorher Verteilung der Abteilungen auf 7 verschiedene Standorte). Dem Institut gehören: 69 Dienstzimmer (3 davon im 1. Stock), 4 Besprechungs- bzw. Prüfungszimmer, 5 Sekretariatszimmer, 3 PC-Labors, 5 Archive, 4 Teeküchen und 1 Küche. Zusätzlich im 2. Stock ein StudentInnensekretariat und 3 PC-Labors für die Lehre. Die Gesamtfläche beträgt ca. $2.572 \mathrm{~m}^{2}$. Im 7. Stock ist außerdem das Wolfgang-Pauli-Institut (WPI) mit 9 Dienstzimmern untergebracht. Diese Kapazität reicht aber bei weitem nicht aus, da neue Forschungsprojekte mit vielen ForschungsassistentInnen hinzugekommen sind. 
Der Bestand der Bibliothek wird im großen und ganzen als gut angesehen, einerseits da die Bibliothek eine der ältesten Fachbibliotheken in Österreich ist und jahrzehntelang die klassischen Zeitschriften sammeln konnte, anderseits und vor allem aber wegen jährlicher Subventionen durch Institutsmitglieder in Höhe von durchschnittlich 65.000 Euro als Ergebnis einer umfangreichen Rezensionstätigkeit, der Herausgabe mehrerer Zeitschriften und des Engagements Nachlässe betreffend.

Aufgrund von Sparmaßnahmen ist jedoch eine Aktualisierung des Bestandes mit neuen Zeitschriften nicht mehr möglich. Das Institut wünscht sich einen Online-Zugang zu möglichst vielen der bisher nicht zugänglichen Zeitschriften.

\section{Besondere Aktivitäten in der Lehre}

Begabte StudentInnen werden schon früh in die einzelnen Arbeitsgruppen einbezogen und schrittweise individuell und im Team ihre wissenschaftliche Entwicklung bis zur Habilitation gefördert (incl. mit entsprechender Auslandsaufenthalte; viele starten dann Auslandskarrieren). DoktorandInnen und Habilitanden, die am Institut angestellt sind, halten Lehrveranstaltungen jeglicher Art und sind unterstützend bei Seminaren tätig. Die Didaktikgruppe bietet Kurse an, die die entsprechenden Fertigkeiten in der Lehre vermitteln. Die Fakultät bietet ein vom FWF genehmigtes und finanziertes Wissenschaftskolleg an (eines von insgesamt drei in Österreich im gesamten Universitätsbereich), in Kooperation mit der TU Wien. Dieses hat den Schwerpunkt "Differentialgleichungen" und stellt ein gezieltes Programm zur Förderungen von Doktoranden und Post Docs dar, in engerer Kooperation mit dem Erwin-

Schrödinger-Institut und dem Wolfgang-Pauli-Institut.

\section{Nachfrage nach den Absolventen auf dem Arbeitsmarkt}

Die Nachfrage ist sehr gut. Mathematiker arbeiten in vielen verschiedenen hoch-qualifizierten Bereichen (wie z.B. in der Unternehmensberatung), wobei weniger spezielle mathematische Kenntnisse als viel mehr die allgemeine Lernfähigkeit und Problemlösungskapazität geschätzt werden. Dabei genießt das (absolvierte) Mathematikstudium hohes Ansehen. Wie viele Rückmeldungen zeigen, gibt es daher trotz des angespannten Arbeitsmarktes fast keine arbeitslosen MathematikerInnen. Auch im Lehramt kommen die AbsolventInnen mit Mathematik zumindest in Teilzeitbeschäftigung unter.

\section{Publikationen}

Das Institut hat in den letzten 5 Jahren insgesamt 453 wissenschaftliche Publikationen veröffentlicht, davon 337 Artikel in internationalen Zeitschriften. Es wurden auf Einladung 572 Vorträge gehalten sowie 128 Vorträge in Mathematischen Kolloquien. 


\section{Wissenschaftlicher Nachwuchs}

In den letzten 5 Jahren wurden 32 Promotionen abgelegt mit einer durchschnittlichen Dauer von 3,2 Jahren. Das Durchschnittsalter lag bei 30,9 Jahren. Dem gegenüber stehen 15 Habilitationen mit einer durchschnittlichen Dauer von etwa 4 Jahren. Das Durchschnittsalter bei Habilitationsabschluss lag hier bei 34,15 Jahren.

\section{Personalfluktuation}

In den letzten 5 Jahren haben 23 Wissenschaftler die Universität Wien verlassen, wovon 11 an eine ausländische Universität berufen wurden. Demgegenüber stehen 11 Neuzugänge, davon 2 Berufungen.

Das neue Dienstrecht erschwert die Berufung von hervorragenden Wissenschaftern aus dem Ausland, primär wegen einer deutlichen Verschlechterung bei den Pensionen. Ein weiteres aktuelles Problem (derzeit laufen drei Berufungsverfahren) sind die nunmehrigen Professuren auf Zeit. Berufungen werden erschwert bzw. scheitern, wenn der zu Berufende z.B. einen Ruf in Deutschland erhält (selbst C3-Professuren in Deutschland werden wegen der unbefristeten Anstellung einer befristeten, verlängerbaren, sonst aber C4-wertigen Professur in Österreich vorgezogen).

Dies führte vor allem in diesem Jahr zu Absagen und erheblichen Verzögerungen bei der Besetzung einer Professorenstelle. Das Institut vermutet, dass sich die Situation noch verschärfen wird, wie sich bei zwei Ausschreibungen von Professorenstellen gezeigt hat. In letzter Zeit folgten mehrere a. Professoren einer Wegberufungen. Es gibt seitens der Universität diesbezüglich keine Bleibeverhandlungen. Die neuen befristeten, nicht verlängerbaren Mittelbaustellen tragen zur verstärkten Abwanderung ohne Chance auf Rückholung bei.

\section{Kooperation in der Forschung}

Das Institut unterhält zahlreiche Kooperationen zu Universitäten im In- und Ausland, über zahlreiche personelle Kontakte, den FWF, NSF, im Rahmen von EU-Netzwerken, wo Institutsangehörige die Koordinatoren sind, z.B. zwei IHP Research-Netzwerke „ACE“ , HYKE, ein IST Netzwerk "COCONUT“, ein ALFA-Netzwerk, ein INTAS-Netzwerk, ein ESF-

\section{Netzwerk, HASSIP und NetAGES.}

Der Kooperation dienen auch Industrie-Stipendien (LEONARDO), IAESTE-StudentInnen, ERASMUS-Programm zum Studentenaustausch und Beziehungen zu anderen Forschungseinrichtungen. 
Ein Abkommen mit dem Rektorat über die Aufnahme chinesischer MathematikstudentInnen ist vor dem Abschluss. Weiter ist eine Kooperation mit den Universitäten Prag, Budapest und Krakau startbereit (CEEPUS).

Das Institut stellt den Präsidenten und einen der beiden Direktoren des Erwin-SchrödingerInstituts (ESI), den Präsidenten und Direktor des Wolfgang-Pauli-Instituts (WPI), den Direktor des "Institut CNRS Pauli" (UMI 2842 des CNRS) den stellvertretenden Direktor des Radon Instituts, ein Mitglied des wissenschaftlichen Beirats der Forschungsinstituts Oberwolfach, den für Naturwissenschaften und Technik zuständigen Vizepräsidenten des Forschungsförderungsfond (FWF).

Anerkennung der Leistungen des Fachbereichs

Viele Mitglieder des Instituts waren in verschiedener Funktion als GutachterInnen und Herausgeber von Zeitschriften im In- und Ausland tätig.

Eine Reihe wichtiger nationaler und internationaler Ehrungen ging an einige Mitglieder des Instituts.

Darunter sind die Marie Curie Excellence-Professur, 1,8 Mill. Euro, die einzige in Europa in Mathematik, die 2004 vergeben wurde, und der START-Preis (1,2 Mill. Euro), der einzige in Mathematik in Österreich 2004.

In den letzten sechs Jahren stellte der Fachbereich fünfmal den Förderungspreisträger der Österreichischen Mathematischen Gesellschaft (höchste Auszeichnung in einem Jahr an eine/n Mathematiker/Mathematikerin unter 40 Jahren).

Aussagekräftig ist auch die große Zahl an Wegberufungen an renommierte ausländische Universitäten und zusätzlich Rufe an ehemalige Institutsmitglieder bzw. AbsolventInnen an Spitzenuniversitäten, z.B. Harvard, Heidelberg, London, Paris, Berlin (Max-Planck-Direktor und Max-Planck-Förderungspreis 2004). 


\section{Teil B: Lehre und Studium an der Fakultät für Mathematik der Universität Wien}

Seit dem 1.10.2004 gibt es die Fakultät für Mathematik im Zusammenschluss mit dem Institut für Formale Logik (nunmehr Gödel Research Center).

\section{Ausbildungsprofil}

Die Fakultät für Mathematik der Universität Wien bietet neben dem Diplom- und Doktoratsstudium für Mathematik auch das Lehramtsstudium für Mathematik.

Durch eine Reihe von Abendvorlesungen werden auch für Berufstätige gute Möglichkeiten geboten.

Seit 2000 bietet die Fakultät für Mathematik der Universität Wien zusammen mit der Technischen Universität Wien und der Fakultät für Wirtschaftswissenschaften und Informatik den Lehramtsstudiengang Informatik im 1. oder 2. Hauptfach an.

Der Unterschied zwischen 1. und 2. Hauptfach beim Lehramt besteht darin, dass bei Wahl als 1. Hauptfach eine Diplomarbeit in Mathematik verfasst werden muss; beim 2. Hauptfach dagegen nicht. Des weiteren wurden bereits die Studienpläne für zwei Bakkalaureatstudien ("Angewandte Mathematik und Scientific Computing" und "Diskrete Mathematik und Computermathematik") erstellt. Die weitere Vorgehensweise im Bereich des Masterstudiums wird derzeit in der Studienkonferenz intensiv diskutiert.

Der Fachbereich bietet zu Beginn jedes Studienjahres zusätzlich Brückenkurse als Einstiegshilfe an.

\section{Allgemeine Ziele}

Diplom Mathematik:

Das Studium der Mathematik soll mit mathematischen Denkweisen vertraut machen und dazu befähigen, mathematische Fragestellungen in Theorie und Praxis zu bearbeiten. Dieses Ziel wird auf dem Hintergrund verfolgt, dass Mathematik seit langem einen großen Einfluss auf Technik, Natur- und Wirtschaftswissenschaften hat, wobei ihr bei der Fortentwicklung dieser Disziplinen sogar eine wachsende Bedeutung zukommt. Insbesondere nimmt die Mathematik für die sich zunehmend auffächernden Einzeldisziplinen eine integrierende Funktion wahr.

\section{Lehramt Mathematik:}

Die Lehramtsstudien der naturwissenschaftlichen und mathematischen Unterrichtsfächer dienen der fachlichen, fachdidaktischen und pädagogischen Berufsausbildung unter Einschluss einer schulpraktischen Ausbildung in zwei Unterrichtsfächern für das Lehramt an Höheren 
Schulen. Als Fachleute für die Vermittlung naturwissenschaftlich-mathematischer Kenntnisse erwerben die Absolventinnen und Absolventen auch Qualifikationen, die ihnen andere Berufsmöglichkeiten eröffnen.

\section{Lehramt Informatik:}

Das Studium des Lehramtes Informatik soll die AbsolventInnen für die folgenden vier Arbeitsbereiche qualifizieren:

- InformatiklehrerIn an Allgemeinbildenden Höheren Schulen (AHS)

- InformatiklehrerIn an Berufsbildenden Mittleren und Höheren Schulen (BMHS)

- InformatiklehrerIn im außerschulischen Bildungsbereich

- Informatikberufe außerhalb des Bildungsbereichs

Diese Berufsbilder erfordern nach der Vorstellung der Fakultät einerseits eine systematische Auseinandersetzung mit pädagogischen und fachdidaktischen Inhalten und andererseits Kompetenzen im fachlichen und wissenschaftlichen Bereich des Fachs Informatik.

\section{Praxisbezug}

Die Universität bietet ihren AbsolventInnen Kontakte zu Abnehmern über die informelle Ebene (Job-Börse auf der Homepage); im Gebiet Finanz- und Versicherungsmathematik z.B. sieht der wissenschaftlicher Verein INFORM, der am Institut beheimatet ist, in engerer Kooperation mit der TU Wien es als seine wichtigste Aufgabe an, den Kontakt mit Banken, Versicherungen und Finanzdienstleistern zu fördern.

Durch vielfältige Kontakte zu Vertretern der Wirtschaft und die am Institut durchgeführte Studie "Mathematisches Know-how in Österreich" wurde erkennbar, dass die AbsolventInnen in der Wirtschaft gerade wegen ihrer Vielseitigkeit, Kreativität und allgemeinen analytischen Fähigkeiten sehr gefragt sind. Entsprechend wurde bei der Konzeption der Studienpläne auf breite und fundierte mathematische Ausbildung Wert gelegt, die durch die forschungsbezogene Lehre gewährleistet ist. Die Anwendung mathematischer Software sowie allgemeine Computerkenntnisse werden beginnend mit den Anfänger-Proseminaren kontinuierlich vermittelt; die Möglichkeit zur Vertiefung ist durch ein (in Abhängigkeit von der budgetären Bedeckbarkeit) reiches Angebot an praxisorientierte Lehrveranstaltungen gegeben.

Die Studiengänge der Lehramtsstudien wurden gemeinsam mit den Fachdidaktikern des Instituts, die sämtlich einschlägige Schulunterrichtserfahrung besitzen, erstellt.

Aufgrund der Budgetknappheit sind Lehrveranstaltungen von Praktikern nach Aussage der Fakultät derzeit kaum möglich, obwohl seitens der Studierenden großes Interesse danach be- 
steht. Ein teilzeitbeschäftigter Finanzmathematiker, der auch in der Wirtschaft tätig ist und ein durch Drittmittel bezahlter Computerexperte ergänzen das Lehrangebot. Die schulmathematischen Lehrveranstaltungen im Lehramtsstudiengang werden stets von (ehemaligen) LehrerInnen abgehalten.

Am Institut sind regelmäßig GastprofessorInnen angestellt, die durch öffentliche Gelder bezahlt werden. Das Lehrangebot wird auch durch Gäste des ESI (Senior Fellows) und WPI bereichert. Eine verstärkte Einbindung von Lehrveranstaltungen dieser Gäste in das reguläre Studium ist derzeit im Aufbau begriffen. An den internationalen Programmen nehmen pro Jahr 10-15 Studierende teil.

\section{Personelle Ressourcen}

Die Lehrkapazität des festen Lehrkörpers (11 ProfessorInnen, 22 DozentInnen, 11 AssistentInnen) liegen zwischen 185 und 329 SWS. Das tatsächliche Lehrangebot der letzten 5 Jahre lag zwischen 287 Min. (2001) und 346 Max. (2004) SWS.

Aufgrund der angespannten finanziellen Situation gibt es mit Stichtag 1.7.2004 nur drei Lehrbeauftragte (zwei halten Informatiklehrveranstaltungen, einer Analysis Lehrveranstaltungen). Zusätzlich sind seit langem zwei BundeslehrerInnen (eine voll, eine halb) über Haushaltsmittel am Institut beschäftigt, die didaktische bzw. schulmathematische Lehrveranstaltungen abhalten.

Es werden regelmäßig als Serviceleistungen die Vorlesungen "Analysis für Physik und verwandte Gebiete" (Geophysik, Astronomie, Meteorologie) und "Lineare Algebra für Physik und verwandte Gebiete" mit den zugehörigen Proseminaren (insgesamt 24 SWS) abgehalten sowie die Vorlesung "Mathematische Grundlagen für das Physikstudium" mit Proseminar (6 SWS gemeinsam mit Physikern).

Die Standardvorlesungen, insbesondere die Anfängervorlesungen, werden auch von Studierenden anderer Institute resp. Universitäten besucht, z.B. Chemiker, Logiker, Statistiker, Informatiker der Technischen Universität.

Die Veranstaltungen für das Doktoratsstudium werden zu etwa 30 Prozent von (theoretischen) Physikern und Astronomen besucht.

\section{Sachausstattung}

Stand September 2004: 44 Arbeitsplätze in 3 PC-Labors. Eine Aufstockung der vorhandenen PC Labors um weitere 24 Arbeitsplätze wurde inzwischen genehmigt. Damit ist der Stand im Dezember 2004: 68 Arbeitsplätze in den 3 PC-Labors. 
Die Ausstattung der Bibliothek ist bei Standardtiteln und examensrelevanter Vertiefungsliteratur sehr gut. Sowohl die Hauptbibliothek der Universität Wien als auch die Fachbibliothek für Mathematik und Statistik besitzen Lehrbuchsammlungen für Mathematik mit Mehrfachexemplaren.

Weiterführende bzw. Forschungsliteratur findet sich an der Fachbibliothek, die sich im selben Gebäude wie das Institut befindet. Der Bestand ist diesbezüglich im Großen und Ganzen gut, vor allem deshalb, weil die Institutsmitglieder der Bibliothek eine Vielzahl von Rezensionsexemplaren schenken.

Im Bereich der Zeitschriften ist die Situation schwieriger. Die Bibliothek erhält viele Journale im Austausch für die Monatshefte für Mathematik, deren geschäftsführende Herausgabe im Institut liegt. Dadurch sind die entsprechenden Zeitschriften nur in gedruckter Form und nicht online verfügbar. In den letzten Jahren wurde der Bezug einiger wichtiger mathematischer Zeitschriften (z.B. Studia Mathematica, Mathematische Nachrichten, Journal of Analysis and Mathematical Applications) aufgrund der Budgetknappheit eingestellt.

Sämtliche Bibliothekskataloge sind online. Auch Feststellung von Verfügbarkeit und Reservierungen sind online möglich. Breiterer Online-Zugriff auf Zeitschriften wäre aus Sicht der Fakultät sehr wünschenswert, scheint aber aus budgetären Gründen kaum realisierbar.

Die Fakultät sieht Probleme in der räumlichen Ausstattung durch neue GastprofessorInnen und neue Forschungsstellen. Die finanzielle Situation ist momentan nur dadurch ausreichend, dass 50\% des notwendigen Budgets als Drittmittel eingeworben werden konnte.

Die Ausstattung mit PCs ist nach Ansicht der Fakultät verbesserungswürdig. In allerletzter Zeit konnten aber Verbesserungen erreicht werden.

Studienanfänger $(w / m)$

\begin{tabular}{|l|r|r|r|r|r|}
\hline Studiengang/ & 1999 & 2000 & 2001 & 2002 & 2003 \\
angestrebter Abschluss & & & & & \\
\hline Lehramt Mathematik & 177 & 232 & 158 & 143 & 209 \\
Lehramt Informatik & k.A. & 91 & 85 & 78 & 66 \\
Mathematik Diplom & 115 & 180 & 122 & 163 & 168 \\
Logik & 0 & 0 & 0 & 0 & 0 \\
\hline
\end{tabular}

Es wechseln immer wieder Studierende von anderen Universitäten nach Wien und zwischen Technischer Universität und Universität Wien. Die genauen Zahlen konnten vom Zentralen Informatikdienst der Universität Wien, Abteilung Universitätsverwaltung nicht glaubwürdig ermittelt werden. Aufgrund der Anträge auf Anrechnungen auswärtiger Studien schätzt die 
Fakultät die Zugänge auf 50-60/Jahr (etwa gleich viele Anrechnungen für Diplom wie für Lehramt).

Das durchschnittliche Alter der Studienanfänger Diplom und Lehramtsstudium in den letzten

5 Jahren betrug ungefähr 19,4 Jahre. Das Alter der Doktoranden ca. 26,6 Jahre

Studierende nach Studiengängen/angestrebten Abschlüssen und Fachsemestern

\begin{tabular}{|l|r|r|r|r|r|r|r|r|r|r|}
\hline Studiengang & \multicolumn{9}{|l|}{ Studierende im .......Fachsemester } \\
\cline { 2 - 8 } & 1. & 2. & 3. & 4. & $\cdots$ & 14. & 15. & 16. & 17. & $18 .+$ \\
\hline Lehramt Mathematik & 73 & 136 & & & & & & & & \\
Lehramt Informatik & 23 & 43 & & & & & & & & \\
Diplom Mathematik & 60 & 108 & & & & & & & & \\
Logik & & 22 & & 31 & & & & & & \\
\hline
\end{tabular}

Gesamtzahl der Studierenden:

- Lehramt Mathematik: 980

- Lehramt Informatik: 272

- Diplom Mathematik: 566

- Logik: $106(2 .-10$ Semester)

Studien- und Prüfungsorganisation

Zu jeder Hauptvorlesung werden Proseminare angeboten. In den ersten Semestern gibt es umfangreiche zusätzlich Tutorien und Repetitorien. In der Einführungsphase werden spezielle Workshops zur Aufarbeitung des Schulstoffes angeboten, die zu einem erheblichen Teil nicht von der Universität finanziert werden.

Lehramt: Der 1. Studienabschnitt besteht überwiegend aus Pflichtfächern. Im 2. Abschnitt sind ungefähr 2/3 Pflichtfächer, 1/3 Wahlpflichtfächer vorgesehen. Zusätzlich sind 10 SWS Freie Wahlfächer vorgeschrieben.

Diplom: Der 1. Studienabschnitt beinhaltet überwiegend Pflichtfächer.

Der 2. Studienabschnitt besteht etwa zur Hälfte aus Pflichtfächern und zur Hälfte aus Wahlpflichtfächern. Dabei gibt es Studienschwerpunkte, aus denen letztere zu wählen wären. Zusätzlich sind 12 SWS Freie Wahlfächer vorgeschrieben. 
Doktorat: Eine Vereinbarung der zu absolvierenden Lehrveranstaltungen geschieht mit dem/der Betreuer/Betreuerin.

Es gibt keine fest vorgeschriebene Abfolge der Lehrveranstaltungen, jedoch ist im Studienplan jeweils ein empfohlener Zeit- und Abfolgeplan inkludiert.

Parallelveranstaltungen werden abgehalten sofern budgetär möglich; des weiteren werden jährlich aufeinanderfolgende Lehrveranstaltungen stets von verschiedenen Dozenten angeboten. Die Anfängerproseminare werden teils computergestützt abgehalten. Die Professoren bestreiten etwa ein Viertel des Lehrangebots, der Mittelbau etwa drei Viertel. Die Anfängervorlesungen werden mehrheitlich von Professoren gehalten; Übungen dazu, die Einführungsphase sowie die Abendvorlesungen für Anfänger erfolgen fast ausschließlich durch den Mittelbau.

\section{Prüfungsverfahren}

Die 1. Diplomprüfung wird durch eine Vielzahl von Einzelprüfungen absolviert; hier gibt es keine Wiederholung der Diplomprüfung.

Bei der 2. Diplomprüfung liegt die Wiederholungsquote bei 10 Prozent.

Mindestens 3 Termine sind gesetzlich vorgeschrieben und werden auch angeboten. Der Prüfer ist üblicherweise der Leiter der Lehrveranstaltung. Bei Diplomprüfungen besteht freie Prüferwahl, wobei der Erstprüfer/die Erstprüferin der/die Betreuer/in der Diplomarbeit ist. Entsprechend wird bei Doktorprüfungen verfahren

Dauer der Abschlussarbeiten:

- Lehramt: 4 - 8 Monate Umfang 60 - 200 Seiten

- Diplom: 8 - 12 Monate 40 - 100 Seiten

- Doktorat: 12 - 24 Monate 40 - 100 Seiten

Die gesamte Prüfungsphase dauert im allgemeinen nur 2 - 4 Wochen, da die Diplomarbeit meist schon während der Erstellung der Letztfassung begutachtet wird.

Es gibt keine gesetzliche Mindestfrist bei Wiederholungen. Bei Einzelprüfungen sind im 1. Studienabschnitt 5, im 2. Studienabschnitt 4 Wiederholungen möglich. Bei Diplomprüfungen und Rigorosen sind 3 Wiederholungen erlaubt.

\section{Leistungsnachweise}

Die Leistungsnachweise in den einzelnen Disziplinen umfassen bei

- Lehramt Mathematik Hauptfach: 92 SWS 
- Diplom: 120 SWS

- Doktorat: weitere 12 SWS

- Lehramt Informatik: 90 SWS

- Logik: Studienabschnitt mit 63 SWS (Das Studium ist in der mit 6 Semestern vorgesehene Planstudienzeit studierbar).

Die Studiengebühren haben zu einer starken Zunahme des Anteils an Studierenden geführt, die zumindest teilweise berufstätig sind. Der Anteil von zumindest teilweise Berufstätigen liegt bei geschätzten $30 \%$.

Vollzeitstudierende schließen ihr Studium in der Regel rasch ab, im Gegensatz zu nebenberuflich Tätigen. Diese können fast nie ihr Studium in der Regelstudienzeit beenden, was den Entfall der staatlichen Familienbeihilfe bzw. von Stipendien zur Folge hat. Dadurch wird die finanzielle Situation dieser Studierenden oft prekär.

Durch das Abendstudium ist es aber auch für teilweise berufstätige Studierende möglich, wichtige Teile ihres Studiums neben ihrer beruflichen Tätigkeit ohne allzu große Zeitverlust $\mathrm{zu}$ absolvieren.

Durch diese Situation erklärt sich die bei einem nicht zu vernachlässigbaren Anteil von Studierenden deutliche Differenz zwischen Mindest- und Durchschnittsstudiendauer.

\section{Studienerfolg}

Im Zeitraum 1999 - 2003 wurden folgende 1. Diplomprüfungen abgelegt:

- Diplom: 141

- LA Informatik: 45

- LA Mathematik: 274

Die Zahl der AbsolventInnen betrug in den letzten 5 Jahren:

- Diplom: 76

- LA Mathematik: 275

- LA Informatik: 2 (Das LA Informatik wurde erst mit Wintersemester 2000 eingeführt).

- Doktorat: 32

- Logistik, Mag.phil: 1

Das durchschnittliche Alter der AbsolventInnen betrug:

Lehramt: 26 Jahre, Diplom: 26,7 Jahre, Doktorat: 29,8 Jahre, Logistik: 25 Jahre.

Die Fachstudiendauer bis zum Abschluss der 1. Diplomprüfung betrug:

- Lehramt: ca 2,8 Jahre 
- Diplom: ca. 3,2 Jahre

Die Fachstudiendauer bis zum Abschluss des Examens betrug:

- Lehramt: ca. 6 Jahre

- Diplom: ca. 7,2 Jahre

\section{Qualitätssicherung}

Die Qualitätssicherung wird durch Repetitorien und schon durch eine Einführungsphase mit Workshops zur Aufarbeitung des Schulstoffes sichergestellt.

Im zweiten Studienabschnitt gibt es anonyme unangekündigte Tests, mit denen der Beherrschungsgrad grundlegender Begriffe und Resultate überprüft wird, um allfällige Defizite wirksam bekämpfen zu können.

Lehrevaluation: Von Seiten der Universität Wien (Zentrum für Evaluation und Controlling) wird jedes dritte Semester eine verpflichtende Evaluation aller Lehrveranstaltungen durchgeführt. Daneben steht es den Lehrenden frei, diese Evaluation jedes Semester durchzuführen; von dieser Möglichkeit machen sehr viele Lehrende Gebrauch. Innerhalb von wenigen Wochen erhält jeder Lehrende die Ergebnisse für seine Lehrveranstaltungen. Dem Studienprogrammleiter (vormals Vorsitzenden der Studienkonferenz) werden sämtliche Ergebnisse der verpflichtenden Evaluation übermittelt.

Als Konsequenz der Ergebnisse wurden und werden Umschichtungen bei der Betrauung mit der Lehre vorgenommen. Davor gab es 15 Jahre lang eine freiwillige Evaluation über die Studierendenvertretung, die Ergebnisse wurden durch Aushang von derselben veröffentlicht. In der Studienkommission bzw. Studienkonferenz und in der Fakultätskonferenz wird regelmäßig zur Situation in der Lehre Bericht erstattet. Die Angebote zur Studieneingangsphase (Orientierungskurse u.ä.) werden regelmäßig hinsichtlich der gesammelten Erfahrungen überprüft und bei Bedarf erweitert, zuletzt durch die Einführung von spezifischen Workshops für AnfängerInnen. Die Studienpläne sind online verfügbar. Zu Beginn jeden Wintersemesters gibt es Informationsveranstaltungen und ein sehr detailliertes Merkblatt für StudentInnen. Für fortgeschrittene Studierende gibt es regelmäßige Informationsveranstaltungen als Orientierungshilfe für den Weg zum Abschluss des Studiums. Dabei werden auch mögliche Gebiete und Themen für Diplomarbeiten von den Betreuern vorgestellt

Als Defizit sieht die Fakultät, dass es durch die Vielfalt an Oberstufenformen sehr unterschiedliche Eingangsvoraussetzungen gibt. Es fehlt of an der Beherrschung von Lerntechniken, an Durchhaltevermögen und am sprachlichen Ausdruck. Es tragen auch falsche Vorstellungen der Studierenden über das Studium zum Abbruch bei. Diesen Defiziten wird durch Workshops, Repetitorien und verstärkten Einsatz von TutorInnen begegnet. Auch wird 
in der von der Fakultät maßgeblich gestalteten Lehrerfortbildung der ÖMG auf die LehrerInnen der Zubringerschulen eingewirkt. Weiter sind Mitglieder des Mathematischen Instituts als Lehrbuchautoren für die Oberstufe tätig, ein Mitglied ist in der Begutachterkommission für Schulbücher tätig und ein Mitglied leitet die Arbeitsgemeinschaft für MathematiklehrerInnen. Gesamteinschätzung der Fakultät

Die Stärken und Schwächen der Ausbildung werden von der Fakultät wie folgt beurteilt: Stärken:

- große Breite in der forschungsnahen Lehre

- weites Spektrum an Themen für Diplomarbeiten und Dissertationen

- Abendvorlesungen für Berufstätige

- Vernetzung von Lehramts- und Diplomstudium

- hohe Zahl von Lehramtsstudierenden

- Verzahnung von Fachwissenschaft und Fachdidaktik in der Lehramtsausbildung

- Verpflichtende Schwerpunktbildung im Diplomstudium

- Initiativen, um den AbsolventInnen bei der Berufswahl behilflich zu sein (Jobbörse, Informationsveranstaltungen).

Schwächen:

- $\quad$ in der Anfangsphase des Studiums zu wenig individuelle Betreuung möglich, vor allem wegen hoher Teilnehmerzahlen in Proseminaren

- Organisatorische Schwierigkeiten durch Wegberufung von Assistenten, die nach altem Dienstrecht angestellt waren, und die Einschränkungen des neuen Dienstrechts

- teilweise unterschiedliche Inhalte und Anforderungen in Grundvorlesungen. Mechanismen zur besseren Abstimmung sind derzeit in Planung

- Strukturbedingt wird Überblickswissen und Ausdrucksfähigkeit zu wenig gefördert und gefordert

Die Qualität der Ausbildung im Diplomstudiengang wird von der Fakultät als gut bis sehr gut bezeichnet, was auch der Erfolg der AbsolventInnen am Arbeitsmarkt zeigt. Die Qualität im Lehramtsstudiengang hingegen als zufriedenstellend bis gut. Problematisch ist hier, dass Mathematik häufig als zweites Fach gewählt und daher keine mathematische Diplomarbeit verfasst wird. Da MathematiklehrerInnen wesentlich gefragter sind als Lehrer der meisten anderen Fächer, wird im späteren Berufsleben dagegen hauptsächlich oder ausschließlich Mathematik unterrichtet. 
Es werden getrennte Grundvorlesungen in Analysis und Lineare Algebra angeboten. Dies führt schon in der Frühphase zu einer Betonung des deduktiven Charakters gegenüber einem mehr rezepthaften Zugang zur Mathematik, wie er bei solchen Ausbildungen vorherrscht, wo diese Trennung nicht vorgenommen ist (z.B. Mathematik I-IV Vorlesung).

Die hohe Drop out-Rate stellt für die Fakultät eine große Herausforderung dar, der sie seit einiger Zeit durch intensive Maßnahmen entgegenzusteuern sucht (Workshops, Studieneingangsphase, Tutorien, Repetitorien). Zum Teil kommt sie auch dadurch zustande, dass durch verstärkte Werbung in Schulen, Bildungsmessen, etc. sich offenbar auch InteressentInnen zum Mathematikstudium entschließen, die dafür nicht geeignet sind. Es wird versucht, die für das Studium ungeeigneten Studierenden möglichst früh aus dem Studienbetrieb auszuscheiden, um ihnen nicht die Möglichkeit eines rechtzeitigen Studienwechsels zu nehmen.

Die Fakultät sieht die Leistungsanforderungen für den ,normalen“ Studierenden im wesentlichen als angemessen an. Guten StudentInnen werden zusätzliche Anreize geboten. Engpässe im Lehrangebot gibt es derzeit keine.

Wünschenswerte Ergänzungen des Lehrangebots wären, den Praxisbezug zu erhalten und nicht durch weitere Kürzungen zu gefährden. Als weitere Veränderung wünscht sich die Fakultät die Einführung des Bakkalaureatstudiums wie im Bologna-Prozess gefordert, wobei die Beibehaltung der derzeitigen Studienpläne für Diplom- und Lehramtstudium bis zu dessen endgültiger Genehmigung gewünscht wird. Das Angebot des Doktoratsstudiums sollte weiter ausgebaut werden, wobei vor allem auf die Graduiertenkultur an der Fakultät besonderes Augenmerk gelegt werden sollte. 


\section{Empfehlungen}

\section{Eine erweiterte Rolle der Mathematik}

Alle Universitäten weltweit pflegen mathematische Wissenschaften in Forschung und Lehre. Obwohl Mathematik eine der ältesten Wissenschaften überhaupt ist, hat die mathematische Forschung an Dynamik nie nachgelassen. Während ihrer ganzen Geschichte wird sie getrieben durch innermathematische Fragestellungen, aber auch durch Probleme aus Natur- und Ingenieurwissenschaften. Seit jeher besteht eine besonders fruchtbare Wechselwirkung zwischen Mathematik und Physik. In neuerer Zeit sind lebenswissenschaftliche Bereiche wie Biologie und selbst geisteswissenschaftliche Fragestellungen hinzugekommen.

\subsection{Mathematik: Eine Wissenschaft mit Erneuerungspotential}

In den letzten Jahrzehnten ist die Wirkung der Mathematik bei der Lösung industrieller Probleme erheblich vielfältiger geworden. Selbstverständlich bedienen sich die traditionellen technischen Wissenschaften schon immer der Mathematik aber sie wirken auch ihrerseits in die Mathematik hinein, wie am Beispiel der Computerwissenschaften klar ersichtlich ist. Mehrere neu hinzukommende Fachbereiche nutzen mathematische Modelle: von der modernen Bankwirtschaft zur Fernmeldetechnik, von der Pharmaindustrie zur Sicherheitstechnik, vom Umweltschutz zur Luftfahrtindustrie.

Die damit verbundenen neuen Herausforderungen wirken auch auf die Mathematik indem sie eine permanente Reorganisierung des Faches verlangen mit neueren Beziehungen zwischen den Fächern, die früher wenig Berührungspunkte aufwiesen. Das gilt zum Beispiel für die neue Rolle, die stochastische Prozesse oder diskrete Mathematik spielen. In beiden Fällen existiert auf diesem Gebiet eine lange Tradition. Aber die neuen Wechselwirkungen mit anderen Fächern, die in den letzten Jahren entstanden sind, erweitern in gewaltiger Weise ihre Problemstellungen und verlangen originelle Zusammenführungen von verschiedenen Kenntnissen, die in unerwarteten Teilgebieten der Mathematik zu finden sind. Ein solcher Prozess ist besonders erkennbar in den sich schnell entwickelnden Feldern der Bio- und Nanotechnologie, wofür neue Rahmenkonzepte entwickelt werden müssen. Die wichtige Rolle, die heute die Statistik in so vielen verschiedenen Feldern spielt, hat in diesem Gebiet einen gewaltigen Forschungsbedarf zur Folge. Insbesondere muss die Verbindung mit anderen Fächern der Mathematik verbessert werden. Auch wichtige Fortschritte in der medizinischen Betreuung 
der Bevölkerung sind durch eine verbreitete Nutzung mathematischer Ressourcen zu erwarten. Moderne Techniken zur Einhaltung erhöhter Sicherheitsstandards beruhen auf kryptographischen datenkomprimierenden Algorithmen verschiedener Art. Aber alle habe ihr Fundamenten in hochentwickelten mathematischen Kenntnissen der algebraischen Geometrie oder der harmonischen Analysis, die früher nie mit diesem Ziel entwickelt wurden. Eine hervorragende Tradition existiert in diesen Gebieten in Österreich schon seit langer Zeit. Die Erfolge in Seismik und Tomographie, die auf Transformationen von Johan Radon beruhen, zeigen dies.

Der heute vielfach zitierte Satz „Hochtechnologie ist mathematische Technologie“ findet darin seine Begründung und signalisiert die neue Bedeutung und Dimension der mathematischen Forschung. Heutzutage ist Mathematik eine der wichtigsten Ressourcen für die Kompetitivität moderner Gesellschaften in der Wissenschaft und ihren Anwendungen. Das zeigt sich in der Nutzung von klassischen und neuen Resultaten, aber auch in den Ausbildungscurricula.

\subsection{Es besteht Handlungsbedarf: Mathematiker werden fehlen}

Tatsächlich erlebt heute die Mathematik einen besonderen Boom an spektakulären Forschungsergebnissen. Lange offene Probleme wurden gelöst, tiefliegende Fragen aus der Physik können mit neuen mathematischen Methoden erfolgreich und in wechselseitiger Befruchtung angegangen werden und der Bereich der Lebenswissenschaften stellt riesige Herausforderungen an die Mathematik.

Aber nicht nur die Forschung steht in einer hohen Blüte, verbunden damit ist auch die Entstehung neuer Arbeitsplätze für junge Mathematiker. Darauf muss die Ausbildung an den Hochschulen durch Ausgestaltung ihrer Curricula reagieren. Wir sehen heute, dass alle Studenten, die eine vertiefte Ausbildung in Mathematik erhalten haben, leicht eine Arbeitsstelle finden, aber auch dass die Zeit fast gekommen ist, in der Absolventen mit einer solchen Ausbildung fehlen werden. Einige Gesellschaften, wie EADS haben ihre Sorge darüber geäußert.

\section{Rahmenbedingungen}

Die Universitätsgesetze setzen Rahmenbedingungen innerhalb derer sich die Fächer entwickeln und in denen sich die Ausbildung des wissenschaftlichen Nachwuchses für Wissenschaft und Forschung sowie für die Wirtschaft realisieren lässt. 


\subsection{Unmittelbare Auswirkungen des neuen Universitätsgesetzes}

Der gegenwärtige Zustand der mathematischen Fachbereiche an den Universitäten Österreichs ist stark geprägt durch das Inkrafttreten des neuen Universitätsgesetzes. Die Auswirkungen der neuen gesetzlichen Regelungen bringen vor allem erhebliche Veränderungen für die Situation des wissenschaftlichen Personals mit sich. Das alte Universitätsgesetz hatte durch die Zementierung der Beschäftigungsverhältnisse auf der einen Seite und durch unklare Verantwortlichkeiten auf der anderen Seite zu einer Lähmung der Aktionsmöglichkeiten auf allen Leitungsebenen geführt. Aus dieser Sicht war die Ablösung der alten gesetzlichen Regelungen eine zwingende Notwendigkeit.

Um durch angemessene Veränderungen hin zu einem modernen, flexiblen und leistungsfähigen Hochschulsystem zu kommen, gibt es keine Patentrezepte. Eine Systemveränderung erfordert insbesondere in der Übergangszeit ein Vorgehen mit Augenmaß und unter Umständen vorübergehende Zugeständnisse in Form von Übergangsbestimmungen. So hat nach Meinung der Kommission die Stärkung der Universitäts- und Fakultätsleitungen zu einer Schwächung des Informationsflusses innerhalb der Hochschule geführt. Die Hochschulangehörigen fühlen sich nicht mehr ausreichend in die Entscheidungsprozesse eingebunden, was in einzelnen Fällen zu Resignation und innerer Emigration geführt hat.

\subsection{Das Personalproblem}

Die personelle Situation der Hochschulangehörigen im Mittelbau unter dem neuen Hochschulgesetz führt nach Meinung der Kommission zu einer Entwicklung, die vor allem in Hinblick auf den wissenschaftlichen Nachwuchs als bedenklich eingestuft werden muss. Eine geregelte Nachwuchsförderung scheint unter diesen Umständen kaum noch gewährleistet. Die Situation stellt sich gegenwärtig so dar, dass wissenschaftliche Mitarbeiter fast ausschließlich über Drittmittel finanziert und Universitätsstellen bei Freiwerden nur in Ausnahmefällen zur Wiederbesetzung frei gegeben werden. Davon sind vor allem die besten Universitäten des Landes betroffen, weil deren Nachwuchswissenschaftler an anderen Stellen attraktive Positionen finden. Die sich daraus ergebende und durchaus erwünschte Dynamik wird aber ins Gegenteil verkehrt, wenn die durch den Weggang entstandenen Lücken nicht wieder aufgefüllt werden können. In der Konsequenz stehen für den wissenschaftlichen Nachwuchs kaum attraktive Stellen zur Verfügung, so dass zu befürchten ist, dass in Kürze die österreichischen Universitäten ein echtes Nachwuchsproblem bei der Besetzung ihrer Stellen haben werden. Das Hauptaugenmerk der mathematischen Fachbereiche der Universitäten muss daher in $\mathrm{Zu}$ - 
kunft auf der Nachwuchspflege liegen, damit diese wesentliche Quelle der Erneuerung erhalten bleibt und weiter ausgestaltet werden kann.

\subsection{Eine gezielte Institutspolitik gegen die Vereinzelung}

Gegenwärtig ist zu beobachten, dass die Doktoranden an den Fachbereichen in der Regel zu alt sind. Darüber hinaus scheint jeder in einer gewissen Isolation vor sich hin zu arbeiten. Diese Vereinzelung muss beendet und durch perspektivische Strukturen im Mittelbau überwunden werden.

Die Kommission schlägt daher die Bildung von Zentren vor, in denen intensiver wissenschaftlicher Austausch stattfinden kann. Dafür gibt es bereits sehr gute Beispiele, wie die Einrichtungen der Universität Linz in Hagenberg mit dem RISC. Dort ist die Isolation der Doktoranden aufgehoben und es findet ein reger, auch internationaler Austausch statt. Dies hat die Kommission beeindruckt. Ein weiteres gutes Beispiel ist das Radon-Institut. Es gehörte allerdings nicht zum Auftrag an die Kommission, diese Spezialinstitute in die Evaluation mit einzubeziehen. Wegen ihrer Ausstrahlung auf die Universitäten werden sie hier nur kurz angesprochen. In diesen Fällen imponiert nämlich besonders die enge Verbindung zwischen universitärer Ausbildung und außeruniversitärer Forschung. Es ist hier auch gelungen, Zentren aus gesamtösterreichischer Sicht einzurichten, an denen mehrere Universitätsgruppen beteiligt sind. Obwohl diese Zentren teilweise noch im Aufbau sind, kann bereits jetzt gesagt werden, dass hier ein hoffnungsvoller Weg eingeschlagen wurde.

$\mathrm{Zu}$ den auch außerhalb Österreichs weithin bekannten Instituten gehört das ErwinSchrödinger-Institut, das ebenfalls nicht in die Evaluierung einbezogen war. Es hat sich für seine wissenschaftliche Leistungsfähigkeit bei effizienter Nutzung der zur Verfügung stehenden Mittel einen Namen gemacht. Das Erwin-Schrödinger-Institut steht in enger Verbindung mit der Mathematik der Universität Wien. 


\subsection{Der Stellenmarkt in der Mathematik}

Ein weiteres Problem scheint darin zu bestehen, dass für den wissenschaftlichen Nachwuchs in Österreich selbst nicht ausreichend Stellen zur Weiterqualifizierung zur Verfügung stehen. Der Stellenmarkt in Österreich selbst ist zu klein, um interessante Positionen für den wissenschaftlichen Nachwuchs in ausreichender Zahl zur Verfügung zu stellen. Allerdings ist für österreichische Wissenschaftler insbesondere der deutschsprachige Raum seit jeher als Stellenmarkt offen. Darüber hinaus hat sich inzwischen der europäische Markt für Wissenschaftler aus allen Ländern geöffnet. Die Kommission hat allerdings feststellen müssen, dass diese Positionen außerhalb Österreichs von den österreichischen Nachwuchswissenschaftler nicht im wünschenswerten Umfang angestrebt werden. Generell sollte darauf geachtet werden, dass wissenschaftliche Assistenten nach ihrer Promotion einen Wechsel der Hochschule vornehmen, wenn möglich durch Annahme oder zumindest Bewerbung auf entsprechende Positionen im Ausland. Der deutschsprachige Raum sowie die anderen europäischen Länder bieten sich da besonders an, ohne dass auf die liebgewonnenen Gewohnheiten im privaten Bereich verzichtet werden muss. Diese Art von Brain-drain wird nicht unbedingt als Problem gesehen. Er dient vielmehr der Öffnung der jungen österreichischen Mathematiker nach außen und dem späteren Reimport von Wissen und Internationalität bei der Rückkehr nach Österreich.

\subsection{Aufbau einer adäquaten Personalstruktur hat hohe Priorität}

Als zukünftige Personalstruktur sieht die Kommission zur Wahrung der Kontinuität die Lehrkanzeln als Dauerpositionen vor. Qualifikationsstellen sollten stets auf Zeit besetzt werden, wobei allerdings zu überlegen ist, ob nicht in geringer Zahl die Möglichkeit eines TenureTracks vorgesehen werden kann. Aufgrund des geringen Umfangs des österreichischen Stellenmarktes scheint es der Kommission ratsam, Dauerpositionen wie auch eventuelle Tenure Track-Stellen stets unter Rückgriff auf eine international zusammengesetzte Berufungskommission zu besetzen, um eine klare Qualitätskontrolle sicherzustellen. Die Gefahr der Bildung von Schulen, die sich auf Dauer etablieren, selbst dann, wenn die Aktualität der entsprechenden wissenschaftlichen Themen nachlässt, muss in Zukunft unbedingt vermieden werden. Die Kommission hält es für einen falschen und gefährlichen Weg, wenn in Zukunft Wissenschaftler nur noch auf temporären Stellen beschäftigt würden. Die Kontinuität würde darunter leiden, und die Verlässlichkeit der wissenschaftlichen Entwicklungen, auch gegenüber ausländischen Kollegen, würde zerstört. Ein System, in dem an der Spitze Lehrkanzeln stehen, die als Dauerposition besetzt werden, und darunter Qualifikationsstellen auf Zeit, die 
jedoch in einigen Fällen als Tenure-Track-Positionen ausgewiesen sind, erscheint der Kommission als ideal. Zur Qualifizierung des wissenschaftlichen Nachwuchses sollte ein Doktorandenprogramm, möglicherweise in Form von Graduiertenkollegs, aufgelegt werden, das nicht in zu enger Abhängigkeit zu existierenden Forschungsprogrammen ausgerichtet und verbunden ist. Auch mit dem Instrument der Juniorprofessuren wurden im Ausland bisher positive Erfahrungen gemacht. Es scheint auch in der Mathematik sinnvoll zu sein, solche Positionen des selbständigen Lehrens und Forschens, möglicherweise mit Tenure-Tracks versehen, einzurichten.

In der gegenwärtigen Situation des Übergangs von dem alten Universitätsgesetz zur neuen Regelung ist es erforderlich, die derzeit zu beobachtende Blockade und Perspektivlosigkeit für den wissenschaftlichen Mittelbau zu entspannen. Dazu könnten spezielle Sonderprogramme beitragen. Das Instrument der vorgezogenen Besetzungen hat sich hier in anderen Fällen bewährt. Bei klaren Vorstellungen über die zukünftige Entwicklung sollte den Fachbereichen die Möglichkeit gegeben werden, über vier bis fünf Jahre im Voraus vorhandene Lehrkanzeln zu besetzen. Das würde den Stellenmarkt entspannen und eine Perspektive auch für den wissenschaftlichen Nachwuchs schaffen. Es sei dazu nochmals betont, dass bei den entsprechenden Besetzungen auf internationale Berufungskommissionen zurückgegriffen werden müsste. Zur Besetzung solcher Stellen sollte möglichst ein breiter Konsens in den Fachbereichen erzielt werden. Das kann dadurch erreicht werden, dass in den Berufungskommissionen alle wissenschaftlichen Gruppierungen in geeigneter Form repräsentiert sind.

Die Lehrbelastung der Professuren unterschiedlichen Typs erscheint der Kommission sehr uneinheitlich geregelt. Nach unserem Eindruck gibt es Professoren, die sehr hohe Lehrbelastungen haben und andere, die wiederum zu der Lehre sehr wenige Semesterwochenstunden beitragen. Die Extrabezahlung von Überstunden erscheint uns kein geeignetes Mittel, um einen gerechten Ausgleich zu finden oder die wissenschaftliche Aktivität eines Institut oder einer Lehreinheit zu fördern.

Für eine an internationalen Standards orientierte österreichische Mathematik erscheint es sinnvoll, mit einer gewissen Regelmäßigkeit internationale Evaluationen durchzuführen, die sich nicht ständig auf ganz Österreich erstrecken müssen. Die entsprechenden Kommissionen sollten dazu überwiegend aus internationalen Experten bestehen. Etwa alle vier bis sechs Jahre scheint für solche Evaluationen ein geeigneter Zeitraum zu sein. Neben der damit doku- 
mentierten Ausrichtung an internationalen Standards kommt hinzu, dass so auch Informationen zwischen den verschiedenen Einrichtungen in Österreich wirkungsvoll transportiert werden könnten. Das wirkt der Vereinzelung und Isolierung der österreichischen Mathematik entgegen. Als oberstes Ziel sieht die Kommission, dass die Mathematik an österreichischen Universitäten so attraktiv wie möglich gestaltet werden muss, um für andere Länder sowohl auf Ebene der Studenten als auch auf Ebene des Lehrpersonals interessant zu sein. Die meisten österreichischen Universitäten sind hier auf einem guten Weg. Die Vergangenheit hat gezeigt, dass Österreich ein sehr interessantes Land für die mathematische Community ist, sobald es sich internationalen Entwicklungen öffnet. Auch im Hinblick auf die weitergehende Erweiterung der Europäischen Union in Richtung Osten kommt der österreichischen Wissenschaft sicher eine besondere Rolle zu. Verbünde und Absprachen mit Universitäten der Nachbarländer, die oft nur wenige Kilometer entfernt liegen, könnten zu einem interessanten Netzwerk führen. Das gilt für die Forschung wie für die Lehre, für die entsprechende Verträge geschlossen und Absprachen getroffen werden müssen.

\section{Zur künftigen Struktur der Hochschulmathematik in Österreich}

\subsection{Zahl der universitären mathematischen Ausbildungsstätten in Österreich}

Die derzeitigen Konzentrationsbemühungen in der Mathematik, wie sie z.B. an den beiden Grazer Hochschulen stattfinden, sind vor dem Hintergrund einer effizienten Nutzung vorhandener Potentiale sowie einer insgesamt erforderlichen verstärkten Kooperation der Hochschulen untereinander grundsätzlich zu begrüßen. Da aber an allen österreichischen Hochschulen im Fach Mathematik unterschiedliche Schwerpunkte bestehen, die eine weitere Profilierung erfahren, scheint es der Kommission nicht empfehlenswert, einen der Fachbereiche ganz zu schließen oder in andere Universitäten einzugliedern. Vielmehr sollte bei künftigen Planungen von den bisherigen Standorten des Faches Mathematik ausgegangen werden. Allerdings ist eine verstärkte Abstimmung der Forschungsfelder und Studienangebote zu einer homogenen Gesamtstruktur mit eindeutigen Prioritätensetzungen erforderlich. Zwar ist zu bemerken, dass einzelne mathematische Institute an den unterschiedlichen Hochschulen nur geringfügig ausgelastet sind, doch wird dieser Tatbestand häufig dadurch kompensiert, dass ein Übermaß an Serviceveranstaltungen für andere Fachbereiche geleistet werden muss. Insgesamt empfiehlt die Kommission, das Fach Mathematik an den einzelnen Einrichtungen zu stärken und weiter zu strukturieren. Die Ansätze hierzu sind an den österreichischen Universitäten teilweise schon sehr gut vorhanden. Nach Meinung der Kommission sollte das Fach Mathematik in 
seiner vollen Breite und mit internationaler Ausstrahlungskraft an der Universität Wien vertreten sein.

Die Technische Universität Wien hat, aufgrund ihres speziellen Ausbildungsprofils, vor allem in den Natur- und Technikwissenschaften wie auch in der Mathematik eine darauf ausgerichtete Profilierung zu erbringen. Mathematik sollte daher sowohl in der Forschung als auch in der Lehre an der Technischen Universität Wien stark anwendungsorientiert geprägt sein. Gleichwohl ist es erforderlich, dass die Basis für Angewandte Mathematik, nämlich Grundlagenforschung, auch an der Technischen Universität Wien vertreten ist.

Die Unterscheidung nach grundlagenorientierter und angewandter Mathematik entspricht ohnedies heute nicht mehr der Entwicklung des Faches. Mathematik ist ein Fach und es kommt vielmehr darauf an, Methoden der Problemlösung zu entwickeln und eine Gesprächskultur von Mathematikern und Nichtmathematikern zu pflegen. Darauf sollte die Technische Universität bei ihrer Neustrukturierung Wert legen. Die Kommission sieht in diesem Zusammenhang das derzeitige Vorgehen der Hochschule zur Umstrukturierung mit einer gewissen Sorge. Sie befürchtet, dass die jetzt eingeleiteten Prozesse einer nur schwer zu korrigierenden Schwächung des Fachbereiches führen werden.

Die mathematischen Fachbereiche an den beiden Grazer Hochschulen weisen in mancherlei Hinsicht ein ähnliches Profil auf. Die Aufgabenstellungen in der Lehre sind gleichwohl sehr verschieden. Um zu einer Konzentration der Aktivitäten in Graz zu kommen, ist es vorstellbar, dass trotz unterschiedlicher Aufgabenstellungen in der Lehre eine gemeinsame School of Mathematics in Graz gebildet werden könnte, in der vor allem die Forschungsaktivitäten abgestimmt werden und damit auch die internationale Sichtbarkeit noch stärker würde.

Der mathematische Fachbereich an der Universität Innsbruck erfüllt in seiner Struktur vor allem wichtige Aufgaben für die Ingenieurausbildung in Westtirol. Daher muss das fachliche Profil eine entsprechende Ausprägung besitzen. Trotzdem weist die Kommission darauf hin, dass auch hier als Nährboden für das Fach Mathematik in Forschung und Lehre eine hinreichende Stärkung der reinen Mathematik erforderlich ist.

Der Universität Salzburg steht für das sehr kleine Fach Mathematik eine nur eingeschränkte Vertretung des Fachs zur Verfügung. Die Kommission begrüßt daher, dass sich Salzburg im Wesentlichen auf die stochastischen Ausprägungen des Faches konzentriert hat. Trotzdem 
wird dringend empfohlen, bei zukünftigen Neubesetzungen die Grundlagen der Mathematik, die Voraussetzung für eine gute Lehre und Forschung auch in der Stochastik sind, nicht zu vernachlässigen, sondern zu stärken.

In ausgezeichneter Weise scheint es an der Universität Linz gelungen zu sein, ein eindeutig auch international sichtbares Profil zu erarbeiten. Die Ausrichtung auf Techno- und Industriemathematik hat nicht nur zur Profilbildung des Faches beigetragen, sondern auch bereits erhebliche Außenwirkungen in der Industrie und Wirtschaft erzielt. Auch die Grundlagen der Mathematik werden dabei nicht vernachlässigt.

\subsection{Anzahl der Fachrichtungen}

Wie in der Vergangenheit, so wird auch in Zukunft nicht an jeder Universität das Fach Mathematik in seiner gesamten Breite und in angemessener Qualität vertreten sein können. Diese Idealvorstellung ist nur auf der Ebene der gesamtösterreichischen Universitätslandschaft sinnvoll und kann nur im Zusammenwirken der Universitäten verwirklicht werden. Die Kommission empfiehlt daher, die Schwerpunktsetzungen und Konzentrationen in der Mathematik an den Universitäten so zu gestalten, dass bei sich gegenseitig ergänzenden Strukturen das gesamte Spektrum der Mathematik auf hohem Niveau in Österreich vertreten ist und eine sinnvolle Vernetzung auch über Österreich hinaus entstehen kann. Dies erfordert sicherlich die Bereitschaft einzelner Fachbereiche, zugunsten einer benachbarten Universität auf Entwicklungen gewisser Teilgebiete zu verzichten. Eine Konzentration auf Kernkompetenzen bei sinnvoller Weiterentwicklung ausgewählter Spezialgebiete ist hierfür Voraussetzung. Die vielfachen Serviceverpflichtungen des Faches Mathematik gegenüber anderen Fachbereichen dürfen nicht dazu verleiten, Mathematik an einzelnen Standorten nur noch als Serviceleistung anzubieten. Das würde die Entwicklung des Faches behindern und zu einer erheblichen Verminderung der Qualität beitragen. Grundsätzlich hält die Kommission an dem Prinzip fest, dass das Fach Mathematik nur von einem kräftigen und lebendigen mathematischen Fachbereich gelehrt werden sollte. Unberührt davon bleibt eine eventuelle schwerpunktmäßige Fokussierung auf Anwendungen der Mathematik. 


\subsection{Doppelangebote}

Angesichts unvermeidbarer Überschneidungen im Lehrangebot und daher in der curricularen Ausgestaltung von Studienzweigen insbesondere im Grundstudium können Doppelangebote in der Lehre nicht als zu beseitigendes Defizit betrachtet werden. Vielmehr ist in der Mathematik ein gewisser Grundstock an Lehrinhalten in jedem Fachbereich Voraussetzung für die Organisation und Durchführung der Lehre. Darüber hinaus sind Überschneidungen im Lehrangebot auch das Ergebnis von Diskussionen der Fachleute darüber, was ein Mathematiker in der Industrie oder in der Forschung können muss. Dazu besteht auch international ein Konsens.

Auch in der Forschung können und dürfen Überschneidungen in einzelnen Forschungsgebieten nicht immer unterbunden werden. In bestimmten Fällen sind Überschneidungen mit Blick auf die Steigerung der Leistungsfähigkeit einzelner Arbeitsgruppen durchaus wünschenswert. Echte Überschneidungen in Gegenstand, Methode und Zielsetzung einzelner Forschungsvorhaben sind selten, selbst hinter scheinbar gleichlautenden Disziplinen und Forschungsprojekten verbergen sich meist unterschiedliche Ansätze. Doppelungen deuten daher nicht generell auf einen unsachgemäßen und ineffizienten Einsatz von Forschungsmitteln hin. Vielmehr können sich durch parallele Forschungsvorhaben auch wertvolle Synergieeffekte entwickeln, die zugleich im Sinne eines wünschenswerten Wettbewerbs zwischen den mathematischen Fachbereichen verschiedener Hochschulen untereinander stimulierend wirken.

Die Kommission warnt daher davor, aufgrund oberflächlicher Betrachtungen der österreichischen Hochschulforschung in der Mathematik Kürzungen und Einsparungen vorzunehmen, die angebliche Doppelangebote abbauen sollen, häufig jedoch zur Zerstörung bewährter leistungsfähiger Strukturen führen. Hierdurch könnte sowohl die weitere Entwicklung des Faches auf Jahre hinaus beeinträchtigt werden.

\subsection{Standorte}

Die Standorte mathematischer Ausbildung und Forschung in Österreich erscheinen gut verteilt. Es besteht keine Notwendigkeit, weitere Standorte für die mathematische Ausbildung zu gründen. Insbesondere warnt die Kommission davor, die mathematische Ausbildung auch an Fachhochschulen anzusiedeln. Die sehr stark berufsbezogene Ausbildung an Fachhochschulen steht der generalisierenden Idee eines Mathematikstudiums entgegen. Andererseits zeigt 
das Beispiel der Universität Linz, dass durchaus im Rahmen eines klassischen Mathematikangebotes die Anwendungen und Anforderungen der österreichischen Industrie in der Ausbildung wie in der Forschung befriedigt werden können. Die Umstellung vom Diplom zum zweistufigen Studium Bachelor/Master wird hierfür noch mehr Gelegenheit geben.

Bei der Diskussion um die Profilbildung einzelner mathematischer Standorte erscheint es der Kommission wichtig, dass vor allem auf das Umfeld geachtet wird, in dem solche Angebote entstehen. Es ist sicher wenig sinnvoll, ein Angebot Finanzmathematik an einem Ort aufzubauen, an dem kein Finanzplatz vorhanden ist. Hier erscheint eine weitere Konzentration und Kooperation sinnvoll. In der Finanz- und Versicherungsmathematik könnte das etwa in Salzburg und Linz der Fall sein. Eine weitergehende Kooperation in der Finanzmathematik zwischen beiden Standorten wird daher angeregt. Es wurde bereits erwähnt, dass die Gründung einer School of Mathematics in Graz für die Forschung sinnvoll wäre. Aber auch in der Ausbildung, z.B. im CAD-Bereich, könnte unter Einsparung von Kapazitäten sinnvoll zusammengearbeitet werden.

Für derartige übergeordnete Strukturen gibt es eindrucksvolle Beispiele, etwa in Lausanne oder auch an den Berliner Fachbereichen. Ähnliche Überlegungen sind für die Universität Wien und die Technische Universität Wien nach Ansicht der Kommission wenig sinnvoll. Die Größe beider Hochschulen, aber auch die unterschiedliche Aufgabenstellung sprechen dagegen. Jeder Fachbereich hat für sich bereits ein sichtbares Profil auf internationaler Ebene erreicht. Das gilt auch für die TU Wien, vor allem mit Blick auf die für die Industrie relevanten Anwendungen.

\subsection{Universitäre und außeruniversitäre Forschung}

Die Kommission bestärkt die österreichischen Universitäten darin, das Fach Mathematik als ureigenen Bestandteil der Hochschulen schon aus der Historie heraus, aber auch im Blick auf die zukünftige Entwicklung der Wissenschaften beizubehalten. Die Einrichtung nur weniger außeruniversitärer Forschungseinrichtungen des Faches in enger Verbindung zu Universitäten wird besonders begrüßt. Darin sieht die Kommission einen besonderen Vorteil der österreichischen Mathematikerausbildung. 


\subsection{Zusammenarbeit von universitären und außeruniversitären Forschungseinrich- tungen}

Österreich verfügt z.B. mit dem Radon-Institut, dem RISC sowie dem Institut für wissensbasierte mathematische Systeme in Linz-Hagenberg über hervorragende und renommierte mathematische Forschungsinstitute, die in enger Verbindung zu den Universitäten stehen und eine große Ausstrahlungskraft auf alle mathematischen Fachbereiche des Landes und darüber hinaus haben. Die Kommission sieht darin eine hervorragende Möglichkeit, für die Mathematik internationale Standards zu setzen und eine Attraktion auch für nicht-österreichische Fachkollegen mit großem Renommee zu besitzen. Durch das geschickte Einbeziehen möglichst vieler österreichischer Universitäten in solche Zentren ist es gelungen, das Niveau dieses Faches auf ausgewählten Feldern beträchtlich zu erhöhen und sein Profil zu stärken.

Dieser Ansatz wird von der Kommission sehr begrüßt. Es erscheint für das Fach Mathematik jedoch nicht sinnvoll, weitere Institutionen dieser Art außerhalb von Universitäten zu gründen.

\section{4. Übergreifende Empfehlungen}

\subsection{Anzahl der Studienplätze}

Die Anzahl der Studienplätze sollte, obwohl nicht alle mathematischen Fachbereiche der österreichischen Universitäten voll ausgelastet sind, nach Meinung der Kommission nicht reduziert werden. Eine Reduzierung der derzeitigen Anzahl der mathematischen Studienplätze könnte im Widerspruch zur gesamtgesellschaftlichen Bedeutung dieses Faches stehen. Die Bemühungen Österreichs zur Verbesserung seiner industriellen Struktur setzen voraus, dass ausreichend viele Hochschulabsolventen im Hochtechnologiebereich verfügbar sind. Gerade die Absolventen der Mathematik spielen hierbei eine wichtige Rolle. Mathematische Kenntnisse werden als eine der Voraussetzungen zur Entwicklung von Hochtechnologien angesehen.

Nach Ansicht der Kommission wird Österreich auch im Hochschulbereich eine besondere Rolle im Rahmen der osteuropäischen Erweiterung der EU spielen. Die Randlage zu den neuen Beitrittsländern mit teilweise sehr renommierten mathematischen Ausbildungsstätten stellt eine große Chance der Kooperation für die österreichischen Hochschulen dar. Es ist denkbar, dass gerade dem Land Österreich auch im Fach Mathematik hier eine besondere Brückenfunktion zukommt. Die österreichischen mathematischen Fachbereiche sollten ermuntert wer- 
den, zu den benachbarten Hochschulen in Prag, in Budapest und anderen osteuropäischen Zentren intensiven Kontakt zu pflegen. Gemeinsame Internationale Graduiertenkollegs sowie gemeinsame Studiengänge oder zumindest eine Abstimmung von Studiengängen sind hierbei denkbar.

\subsection{Ausstattung der Fachbereiche}

Die Ausstattung der österreichischen mathematischen Fachbereiche mit Räumen und Computerinfrastruktur erscheint angemessen. Die derzeit eher fachlich orientierte Aufteilung von Räumen und Mitteln sollte sich jedoch stärker an leistungsorientierten Kriterien festmachen. Mit besonderer Sorge betrachtet die Kommission die Finanzknappheit und die damit verbundenen möglichen Kürzungen im Bibliotheksbereich. Zugleich weist sie darauf hin, dass die Hoffnung, im Rahmen einer verstärkten Nutzung elektronischer Medien die Kosten für den Bibliotheksunterhalt senken zu können, den bisherigen Erfahrungen nicht entspricht. Die Kommission verkennt nicht den häufigen Zwang zu Kürzungen und Einsparungen bei der Ausstattung der Fachbereiche, auch in der Mathematik. Vor allem bei der Vergabe entsprechender Mittel sollten Qualitäts- und Leistungsgesichtspunkte eine Rolle spielen. Die Kommission empfiehlt daher, die Vergabe von Personal- und Sachmitteln verstärkt an Leistungskriterien zu knüpfen. Hierdurch kann eine fiskalisch notwendige sowie unter Leistungsgesichtspunkten erforderliche Konzentration knapper Mittel auf aktive und engagierte Lehr- und Forschungseinheiten vorgenommen werden. Die Kommission empfiehlt den mathematischen Fachbereichen sowie den Universitätsleitungen, die jetzt bestehenden Spielräume zu einem derartigen flexiblen und gezielten Mitteleinsatz unter Leistungsgesichtspunkten auszuschöpfen. Zusagen sollten jedoch stets nur befristet vorgenommen werden. 


\subsection{Fakultäts- /Universitätsgrenzen}

Die Kommission empfiehlt, auch im Fach Mathematik die Möglichkeit zur fakultäts- und hochschulübergreifenden Zusammenarbeit in der Forschung in Zukunft noch stärker zu nutzen. Diese Empfehlung ist nicht nur vor dem Hintergrund einer effizienteren Entwicklung bestehender Forschungspotentiale zu sehen, sondern es besteht dabei auch die Chance, neuartige Ansätze in der Forschung zu verfolgen und zukunftsweisende Ergebnisse zu erzielen.

\subsection{Zusammenarbeit in der Lehre}

Nach Meinung der Kommission besteht noch erhebliches Potential zur verstärkten Kooperation auch in der Lehre an den einzelnen Hochschulen insbesondere dann, wenn mehrere Hochschulen am gleichen Ort vertreten sind. Beispiele wurden bereits für den Fall der beiden Grazer Universitäten genannt. Eine Abstimmung der jeweiligen Studienpläne hierzu scheint erforderlich. Es ist denkbar, dass gemeinsame Studienkommissionen gegründet werden könnten.

\subsection{Wahlfächer}

Die starke Reglementierung der Studiengänge im Fach Mathematik stellt für die Studierenden ein Hindernis dar, an Veranstaltungen ihrer Wahl teilzunehmen. Diese Situation könnte durch Gründung internationaler Graduate Schools aufgebrochen werden. Auch beim Angebot von Wahlpflichtveranstaltungen oder Wahlveranstaltungen scheint eine angemessene Kooperation der verschiedenen Hochschulstandorte möglich. Dazu sollten mit den Nachbaruniversitäten eine breite Palette von Wahlmöglichkeiten erarbeitet werden. Auf diese Weise kann ein insgesamt breites und attraktives Gesamtangebot entstehen, das von keinem mathematischen Fachbereich alleine in ähnlichem Umfang oder in vergleichbarer Qualität bereit gestellt werden könnte. Hierzu ist es aber möglicherweise erforderlich, entsprechende administrative Voraussetzungen für einen freien Zugang zu den Studienangeboten der Nachbaruniversitäten zu schaffen. Unter Nachbaruniversitäten sind hierbei auch solche Hochschulen zu verstehen, die zwar nicht in Österreich, aber in unmittelbarer Nachbarschaft österreichischer Universitäten liegen. 


\subsection{Prüfungsorganisation}

Der Kommission gegenüber wurde an allen mathematischen Ausbildungsstätten die Organisation der Lehre von den Studenten als gut bis sehr gut bezeichnet. Das Fach Mathematik, wie es an den jeweiligen Standorten angeboten wird, ist bei Konzentration auf das Studium auch in den dafür vorgesehenen Zeiträumen zu studieren. Jedoch erscheint der Kommission die Organisation der Prüfungen, und dabei insbesondere die häufige Wiederholbarkeit von Einzelprüfungen, nicht optimal. Hier sollte man sich an internationalen Standards ausrichten, die Wiederholungsmöglichkeiten für nicht bestandene Prüfungen in Einzelfächern deutlich reduzieren und damit das gesamte Prüfverfahren übersichtlicher machen.

\subsection{Lehrerausbildung}

Einer der großen Standorte der Lehrerausbildung, die Universität Klagenfurt, wurde von der Kommission nicht besucht. Daher konnte sie sich lediglich an den anderen Hochschulen ein Bild von der Qualität der entsprechenden Angebote machen. Die Kommission hatte insgesamt den Eindruck, dass an allen Standorten, an denen Mathematik als Lehramtsfach angeboten wird, dies mit großem Ernst und hoher Intensität geschieht. Auch die Arbeit der Hochschullehrer in der mathematischen didaktischen Ausbildung zeugt von großem Engagement, das in der Zusammenarbeit mit den Fachmathematikern besonders fruchtbar ist. Zweifellos erfolgt die Ausbildung in Österreich hier auf hohem Niveau. Bei der aktuellen Diskussion in Österreich um die Frage, ob mathematische Ausbildung für das Lehramt in Zukunft an pädagogischen Hochschulen angeboten werden soll, sieht die Kommission eindeutige Vorteile in einer universitären Ausbildung. Diese liegen vor allem in der breiteren und auch fachlich vertieften Ausbildung, die nur an den universitären Standorten gegeben ist. Mit Sorge hat die Kommission zur Kenntnis genommen, dass Mathematikstudenten im Lehramt in der Regel selten ihren Abschluss auch im Fach Mathematik machen. Sie weichen sehr häufig auf die anscheinend einfacheren Abschlussarbeiten in anderen Fächern aus. Dies kann auf längere Sicht zweifellos zu einer Minderung der Qualität in der Ausbildung von Mathematiklehrern führen. Die Kommission empfiehlt dringend, hier gegenzusteuern. Da die Kommission leider den Hauptausbildungsort für Mathematiklehrer, Klagenfurt, nicht besuchen konnte, sind die hier gemachten Anmerkungen möglicherweise nicht repräsentativ. 


\subsection{Wissenschaftlicher Nachwuchs}

Die Heranbildung eines qualifizierten wissenschaftlichen Nachwuchses ist eine der den Universitäten zugeschriebenen Hauptaufgaben. Nach Einschätzung der Kommission besteht jedoch die Gefahr, dass die österreichischen mathematischen Fachbereiche aufgrund der weitgehend auf Dauer besetzten Qualifikationsstellen nicht mehr in der Lage sein werden, diese Aufgabe angemessen zu erfüllen. Hierdurch wird letztlich auch ihre Leistungsfähigkeit in Forschung und Lehre beeinträchtigt. Ohne hinreichende Ausstattung der Hochschulen mit Qualifikationsstellen können weder neue Berufungen erfolgreich durchgeführt werden, noch können sich die mathematischen Fachbereiche im allgemeinen Wettbewerb um Drittmittel auch aus europäischen Quellen erfolgreich bewerben. Nach Auffassung der Kommission ist es daher dringend erforderlich, die bisherige Entwicklung zu korrigieren. Die Kommission hält Maßnahmen für erforderlich, die auch jetzt, in der Übergangsphase des neuen Hochschulgesetzes, dem wissenschaftlichen Nachwuchs ausreichend Chancen geben und letztlich zu einer ausgeglichen Personalstruktur führen. Es wird empfohlen, ein Programm zur Förderung des wissenschaftlichen Nachwuchses aufzulegen für den Zeitpunkt, an dem die derzeit auf Dauer besetzten Mittelbaustellen frei werden, um diese dann mit ausreichend zur Verfügung stehendem wissenschaftlichen Nachwuchs besetzen zu können. Dies könnte vor allem dadurch geschehen, dass international ausgerichtete Graduiertenkollegs gegründet werden, an denen der Vereinzelung der Doktoranden an österreichischen mathematischen Fachbereichen entgegen gewirkt werden könnte. Der Kommission ist aufgefallen, dass die Zahl der Doktoranden an einigen mathematischen Fachbereichen in Österreich sehr gering ist. Darüber hinaus scheinen die Doktoranden relativ isoliert zu arbeiten und in einigen Fällen auch nicht über die internationalen Entwicklungen des speziellen Faches orientiert zu sein. Es wird dringend geraten, die Internationalität der Doktorandenausbildung und der Weiterbildung nach erfolgter Promotion nachdrücklich zu fördern.

\subsection{Serviceleistungen}

Die Kommission ist der Auffassung, dass Querschnittsaufgaben und Serviceleistungen in der Mathematik für andere Fächer generell von mathematischen Fachbereichen wahrgenommen werden müssen. Isolierte Lehr- und Forschungseinheiten in fachfremden Fachbereichen verfügen in der Regel nicht über die kritische Masse, die für die anspruchsvolle und international anerkannte Forschung im Bereich der Mathematik nötig ist. Zudem unterliegen derartige Einheiten der Gefahr der Isolation innerhalb ihres mathematischen Heimatgebietes. Dies beein- 
trächtigt in der Regel die Qualität von Forschung und Lehre. Die Kommission empfiehlt daher mit Nachdruck einen mittelfristigen Abbau derartiger- in fachfernen Fachbereichen isolierter- mathematischer Einheiten und gegebenenfalls eine Reintegration in die Mathematik. Ausschlaggebend für diese Empfehlung ist neben Qualitätsgesichtspunkten auch das z.T. beträchtliche Einsparpotential, das durch die Konzentration von Serviceleistungen in einer Hand erschlossen werden kann. Detaillierte Hinweise finden sich in den Einzelempfehlungen zu den mathematischen Fachbereichen der betrachteten Universitäten.

\subsection{Altersstruktur}

In der Mathematik besteht eine zum Teil deutlich verzerrte Altersstruktur. Dies ist u.a. eine Folge des alten Hochschulgesetzes, durch dessen Auswirkungen habilitierte Mitarbeiter durchwegs eine Daueranstellung auf eigentlich als Qualifikationsstellen gedachten Positionen fanden. In den nächsten Jahren ist daher mit einer hohen Ausscheidequote der derzeitig auf Qualifikationsstellen sitzenden habilitierten Mitarbeiter zu rechnen. Auch aus diesem Grund hält es die Kommission für erforderlich, bei der Neubesetzung auf eine deutlich entzerrte Altersstruktur hinzuwirken. Dies könnte dadurch geschehen, dass man einen Teil der dann zu besetzenden Stellen für eine Vorbesetzung freigibt. Auf jeden Fall muss vermieden werden, dass in Forschung und Lehre mit dem gleichzeitigen Ausscheiden einer großen Zahl von Mitarbeitern ein Vakuum entsteht.

\subsection{Zentrale Serviceeinrichtungen}

Als zentrale Serviceeinrichtung für die Mathematik ist im Wesentlichen die informationstechnische Infrastruktur zu sehen. Um zu vermeiden, dass auf Qualifikationsstellen Dienstleister im Bereich der Informationstechnologie eingestellt werden, sollte in jedem Einzelfall geprüft werden, ob nicht die Kommunikationsinfrastruktur zentral bereitgestellt werden kann. Dies gilt insbesondere für den Bereich der großen Rechnerleistungen sowie für Zentraldienstleistungen. Es sollte vermieden werden, dass jede Arbeitsgruppe einen Mitarbeiter für den Service im Rechnerbereich bereitstellen muss. 


\subsection{Wirtschaftliche Komponenten mathematischer Studiengänge}

In Anbetracht der Tatsache, dass Mathematik immer mehr als Grundlagentechnologie für moderne Industrieentwicklungen angesehen wird, ist es erforderlich, dass sich mathematische Fachbereiche auch stärker der Erfordernissen der Wirtschaft öffnen. Die mathematischen Fachbereiche ihrerseits sollten darüber nachdenken, ob sie nicht verstärkt wirtschaftswissenschaftliche oder auch juristische Fächer als Nebenqualifikation in ihren Ausbildungskanon aufnehmen wollen. Der Studiengang Wirtschaftsmathematik hat sich bereits weltweit in diesem Zusammenhang etabliert. Neben solidem Fachwissen wird dabei auch wirtschaftswissenschaftliches Know-how vermittelt. Einige mathematische Fachbereiche an österreichischen Universitäten tragen dieser Entwicklung Rechnung und setzen sogar weltweit Standards. 


\section{Einzelempfehlungen: Zur weiteren Entwicklung der mathematischen Fachberei-} che

\subsection{Technische Universität Graz}

Die Technische Universität Graz verfügt in einzelnen Instituten über anerkannte Forschergruppen, die sich auch international einen Namen gemacht haben. Die Qualität der Forschung ist allerdings nicht überall auf dem gleichen hohen Niveau. Die Breite der Themen, die in einigen Instituten bearbeitet werden, ist eindrucksvoll. Jedoch könnten unter Umständen durch eine ausgeglichene Struktur der Institute noch stärkere Impulse gesetzt werden.

Nach Einschätzung der Kommission ist die Ausstattung der mathematischen Institute an der Technischen Universität Graz zufriedenstellend. Es gibt ausreichend gut ausgestattete Räume und eine gut bestückte Bibliothek. Das Reisebudget ist ausreichend. Die Struktur der einzelnen Mathematikinstitute, die nur nach ihrem Namen und den Anfangsbuchstaben des Alphabets geordnet sind, ist jedoch nicht klar verständlich.

Im Gegensatz zu früher scheint die Mathematik heutiger Ausrichtung sehr stark als Zulieferer für die Industrie zu dienen. Die Gutachter raten zu einer noch stärkeren Zusammenarbeit mit der Universität Graz, da es in vielen Bereichen thematische Überschneidungen gibt.

Die Technomathematik scheint nach Meinung der Kommission nicht sehr stark nachgefragt zu werden und wird auch nicht mit allem Ernst betrieben.

\section{Institut für Mathematik A (Analysis and Computational Number Theory)}

Im Institut für Mathematik A wird ein sehr breites Spektrum an Fragestellungen untersucht, jedoch fehlt den Arbeiten zum Teil die übergreifende konzeptionelle Tiefe.

Die Gruppe Arithmetic Diophantine Problems befasst sich mit einem breit gefächerten Spektrum an Fragestellungen zu speziellen diophantischen Gleichungen, Anwendungen des Schmidtschen Unterraumsatzes auf diophantische Gleichungen und lineare Rekursionen, diophantische N-Tupel sowie asymptotische Abschätzungen gewisser arithmetischer Funktio- 
nen und Formeln für die Anzahl von Gitterpunkten in gewissen konvexen Körpern. Die von den Gruppenleitern und ihren Mitarbeitern untersuchten Problemstellungen benötigen zur Lösung Ideenreichtum und raffinierte Kunstgriffe, da die Standardmethoden oft nicht zum Ziel führen. Andererseits fehlt den Fragestellungen z.T. eine übergreifende konzeptionelle Tiefe, so dass es in vielen Fällen weniger zu Publikationen in erstklassigen Zeitschriften kommt. Die Arbeitsgruppe hat viele Kontakte zu ausländischen Forschungsgruppen, z.B. zu Basel und Berlin. Umgekehrt wird die Gruppe von vielen Gästen besucht. Der Gruppenleiter ist Mitglied der österreichischen Akademie der Wissenschaften.

Eine Arbeitsgruppe im Institut für Mathematik A arbeitet sehr erfolgreich auf dem Grenzgebiet zwischen Algebra, Kombinatorik und Informatik. Die Themen reichen von der Verteilung von Binominalkoeffizienten in Restklassen über die gleichmäßige Verteilung von Punkten auf Splines bis zur Beschleunigung von Algorithmen, die in der Kryptographie eine Rolle spielen. Die Arbeit wird durch Publikationen gut dokumentiert und ist international anerkannt. Das belegen auch Einladungen zu einschlägigen Tagungen.

Das Institut für Mathematik A war in der letzten Zeit bei der Einwerbung von Drittmitteln besonders erfolgreich. Die Atmosphäre in diesem Institut macht einen sehr positiven Eindruck.

\section{Institut für Mathematik B (Mathematische Optimierung, dynamische Systeme und diskrete Mathematik)}

Der Leiter des Instituts B gehört zur ersten Generation der Vertreter der modernen, insbesondere diskreten mathematischen Optimierung. Seine Gruppe gilt als international führend bei der Identifizierung algebraischer Eigenschaften, die zu effizient lösbaren Spezialfällen des Rundreiseproblems, des quadratischen Zuordnungsproblems und anderer kombinatorischer Optimierungsprobleme führen. Erfolgreich werden auch immer wieder praktische Probleme etwa mit Methoden der ganzzahligen linearen Optimierung gelöst, wie beispielsweise Arbeiten zur Standortanalyse für einen international operierenden Konzern, zur Optimierung von Prozessabläufen in der chemischen sowie der Minimierung des Verschnitts bei diversen Herstellungsverfahren belegen. Zwei der insgesamt vier Mitarbeiter auf Assistentenstellen haben sich Themen außerhalb der mathematischen Optimierung zugewendet, so dass die eigentliche Gruppe derzeit bedauerlicherweise sehr klein ist. In der Vergangenheit hat diese Gruppe eine 
ganze Reihe an international als sehr stark erachteten Nachwuchswissenschaftlern hervorgebracht, die mittlerweile an Universitäten in Deutschland, Holland und Österreich tätig sind.

\section{Institut für Mathematik C (Mathematische Strukturtheorie)}

Im Institut für Mathematik $\mathrm{C}$ werden verschiedene Themen aus dem Gebiet der Graphentheorie bearbeitet. Der Schwerpunkt liegt auf einem Gebiet zwischen Stochastik und Angewandter Mathematik. In dieser Gruppe gibt es gute Einzelergebnisse.

Die Mathematische Statistik führt im Wesentlichen ein Eigenleben. Die Schnittstellen zur restlichen Mathematik der Technischen Universität Graz sind minimal. Es wird jedoch an guten Projekten gearbeitet. Leider entstehen wenig Publikationen.

Die Finanzmathematik sollte nach Einschätzung der Kommission an die Karl-FranzensUniversität Graz verlagert werden.

In der Geometrie werden ausschließlich CAD-Methoden angewandt, die dort nicht selbst entwickelt wurden. Die wissenschaftliche und auch didaktische Fundierung scheint zu fehlen. Die spezielle ausschließliche Ausrichtung auf die Industrie könnte genau so gut in den Ingenieurwissenschaften gelehrt werden. Hier sollte man darüber nachdenken, ob eine Professur, die auf moderne Geometrie ausgerichtet ist, eingerichtet werden kann. Die erforderlichen CAD-Methoden könnten auch von einem Mitarbeiter unterrichtet werden.

Die Kommission bewertet die neukonzipierten Bachelor- und Masterstudiengänge als sehr gut.

\section{Institut für Mathematik D (Numerik und Partielle Differentialgleichungen)}

Die Theorie der partiellen Differentialgleichungen ist z.Zt. an der Technischen Universität Graz nicht wirklich vertreten. Vereinzelt und mehr oder weniger unabhängig voneinander werden spezielle Probleme aus der Industrie behandelt. Diese Fragestellungen haben durchaus Relevanz und verlangen hohe Kompetenz. Es fehlt jedoch ein übergreifendes Forschungsund Lehrprogramm. Da eine personelle Erneuerung in Zukunft ansteht, sollte an eine Besetzung gedacht werden, durch die eine moderne Theorie der partiellen Differentialgleichungen gepaart mit konkreten Anwendungen implementiert wird. Der Forschungsgruppe Partielle 
Differentialgleichungen fehlt zur Zeit ein führender Kopf als Integrationsfigur. Bei der Wichtigkeit dieses Gebietes für eine Technische Universität muss hier dringend gehandelt werden.

In der Numerik gelang durch eine neuerliche Berufung die Gründung einer Keimzelle moderner numerischer Mathematik. Alternativ zur Besetzung einer analogen Stelle an der KarlFranzens-Universität hat der Stelleninhaber an der Technischen Universität seinen wissenschaftlichen Schwerpunkt bei Randintegral und Gebietszerlegungsmethoden. Eine enge Zusammenarbeit mit den fachlich nahestehenden Kollegen der Karl-Franzens-Universität wird dringend empfohlen. Es könnte ein Zentrum Numerik/Scientific Computing in Graz entstehen, das eine Ausstrahlung über Österreich hinaus entwickeln würde. Die personelle Kompetenz und die methodische Breite ist vorhanden.

\section{Institut für Geometrie}

Die Aktivitäten dieser Forschungsgruppe liegen in erster Linie auf Fragestellungen im Bereich Computer Aided Geometric Design und weiterhin auf konkreten Anwendungsprojekten wie z.B. der Steuerung von Schalungselementen beim Plattformbau und von Robotern. Ferner wurden in der Arbeitsgruppe interessante Beispiele von flexiblen Polyedern, einem klassischen Problem der Polyedertheorie, gefunden. In der Lehre nimmt die Arbeitsgruppe die Ausbildung zur darstellenden Geometrie für das Lehramt und die Ingenieurstudenten wahr. Die Technische Universität Graz ist neben der Technischen Universität Wien der einzige Ort in Österreich, an dem dies geleistet wird. Dazu erfolgt eine entsprechende Einführung in CAD-Softwaresysteme. In diesem Zusammenhang gibt es auch Untersuchungen über didaktische Probleme bei Gebrauch von CAD-Systemen.

\section{Institut für Statistik}

Die beiden Professoren dieses Instituts arbeiten in der angewandten bzw. der mathematischen Statistik. In den letzten Jahren wurden Probleme der angewandten Statistik und Datenanalyse bearbeitet. Dazu gehört z.B. die Anwendung der Theorie verallgemeinerter Mediane. Es wurden eine Reihe von erfolgreichen Kooperationsprojekten mit Partnern aus der Industrie und Verwaltung zu Fragen der Schaltkreissimulation bzw. der Prognose von Schadstoffaufkommen durchgeführt. Des weiteren wird an der klassischen Theorie der ungarischen Schule der Wahrscheinlichkeitstheorie über Fragen in der asymptotischen Verteilung von Statistiken und damit zusammenhängenden starken stochastischen Approximationen gearbeitet. In den letzten 
Jahren konnten gute Erfolge erzielt werden bei der Konvergenz und asymptotischen Verteilung von Schätzern bei Zeitreihen sowie bei log-linearen Modellen. Die Arbeiten sind in den einschlägigen Zeitschriften der Stochastik erschienen. Weiterhin hat das Institut mit seiner Arbeit über klassische Gitterprobleme in der analytischen Zahlentheorie auf sich aufmerksam gemacht. Zu harten offenen Problemen dieses Gebietes ist eine Reihe von guten Ergebnissen erzielt worden, die auch in den führenden einschlägigen Zeitschriften dieses Gebietes publiziert wurden. 


\subsection{Karl-Franzens-Universität Graz}

Die Kommission bestätigt die gute und übersichtliche Strukturierung des Instituts und eine sehr gute Bibliotheksausstattung. Auch die Sach- und Raumausstattung lässt keine Wünsche offen. Es fällt aber auf, dass die Forschungsgruppen stark von einer hierarchischen Struktur geprägt sind. Dies könnte damit zusammenhängen, dass die promovierten Mitarbeiter nach der Habilitation bisher in einem Dauerverhältnis unter Beibehaltung der Abhängigkeiten übernommen wurden, ohne etwa im Ausland Erfahrungen zu sammeln. Dadurch fällt es ihnen sehr viel schwerer, ein eigenes Profil zu entwickeln.

Die Kommission erinnert nochmals daran, dass nach ihrer Auffassung MathematikVorlesungen für andere Fachbereiche, hier die Physik, von Mathematikern des Fachbereichs Mathematik gehalten werden sollten. Das ist an der Karl-Franzens-Universität gegenwärtig nicht der Fall.

\section{Algebra- und Zahlentheorie}

In der Forschung hat sich die Arbeitsgruppe in den letzten Jahren erfolgreich damit beschäftigt, die Nichteindeutigkeit von Primfaktorisierungen in Integritätsbereichen (z.B. Ganzheitsringen) mit der Struktur von Monoiden in Verbindung zu bringen und diese wiederum mit Fragen über Nullsummen in der additiven Zahlentheorie zu verknüpfen, was unter anderem zu interessanten Anwendungen in der analytischen Zahlentheorie geführt hat. Dazu kommen Untersuchungen über die Endlichkeit bzw. Unendlichkeit der Anzahl von Lösungen gewisser Familien von Thue-Gleichungen sowie entsprechende Fragen im Funktionenkörperfall. Dies ist durch regelmäßige Publikationen der Arbeitsgruppe in anerkannten Zeitschriften und durch Buchpublikationen dokumentiert. In der Arbeitsgruppe finden regelmäßig Promotionen statt und ihre internationalen Kontakte sind gut.

Die Arbeitsgruppe zeigt sich in ihrer Forschungsarbeit aktiv und aufgeschlossen, mit einer gegebenenfalls neu zu etablierenden Arbeitsgruppe im Bereich der reinen Mathematik eng zu kooperieren. Dieses Potential sollte im Zuge der Neubesetzung einer der beiden in der Analysis frei werdenden Professuren genutzt werden. Die neue Professur könnte z.B. die algebraisch-geometrische Richtung abdecken.

In der Lehre engagiert sich die Arbeitsgruppe zusammen mit einer abgeordneten Mittelschullehrerin stark in der Lehramtsausbildung. In dieser Tätigkeit sollte die Arbeitsgruppe von an- 
deren Teilen des Instituts noch stärker unterstützt werden, damit die Lehramtsausbildung zu einem nach außen sichtbaren Schwerpunkt der Aktivitäten des Instituts gemacht werden kann.

\section{Analysis}

Die Analysis-Gruppe beschäftigt sich mit Fragen der Funktionalgleichungen und der Iterationstheorie und ist in diesem Bereich sehr aktiv. Die Fragestellungen entstammen der Tradition der 60er Jahre, konkrete Probleme in einem abstrakten Raum zu stellen und dort in möglichst großer Allgemeinheit zu lösen. Die Gruppe ist in internationale Verbindungen zu Forschern, die heute noch an diesen Fragen interessiert sind, eingebunden. Eine der Anwendungen ist es, Splines durch multivariable Funktionalgleichungen zu charakterisieren. Einige dieser Probleme sind motiviert durch die Charakterisierung von bestimmten Gruppenaktionen.

Einer der Forscher dieser Gruppe beschäftigt sich mit Anwendungen der Mathematik in der Musiktheorie und hat eine Reihe origineller Resultate erzielt. In der Gruppe wird die Zeitschrift Aequationes Mathematicae herausgegeben. Mehrere Arbeiten der Gruppe sind auch in dieser Zeitschrift erschienen. Im Zuge der anstehenden Erneuerungen sollte diese Gruppe eine veränderte wissenschaftliche Ausrichtung erfahren.

\section{Kontrolltheorie, Optimierung und Wissenschaftliches Rechnen, Scientific Computing}

Die Arbeitsgruppe Kontrolltheorie, Optimierung und Wissenschaftliches Rechnen sowie Scientific Computing bearbeitet innerhalb der angewandten Analysis ein sehr breites Themenspektrum. Es reicht u.a. von Newton-Techniken über Mehrgitterverfahren und Formoptimierung bis zur optimalen Steuerung von Navier-Stokes-Gleichungen. Auf den ersten Blick erscheint ein solches Programm von Themen kaum mit dem erforderlichen Ernst in der nötigen Tiefe bearbeitbar zu sein. Bei einer ausführlichen und substantiellen Diskussion mit der Arbeitsgruppe und vor allem auch mit den jüngeren Mitarbeitern wurde bei der Kommission dieser Eindruck jedoch stark relativiert. Die Kommission war beeindruckt von der Fachkenntnis und dem begeisterten Engagement der Wissenschaftler. Die sehr vielfältig erscheinenden Themen ranken sich um einen inhaltlich-methodisch einheitlichen Kern der mathematischen Optimierung und der numerischen Lösung der entsprechenden Probleme mit modernen Methoden. Dabei ist die numerische Lösung der Fragestellungen weniger auf algorithmische Realisierungen auf speziellen Computerarchitekturen mit dem entsprechenden Software- 
Engineering ausgerichtet, sondern mehr auf methodische Grundsatzfragen im Sinne einer Weiterentwicklung der klassischen Numerik. Die Arbeitsgruppe ist weltweit anerkannt. Ihre Arbeit ist durch gute Publikationen, Vorträge und Gasteinladungen dokumentiert. Sie sollte auch in dieser Breite und Ausrichtung weiterarbeiten. Daneben gibt es eine sehr kleine Arbeitsgruppe, die sich mit Visualisierungen in der Quantenmechanik befasst. Diese Arbeit ist zwar gut, aber randständig. Wenn der Bearbeiter ausscheidet, sollte diese Arbeit nicht weitergeführt werden.

Mit der Besetzung einer Vorziehprofessur wird die fächerübergreifende Plattform Scientific Computing eingerichtet. Dieses Vorhaben ist angesichts der immer größeren Bedeutung dieses Faches für alle Naturwissenschaften sinnvoll. Die neue Professur hat ihren Schwerpunkt in der Algorithmik, im Software-Engineering und der Computerarchitektur. Damit ist eine gute Ergänzung und Abrundung der Arbeiten in der angewandten Analysis gelungen. Um in Graz ein noch schlagkräftigeres Team im Scientific Computing zu etablieren, sollten entsprechende Bemühungen an der Technischen Universität Graz mit einbezogen werden.

\section{Modellierung und Dynamische Systeme}

Die Gruppe Modellierung und Dynamische Systeme wird von einem international sehr anerkannten Mathematiker geleitet. Die Forschungsarbeiten der Gruppe bewegen sich sowohl in der Reinen als auch in der Angewandten Mathematik. Die theoretischen Beiträge sind in der Funktionalanalysis angesiedelt und durch die Kontrolltheorie unendlich-dimensionaler dynamischer Systeme motiviert. Einen Überblick über die geleisteten Arbeiten kann man der kürzlich fertiggestellten Monographie „Evolution Equations and Approximations“ entnehmen. Weitere Beispiele der Arbeiten enthalten stochastische Aspekte der Halbgruppentheorie. Über die theoretische Forschung hinaus ist ein wichtiger Schwerpunkt der Arbeit dieser Gruppe die Anwendung der Theorie dynamischer Systeme in der Medizin, u.a. bei der Blutdruckstabilisierung und der Insulinregulierung. In diesem Bereich pflegt die Gruppe einen engen interdisziplinären Kontakt mit Medizinern und orientiert sich an der Fragestellung tatsächlich auftretender Probleme und nicht nur an der Anwendbarkeit bereits vorhandener mathematischer Theorien. Wichtige Aspekte dieser Arbeit liegen in der Modellbildung und in der quantitativen Identifikation von Parametern, die in diesen Gleichungen auftreten. Ein übergreifender Gesichtspunkt sowohl in der theoretischen als auch in der angewandten Forschung ist das Studium von Differentialgleichungen mit Verzögerung, das in dieser Gruppe durchgeführt wird. Im Bereich Mathematik und Medizin hat die Gruppe erfolgreich internati- 
onale Serien von Sommerschulen in Graz durchgeführt. Sie reicht bis zu Shape OptimizationProblemen und Freien Randwert-Aufgaben. Die Arbeitsgruppe Modellierung und dynamische Systeme genießt international einen sehr guten Ruf. Sie ergänzt das Spektrum auch anderer Arbeitsgruppen des Fachbereichs und sollte auf jeden Fall erhalten werden. 


\subsection{Universität Innsbruck}

Die Universität Innsbruck hat im Rahmen ihrer Neugliederung Institute, die thematisch verwandt sind, in eine neue Fakultät MIP (Mathematik, Informatik und Physik) zusammengefasst und an einem Standort konzentriert. Dies geschah teils um Mittel einzusparen, teils aber auch aus optischen Gründen, da aufgrund der stagnierenden Personalentwicklung und der geringen Fluktuation im Personalbereich, bedingt durch die vielen Dauerstellen, nur kleine Arbeitsgruppen existieren. Innsbruck spielt aufgrund seiner geographischen Lage für Westösterreich mit dem Einzugsbereich Tirol, Südtirol und Vorarlberg eine auch wirtschaftlich sehr wichtige Rolle. Es ist das geistige Zentrum für diesen Raum, von dem auch starke Impulse ausgehen.

Das Institut für Mathematik wurde erst vor kurzem zusammen mit dem Institut für Informatik in die Fakultät MIP verlagert. Die neue Struktur befindet sich noch in der Erprobungsphase, eine endgültige Festlegung ist bisher nicht getroffen. Das Institut für Mathematik existiert seit 1678 und war zeitweise in der philosophischen Fakultät angesiedelt. In den 70er Jahren hat auch hier der große Studentenboom zu einer starken Vermehrung von Stellen geführt, die mit jungen habilitierten wissenschaftlichen Mitarbeitern auf Dauer besetzt wurden. Das Institut war bis 1999 ausschließlich auf Reine Mathematik ausgerichtet, hat sich jedoch aufgrund der aktuellen politischen Vorstellungen und der starken Nachfrage der Wirtschaft nun mehr auf die Angewandte Mathematik spezialisiert.

In der Kommission besteht Konsens darüber, dass die starke Ausrichtung auf die Angewandte Mathematik als problematisch anzusehen ist. Die neuen Methoden auch der angewandten Mathematik haben ihre Wurzel in der reinen Mathematik, was auch vermittelt werden muss. Wegen des geringen Umfangs des Lehrkörpers wird allerdings keine Empfehlung ausgesprochen, die gesamte Breite der mathematischen Ausbildung anzubieten. Die Universität soll vielmehr ermuntert werden, ihre Stärken zu vertiefen, die in der starken Ausrichtung der Mathematik auf die Ingenieurausbildung liegen. Es sollte aber auf jeden Fall eine Professur in reiner Mathematik errichtet werden, damit eine gewisse Ausgewogenheit zwischen reiner und angewandter Mathematik gewahrt bleibt. Insbesondere erscheint die Umbenennung des Studiengangs in Diplom-Ingenieur für Technische Mathematik die Möglichkeit eines Abschlusses mit dem Schwerpunkt in Reiner Mathematik erheblich zu behindern. Dies kann auf lange Sicht der gesamten mathematischen Entwicklung in Innsbruck schaden. Um einer solch eher negativen Entwicklung gegenzusteuern, empfiehlt die Kommission die gezielte Neubesetzung 
von Professuren mit einigen zentralen und international aktiven Gebieten der Reinen Mathematik (z.B. Differentialgeometrie, Globale Analysis oder Algebraische Geometrie).

Die Lehrerbildung wird wegen der ausgezeichneten pädagogischen Ausbildung als sehr gut eingeschätzt. Die Gutachter sind sich einig, dass die Ausbildung der Lehrer an den Universitäten verbleiben und nicht an Pädagogische Hochschulen verlagert werden sollte, wie dies zurzeit in Österreich diskutiert wird. Es wäre gut, wenn für die Didaktik eine eigene Professur eingerichtet werden könnte. Das Doktorandenprogramm sollte auch für Lehramtsstudenten zugänglich gemacht werden.

Allgemein gewann die Kommission den Eindruck, dass ein Studium in Innsbruck zu stark an lokalen Interessen ausgerichtet ist. Die Mobilität der Absolventen ist wenig ausgeprägt. Das trifft auch auf deren Aufenthalte an Zentren der Mathematik wie Oberwolfach oder Luming zu. Junge exzellente Mathematiker benötigen solche Herausforderungen. Nach Aussage des Fachbereichs steht den Wissenschaftlern ausreichend Zeit für Forschung zur Verfügung. Das spiegelt sich nicht im wissenschaftlichen Output wieder.

\section{Institut für Mathematik}

\section{Algebraische Zahlentheorie und Galoistheorie}

Die Arbeitsgruppe Algebraische Zahlentheorie und Galoistheorie interessiert sich vor allem für Galois-Modulstrukturen und Explizite Kommutative Algebra. Die Veröffentlichungen enthalten sehr interessante Arbeiten. In der Gruppe arbeiten sehr gute Studenten und Doktoranden, die auch internationale Tagungen besuchen und sich an den Arbeiten im Ausland orientieren. Der Eindruck zu dieser Arbeitsgruppe ist sehr positiv. Diese relativ kleine Gruppe leistet sehr gute Arbeit.

\section{Dynamische Systeme und Funktionalgleichungen}

Die Gruppe ist sehr klein. In den letzten Jahren wurden relativ wenige Publikationen geschrieben. Diese beschäftigen sich mit abstrakten Funktionalgleichungen sowie geometrischen Problemen in der Ebene. In der Vergangenheit wurden einige gute Arbeiten über Intervallabbildungen und zugehörige symbolische Dynamiken verfasst. Schöne Ergebnisse existieren zur expliziten Bestimmung invarianter Masse und eine interessante Charakterisie- 
rung der topologischen Entropie. Obwohl auch aus neuerer Zeit einige Publikationen vorliegen, ist insgesamt noch zu wenig publiziert. Die Kommission hat den Eindruck, dass hier relativ isoliert mit wenigen internationalen Kontakten gearbeitet wird. Dieser Befund über die Isolation von Einzelforschern, deren Aktivität und Kreativität wegen fehlender lokaler, nationaler und internationaler Kontakte langsam versiegt, findet sich in einer Reihe der von der Kommission begutachteten Einrichtungen.

\section{Geschichte der Mathematik}

Diese Arbeitsgruppe besteht aus einem einzelnen Hochschullehrer. Er beschäftigt sich mit der historischen Aufarbeitung der mathematischen Curricula der international hoch anerkannten Mathematiker Gröbner und Vietoris, die über viele Jahre an der Universität Innsbruck gelehrt und geforscht haben. Von ihnen liegt ein großer Nachlass vor, der dem Institut übertragen wurde. Die Arbeiten des Hochschullehrers werden von der österreichischen Akademie der Wissenschaften unterstützt. Die Kommission betrachtet die Erforschung und Herausgabe der Werke von Gröbner und Vietoris als eine wichtige Aufgabe des Instituts. Es wird angeregt, ein umfassendes Konzept zur Herausgabe dieser Werke beispielsweise über ein SWF-Projekt $\mathrm{zu}$ erstellen und zu realisieren.

\section{Jordan-Theorie und Steinberg-Gruppen}

Auch diese Arbeitsgruppe ist sehr klein und wird von einem bereits emeritierten, allerdings sehr aktiven Hochschullehrer geleitet. Durch das hohe Ansehen des Leiters der Arbeitsgruppe verfügt die Gruppe über zahlreiche internationale Kontakte.

\section{Lineare Systemtheorie und Computeralgebra}

Hauptthema dieser Arbeitsgruppe ist die Multidimensionale Systemtheorie. Diese ist auf einer kategorischen Dualität zwischen algebraischen und analytischen Objekten aufgebaut. Die Ergebnisse dieser Gruppe erscheinen interessant. Die Forschung ist alles in allem jedoch eher randständig. 


\section{Institut für Technische Mathematik, Geometrie und Bauinformatik}

\section{Arbeitsgruppe Numerik von Evolutionsgleichungen}

Diese Arbeitsgruppe hat gute internationale Kooperationen. Sie bearbeitet aktuelle Forschungsfelder. Ihre Publikationen befinden sich auf hohem Niveau. Die Gruppe sollte vergröBert werden und mit Spitzenforschern aus der Numerik zusammenarbeiten, die auch praktische Methoden einsetzen.

\section{Arbeitsgruppe Nichtlineare Analysis/Unscharfe Daten}

Diese Gruppe ist sehr aktiv und arbeitet auf einem wichtigen Forschungsfeld. Auch diese Gruppe sollte vergrößert werden und mehr Kooperationen mit Spitzenforschern aus Forschung und Wirtschaft eingehen. Der Arbeitsgruppenleiter hat eine Reihe von Monographien und eine große Zahl von Arbeiten zur Theorie linearer partieller Differentialgleichungen mit singulären Koeffizienten bzw. singulären Anfangsdaten sowie stochastische Differentialgleichungen und nichtlineare gewöhnliche Differentialgleichungen auf der Basis der Colombeauschen Algebra verallgemeinerter Funktionen verfasst. Er hat diesen funktionalanalytischen Ansatz zur Definition nichtlinearer Funktionen von Distributionen auf zahlreiche Modelle mit Singularitäten, z.B. in der Relativitätstheorie, der Ausbreitung von Singularitäten und Schockwellen sowie Modelle der Quantenphysik angewandt. Erwähnt seien auch Arbeiten eines Mitarbeiters zur Begründung der Theorie stochastischer semilinearer partieller Differentialgleichungen. Eine Reihe von Schülern dieses Hochschullehrers arbeitet an verwandten Problemen an der Universität Wien. Ob diese Arbeiten, obwohl sie das Interesse der Ingenieure und Physiker finden, einen größeren Einfluss auf das sehr aktive Gebiet der nichtlinearen partiellen Differentialgleichungen gewinnen werden, ist nicht klar. Ein weiterer Mitarbeiter beschäftigt sich mit der Kombination von stochastischen Modellen mit unscharfen Parametern, die im Bauingenieurwesen zur Vorhersage von Zuverlässigkeitsschranken in Form von geordneten Mengen von Wahrscheinlichkeitsmaßen benutzt werden. Es bleibt unklar, welche Vorteile dieser Ansatz gegenüber der klassischen Modellvalidierung hat.

Es ist zu empfehlen, dass die Gruppe Nichtlineare Analysis/Unscharfe Daten mit einer neu einzurichtenden Gruppe Differential- oder Globale Geometrie zusammenarbeiten sollte, um das Umfeld insgesamt attraktiver zu machen. 


\section{Arbeitsgruppe Biomechanik}

Dies ist eine sehr kleine Gruppe, die auf einem für den österreichischen Standort wichtigen Forschungsgebiet arbeitet. Allerdings steht sie auch in Konkurrenz zur kommerziellen Spieleindustrie. Bei der geringen Größe der Gruppe ist es fraglich, ob sie diesem Konkurrenzdruck standhalten kann. Sie sollte möglicherweise einer anderen Gruppe angeschlossen werden.

\section{Arbeitsgruppe Geometrie/EBRA}

Die Arbeitsgruppe beschäftigt sich u.a. mit der Ein Bild Röntgen Analyse (EBRA) mit Anwendung in der medizinischen Forschung. Ein weiterer wichtiger Forschungsbereich dieser Gruppe liegt in der Kinematik und der Robotergeometrie mit Anwendung z.B. auf Flugzeugsimulatoren und dem Hexapod-Teleskop. Die mathematischen Methoden stammen aus der darstellenden Geometrie und reichen bis zur reellen algebraischen Geometrie. Die Gruppe macht einen aktiven und lebendigen Eindruck. Es gibt eine Vielzahl internationaler Beziehungen, z.B. mit der McGill University in Montreal (Kanada), sowie produktive Verbindungen mit der Industrie. In den letzten Jahren wurden mehrere Dissertationen in diesem Bereich abgeschlossen.

Es gibt Bestrebungen, die Gruppe InfMath Imaging in diese Arbeitsgruppe zu integrieren. Diese Bemühungen werden von der Kommission nachdrücklich unterstützt. Es werden bereits gemeinsame Seminare veranstaltet, und eine Zusammenlegung kann einer Weiterentwicklung beider Gruppen und der wissenschaftlichen Interaktion förderlich sein. Solche gegenseitigen Inspirationen sind fruchtbar für die wissenschaftliche Entwicklung. Mit der räumlichen $\mathrm{Zu}$ sammenlegung dieser beiden Gruppen würde die notwendige kritische Masse für eine positive Entwicklung entstehen.

\section{Institut für Informatik}

\section{Arbeitsgruppe InfMath Imaging}

Die Gruppe arbeitet auf hohem Niveau und bietet eine gute Kombination zwischen Mathematik und Computer Science. Sie ist gut organisiert und arbeitet mit nationalen und internationalen Kooperationspartnern. Die Ausstattung und das Ausbildungsmaterial werden als sehr gut eingeschätzt. Die Gruppe gibt eine eigene Printreihe heraus. Von der Gruppe InfMath Imaging können wichtige Impulse für die Mathematik und die Ingenieurwissenschaften.ausgehen 


\subsection{Universität Linz}

Die Universität Linz hat früh auf Schwerpunktbildung im Fachbereich Mathematik gesetzt und zusammen mit dem ersten Spezialsonderforschungsbereich Numerical and Symbolic Scientific Computing und dem Radon-Institut der Österreichischen Akademie der Wissenschaften ein Zentrum der angewandten Mathematik in Österreich gebildet, dessen Vision die Zusammenführung von symbolischen, numerischen und geometrischen Methoden des wissenschaftlichen Rechnens ist. Die drei Zentren arbeiten eng zusammen in dem Bemühen, Lösungen für großskalige, direkte und inverse Probleme mit Nebenbedingungen für Real-LifeProbleme von hoher Komplexität zu finden. Der Fachbereich Mathematik hat unter diesem Anspruch bereits eine Reihe von Spin-offs gegründet, die für österreichische Unternehmen Probleme lösen. Der Fachbereich Mathematik an der Johannes-Kepler-Universität Linz wurde 1968 gegründet und besteht heute - nach zwei gesetzlichen Umstrukturierungsmaßnahmen aus zehn mathematischen Instituten mit stark anwendungsorientierter und interdisziplinärer Grundlagenforschung mit teilweise direkter oder indirekter Umsetzung in Industrieprojekte. Die Institute sind untereinander stark vernetzt und arbeiten an gemeinsamen Publikationen, gemeinsamer Softwareentwicklung und gemeinsamen Forschungsprojekten. In der Evaluationskommission bestand Einigkeit darüber, dass Linz in seiner Strukturierung und Bildung von Kompetenzzentren eine gelungene Lösung zur Bewältigung der Situation der österreichischen Mathematik verfolgt. Die Atmosphäre unter den Mitarbeitern ist ausgesprochen gut, es ist eine große Dynamik zu spüren. Die mathematische Ausbildung ist breit angelegt, besonders das Angebot in Algebra ist sehr gut. Es gibt eine Reihe ausgezeichneter Wissenschaftler, die hervorragende Ergebnisse produzieren. Linz hat seine Nische gefunden, und durch die vielen Industriekooperationen und Forschungsprojekte sind auch genügend finanzielle Mittel vorhanden, um Postdocs/Doktoranden einzustellen, die in den Projekten mitarbeiten. Nachteilig könnte sich eventuell auswirken, dass die Studenten durch die intensive Projektarbeit nicht zu einem Wechsel an eine andere Universität bereit sind. Bei mangelnder Kompatibilität der Curricula könnte diese Tendenz noch verstärkt werden. Die Gutachter geben zu bedenken, dass bei allen wirtschaftlichen Erfolgen das Fundament, die Reine Mathematik, nicht aus den Augen verloren werden darf. Die von der Universität gewählte Institutsstruktur bietet Vorteile, da die Verantwortung für Verwaltung und Forschung bei den Instituten liegt und sie damit in einer flachen Hierarchie klar definiert geregelt ist. Die Gutachter geben jedoch zu bedenken, dass das System wahrscheinlich nur reibungslos funktioniert, solange die Institutsleiter gleichberechtigt nebeneinander arbeiten. Sollten hier Anzeichen für eine Veränderung erkennbar werden, muss rechtzeitig gegengesteuert werden. 


\section{Institut für Algebra}

Die Forschungsthemen dieses Instituts liegen in der Strukturtheorie von Fastringen, Polynomfunktionen und algorithmischen Aspekten. Die Veröffentlichungen haben ein hohes Niveau und sind international anerkannt. Es gibt zahlreiche Kontakte und eine intensive Zusammenarbeit mit anderen Instituten. Der Forschungsschwerpunkt liegt auf der Theorie, die Gruppe ist jedoch auch darüber hinaus an Anwendung interessiert. Sie nutzt jede Möglichkeit der Zusammenarbeit mit anderen Forschungsrichtungen. Die Gruppe vermittelt einen aktiven und dynamischen Eindruck.

\section{Institut für Analysis}

Im Institut für Analysis sind vor allem die Arbeiten zu funktional- und harmonischanalytischen Themenbereichen hervorzuheben. So haben die Arbeiten zur Lösung der Andersson-Vermutung oder die bemerkenswerte Vereinfachung einer Martingal-Ungleichung von J. Bourgain Aufsehen erregt. Obwohl diese Arbeiten herausstechen und zu vielen internationalen Kontakten mit renommierten Mathematikern beigetragen haben, ist der Bearbeiter dieser Problematik innerhalb Österreichs relativ isoliert. Seine Ergebnisse erscheinen regelmäßig in international führenden Zeitschriften.

Die Kommission ist der Meinung, dass im Zuge der Nachfolgeregelung des jetzigen Institutsleiters die Analysis an der Universität Linz mit einer international ausgewiesenen Persönlichkeit gestärkt wird, die einerseits die genannten Aktivitäten noch stärker fokussiert und andererseits als Ansprechpartner bei Grundlagenfragen in der Analysis für die angewandten Teile des Fachbereichs fungieren kann.

Zur Abteilung Analysis gehört auch der Bereich Finanzmathematik, der durch die jüngste Berufung aus Salzburg weiter gestärkt wurde und die Verbindung zu der Universität Salzburg auf diesem Gebiet weiter festigt. Die Finanzmathematik wird mit hohem mathematischen Anspruch hinsichtlich der Diplome und Dissertationen von Wissenschaftlern engagiert betrieben, die auf dem Gebiet der Diophantischen Approximationen und Diskrepanztheorie aktiv und gut ausgewiesen sind. Die Vorlesungen mit einer beachtlichen Zahl von Studierenden erfreuen sich lebhaften Interesses.

Eine weitere beachtenswerte Aktivität findet sich auf dem Gebiet der Dynamischen Systeme. Die Gruppe arbeitet erfolgreich zur Iteration polynomieller Abbildungen und zu Fragen der Approximationstheorie und Orthogonaler Polynome. Auch diese Arbeiten sind in führenden einschlägigen Zeitschriften erschienen. 


\section{Institut für Industriemathematik}

Der Institutsleiter ist eine äußerst aktive Person. Er hat Pionierarbeit in Österreich und Europa geleistet. Auch die Gruppe ist äußerst aktiv und produktiv und hat sehr gute Publikationen in großer Zahl vorzuweisen. Über den Institutsleiter verfügt die Gruppe über eine Reihe internationaler Kontakte und über exzellente nationale und internationale Verbindungen. Die Doktorandenausbildung ist gut. Verbindungen zur Industrie und die entsprechenden Aktivitäten haben den Fachbereich Mathematik in Linz entscheidend mitgeprägt.

\section{Institut für numerische Mathematik}

Das Institut arbeitet auf Weltklasseniveau. Die Infrastruktur ist ausgezeichnet, die Studienpläne und die Studienstruktur sind sehr gut organisiert und die Studenten werden sehr gut betreut. Die Ausbildung ist effizient organisiert, es gibt jedoch zu wenige Doktorstudenten. Die Gutachter empfehlen, ein nationales Doktorandenprogramm auch für Lehrer einzuführen und die Doktorarbeiten von jeweils zwei externen Gutachtern bewerten zu lassen.

\section{Institut für Stochastik}

Das Institut hat in der Vergangenheit die Einführung des Studiengangs Mechatronik, dem die Universität sehr viele Studenten verdankt, unter großem Einsatz initiiert. In den letzten Jahren wurden überwiegend stochastische Inhalte unter Einsatz von Computeralgebra-Programmen weiterentwickelt. Diplomarbeiten in der Statistik werden von dieser Abteilung hauptsächlich über industrienahe Themen betreut. In den letzten fünf Jahren haben die Mitglieder der Gruppe nur wenige Arbeiten in einschlägigen Journalen der Stochastik publiziert. Es wird daher von der Kommission angeregt, eine konzeptionelle Zielrichtung in der Forschung für diese Gruppe zu erarbeiten, die die Förderung des wissenschaftlichen Nachwuchses sichert.

\section{Institut für symbolisches Rechnen (RISC)}

Dieses Institut ist außerhalb von Linz in Hagenberg untergebracht. Es genießt national wie international hohes Ansehen, das nicht zuletzt durch den wissenschaftlichen Ruf des Leiters bedingt ist. Die Kommission konnte beim Besuch in Hagenberg einen ausgezeichneten Eindruck von der Führung des Instituts sowie von den wissenschaftlichen Aktivitäten und den Diskussionen unter den Doktoranden und Mitarbeitern gewinnen. Das Institut ist international 
ausgerichtet und rekrutiert seine Doktoranden im Rahmen öffentlicher Ausschreibungen in aller Welt. Dieses Institut ist sicher eine Vorzeigeeinrichtung für die österreichische Mathematik. Die Aktualität der Forschung, die Dynamik des Instituts und die Internationalität sind beeindruckend. Die Finanzierung ist hier gut angelegt und trägt zum weltweiten Renommee der Mathematik in Österreich bei. Im RISC ist eine internationale Graduierten- Schule realisiert, wie die Kommission sie sich vorstellt.

\section{Institut für wissensbasierte mathematische Systeme}

Das Institut ist eng verbunden mit dem Software-Kompetenzzentrum Hagenberg. Auch dieses Institut findet große Anerkennung sowohl innerhalb der Mathematik als auch außerhalb in ihren Anwendungen. Die Kooperationen mit universitären Einrichtungen außerhalb Österreichs sowie mit einschlägigen Industrieunternehmen sind zahlreich. Angesichts der wichtigen Aufgabe, die das Institut im Rahmen des Kompetenzzentrums Hagenberg wahrnimmt, erscheint der Kommission die Ausstattung mit wissenschaftlichen Mitarbeitern eher unterkritisch. Für die Grundlagenforschung wie für die Lehre stehen nur in sehr beschränktem Umfang Mitarbeiter zur Verfügung. Gleichwohl erfüllt das Institut die ihm zugedachten Aufgaben in hohem Maße.

Der mathematische Fachbereich an der Universität Linz wird von der Kommission als eine gelungene Symbiose zwischen Reiner Mathematik, Angewandter Mathematik sowie Industriemathematik angesehen. Besonders hervorzuheben ist die intensive Vernetzung, die viele Synergien freisetzt. Es gibt sicher in Europa nur sehr wenige mathematische Fachbereiche, die ein so klares Profil aufweisen und so erfolgreich bis hinein in wirtschaftliche Anwendungen arbeiten. Die Universität Linz hat zumindest im Sektor der angewandten Mathematik Ausstrahlungskraft weit über Österreich hinaus. 


\subsection{Universität Salzburg}

Die Universität Salzburg verfügt über eine voll ausgebaute Naturwissenschaftliche Fakultät. Es fehlen die Fächer Chemie und Physik. In den letzten Jahren sind durch die Reformdiskussionen neue Impulse für die Hochschule gegeben worden. Dies hat zu einer mehr anwendungsbezogenen Ausbildungsrichtung in der Mathematik geführt. Der Fachbereich Mathematik wurde im Zuge der Strukturreform der Universität im März 2004 neu gegründet und ist mit 13 Stelleninhabern ein sehr kleiner Fachbereich mit flacher Struktur. Die Professoren dieses Fachbereichs sind in Forschung und Lehre gleichberechtigt.

Auf Wünsche der Studenten im Lehrangebot reagiert der Fachbereich entsprechend. Der Kommission erscheint das angebotene Curriculum jedoch noch verbesserungsbedürftig, vor allem in Algebra und Geometrie. Das gegenwärtige Angebot in Geometrie ist zu stark orientiert an dem lokalen Interesse in kombinatorischer Geometrie.

Durch die geringe Größe des Fachbereichs konzentriert sich die Forschung auf die Schwerpunkte Diskrete und Stochastische Geometrie, Metrische Zahlentheorie und Zahlentheoretische Numerik sowie Stochastik und Dynamik, wobei die stochastische Betrachtungsweise die Klammer für alle Bereiche bildet. Die Lehre hat einen sehr hohen Stellenwert. Der Fachbereich arbeitet insgesamt auf hohem Niveau. Die Studenten werden mit speziellen Angeboten angeworben. Grundlage ist eine sehr ausgeprägte Gesprächskultur der Stelleninhaber sowohl untereinander als auch mit den Studierenden. Durch die aktuelle Umstrukturierung ist der relativ kleine Lehrkörper zeitlich sehr stark beansprucht. Für intensive Forschung ist im Augenblick das zeitliche Budget sehr beschränkt.

Innerhalb der nächsten zehn Jahre werden mehr als die Hälfte der jetzigen Planstellen durch Pensionierungen frei. Der Fachbereich versucht bereits jetzt, die Nachbesetzung dieser Stellen mit jüngeren Wissenschaftern vorzubereiten. Die Kommission mahnt an, dass trotz aller Konzentration auf Stochastik als Klammer für die unterschiedlichen Aktivitäten die Reine Mathematik nicht völlig vernachlässigt werden darf. Hierfür muss ausreichend Potential zur Verfügung gestellt werden. Zu denken ist dabei an die Gebiete Algebra oder Analysis. 


\section{Arbeitsgruppe Diskrete Mathematik und Anwendungen}

Das gegenwärtige Forschungsinteresse der kleinen Arbeitsgruppe besteht in Themen zur Kunst und Mathematik sowie zu geometrischen Problemen mit Methoden der Funktionalanalysis. Es werden interessante Themen in den Lehrveranstaltungen angeboten, was sich dann auch in den Themen der Abschlussarbeiten niederschlägt.

\section{Arbeitsgruppe Funktionalanalysis}

Die Arbeitsgruppe ist singulär in der Fakultät. Die Lehrangebote stehen auf hohem Niveau, werden aber nur sehr eingeschränkt nachgefragt. Der Stelleninhaber hat in der Fakultät nur wenige Anknüpfungspunkte. Aufgrund der Isolation gehen von dieser Arbeitsgruppe auch wenige Impulse aus. Das gilt vor allem auch für das Publikationsverhalten.

\section{Arbeitsgruppe Geometrie und Versicherungsmathematik}

Der Leiter dieser Arbeitsgruppe arbeitet auf dem Gebiet der geometrischen Kombinatorik und hier speziell über Konvexität zufälliger Punktmengen. Er hat auf seinem Arbeitsgebiet der Stochastischen Konvexgeometrie relativ wenig publiziert. Die erschienenen Arbeiten lösen jedoch z.T. lange offengebliebene Fragestellung sehr harter Probleme und sind in sehr bekannten Journalen erschienen.

Die Arbeitsgruppe trägt die Masterausbildung in der Versicherungsmathematik und wird von einer Reihe von Führungskräften der Versicherungswirtschaft über Lehraufträge unterstützt. Die vor allem an Wochenenden stattfindenden Kurse zur Ausbildung von Aktuaren sind sehr nachgefragt. Diese Aktivität hat sich zu einem Treffpunkt der Nachwuchskräfte in der Versicherungswirtschaft entwickelt. Masterarbeiten für Studenten werden in diesem Bereich im Wesentlichen von Unternehmen betreut. Wissenschaftlicher Nachwuchs für die Finanzmathematik wird allerdings in Salzburg weniger ausgebildet. Dies geschieht eher an der Technischen Universität Wien.

\section{Arbeitsgruppe Logik und Grundlagenforschung}

Dies ist eine Ein-Mann-Arbeitsgruppe. Der Stelleninhaber beschäftigt sich in erster Linie mit der Modallogik, einem Teilgebiet der Logik, das weltweit nur sehr selten vertreten ist. Die Arbeitsgruppe hat eine enge Zusammenarbeit mit den Salzburger Philosophen aufgebaut. Im 
Rahmen eines zweijährigen FWF-Projektes wurden zwei Promotionen abgeschlossen. Der Stelleninhaber wird in den nächsten Jahren in den Ruhestand treten. Bereits jetzt sollte über die Neubesetzung und Neuausrichtung dieser Professur nachgedacht werden, etwa in Richtung der Reinen Mathematik.

\section{Arbeitsgruppe Monte Carlo- und Quasi-Monte Carlo-Methoden}

Die Arbeitsgruppe beschäftigt sich mit der Konstruktion von Mengen hochdimensionaler Vektoren in allgemeiner Lage, die eine möglichst geringe Diskrepanz im Vergleich mit der Gleichverteilung haben. Die Konstruktion dieser Mengen ist für viele Anwendungen in der Codierungstheorie und numerischen hochdimensionalen Integration von großer Bedeutung. Diese Arbeiten der Gruppe sind in führenden Zeitschriften publiziert worden. Allerdings fällt auf, dass die eingesetzten Verfahren sich recht eng auf lineare Kongruenzmethoden zur Konstruktion solcher Netze beschränken. Dazu gibt es in Österreich eine starke Schule. Ein anderer Teil der Arbeitsgruppe hat sich in der jüngeren Vergangenheit mit der Erzeugung von Zufallszahlen beschäftigt und diese mit statistischen Tests und Diskrepanzmethoden auf Gleichverteilung getestet. Auch diese Arbeiten befinden sich in der österreichischen Tradition und finden eine gewisse nationale und internationale Anerkennung.

Künftig soll die Forschung in Richtung Kryptographie orientiert werden. Mitglieder der Arbeitsgruppe beschäftigen sich auch mit Fragen optimaler Punktverteilung im Einheitskubus. Aufgrund des Bestehens eines Zusammenhangs zu optimalen Codes (über endlichen Körpern) wäre es nach Ansicht der Evaluatoren fruchtbar, grundlegende algebraisch geometrische Konzepte zur Konstruktion optimaler Codes systematisch mit heranzuziehen und weiterzuentwickeln. Methoden z.B. aus der Arithmetischen Algebraischen Geometrie werden bisher noch nicht eingesetzt. Hier wäre eine diesbezügliche Zusammenarbeit mit einer entsprechenden Gruppe von großem Nutzen.

\section{Arbeitsgruppe Numerische Mathematik und Approximationstheorie}

Gegenstand der Forschung ist Klassische Numerische Mathematik und insbesondere Fragestellungen zur Interpolation. Diese Technik spielt in diesem Zusammenhang im internationalen Umfeld jetzt nur noch die Rolle eines Nischenfaches. Es wird daher dringend empfohlen, sich moderneren Feldern der numerischen Mathematik zuzuwenden, wie beispielsweise computernahen Gebieten, wo durchaus eine Verbindung zur Informatik geschaffen werden könnte. 


\section{Arbeitsgruppe Stochastik und Dynamik}

Mit einigen Ausnahmen scheint diese Arbeitsgruppe nur wenige Kontakte zur internationalen Forschung auf hohem Niveau zu besitzen. Die erarbeiteten Ergebnisse werden von der Kommission als teilweise sehr gut bezeichnet. Sie erscheinen auch in sehr guten Zeitschriften dieses Gebietes. Es fällt jedoch auf, dass die Kontakte zu anderen führenden Gruppen, speziell auf dem Gebiet der Dynamischen Systeme in Österreich (z.B. Universität Wien) relativ schwach entwickelt sind. Es fehlen in Österreich offenbar geeignete Plattformen, um die Kommunikation in solchen Gebieten aufrechtzuerhalten und den wissenschaftlichen Nachwuchs auszubilden.

Es soll nochmals betont werden, dass vor allem das Lehramtsstudium an der Universität Salzburg als sehr gut eingeschätzt wird. Vor allem die gute Vernetzung zwischen Fachdidaktik und Pädagogik sollte hervorgehoben werden. Ein Mangel wird jedoch darin gesehen, dass im zweiten Studienabschnitt eine Vertiefung in Mathematik kaum noch möglich ist. 


\subsection{Technische Universität Wien}

Die Fakultät für Mathematik und Geoinformatik setzt sich aus zwei Fachbereichen zusammen: Mathematik und Geometrie. Durch die zunehmenden Hörerzahlen in der Vergangenheit war die Mathematik in den letzten Jahrzehnten kontinuierlich angewachsen. So konnte eine große Zahl an mathematischen Themen angeboten werden. An der Technischen Universität Wien besuchten sämtliche Studierende die Lehrveranstaltungen in Mathematik bzw. Geometrie. Das machte es erforderlich, die Mathematik an der Technischen Universität Wien auch personell relativ gut auszustatten. Durch die Universitätsreform von 2004 muss allerdings auch an dieser Universität bis 2006 drastisch Personal abgebaut werden, und das vor allem im Mittelbau. Der Fakultätsrat hat deshalb beschlossen, die Lehre umzuorganisieren und in der Forschung der Grundlagenforschung höhere Priorität zu geben. Die Fakultät wurde aufgefordert, Schwerpunkte zu setzen und die Vielfalt der Themen zu reduzieren. Von den ursprünglich acht Instituten sind nach der Umstrukturierung nur noch das Institut für Analysis und Scientific Computing, das Institut für Diskrete Mathematik, Geometrie und Algebra und das Institut für Mathematische Wirtschaftsmathematik, Finanz- und Versicherungswesen übriggeblieben.

Es ist nicht zu übersehen, dass sich die Fakultät für Mathematik und Geowissenschaften der Technischen Universität Wien in einem großen Umbruch befindet. Die Kommission hat Zweifel, ob die Mathematik aus sich heraus in angemessener Zeit diesen Umbruch bewältigen kann. Die Gutachter denken deshalb, dass es hilfreich sein könnte, wenn die Universität externe Berater für die Umgestaltung hinzuziehen würde. Dabei sollte allerdings Berücksichtigung finden, dass eine Technische Universität andere Schwerpunkte setzen muss als eine Universität, wobei allerdings auch für eine moderne Ingenieursausbildung moderne Grundlagen der Mathematik unabdingbar sind.

Es fällt auf, dass die Lehrkräfte in ihren Forschungsgebieten vielfach zu sehr auf die Bedürfnisse der eigenen Hochschule fokussiert sind und zu wenig Kontakte nach außen pflegen. Dies dokumentiert sich auch in den Publikationen, die vielfach in wenig bekannten Zeitschriften erscheinen und deren Forschungsinhalte ein wenig verstaubt erscheinen. Eine große Stärke der Technischen Universität Wien liegt in der großen Anzahl junger Wissenschaftler, die mit Enthusiasmus in der Mathematik forschen. Sie bedürfen allerdings noch einer straffen wissenschaftlichen Führung. Dazu sind Hochschullehrer mit Visionen erforderlich, die einen breiten Überblick über die weltweiten Entwicklungen besitzen. 
Die Lehrbelastung der einzelnen Hochschullehrer scheint mit 6-10 Semesterwochenstunden sehr hoch. Es sollte im Zuge der Umorientierung gelingen, an die Technische Universität Wien erstklassige Mathematikprofessoren zu berufen, die zusammen mit den vorhandenen jungen Wissenschaftlern gute Forschungsgruppen aufbauen. Eine voreilige Reduktion des Personals würde im Hinblick darauf völlig falsche Signale senden.

\section{Arbeitsgruppe Computational Statistics}

Diese personell relativ starke Arbeitsgruppe spielt eine wichtige Rolle innerhalb des Ausbildungsspektrums der TU. Sie ist sehr aktiv, was man u.a. an der Publikationshäufigkeit ersehen kann. Die Gruppe sollte jedoch mehr international kooperieren und mit der vorhandenen Finanzmathematik zusammenarbeiten. Die Gruppe beschäftigt sich überwiegend mit Fragen der angewandten Statistik und Methoden der Datenanalyse und deren Anwendungen in Biologie und Medizin. Im Vordergrund stehen dabei Fragen von Fuzzy-Methoden in Statistik und Wahrscheinlichkeitstheorie, Methoden der Ausreißererkennung in der Robusten Statistik sowie Methoden der Datenanalyse und Entwicklung von Software im Kontext der Systeme S bzw. R. Vor allem in den zuletzt genannten Gebieten war die Gruppe äußerst erfolgreich. Die Gruppe ist sehr aktiv in der Ausbildung von Doktoranden und in der Lehre. Ihre Mitglieder haben eine Vielzahl von Vorträgen gehalten und zahlreiche Publikationen in Tagungsbänden und Forschungsberichten publiziert. Im Verhältnis zur Anzahl der Mitglieder ist die geringe Anzahl der Publikationen in führenden Zeitschriften der Statistik und Wahrscheinlichkeitstheorie auffallend, im Gegensatz zu zahlreichen Publikationen in einschlägigen biologischen und medizinischen Journalen. Mit der neuerlichen Wiederbesetzung eines Lehrstuhls und der Neugründung der Gruppe Finanzmathematik hat sich leider eine nicht empfehlenswerte Spaltung der Stochastik in eine sehr angewandte Statistik und einen mathematisch hoch entwickelten Zweig der Stochastik in der Finanzmathematik eingestellt. Hier wäre eine intensive Kooperation in der Forschung und insbesondere in der Ausbildung dringend zu empfehlen. Des weiteren wäre es wünschenswert, dass alle Arbeitsgruppen in einem Gebäude untergebracht werden könnten.

\section{Arbeitsgruppe Computational Neurosciences}

Das Forschungsgebiet sollte in die aktuelle Lehrplanung aufgenommen werden. Die kleine Arbeitsgruppe arbeitet mit sehr guten Resultaten auf dem wichtigen Gebiet der MenschMaschine-Technologie, das weltweit im Aufschwung ist. Dazu müssten in nächster Zeit aller- 
dings die Ressourcen deutlich aufgestockt werden. Gemessen an ihrer Größe betreut die Arbeitsgruppe eine sehr hohe Zahl an Diplomarbeiten. Die Arbeitsgruppe sollte ermutigt werden, ihre wichtigen Ergebnisse patentieren zu lassen und zu kommerzialisieren.

\section{Arbeitsgruppe Simulation von Differentialgleichungen}

Dieses Forschungsgebiet ist an einer Technischen Universität extrem wichtig. Die Arbeitsgruppe ist allerdings viel zu klein. Sie sollte in andere Arbeitsgruppen integriert und personell aufgestockt werden. Ihre methodischen Ziele müssten klarer formuliert werden. Sie beschäftigen sich mit zu vielen Verfahrensproblemen. Auch die internationale Kooperation sollte ausgebaut werden. Um die vielfältigen erfolgversprechenden Aktivitäten in dem zukünftigen Gebiet des Scientific Computing und Optimization in Österreich zu bündeln, wird empfohlen, ein nationales Doktorandenprogramm aufzulegen.

\section{Arbeitsgruppe Partielle Differentialgleichungen und dynamische Systeme}

Die Arbeitsgruppe ist sehr aktiv in der Theorie singulärer Störungen für gewöhnliche Differentialgleichungen und in der kinetischen Transportgleichungstheorie. Die Gruppe macht einen recht dynamischen Eindruck. Sie besteht aus jungen und enthusiastischen Forschern. Allerdings gibt es nur wenige internationale Kontakte. Das Arbeitsgebiet der Gruppe ist relativ eng gefasst und könnte von neuen Themenstellungen profitieren. Der geplante Wechsel eines Arbeitsgruppenleiters an die Universität Wien erscheint der Gutachtergruppe keine ideale Entscheidung zu sein. Vielmehr wird angeregt, die Ko-operation beider Universitäten auf diesem Gebiet zu verstärken.

\section{Arbeitsgruppe Algebra}

Die noch jungen Mitglieder der Arbeitsgruppe machen einen sehr motivierten und wissenschaftlich kompetenten Eindruck. Forschungsschwerpunkte sind Grenzgebiete zur Logik (hier pflegt die Gruppe engen Kontakt zur Universität Wien) und zahlentheoretische Fragestellungen. Am meisten hat die Kommission ein Projekt zur Untersuchung sogenannter Klone in Zusammenarbeit mit der Logik an der Universität Wien überzeugt. Die anderen Forschungsthemen in Codes und Kryptographie erscheinen zur Zeit noch nicht die notwendige Tiefe zu besitzen, können aber eine Perspektive darstellen. Auch für die Zahlentheorie wird empfohlen, sich stärker an internationalen Entwicklungen zu ori- 
entieren. Die Gruppe war erfolgreich in der Einwerbung von Drittmitteln und hat auch viel publiziert, wobei jedoch die meisten Arbeiten in weniger anerkannten Zeitschriften erschienen sind.

\section{Arbeitsgruppe Differentialgeometrie und Geometrische Strukturen}

Die Forschungsthemen reichen von der Algebra bis zur Darstellenden Geometrie. Das Niveau der Publikationen ist gut, was auch am Erscheinen in guten Zeitschriften erkennbar ist. Die Kommission hat den Eindruck, dass vor allem die jungen Mitarbeiter hoch motiviert und wissenschaftlich sehr begabt sind. Die Gruppe könnte von verstärkter internationaler Erfahrung profitieren. Insbesondere für die jüngeren Mitarbeiter wäre es ratsam, mindestens ein Jahr im Ausland zu verbringen. Eine Horizonterweiterung scheint der Gutachtergruppe unbedingt notwendig, damit der Anschluss an moderne Entwicklungen in der Differentialgeometrie gefunden wird.

\section{Arbeitsgruppe Kombinatorik und Algorithmen}

Auch diese Gruppe ist relativ klein und wird von einem Mathematiker geführt, der bisher keine Professur hat. Sie beschäftigt sich mit der mathematischen Analyse von Stochastischen Algorithmen, kombinatorischen Problemen der Zahlentheorie und interessanten Anwendungen in der Diskreten Mathematik, aber auch in der Biologie. Die Gruppe ist international sehr gut eingebunden und publiziert in einschlägigen Zeitschriften der Diskreten Mathematik, der Zahlentheorie und der Theoretischen Informatik. Hier ist anzumerken, dass die Themen digitale Zahlenentwicklung, binäre Suchbäume und Eigenschaften verwandter Stochastischer Algorithmen auf Bäumen mit einem recht traditionellen methodischen Ansatz zur Untersuchung erzeugender Funktion bearbeitet werden, die ihre Wurzeln in der starken österreichischen Tradition in diesen Fragestellungen in der Zahlentheorie und Kombinatorik hat. Es ist sicher empfehlenswert, eine Erweiterung der methodischen Basis in der Mathematik und in der Theoretischen Informatik anzustreben.

\section{Arbeitsgruppe Konvexe und Diskrete Geometrie}

Die Gruppe wird von einem der bekanntesten Professoren an der mathematischen Fakultät geleitet. Leider steht dieser kurz vor der Pensionierung, so dass über Nachfolge nachgedacht werden muss. Die Gruppe beschäftigt sich mit klassischen Fragen aus der Geometrie der Zah- 
len und der konvexen Geometrie und ist international führend auf diesem Gebiet. Es gibt zahlreiche intensive Kontakte zu anderen Zentren der Forschung, wodurch die Mitglieder der Gruppe zahlreiche Einladungen wahrnehmen konnten und Forschungsstipendien erhalten. Die personelle Entwicklung der Arbeitsgruppe steht vor einem Umbruch. Außer dem Leiter wird ein weiteres Mitglied der Gruppe in nächster Zeit in den Ruhestand treten. Es gibt jedoch junge und ausgezeichnete Mathematikerinnen und Mathematiker in dieser Gruppe, die auch einem internationalen Vergleich gut standhalten können. Es wurden harte Probleme gelöst und die entsprechenden Arbeiten mit Preisen ausgezeichnet. Weitere frühere Mitglieder der Gruppe wurden auf Dauerpositionen in- und außerhalb Österreichs berufen. Auf längere Sicht wäre es zu begrüßen, wenn sich diese Gruppe neben der fruchtbaren Interaktion mit der Stochastik verstärkt auch um methodisch relevante neuere Entwicklungen aus anderen Gebieten der Mathematik in der Forschung widmen könnte.

\section{Arbeitsgruppe Geometrische Modellierung und Industrielle Geometrie}

Die Arbeitsgruppe ist in Forschung und Lehre sehr aktiv und macht einen dynamischen Eindruck. In ihrer Forschungsarbeit beschäftigt sie sich mit Fragen der geometrischen Modellierung an der Schnittstelle zwischen Geometrie, numerischer Analysis und Approximationstheorie. Es werden beispielsweise Forschungsbeiträge zur geometrischen Modellierung mit CAD, zur Bildverarbeitung sowie zum Einsatz von geometrischer Modellierung in der Architektur und in der Industrie geleistet.

Die Gruppe hat viele internationale Kontakte zu führenden Zentren auf diesen Forschungsgebieten. Sie ist in nationale und internationale Netzwerke eingebunden. Ihre zahlreichen Publikationen in internationalen Zeitschriften oder als Monographie hat ihr internationale Anerkennung eingebracht.

In der Lehre leistet die Arbeitsgruppe einen erfolgreichen Beitrag einerseits in der Lehramtsausbildung und in der Ausbildung von Ingenieursstudenten in Darstellender Geometrie unter Einbeziehung von modernen CAD-Modulen, andererseits in der Geometrie bei der Ausbildung einer großen Zahl von Architekturstudenten. Die Zusammenarbeit mit dem Architekturfachbereich der TU Wien hat zu verschiedenen gemeinsamen Projekten bis hin zu Aktivitäten in der Industrie geführt. Zusammenfassend ergibt sich für die Arbeitsgruppe in Forschung und Lehre ein sehr positives Bild.

Perspektivisch soll dafür Sorge getragen werden, dass der Kernbestand der Arbeitsgruppe nicht geschwächt wird. Gegebenenfalls sollte man sogar an eine Stärkung denken, um insbesondere die personalintensiven Lehranteile bewältigen zu können. Zusammen mit einem 
kompetenten Partner in der Differentialgeometrie an der TU Wien könnten verstärkt auch Forschungsaktivitäten in Richtung Diskrete Differentialgeometrie generiert werden.

\section{Arbeitsgruppe Funktionalanalysis}

Gegenwärtig beschäftigt sich die Arbeitsgruppe mit Verallgemeinerungen von Branges`'Theorie ganzer Funktionen auf Hilberträumen (mit Anwendungen auf die Zahlentheorie, z.B. Charakterisierung der nichttrivialen Nullstellen der Riemannschen Zetafunktion), mit Untersuchungen des Spektrums gewisser singulärer Operatoren auf Räumen mit nichtdefinitem Skalarprodukt sowie mit Block-Operator-Matrizen.

Die Arbeitsgruppe scheint in ihrer Forschungsarbeit an der TU Wien, aber auch national und international isoliert zu arbeiten. Aufgrund des kürzlichen Ausscheidens des früheren Arbeitsgruppenleiters und der ungeklärten Nachfolgeregelung fehlt der Arbeitsgruppe die Einbindung in ein internationales Forschungsumfeld, in dem an aktuellen Problemen gearbeitet wird. Bei der Nachfolgeregelung sollte deshalb auf angemessene Breite und Aktualität, aber auch auf Internationalität der Forschungsarbeit des neuen Stelleninhabers geachtet werden. Ein Nachfolger im Bereich der Geometrischen Analysis und Spektraltheorie könnte bei entsprechender Hochkarätigkeit diese Funktion erfüllen und überdies die vorhandenen Potentiale nutzen und bündeln.

In der Lehre ist die Arbeitsgruppe im normalen Rahmen in die Lehrtätigkeit an der TU Wien eingebunden.

\section{Forschungsgruppe Finanz- und Versicherungsmathematik}

Die Gruppe bietet ein umfangreiches Programm der Ausbildung im Bereich Finanz- und Versicherungsmathematik einschließlich der Aktuarausbildung an. Die Veranstaltungen werden bei den Studenten sehr stark nachgefragt. Einer der Arbeitsgruppenleiter ist ein auch international führender Vertreter der modernen Finanzmathematik, die sich mit der Stochastik von neuen Derivativen Finanzinstrumenten in Erweiterung der klassischen Finanzmathematik beschäftigt. Der andere Hochschullehrer arbeitet über Stochastische Prozesse, Stochastische Risikobewertungen und Versicherungsmathematik, sodass die Gruppe insgesamt mit ihren Mitarbeitern ein breites Spektrum auf dem Gebiet der Finanzstochastik und ihren Anwendungen abdeckt.

Die Gruppe hat durch bedeutende Auszeichnungen und Forschungspreise hohe Anerkennung für ihre Leistungen erhalten. Die internationale Sichtbarkeit der Gruppe wird besonders durch 
die eingeladenen Vorträge auf internationalen Konferenzen, durch die Rufe und die Berufungen von Mitgliedern der Gruppe auf Dauerstellen an internationalen Zentren der Finanzmathematik deutlich. Längerfristig wäre die Frage der Zusammenarbeit in der Forschung mit dem Institut für Statistik an der TU Wien zu beantworten.

\section{Arbeitsgruppe Mathematical Modeling and Simulation}

Innerhalb dieses Instituts wurde früher in der Optimalsteuerung und Optimierung sowie im wissenschaftlichen Rechnen gearbeitet. Optimierung und Steuerung wird jetzt innerhalb anderer Institute weiterverfolgt. Der Kopf der Arbeitsgruppe Wissenschaftliches Rechnen hat kürzlich einen Ruf nach Deutschland angenommen, so dass diese Position vakant ist. Eine weitere Hochschullehrerin dieser Arbeitsgruppe steht kurz vor der Emeritierung, so dass die weitere Zukunft der Arbeitsgruppe unklar ist. Die Stimmung unter den Mitgliedern scheint daher sehr deprimiert zu sein.

Die zur Zeit (meist von Diplomanden) bearbeiteten Probleme stammen aus der Physiologie, der Mechatronik und der Optimierung. Im Wissenschaftlichen Rechnen werden, finanziert über Industriemittel, einige interessante Projekte aus der Airbusindustrie und der Medizin bearbeitet. Das Institut bildet die meisten Diplomanden aus. Trotzdem entsteht der Eindruck, dass aus den genannten Gründen die Perspektive für diese Arbeitsrichtung an der TU fehlt. Das ist bedauerlich, da an einer Technischen Universität diese Gebiete sehr wichtig wären. Man sollte überprüfen, ob die jetzt getroffene Entscheidung wirklich optimal ist. Möglicherweise könnte durch eine andere Zusammenfassung der Bereiche eine bessere Lösung gefunden werden. In der jetzigen Form wird dieses Institut keine Zukunft haben.

\section{Arbeitsgruppe Mathematische Strukturanalyse und Interpolation}

Diese Gruppe ist sehr klein und müsste von ihrer Thematik her eigentlich zum Institut für Diskrete Mathematik und Geometrie gehören. Es gibt jedoch auch Verbindungen zur Funktionalanalysis. In der Theorie von abstrakten Ringen und Gruppen haben sich die Mitarbeiter einige Verdienste erworben. 


\section{Arbeitsgruppe Computational Logic}

Diese Gruppe ist federführend bei der Gödel-Gesellschaft, einer Vereinigung zur Förderung der Logik. Forschungsmäßig hat sie gute Kontakte zur Informatik, steht der Forschergruppe Logik an der Universität Wien aber eher fern. Die Gruppe hat kein eigenes Forschungsseminar, aber die Veranstaltungen der Gödel-Gesellschaft übernehmen in gewisser Weise diese Funktion. International ist die Gruppe offensichtlich sehr gut vernetzt, organisiert viele Veranstaltungen und ist auch beim Einwerben von Drittmitteln sehr erfolgreich. Die Gruppe leistet gute Arbeit. Ein noch nicht voll ausgeschöpftes Potential scheint im Aufbau enger Kontakte zur Arbeitsgruppe Logik an der Universität Wien zu liegen.

Zusammenfassend sei festgehalten, wie auch aus der Darstellung der einzelnen Forschungsaktivitäten der Gruppen hervorgeht, dass in der Mathematik an der Technischen Universität Wien eine übersichtliche Strukturierung dringend angeraten ist. Die Vereinzelung der Arbeitsgruppen führt zu einer gewissen Isolation und fördert nicht die Synergien, die möglich wären. Ähnliches gilt auch in der Verbindung zur Universität Wien.

Für eine erfolgreiche Umstrukturierung der Mathematik an der TU Wien rät die Kommission auf ein ausgewogenes Verhältnis von moderner grundlagenorientierter zu anwendungsorientierter Forschung und Lehre zu achten. Die gegenwärtigen Pläne der Hochschule müssen nach Meinung der Kommission in dieser Hinsicht nachgebessert werden. 


\subsection{Universität Wien}

Die Fakultät für Mathematik der Universität Wien hat die längste Tradition. Sie reicht bis ins Jahre 1365 zurück und hat im Laufe der Jahrhunderte eine Reihe herausragender Persönlichkeiten als Lehrende und Forschende hervorgebracht. Seit Oktober 2004 bildet das Institut für Mathematik mit dem Institut für Formale Logik eine eigene Fakultät. Seit neun Monaten ist die gesamte Fakultät an einem Standort untergebracht.

Die Fakultät hat gegenwärtig 62 Mitglieder und eine Vielzahl von Mitarbeitern, die aus Drittmitteln finanziert werden. Durch die sehr hohe Fluktuation unter den Mitarbeitern, die Rufe an andere Universitäten und Stellen in der Industrie angenommen haben, hatte die Fakultät im Vergleich zu anderen in Österreich bisher keine Probleme, durch Stellenneubesetzung immer wieder eine Erneuerung von Forschung und Lehre zu erreichen. Durch das neue Universitätsgesetz besteht aber die Problematik, qualifiziertes Personal zu finden.

Die Fakultät für Mathematik ist bemüht, in der mathematischen Forschung ein breites Spektrum in Reiner und Angewandter Mathematik abzudecken. Sie führt in der Lehre, mit Ausnahme der Fächer Chemie und Biologie, den gesamten Servicebereich für andere Fakultäten durch. Intensive Kooperationen bestehen mit dem Erwin-Schrödinger- und dem WolfgangPauli Institut. Der Präsident und einer der Direktoren werden gegenwärtig von der Fakultät gestellt.

Die Fakultät für Mathematik der Universität Wien sollte trotz aller Profilbildung und Fokussierung auch in Zukunft das Fach Mathematik in großer Breite abdecken. Mit ihrem ausgezeichneten Ruf in der Gegenwart, aber auch aus der Vergangenheit heraus, muss die Universität Wien ein Anziehungspunkt für Fachwissenschaftler aus allen Ländern sein. Dass die Universität Wien mit ihrem hervorragenden Ruf für Studenten aus Österreich und darüber hinaus attraktiv ist, muss nicht besonders erwähnt werden. Die Kommission macht sich allerdings große Sorgen, dass aufgrund der neuen Universitätsstruktur gerade die Universität Wien besonders negativ betroffen sein könnte. Die guten jungen Wissenschaftler folgen Rufen an andere Institutionen, und derzeit können keine neuen Mitarbeiter eingestellt werden. Das wird auf Dauer zur Verringerung des guten Angebotes führen und die Qualität der Ausbildung senken. Die Kommission hat zum gegenwärtigen Zeitpunkt keinerlei Kritik an der angebotenen Lehre. Das Angebot ist ausgewogen und wird regelmäßig angeboten. Die Fakultät verfügt derzeit über eine Reihe von exzellenten Wissenschaftlern.

Jedoch scheint die Strategie für die Zukunft nicht klar. Das Ziel, möglichst alle Bereiche der Mathematik abzudecken, hat zu einer Vielzahl kleiner Forschungsgruppen geführt. Hier wird sicher eine Straffung und Zusammenführung erforderlich werden. 


\section{Arbeitsgruppe Algebra und Zahlentheorie}

Für die Leitung der Arbeitsgruppe konnte vor einiger Zeit ein renommierter Mathematiker aus dem Ausland gewonnen werden. Unter seiner Leitung arbeitet die Arbeitsgruppe sehr breit und vor allem mit einem sehr aktuellen Spektrum an Fragestellungen der Algebra und Zahlentheorie auf international anerkanntem Niveau. Der Arbeitsgruppenleiter und einige seiner Mitarbeiter befassen sich mit der Arithmetik algebraischer Gruppen (automorphe Darstellungen, automorphe L-Funktionen, Kohomologie arithmetischer Gruppen, Geometrie von Shimura-Varietäten) und Moduliproblemen (Galois-Darstellungen, p-adische Darstellungen). Ein weiterer Teil der Arbeitsgruppe befasst sich mit der Theorie der Lie-Algebren (Deformationstheorie, kristallographische Gruppen, Kohomologie) und der Theorie algebraischer Gruppen (Reduktionstheorie). Eine dritte Arbeitsrichtung sind Fragenstellungen im Zusammenhang mit der Theorie der (Halb-) Gruppen und der profiniten Gruppen. Eine vierte Arbeitsrichtung ist die Theorie der diophantischen Approximation, Gitterpunktfragen sowie Invariantentheorie von Formen höheren Grades.

Die Forschungsarbeiten des Arbeitsgruppenleiters und einiger seiner Mitarbeiter sind international hoch anerkannt. Insgesamt finden die Arbeiten der Arbeitsgruppe national und international Beachtung. Die Arbeitsgruppe pflegt national und vor allem auch international hervorragende Kooperationen zu den Zentren der Algebra und Zahlentheorie, was durch entsprechende Einladungen und Gastwissenschaftlerbesuche dokumentiert ist. In diesem Zusammenhang ist hervorzuheben, dass der Arbeitsgruppenleiter Direktor des ErwinSchrödinger-Instituts ist. In der Lehre leistet die Arbeitsgruppe Algebra und Zahlentheorie sowohl in der Grundausbildung als auch in einer breit gefächerten Spezialisierungsausbildung einen wesentlichen Beitrag.

\section{Arbeitsgruppe Partielle Differentialgleichungen}

Die Leitung dieser Arbeitsgruppe haben zwei ordentliche Professoren. Beide Professoren haben in der Vergangenheit gemeinsame Arbeiten mit Mathematikern von hohem Ruf veröffentlicht. Angesichts des hohen Renommees und der internationalen Anerkennung dieser beiden Mathematiker erscheint das Arbeitsgebiet, das hier an der Universität Wien vertreten ist, im Hinblick auf die Breite des Gebiets Partielle Differentialgleichungen zu eng gewählt. Bei dem jetzt bearbeiteten Gebiet handelt es sich um das Studium von kinetischen Transportgleichungen mit Anwendungen auf Halbleiter durch besondere Transformationen. Angesicht der enormen Breite und Tiefe des Forschungsgebietes Partielle Differentialgleichungen welt- 
weit rät die Kommission nachdrücklich dazu, das Spektrum der Arbeitsgruppe an der Universität Wien deutlich zu verbreitern. Dies sollte möglich sein, zumal der Gruppe vergleichsweise große Ressourcen zur Verfügung stehen.

Angesichts der gegenwärtigen Besetzungen rät die Evaluationskommission davon ab, den bereits gut vertretenen Ausschnitt der Partiellen Differentialgleichung durch eine weitere Berufung zu verstärken. Die dafür notwendigen Mittel könnten an anderer Stelle mit vielleicht noch größerem Gewinn für den Standort Wien eingesetzt werden, z.B. könnte an eine Neuberufung im Bereich der globalen Analysis mit geometrischer Relevanz gedacht werden. Auf diesem Gebiet sind in den letzten zwei Jahrzehnten bahnbrechende neue Forschungsrichtungen entstanden mit engen Beziehungen zur theoretischen Physik, einer der historisch starken Bereiche der Universität Wien. Das Gebiet gehört inzwischen weltweit zu den wichtigsten Forschungsrichtungen in der Mathematik und ist an allen Spitzen-Universitäten in den USA, Japan, England, Frankreich und vielen anderen Universitäten in Europa vertreten. Es sollte auch an der Universität Wien nicht fehlen. Diese Lücke könnte durch eine hochkarätige Neubesetzung geschlossen werden. Eine enge Vernetzung mit den bereits vorhandenen, starken Forschergruppen bildet ein ausgezeichnetes, attraktives Umfeld. Es sollte eine enge Verbindung mit den Arbeitsgruppen der Ergodentheorie und der dynamischen Systeme angestrebt werden. Natürlich würde eine solche Berufung eine breitere Basis für das Gebiet der partiellen Differentialgleichung in Wien liefern. Das betrachtet die Kommission als eine wichtige Priorität.

\section{Arbeitsgruppe Biomathematik}

Die Arbeitsgruppe ist zahlenmäßig sehr klein. Gleichwohl zählt sie aufgrund des Renommees des Arbeitsgruppenleiters zu den wenigen international sichtbaren Gruppen in der modernen Biomathematik weltweit. Wegen der vielfältigen anderweitigen Verpflichtungen des Arbeitsgruppenleiters gelingt es nicht, eine größere Gruppe aufzubauen. Es steht zu befürchten, dass mit dem Ausscheiden des Arbeitsgruppenleiters die Biomathematik an der Universität Wien nicht mit der angemessenen Priorität weiter verfolgt wird. Das sollte (gemeinsam mit der Fakultät für Biologie) verhindert werden, zumal aus dieser Gruppe sehr guter wissenschaftlicher Nachwuchs hervorgegangen ist, der anderorts gute Positionen bekleidet. Die Biologie stellt nach Meinung vieler Experten große Herausforderungen an die Mathematik, deren erfolgreiche Bearbeitung zu Durchbrüchen in den Lebenswissenschaften führen kann. Es sollte außerdem erreicht werden, dass die Mathematikausbildung der Biologen an der Universität Wien von den Mathematikern übernommen wird. 


\section{Computerorientierte Mathematik}

Die Arbeitsgruppe befasst sich u.a. mit der rigorosen mathematischen Begründung von Algorithmen. Dazu gehören Fehlerabschätzungen mit Hilfe der Intervallarithmetik. Die Gutachter haben sich davon überzeugt, dass dies auf hohem Niveau geschieht. Trotzdem ist dieses Forschungsgebiet heute nicht mehr unbedingt zentral. Es gibt weltweit nur wenige mathematische Fachbereiche, die sich dem etwas exotischen Themenbereich verschrieben haben. Bei eventuell einmal eintretenden personellen Veränderungen sollte man darüber nachdenken, ob hier weiter eine eigenständige Arbeitsgruppe geführt werden muss. Daneben gibt es Aktivitäten in der Statistik und der Quantenphysik. Die geleistete Arbeit macht einen sehr guten Eindruck. Diese Wissenschaftler finden sicher auch in einer anderen Gruppierung ihre Anbindung.

\section{Dynamische Systeme}

Die Forschung in dieser Gruppe konzentriert sich auf die drei Themenbereiche $\operatorname{IR}^{\mathrm{d}}$ - Aktionen, Ergodische Theorie und Harmonische Analysis sowie Intervallabbildungen. Die Forschungsarbeiten sind international sichtbar und auf hohem Niveau. Hervorzuheben ist, dass durch einen früheren Mitarbeiter dieser Gruppe vor kurzem ein Durchbruch zu einem Jahrzehnte lang offenen Problem (Littlewood-Vermutung) erzielt wurde. Dieser frühere Mitarbeiter arbeitet am Institute for Advanced Study in Princeton. Die Kommission empfiehlt der Universität zu prüfen, ob es Möglichkeiten gibt, eventuell in einigen Jahren, ihn zu einer Rückkehr nach Österreich zu bewegen. In Frage käme dazu vielleicht eine vorgezogene Wiederbesetzung der Stelle des derzeitigen Arbeitsgruppenleiters. Dies könnte eine wichtige Maßnahme für die Weiterentwicklung der Forschung an der mathematischen Fakultät der Universität Wien sein.

\section{Arbeitsgruppe Diskrete Mathematik}

Die diskrete Mathematik an der Universität Wien befindet sich gerade im Umbruch, da der Ordinarius ausscheidet und seine Nachfolge noch nicht feststeht. Die Zahl der zu betreuenden Diplomanden und Doktoranden durch die verbliebenen Mitarbeiter scheint uns sehr hoch. Trotz dieser Belastung haben die vorgestellten wissenschaftlichen Resultate der Kommission sehr gut gefallen. Die Gutachtergruppe ist der Meinung, dass mit großem Erfolg an aktuellen 
Themen gearbeitet wurde, insbesondere über Fragen der abzählenden Kombinatorik und der q-Algebra. Die Gruppe ist auch gut in die internationale Forschung eingebunden. Ein noch nicht voll ausgeschöpftes Potential wird in einer Zusammenarbeit mit den Gruppen Algebra und Zahlentheorie und Geometrie/Topologie gesehen, die helfen könnte, für die kombinatorischen Formeln interessante Interpretationen im Rahmen der Darstellungstheorie und der Topologie algebraischer Gruppen zu finden.

\section{Arbeitsgruppe Didaktik der Mathematik}

In der fachdidaktischen Forschung werden im Wesentlichen alle Gebiete der Schulmathematik abgedeckt. Schwerpunkte bilden dabei Untersuchungen zur Grundbildung und zu Grundvorstellungen im Mathematikunterricht, zur Stochastik und Analysis im Mathematikunterricht sowie zum Computer im Mathematikunterricht. Es bestehen zahlreiche nationale und internationale Projekte im Zusammenhang mit der Erstellung von Unterrichtsmaterialien. Darüber hinaus bestehen Kontakte zu führenden Mathematikdidaktikern in Deutschland und in der Schweiz. In der Ausbildung der künftigen Unter- und Oberstufenlehrer leistet die kleine Arbeitsgruppe einen enormen Beitrag. Sie bestreitet 19 Semesterwochenstunden in Fachdidaktik inklusive Schulmathematik sowie die Organisation, Durchführung und Vorbereitung der Schulpraktika. Die Arbeitsgruppe bietet regelmäßig Konservatorien und zahlreiche Lehrerfortbildungsaktivitäten an. Darüber hinaus beteiligt sie sich auch an fachwissenschaftlichen Lehrveranstaltungen, was zu einer idealen Verzahnung von fachwissenschaftlichen und fachdidaktischen Anteilen in der Lehrerbildung führt. Die Arbeitsgruppe beteiligt sich auch aktiv an der Entwicklung von Lehrbüchern für die Schule und von didaktischen Materialien. Darüber hinaus bringt sie ihre Kompetenz bei der Zusammenarbeit mit dem Bildungsministerium ein. Es kann damit gesagt werden, dass sich die Arbeitsgruppe Didaktik der Mathematik sehr erfolgreich an Forschung und Lehre beteiligt und hier ausgezeichnet positioniert ist. Diese Leistung ist umso beeindruckender, da die Gruppe bei der gegenwärtigen personellen Ausstattung sehr oft an ihre Leistungsgrenze kommt. Daher sollte dafür Sorge getragen werden, dass die derzeit vakante Professur hochkarätig besetzt wird und die Arbeitsgruppe möglichst darüber hinaus eine personelle Verstärkung erfährt. 


\section{Institut für Logik}

Das Institut war ein Jahrzehnt lang verwaist, bevor vor etwa fünf Jahren ein tatkräftiger Forscher von großem internationalen Renommee als Direktor gewonnen werden konnte. Das Institut ist die erste Forschungsplattform, die an der Universität Wien im Rahmen der neuen Universitätsstruktur eingerichtet wurde. Es verfügt damit über ein eigenes Budget und ist dem Rektor direkt unterstellt.

In der Forschung liegt der Schwerpunkt auf der Mengenlehre. In der Lehre deckt es die Logik in voller Breite ab. Das Studium der Logik ist neuerdings in den Studiengang Mathematik integriert, was der Kommission sehr sinnvoll erscheint. Von den Veranstaltungen des Instituts profitieren neben der mathematischen Fakultät insbesondere auch die Philosophen und die theoretischen Informatiker der Technischen Universität Wien. Bei der Drittmitteleinwerbung war das Institut in den letzten Jahren außerordentlich erfolgreich, und auch die Zahl der Doktoranden hat merklich zugenommen, so dass für die kommenden Jahre insgesamt weitere Promotionen erwartet werden.

Das Institut hat beste internationale Kontakte und ein intensives Gästeprogramm. Die am Institut geleistete Forschungsarbeit erscheint der Kommission auf höchstem internationalen Niveau, was durch viele Publikationen in ausgezeichneten Zeitschriften und Einladungen zu einschlägigen internationalen Tagungen belegt wird.

\section{Institut für Analysis, Differentialgleichungen und Topologie}

Diese Gruppe beschäftigt sich mit einer eindrucksvollen Vielfalt von Fragen aus der Geometrie und der globalen Analysis. Die Themen erstrecken sich in der Spektraltheorie von Schrödinger-Operatoren über Integrale Systeme, symplektische Geometrie, nicht-lineare Distributionen und verallgemeinerte Twistortheorien bis hin zu Problemen der Bildverarbeitung. Eine kürzlich fertiggestellte Habilitationsschrift beschäftigt sich mit neuen Verbindungen zwischen der Morse-Bott-Theorie und der Reidemeister-Torsion. In all diesen Themenbereichen verfolgen die Forscher der Gruppe originelle und spannende Ideen, die zu einer Vielzahl interessanter Resultate führen, welche in den besten Zeitschriften veröffentlicht werden. Die Gruppe hat enge und fruchtbare Beziehungen zu vielen Wissenschaftlern im Inund Ausland und genießt auch international höchstes Ansehen. Die Position dieser Gruppe sollte auch innerhalb der Universität Wien sichtbar gestärkt werden. 
Anmerkung zum Verhältnis zwischen den Mathematischen Fachbereichen und den anderen Fachbereichen der Universität Wien.

Das Fach Mathematik hat sich mehr und mehr auch zu einem Querschnittsfach entwickelt, das eine Bedeutung in nahezu allen Bereichen der Wissenschaft erlangt hat. Bei der Besetzung fachmathematischer Stellen sollte nach Meinung der Kommission auch dieser Aspekt der Entwicklung angemessen Berücksichtigung finden. 


\section{Anhang}

Tabellarische Zusammenstellung der Ausstattung mathematischer Fachbereiche an den österreichischen Universitäten

\section{a) Personalausstattung}

\begin{tabular}{|c|c|}
\hline TU Graz & $\begin{array}{l}\text { Personal } \\
\text { (aus Haushaltsmitteln) }\end{array}$ \\
\hline Institut für Statistik & $\begin{array}{l}2 \text { Professuren } \\
4 \text { Wiss. Mitarbeiter (davon } 2 \text { auf Zeit) }\end{array}$ \\
\hline Institut für Geometrie & $\begin{array}{l}2 \text { Professuren (1 davon vakant) } \\
9 \text { 1/3 Wiss. Mitarbeiter (davon } 1 \text { 1/3 auf } \\
\text { Zeit) } \\
2 \text { Lehrbeauftragte } \\
2 \text { Nichtwissenschaftler }\end{array}$ \\
\hline $\begin{array}{l}\text { Institut für Math. A: Analysis and Computational } \\
\text { Number Theory }\end{array}$ & $\begin{array}{l}2 \text { Professuren } \\
6 \text { Wiss. Mitarbeiter (davon } 1 \text { auf Zeit) } \\
1 \text { Lehrbeauftragter } \\
1 \text { 1/2 Nichtwissenschaftler } \\
6 \text { 1/2 drittmittelfinanzierte Stellen }\end{array}$ \\
\hline $\begin{array}{l}\text { Institut für Mathematik B: Mathematische. Opti- } \\
\text { mierung, Dynamische Systeme, Diskrete Mathe- } \\
\text { matik }\end{array}$ & $\begin{array}{l}2 \text { Professuren } \\
4 \text { Wiss. Mitarbeiter } \\
1 \text { Nichtwissenschaftler } \\
4 \text { drittmittelfinanzierte Stellen }\end{array}$ \\
\hline $\begin{array}{l}\text { Institut für Mathematik C: Mathematische Struk- } \\
\text { turtheorie }\end{array}$ & $\begin{array}{l}1 \text { Professur } \\
41 / 2 \text { Wiss. Mitarbeiter (davon } 1 \text { 1/2 auf } \\
\text { Zeit) } \\
1 \text { Nichtwissenschaftler } \\
3 \text { drittmittelfinanzierte Stellen }\end{array}$ \\
\hline $\begin{array}{l}\text { Institut für Mathematik D: Numerik und } \\
\text { Partielle Differentialgleichungen }\end{array}$ & $\begin{array}{l}2 \text { Professuren (1 emeritiert, } 1 \text { im Ruhe- } \\
\text { stand) } \\
1 \text { Wiss. Mitarbeiter } \\
\mathbf{1} 1 / 2 \text { Nichtwissenschaftler }\end{array}$ \\
\hline
\end{tabular}




\begin{tabular}{|c|c|}
\hline Universität Graz & $\begin{array}{l}\text { Personal } \\
\text { (aus Haushaltsmitteln) }\end{array}$ \\
\hline Arbeitsgruppe: Algebra und Zahlentheorie & $\begin{array}{l}1 \text { Professur } \\
2 \text { Dozenten } \\
1 \text { Wiss. Mitarbeiter } \\
1 / 2 \text { Bundeslehrer } \\
1 / 2 \text { Nichtwissenschaftler } \\
2 \text { drittmittelfinanzierte Stellen }\end{array}$ \\
\hline Arbeitsgruppe: Analysis & $\begin{array}{l}2 \text { Professuren } \\
2 \text { Dozenten } \\
1 \text { Wiss. Mitarbeiter (ohne Vertrag } \\
1 \text { Nichtwissenschaftler }\end{array}$ \\
\hline $\begin{array}{l}\text { Arbeitsgruppe: Kontrolltheorie, Optimierung und } \\
\text { Wissenschaftliches Rechnen }\end{array}$ & $\begin{array}{l}2 \text { Professuren } \\
6 \text { Dozenten } \\
3 \text { Wiss. Mitarbeiter (davon } 1 \text { als Beam- } \\
\text { ter) } \\
2 \text { Nichtwissenschaftler } \\
7 \text { drittmittelfinanzierte Stellen }\end{array}$ \\
\hline $\begin{array}{l}\text { Arbeitsgruppe: Modellierung und dynamische } \\
\text { Systeme }\end{array}$ & $\begin{array}{l}2 \text { Professuren } \\
\mathbf{3} \text { Dozenten } \\
\mathbf{1} \text { Wiss. Mitarbeiter } \\
\mathbf{1} \text { Nichtwissenschaftler } \\
\mathbf{3} \text { drittmittelfinanzierte Stellen }\end{array}$ \\
\hline
\end{tabular}




\begin{tabular}{|c|c|}
\hline Universität Innsbruck & $\begin{array}{l}\text { Personal } \\
\text { (aus Haushaltsmitteln) }\end{array}$ \\
\hline Institut für Mathematik & $\begin{array}{l}\mathbf{3} \text { Professuren (1 davon vakant) } \\
\mathbf{8} \text { Dozenten } \\
\mathbf{5} \text { Wiss. Mitarbeiter } \\
\mathbf{1} \text { Nichtwissenschaftler } \\
1 \text { drittmittelfinanzierte Stelle }\end{array}$ \\
\hline $\begin{array}{l}\text { Institut für Technische Mathematik, Geometrie } \\
\text { und Bauinformatik }\end{array}$ & $\begin{array}{l}4 \text { Professuren (1 (Kat. I) und } 1 \text { (Kat. II) } \\
\text { davon sind vakant) } \\
4 \text { Dozenten } \\
7 \text { Wiss. Mitarbeiter } \\
3 \text { Nichtwissenschaftler } \\
1 \text { drittmittelfinanzierte bzw. sondermit- } \\
\text { telfinanzierte (1/2) Stelle }\end{array}$ \\
\hline $\begin{array}{l}\text { Institut für Informatik, Arbeitsgruppe Infmath I- } \\
\text { maging }\end{array}$ & $\begin{array}{l}1 \text { Professur } \\
3 \text { 1/2 Wiss. Mitarbeiter } \\
2 \text { Nichtwissenschaftler } \\
4 \text { 1/2 drittmittel- bzw. Sondermittelfi- } \\
\text { nanzierte Stellen }\end{array}$ \\
\hline
\end{tabular}

\section{Universität Linz}

Institut für Algebra

\section{Personal \\ (aus Haushaltsmitteln)}

1 Professur

2 Wiss. Mitarbeiter (davon 2 auf Zeit)

4 Lehrbeauftragte

3/4 Nichtwissenschaftler

3 drittmittelfinanzierte Stellen 


\begin{tabular}{|c|c|}
\hline noch Universität Linz & $\begin{array}{l}\text { Personal } \\
\text { (aus Haushaltsmitteln) }\end{array}$ \\
\hline Institut für Finanzmathematik & $\begin{array}{l}1 \text { Professur } \\
2 \text { Wiss. Mitarbeiter } \\
1 \text { Nichtwissenschaftler } \\
\mathbf{4} \text { drittmittelfinanzierte Stellen }\end{array}$ \\
\hline Institut für Analysis & $\begin{array}{l}2 \text { Professuren } \\
2,5 \text { Wiss. Mitarbeiter (davon } 2 \text { auf Zeit) } \\
\mathbf{2} \text { Nichtwissenschaftler } \\
\mathbf{6} \text { drittmittelfinanzierte Stellen }\end{array}$ \\
\hline Institut für Angewandte Geometrie & $\begin{array}{l}1 \text { Professur (Kat. II) } \\
\mathbf{2 , 5} \text { Wiss. Mitarbeiter } \\
\mathbf{4} \text { drittmittelfinanzierte Stellen }\end{array}$ \\
\hline Institut für Industriemathematik & $\begin{array}{l}1 \text { Professur } \\
5 \text { Wiss. Mitarbeiter } \\
\mathbf{2} \text { Nichtwissenschaftler } \\
\text { (plus } \mathbf{1 6} \text { Stellen auf Zeit) } \\
\mathbf{4} \text { drittmittelfinanzierte Stellen }\end{array}$ \\
\hline Institut für Numerische Mathematik & $\begin{array}{l}1 \text { Professur } \\
4 \text { Wiss. Mitarbeiter } \\
\text { 2Nichtwissenschaftler } \\
11 \text { drittmittelfinanzierte Stellen }\end{array}$ \\
\hline Institut für Stochastik & $\begin{array}{l}1 \text { Professur } \\
\text { 3,75 Wiss. Mitarbeiter } \\
\mathbf{1} \text { Nichtwissenschaftler }\end{array}$ \\
\hline Institut für Symbolisches Rechnen & $\begin{array}{l}1 \text { Professur } \\
\mathbf{2 0} \text { Wiss. Mitarbeiter } \\
\mathbf{5} \text { Nichtwissenschaftler }\end{array}$ \\
\hline $\begin{array}{l}\text { Institut für Wissensbasierte Mathematische Syste- } \\
\text { me }\end{array}$ & $\begin{array}{l}2 \text { Professuren } \\
\text { 6,5 Wiss. Mitarbeiter } \\
\mathbf{4} \text { Nichtwissenschaftler }\end{array}$ \\
\hline
\end{tabular}




\begin{tabular}{|ll|}
\hline Universität Salzburg & $\begin{array}{l}\text { Personal } \\
\text { (aus Haushaltsmitteln) }\end{array}$ \\
Fachbereich Mathematik & $\mathbf{3}$ Professuren (davon 1 Vakanz) \\
& $\mathbf{1 0}$ Wiss. Mitarbeiter \\
$\mathbf{3}$ Nichtwissenschaftler & $\mathbf{3}$ drittmittelfinanzierte Stellen \\
\hline
\end{tabular}

\begin{tabular}{|c|c|}
\hline Technische Universität Wien & $\begin{array}{l}\text { Personal } \\
\text { (aus Haushaltsmitteln) }\end{array}$ \\
\hline Institut für Analysis und Scientific Computing & $\begin{array}{l}2 \text { Professuren } \\
4 \text { Wiss. Mitarbeiter (davon } 2 \text { auf Zeit) }\end{array}$ \\
\hline Institut für Diskrete Mathematik und Geometrie & $\begin{array}{l}2 \text { Professuren (1 davon vakant) } \\
9 \text { 1/3 Wiss. Mitarbeiter (davon } 1 \text { 1/3 auf } \\
\text { Zeit) } \\
2 \text { Lehrbeauftragte } \\
2 \text { Nichtwissenschaftler }\end{array}$ \\
\hline $\begin{array}{l}\text { Institut für Wirtschaftsmathematik } \\
\text { (an der Evaluation beteiligt sich nur die Forschungs- } \\
\text { gruppe Finanz- und Versicherungsmathematik) }\end{array}$ & $\begin{array}{l}2 \text { Professuren } \\
6 \text { Wiss. Mitarbeiter (davon } 1 \text { auf Zeit) } \\
1 \text { Lehrbeauftragter } \\
1 \text { 1⁄2 Nichtwissenschaftler } \\
6 \text { 1/2 drittmittelfinanzierte Stellen }\end{array}$ \\
\hline $\begin{array}{l}\text { Institut für Statistik und Wahrscheinlichkeitstheo- } \\
\text { rie }\end{array}$ & $\begin{array}{l}2 \text { Professuren } \\
4 \text { Wiss. Mitarbeiter } \\
1 \text { Nichtwissenschaftler } \\
4 \text { drittmittelfinanzierte Stellen }\end{array}$ \\
\hline
\end{tabular}




\begin{tabular}{|ll|}
\hline Universität Wien & $\begin{array}{l}\text { Personal } \\
\text { (aus Haushaltsmitteln) }\end{array}$ \\
Institut für Mathematik & $\mathbf{1 1}$ Professuren \\
$\mathbf{3 2}$ Wiss. Mitarbeiter (davon $\mathbf{2 , 5}$ auf \\
Zeit) \\
$\mathbf{5}$ Lehrbeauftragte \\
$\mathbf{1 1} 1 / 2$ Nichtwissenschaftler \\
$\mathbf{4 5}$ drittmittelfinanzierte Stellen \\
$\mathbf{2}$ drittmittelfinanzierte Stellen im \\
Techn. Dienst \\
\hline
\end{tabular}




\section{b) Raumausstattung}

\begin{tabular}{|c|c|}
\hline TU Graz & \\
\hline Institut für Mathematik A & $\begin{array}{l}228,80 \mathrm{~m}^{2} \text { mit } 10 \text { Räumen } \\
1 \text { Bibliothek }\end{array}$ \\
\hline Institut für Mathematik B & 1 Bibliothek \\
\hline Institut für Mathematik C & $\begin{array}{l}170,80 \mathrm{~m}^{2} \text { mit } 9 \text { Räumen } \\
48,50 \mathrm{~m}^{2} 1 \text { Seminarraum (gemeinsam mit Institut } \\
\text { für Mathematik D) } \\
146,45 \mathrm{~m}^{2} \text { für Forschung } \\
1 \text { Fachbibliothek }\end{array}$ \\
\hline Institut für Mathematik D & $143,80 \mathrm{~m}^{2}$ mit 6 Räumen \\
\hline Institut für Geometrie & $\begin{array}{l}260,32 \mathrm{~m}^{2} \\
1 \text { Fachbibliothek }\end{array}$ \\
\hline Institut für Statistik & $\begin{array}{l}266 \mathrm{~m}^{2} \text { mit } 10 \text { Räumen (6 Büros, } 1 \text { Sekretariat, } \\
1 \text { Seminarraum, } 1 \text { Computerlabor, } 1 \text { Bibliothek) }\end{array}$ \\
\hline
\end{tabular}

\begin{tabular}{|ll|}
\hline Universität Graz & \\
Institut für Mathematik und wissenschaftliches & $540 \mathrm{~m}^{2}$ mit 29 Büros \\
& $160 \mathrm{~m}^{2}$ mit 8 Büros für Postdocs, Doktoranden + \\
& Gäste \\
& $370 \mathrm{~m}^{2}$ Bibliothek \\
& 3 Seminarräume für max. 30 Studenten \\
& 1 Seminarraum mit 10 PC-Arbeitsplätzen \\
& 1 Hörsaal für 140 Studenten \\
& \\
\hline
\end{tabular}




\begin{tabular}{|c|c|}
\hline \multicolumn{2}{|l|}{ Universität Innsbruck } \\
\hline \multirow[t]{4}{*}{ Institut für Mathematik } & 23 Arbeitsräume \\
\hline & 1 Computerlabor mit 15 Arbeitsplätzen \\
\hline & 1 Workstationraum mit 3 Arbeitsplätzen \\
\hline & 1 Archivraum \\
\hline
\end{tabular}

\begin{tabular}{|c|c|}
\hline Universität Linz & \\
\hline Institut für Algebra & 4 Büroräume \\
\hline Institut für Analysis & $\begin{array}{l}9 \text { Büroräume } \\
2 \text { Sekretariatsräume }\end{array}$ \\
\hline Institut für Angewandte Geometrie & $\begin{array}{l}3 \text { Büroräume mit 4-5 Arbeitsplätzen } \\
1 \text { Raum mit 2-3 Arbeitsplätzen }\end{array}$ \\
\hline Institut für Industriemathematik & $\begin{array}{l}674 \mathrm{~m}^{2} \text { (mit IMCC und RICAM) } \\
1 \text { Bibliothek }\end{array}$ \\
\hline Institut für Numerische Mathematik & $245,40 \mathrm{~m}^{2}$ mit 12 Büroräumen \\
\hline Institut für Stochastik & 5 Büros \\
\hline Institut für Symbolisches Rechnen & $\begin{array}{l}46 \mathrm{~m}^{2} \text { mit } 3 \text { Räumen auf dem Campus } \\
560 \mathrm{~m}^{2} \text { mit } 20 \text { Räumen auf Schloß Hagenberg }\end{array}$ \\
\hline $\begin{array}{l}\text { Institut für Wissensbasierte Mathematische } \\
\text { Systeme }\end{array}$ & $\begin{array}{l}50 \mathrm{~m}^{2} \text { mit } 3 \text { Räumen } \\
180 \mathrm{~m}^{2} \text { Laboratorium auf Schloß Hagenberg }\end{array}$ \\
\hline
\end{tabular}




\begin{tabular}{|l|}
\hline Universität Salzburg \\
Fachbereich Mathematik \\
\\
$100 \mathrm{~m}^{2}$ Rechnerübungsraum mit 15 PC- \\
Arbeitsplätzen \\
3 Archivräume \\
1 Fakultätsbibliothek \\
3 Netzwerkdrucker \\
\end{tabular}

\section{TU Wien}

Institut für Analysis und Scientific Computing $1186 \mathrm{~m}^{2}$ für Personalräume,

$613 \mathrm{~m}^{2}$ für sonstige Räume

Institut für Diskrete Mathematik und Geometrie $1060 \mathrm{~m}^{2}$ für insgesamt:

41 Personalräume,

8 Seminarräume und

3 Computerräume

Institut für Wirtschaftsmathematik

$324,93 \mathrm{~m}^{2}$ Nutzfläche für

10 Personalräume

$1 \mathrm{kl}$. Seminarraum

1 Serverraum

Institut für Statistik und Wahrscheinlichkeits-

Theorie

$725 \mathrm{~m}^{2}$ Nutzfläche für

17 Personalräume

\section{Universität Wien}

Institut für Mathematik

$2.572 \mathrm{~m}^{2}$ mit 69 Dienstzimmern,

4 Besprechungs- bzw. Prüfungszimmer

5 Sekretariate

3 PC-Labors

5 Archive 


\section{c) Finanzielle Ausstattung}

(k.A. = keine Angaben)

\begin{tabular}{|c|c|c|c|c|c|c|c|}
\hline TU Graz & & & & & & & \\
\hline $\begin{array}{l}\text { Kosten- } \\
\text { kalkulation }\end{array}$ & Jahr & $\begin{array}{l}\text { Inst. f. Sta- } \\
\text { tistik }\end{array}$ & $\begin{array}{l}\text { Inst. f. Geo- } \\
\text { metrie }\end{array}$ & $\begin{array}{l}\text { Inst. f. Math. } \\
\text { A }\end{array}$ & \begin{tabular}{|l} 
Inst. f. Math. \\
B
\end{tabular} & $\begin{array}{l}\text { Inst. f. Math. } \\
\text { C }\end{array}$ & \begin{tabular}{|l} 
Inst. f. Math \\
D
\end{tabular} \\
\hline $\begin{array}{l}\text { laufende Aus- } \\
\text { gaben }\end{array}$ & $\begin{array}{l}2001 \\
2002 \\
2003\end{array}$ & $\begin{array}{l}8.430 \\
5.500 \\
4.000\end{array}$ & $\begin{array}{l}\text { k.A. } \\
\text { k.A. } \\
\text { k.A. }\end{array}$ & $\begin{array}{l}15.299 \\
10.590 \\
10.300\end{array}$ & $\begin{array}{r}13.194 \\
9891 \\
7097\end{array}$ & $\begin{array}{l}7.949 \\
7.785 \\
7.358\end{array}$ & $\begin{array}{l}8.216 \\
8.530 \\
4.310\end{array}$ \\
\hline $\begin{array}{l}\text { Investitionen } \\
\text { Bundesmittel }\end{array}$ & $\begin{array}{l}2001 \\
2002 \\
2003\end{array}$ & $\begin{array}{l}1.250 \\
6.700 \\
2.912\end{array}$ & $\begin{array}{l}10.000 \\
10.000 \\
14.500\end{array}$ & $\begin{array}{l}3.633 \\
4.200 \\
4.800\end{array}$ & $\begin{array}{r}8755 \\
11572 \\
3715\end{array}$ & $\begin{array}{l}7.253 \\
6.778 \\
4.666\end{array}$ & $\begin{array}{r}11.170 \\
305 \\
\text { k.A. }\end{array}$ \\
\hline $\begin{array}{l}\text { Investitionen } \\
\text { Drittmittel }\end{array}$ & $\begin{array}{l}2001 \\
2002 \\
2003\end{array}$ & $\begin{array}{r}-- \\
2.101 \\
2.912\end{array}$ & $\begin{array}{l}\text { k.A. } \\
\text { k.A. } \\
\text { k.A. }\end{array}$ & $\begin{array}{l}\text { k.A. } \\
\text { k.A. } \\
\text { k.A. }\end{array}$ & $\begin{array}{l}\text { k.A. } \\
\text { k.A. } \\
\text { k.A. }\end{array}$ & $\begin{array}{c}1.901 \\
\text { k.A. } \\
\text { k.A. }\end{array}$ & $\begin{array}{l}\text { k.A. } \\
\text { k.A. } \\
\text { k.A. }\end{array}$ \\
\hline $\begin{array}{l}\text { Einnahmen } \\
\text { Bundesmittel }\end{array}$ & $\begin{array}{l}2001 \\
2002 \\
2003\end{array}$ & $\begin{array}{l}8.430 \\
5.500 \\
4.000\end{array}$ & $\begin{array}{r}\text { k.A. } \\
7.500 \\
5.000\end{array}$ & $\begin{array}{r}13.735 \\
12.000 \\
9.450\end{array}$ & $\begin{array}{l}\text { k.A. } \\
\text { k.A. } \\
\text { k.A. }\end{array}$ & $\begin{array}{l}7.994 \\
7.860 \\
6.620\end{array}$ & $\begin{array}{l}8.212 \\
8.000 \\
6.450\end{array}$ \\
\hline $\begin{array}{l}\text { Drittmittel } \\
\text { FWF }\end{array}$ & $\begin{array}{l}2001 \\
2002 \\
2003\end{array}$ & $\begin{array}{r}\text { k.A. } \\
\text { k.A. } \\
\text { k.A. }\end{array}$ & $\begin{array}{l}\text { k.A. } \\
\text { k.A. } \\
\text { k.A. }\end{array}$ & $\begin{array}{l}249.135 \\
242.000 \\
257.000\end{array}$ & $\begin{array}{l}180.000 \\
180.000 \\
180.000\end{array}$ & $\begin{array}{l}21.490 \\
63.473 \\
96.188\end{array}$ & $\begin{array}{l}48.000 \\
48.000 \\
24.000\end{array}$ \\
\hline $\begin{array}{l}\text { Drittmittel } \\
\text { Universität }\end{array}$ & $\begin{array}{l}2001 \\
2002 \\
2003\end{array}$ & $\begin{array}{l}\text { k.A. } \\
\text { k.A. } \\
\text { k.A. }\end{array}$ & $\begin{array}{l}\text { k.A. } \\
\text { k.A. } \\
\text { k.A. }\end{array}$ & $\begin{array}{l}\text { k.A. } \\
\text { k.A. } \\
\text { k.A. }\end{array}$ & $\begin{array}{l}\text { k.A. } \\
\text { k.A. } \\
\text { k.A. }\end{array}$ & $\begin{array}{l}\text { k.A. } \\
\text { k.A. } \\
\text { k.A. }\end{array}$ & $\begin{array}{l}\text { k.A. } \\
\text { k.A. } \\
\text { k.A. }\end{array}$ \\
\hline $\begin{array}{l}\text { sonstige } \\
\text { Drittmittel }\end{array}$ & $\begin{array}{l}2001 \\
2002 \\
2003\end{array}$ & $\begin{array}{r}563 \\
17.356 \\
39.382\end{array}$ & $\begin{array}{c}1.800 \\
1.860 \\
\text { k.A. }\end{array}$ & $\begin{array}{l}\text { k.A. } \\
\text { k.A. } \\
\text { k.A. }\end{array}$ & $\begin{array}{r}150.000 \\
75.000 \\
\text { k.A. }\end{array}$ & $\begin{array}{r}\text { k.A. } \\
\text { k.A. } \\
56.133\end{array}$ & $\begin{array}{l}\text { k.A. } \\
\text { k.A. } \\
\text { k.A. }\end{array}$ \\
\hline
\end{tabular}




\begin{tabular}{|c|c|c|c|c|c|c|}
\hline \multicolumn{7}{|c|}{ Universität Graz } \\
\hline $\begin{array}{l}\text { Kosten- } \\
\text { kalkulation }\end{array}$ & Jahr & \begin{tabular}{|l|} 
Inst. f. Math. \\
und wiss. \\
Rechnen \\
\end{tabular} & \begin{tabular}{|l|} 
AG Algebra \\
u. Zahlen- \\
theorie \\
\end{tabular} & AG Analysis & $\begin{array}{l}\text { AG Kontrolltheorie, } \\
\text { Optimierung u. wiss. } \\
\text { Rechnen }\end{array}$ & \begin{tabular}{|l|} 
AG Modellierung \\
u. dynamische \\
Systeme \\
\end{tabular} \\
\hline $\begin{array}{l}\text { laufende Aus- } \\
\text { gaben }\end{array}$ & $\begin{array}{l}2001 \\
2002 \\
2003 \\
\end{array}$ & $\begin{array}{r}16.406 \\
14.270 \\
10.348 \\
\end{array}$ & $\begin{array}{l}\text { k.A. } \\
\text { k.A. } \\
\text { k.A. }\end{array}$ & $\begin{array}{l}\text { k.A. } \\
\text { k.A. } \\
\text { k.A. }\end{array}$ & $\begin{array}{l}\text { k.A. } \\
\text { k.A. } \\
\text { k.A. }\end{array}$ & $\begin{array}{r}\text { k.A. } \\
\text { k.A. } \\
\text { k.A. }\end{array}$ \\
\hline $\begin{array}{l}\text { Investitionen } \\
\text { Bundesmittel }\end{array}$ & $\begin{array}{l}2001 \\
2002 \\
2003 \\
\end{array}$ & $\begin{array}{r}45.748 \\
76.704 \\
22.260 \\
\end{array}$ & $\begin{array}{l}\text { k.A. } \\
\text { k.A. } \\
\text { k.A. }\end{array}$ & $\begin{array}{l}\text { k.A. } \\
\text { k.A. } \\
\text { k.A. }\end{array}$ & $\begin{array}{l}\text { k.A. } \\
\text { k.A. } \\
\text { k.A. }\end{array}$ & $\begin{array}{l}\text { k.A. } \\
\text { k.A. } \\
\text { k.A. }\end{array}$ \\
\hline $\begin{array}{l}\text { Investitionen } \\
\text { Drittmittel }\end{array}$ & \begin{tabular}{|l|}
2001 \\
2002 \\
2003 \\
\end{tabular} & $\begin{array}{l}\text { k.A. } \\
\text { k.A. } \\
\text { k.A. }\end{array}$ & $\begin{array}{l}\text { k.A. } \\
\text { k.A. } \\
\text { k.A. }\end{array}$ & $\begin{array}{l}\text { k.A. } \\
\text { k.A. } \\
\text { k.A. }\end{array}$ & $\begin{array}{l}\text { k.A. } \\
\text { k.A. } \\
\text { k.A. }\end{array}$ & $\begin{array}{l}\text { k.A. } \\
\text { k.A. } \\
\text { k.A. }\end{array}$ \\
\hline $\begin{array}{l}\text { Einnahmen } \\
\text { Bundesmittel }\end{array}$ & \begin{tabular}{|l|}
2001 \\
2002 \\
2003 \\
\end{tabular} & $\begin{array}{l}\text { k.A. } \\
\text { k.A. } \\
\text { k.A. }\end{array}$ & $\begin{array}{l}\text { k.A. } \\
\text { k.A. } \\
\text { k.A. }\end{array}$ & $\begin{array}{l}\text { k.A. } \\
\text { k.A. } \\
\text { k.A. }\end{array}$ & $\begin{array}{l}\text { k.A. } \\
\text { k.A. } \\
\text { k.A. }\end{array}$ & $\begin{array}{l}\text { k.A. } \\
\text { k.A. } \\
\text { k.A. }\end{array}$ \\
\hline $\begin{array}{l}\text { Drittmittel } \\
\text { FWF }\end{array}$ & \begin{tabular}{|l|}
2001 \\
2002 \\
2003 \\
\end{tabular} & $\begin{array}{l}\text { k.A. } \\
\text { k.A. } \\
\text { k.A. }\end{array}$ & $\begin{array}{r}24.674 \\
13.365 \\
6.505 \\
\end{array}$ & $\begin{array}{r}28.956 \\
28.946 \\
3.405 \\
\end{array}$ & $\begin{array}{r}91.172 \\
116.305 \\
178.570 \\
\end{array}$ & $\begin{array}{r}120.555 \\
105.065 \\
83.378 \\
\end{array}$ \\
\hline $\begin{array}{l}\text { Drittmittel } \\
\text { Universität }\end{array}$ & \begin{tabular}{|l|}
2001 \\
2002 \\
2003 \\
\end{tabular} & $\begin{array}{l}\text { k.A. } \\
\text { k.A. } \\
\text { k.A. }\end{array}$ & $\begin{array}{l}\text { k.A. } \\
\text { k.A. } \\
\text { k.A. }\end{array}$ & $\begin{array}{l}\text { k.A. } \\
\text { k.A. } \\
\text { k.A. }\end{array}$ & $\begin{array}{l}\text { k.A. } \\
\text { k.A. } \\
\text { k.A. }\end{array}$ & $\begin{array}{l}\text { k.A. } \\
\text { k.A. } \\
\text { k.A. }\end{array}$ \\
\hline $\begin{array}{l}\text { sonstige } \\
\text { Drittmittel }\end{array}$ & $\begin{array}{l}2001 \\
2002 \\
2003\end{array}$ & $\begin{array}{l}\text { k.A. } \\
\text { k.A. } \\
\text { k.A. }\end{array}$ & $\begin{array}{l}\text { k.A. } \\
\text { k.A. } \\
\text { k.A. }\end{array}$ & $\begin{array}{r}\text { k.A. } \\
\text { k.A. } \\
3.125\end{array}$ & $\begin{array}{r}5.814 \\
2.250 \\
750 \\
\end{array}$ & $\begin{array}{r}2.134 \\
771 \\
901 \\
\end{array}$ \\
\hline
\end{tabular}

\begin{tabular}{|c|c|c|c|c|}
\hline \multicolumn{5}{|c|}{ Universität Innsbruck } \\
\hline $\begin{array}{l}\text { Kosten- } \\
\text { kalkulation }\end{array}$ & Jahr & Inst. f. Mathematik & $\begin{array}{l}\text { Inst ff. techn. Mathematik, } \\
\text { Geometrie u. Bauinformatik }\end{array}$ & Arbeitsgruppe Infmath Imaging \\
\hline \multirow{3}{*}{$\begin{array}{l}\text { laufende Aus- } \\
\text { gaben }\end{array}$} & 2001 & 17.000 & 22.949 & 105.637 \\
\hline & 2002 & 16.600 & 17.385 & 105.386 \\
\hline & 2003 & 12.500 & 14.654 & 61.625 \\
\hline \multirow{3}{*}{$\begin{array}{l}\text { Investitionen } \\
\text { Bundesmittel }\end{array}$} & 2001 & 47.000 & 73.207 & k.A. \\
\hline & 2002 & 18.000 & 21.879 & k.A. \\
\hline & 2003 & 22.000 & 3.852 & k.A. \\
\hline \multirow{3}{*}{$\begin{array}{l}\text { Investitionen } \\
\text { Drittmittel }\end{array}$} & 2001 & 1.400 & k.A. & k.A. \\
\hline & 2002 & 4.800 & k.A. & k.A. \\
\hline & 2003 & 3.900 & k.A. & k.A. \\
\hline \multirow{3}{*}{$\begin{array}{l}\text { Einnahmen } \\
\text { Bundesmittel }\end{array}$} & 2001 & 63.000 & 98.049 & k.A. \\
\hline & 2002 & 34.000 & 38.670 & k.A. \\
\hline & 2003 & 34.000 & 16.444 & k.A. \\
\hline \multirow{3}{*}{$\begin{array}{l}\text { Drittmittel } \\
\text { FWF }\end{array}$} & 2001 & 10.500 & 42.000 & 145.346 \\
\hline & 2002 & 34.800 & 51.000 & 145.346 \\
\hline & 2003 & 49.500 & 49.000 & 200.149 \\
\hline \multirow{3}{*}{$\begin{array}{l}\text { Drittmittel } \\
\text { Universität }\end{array}$} & 2001 & 5.000 & 201 & k.A. \\
\hline & 2002 & 5.000 & 24 & k.A. \\
\hline & 2003 & 1.700 & 72 & k.A. \\
\hline \multirow{3}{*}{$\begin{array}{l}\text { sonstige } \\
\text { Drittmittel }\end{array}$} & 2001 & k.A. & 1.500 & k.A. \\
\hline & 2002 & k.A. & 4.800 & k.A. \\
\hline & 2003 & k.A. & 20.700 & k.A. \\
\hline
\end{tabular}




\begin{tabular}{|c|c|c|c|c|c|}
\hline \multicolumn{6}{|c|}{ Universität Linz (Teil 1) } \\
\hline $\begin{array}{l}\text { Kosten- } \\
\text { kalkulation }\end{array}$ & Jahr & Inst. f. Algebra & Inst. f. Analysis & $\begin{array}{l}\text { Inst. f. angewandte } \\
\text { Geometrie }\end{array}$ & $\begin{array}{l}\text { Inst. f. Industriema- } \\
\text { thematik }\end{array}$ \\
\hline \multirow{3}{*}{$\begin{array}{l}\text { laufende Aus- } \\
\text { gaben }\end{array}$} & 2001 & 6.740 & 13.000 & 4.000 & 14.918 \\
\hline & 2002 & 6.110 & 13.000 & 4.100 & 14.406 \\
\hline & 2003 & 7.834 & 13.217 & 5.300 & 16.494 \\
\hline \multirow{3}{*}{$\begin{array}{l}\text { Investitionen } \\
\text { Bundesmittel }\end{array}$} & 2001 & 2.108 & 4.000 & 15.000 & 10.123 \\
\hline & 2002 & 6.804 & 3.500 & 14.000 & 112.009 \\
\hline & 2003 & 4.852 & 2.800 & k.A. & 11.047 \\
\hline \multirow{3}{*}{$\begin{array}{l}\text { Investitionen } \\
\text { Drittmittel }\end{array}$} & 2001 & k.A. & k.A. & 16.000 & 582.454 \\
\hline & 2002 & k.A. & 1.600 & 20.000 & 464.784 \\
\hline & 2003 & k.A. & 1.200 & 16.000 & 287.166 \\
\hline \multirow{3}{*}{$\begin{array}{l}\text { Einnahmen } \\
\text { Bundesmittel }\end{array}$} & 2001 & 7.892 & k.A. & k.A. & 25.041 \\
\hline & 2002 & 12.274 & k.A. & k.A. & 126.415 \\
\hline & 2003 & 12.612 & k.A. & k.A. & 27.541 \\
\hline \multirow{4}{*}{$\begin{array}{l}\text { Drittmittel } \\
\text { FWF }\end{array}$} & 2001 & k.A. & 140.000 & 56.000 & 285.940 \\
\hline & 2002 & 70.876 & 140.000 & 76.000 & 288.894 \\
\hline & 2003 & 135.200 & 210.000 & 76.000 & 145.927 \\
\hline & & $\begin{array}{l}241.260 \\
\text { (Sonderprojekt über } 36 \\
\text { Monate) }\end{array}$ & & & \\
\hline \multirow{3}{*}{$\begin{array}{l}\text { Drittmittel } \\
\text { Universität }\end{array}$} & 2001 & k.A. & k.A. & k.A. & 340.524 \\
\hline & 2002 & k.A. & k.A. & 31.000 & 235.066 \\
\hline & 2003 & k.A. & 54.000 & 76.000 & 125.094 \\
\hline \multirow{3}{*}{$\begin{array}{l}\text { sonstige } \\
\text { Drittmittel }\end{array}$} & 2001 & k.A. & k.A. & k.A. & k.A. \\
\hline & 2002 & k.A. & k.A. & k.A. & 953.200 \\
\hline & 2003 & k.A. & k.A. & k.A. & 1.115 .000 \\
\hline
\end{tabular}

\begin{tabular}{|l|l|r|r|r|r|}
\hline \multicolumn{2}{|l|}{ Universität Linz (Teil 2) } \\
$\begin{array}{l}\text { Kosten- } \\
\text { kalkulation }\end{array}$ & Jahr & \multicolumn{2}{l|}{$\begin{array}{l}\text { Inst. f. numerische } \\
\text { Mathematik }\end{array}$} & $\begin{array}{l}\text { Inst. f. Stochastik } \\
\text { Inst. f. Symbolisches } \\
\text { Rechnen }\end{array}$ & $\begin{array}{l}\text { Inst. f. Wissens- } \\
\text { basierte Mathem. Sys- } \\
\text { teme }\end{array}$ \\
\hline laufende Aus- & 2001 & 9.011 & 6.318 & 29.280 & 6.304 \\
gaben & 2002 & 13.132 & 6.664 & 33.964 & 5.739 \\
& 2003 & 11.818 & 5.811 & 30.000 & 7.324 \\
\hline Investitionen & 2001 & 44.207 & 7.710 & 50.363 & 3.852 \\
Bundesmittel & 2002 & 28.330 & 7.700 & 42.755 & 6.690 \\
& 2003 & 19.829 & 5.201 & 7.593 & 5.358 \\
\hline
\end{tabular}




\begin{tabular}{|c|c|c|c|c|c|}
\hline \multicolumn{6}{|c|}{ noch Universität Linz (Teil 2) } \\
\hline Investitionen & 2001 & k.A. & k.A. & k.A. & 15.135 \\
\hline \multirow[t]{2}{*}{ Drittmittel } & 2002 & k.A. & k.A. & k.A. & 11.300 \\
\hline & 2003 & k.A. & k.A. & k.A. & 10.648 \\
\hline Einnahmen & 2001 & 316 & 14.028 & k.A. & 10.923 \\
\hline \multirow[t]{2}{*}{ Bundesmittel } & 2002 & 478 & 14.344 & k.A. & 12.827 \\
\hline & 2003 & 759 & 11.012 & k.A. & 11.778 \\
\hline Drittmittel & 2001 & 224.506 & k.A. & k.A. & k.A. \\
\hline \multirow[t]{2}{*}{ FWF } & 2002 & 324.506 & k.A. & k.A. & k.A. \\
\hline & 2003 & 383.083 & k.A. & k.A. & k.A. \\
\hline Drittmittel & 2001 & 7.921 & k.A. & 403.941 & 406.509 \\
\hline \multirow[t]{2}{*}{ Universität } & 2002 & 2.200 & k.A. & 541.758 & 541.767 \\
\hline & 2003 & 15.500 & k.A. & 580.448 & 393.283 \\
\hline sonstige & 2001 & k.A. & k.A. & k.A. & k.A. \\
\hline \multirow[t]{2}{*}{ Drittmittel } & 2002 & k.A. & k.A. & k.A. & k.A. \\
\hline & 2003 & k.A. & k.A. & k.A. & k.A. \\
\hline
\end{tabular}

\begin{tabular}{|l|l|r|}
\hline \multicolumn{2}{|l|}{ Universität Salzburg } & Fachbereich Mathematik \\
\hline $\begin{array}{l}\text { Kosten- } \\
\text { kalkulation }\end{array}$ & Jahr & 17.935 \\
\hline laufende Aus- & 2001 & 20.940 \\
gaben & 2002 & 24.418 \\
\hline Investitionen & 2003 & 10.915 \\
Bundesmittel & 2002 & 27.560 \\
& 2003 & 12.463 \\
\hline Investitionen & 2001 & k.A. \\
Drittmittel & 2002 & k.A. \\
& 2003 & k.A. \\
\hline Einnahmen & 2001 & k.A. \\
Bundesmittel & 2002 & k.A. \\
& 2003 & k.A. \\
\hline Drittmittel & 2001 & k.A. \\
FW & 2002 & k.A. \\
& 2003 & k.A \\
\hline
\end{tabular}




\begin{tabular}{|c|c|c|}
\hline \multicolumn{3}{|c|}{ noch Universität Salzburg } \\
\hline Drittmittel & 2001 & k.A. \\
\hline \multirow[t]{2}{*}{ FW } & 2002 & k.A. \\
\hline & 2003 & k.A \\
\hline Drittmittel & 2001 & 167.059 \\
\hline \multirow[t]{2}{*}{ Universität } & 2002 & 228.827 \\
\hline & 2003 & 127.574 \\
\hline sonstige & 2001 & 12.718 \\
\hline \multirow[t]{2}{*}{ Drittmittel } & 2002 & 26.413 \\
\hline & 2003 & 45.791 \\
\hline
\end{tabular}

\begin{tabular}{|c|c|c|c|c|c|}
\hline \multicolumn{6}{|l|}{ TU Wien } \\
\hline $\begin{array}{l}\text { Kosten- } \\
\text { kalkulation }\end{array}$ & Jahr & $\begin{array}{l}\text { Inst. f. Analysis } \\
\text { u. Scientific } \\
\text { Computing }\end{array}$ & $\begin{array}{l}\text { Inst. f. Diskrete } \\
\text { Mathematik u. } \\
\text { Geometrie }\end{array}$ & $\begin{array}{l}\text { Forschungsgruppe } \\
\text { Finanz- u. Versiche- } \\
\text { rungsmath. }\end{array}$ & $\begin{array}{l}\text { Inst. f. Statistik u } \\
\text { Wahrscheinlichkeitstheorie }\end{array}$ \\
\hline \multirow{3}{*}{$\begin{array}{l}\text { laufende Aus- } \\
\text { gaben }\end{array}$} & 2001 & 78.955 & 94.500 & 32.616 & 34.426 \\
\hline & 2002 & 81.050 & 94.600 & 26.300 & 22.950 \\
\hline & 2003 & 59.821 & 79.200 & 16.610 & 19.650 \\
\hline \multirow{3}{*}{$\begin{array}{l}\text { Investitionen } \\
\text { Bundesmittel }\end{array}$} & 2001 & 30.196 & 32.800 & 36.772 & 20.261 \\
\hline & 2002 & 180.529 & 20.100 & 37.800 & 10.550 \\
\hline & 2003 & 76.451 & 1.800 & 14.990 & 1.500 \\
\hline \multirow{3}{*}{$\begin{array}{l}\text { Investitionen } \\
\text { Drittmittel }\end{array}$} & 2001 & k.A. & 7.900 & k.A. & k.A. \\
\hline & 2002 & k.A. & 9.000 & 10.000 & k.A. \\
\hline & 2003 & k.A. & 6.800 & k.A. & k.A. \\
\hline \multirow{3}{*}{$\begin{array}{l}\text { Einnahmen } \\
\text { Bundesmittel }\end{array}$} & 2001 & 109.150 .93 & 127.300 & 69.388 & k.A. \\
\hline & 2002 & 261.578 .50 & 114.700 & 64.100 & k.A. \\
\hline & 2003 & 136.271 .90 & 81.000 & 31.600 & k.A. \\
\hline \multirow{3}{*}{$\begin{array}{l}\text { Drittmittel } \\
\text { FWF }\end{array}$} & 2001 & 216.416 & 153.000 & 261.678 & k.A. \\
\hline & 2002 & 482.103 & 265.000 & 246.380 & k.A. \\
\hline & 2003 & 609.416 & 482.000 & 238.312 & 397.335 \\
\hline \multirow{3}{*}{$\begin{array}{l}\text { Drittmittel } \\
\text { Universität }\end{array}$} & 2001 & 80.125 & 146.500 & 3.625 & k.A. \\
\hline & 2002 & 191.077 & 156.700 & 978 & k.A. \\
\hline & 2003 & 115.952 & 48.700 & 3.758 & kA. \\
\hline \multirow{3}{*}{$\begin{array}{l}\text { sonstige } \\
\text { Drittmittel }\end{array}$} & 2001 & 78.964 & k.A. & 25.437 & k.A. \\
\hline & 2002 & 178.873 & k.A. & 47.060 & k.A. \\
\hline & 2003 & 842.697 & k.A. & 101.235 & 146.697 \\
\hline
\end{tabular}




\begin{tabular}{|c|c|c|}
\hline \multicolumn{3}{|c|}{ Universität Wien } \\
\hline $\begin{array}{l}\text { Kosten- } \\
\text { kalkulation }\end{array}$ & Jahr & Fakultät für Mathematik \\
\hline $\begin{array}{l}\text { laufende Aus- } \\
\text { gaben }\end{array}$ & $\begin{array}{l}2001 \\
2002 \\
2003\end{array}$ & $\begin{array}{l}55.237 \\
64.590 \\
52.880\end{array}$ \\
\hline $\begin{array}{l}\text { Investitionen } \\
\text { Bundesmittel }\end{array}$ & $\begin{array}{l}2001 \\
2002 \\
2003\end{array}$ & $\begin{array}{l}60.573 \\
80.000 \\
15.000\end{array}$ \\
\hline $\begin{array}{l}\text { Investitionen } \\
\text { Drittmittel }\end{array}$ & $\begin{array}{l}2001 \\
2002 \\
2003\end{array}$ & $\begin{array}{l}\text { k.A. } \\
\text { k.A. } \\
\text { k.A. }\end{array}$ \\
\hline $\begin{array}{l}\text { Einnahmen } \\
\text { Bundesmittel }\end{array}$ & $\begin{array}{l}2001 \\
2002 \\
2003\end{array}$ & $\begin{array}{l}\text { k.A. } \\
\text { k.A. } \\
\text { k.A. }\end{array}$ \\
\hline $\begin{array}{l}\text { Drittmittel } \\
\text { FWF }\end{array}$ & $\begin{array}{l}2001 \\
2002 \\
2003\end{array}$ & $\begin{array}{r}804.198 \\
730.181 \\
1.195 .660\end{array}$ \\
\hline $\begin{array}{l}\text { Drittmittel } \\
\text { Universität }\end{array}$ & $\begin{array}{l}2001 \\
2002 \\
2003\end{array}$ & $\begin{array}{l}120.903 \\
458.663 \\
381.006\end{array}$ \\
\hline $\begin{array}{l}\text { sonstige } \\
\text { Drittmittel }\end{array}$ & $\begin{array}{l}2001 \\
2002 \\
2003\end{array}$ & $\begin{array}{r}124.100 \\
52.580 \\
354.728\end{array}$ \\
\hline
\end{tabular}




\section{d) Geräteausstattung}

\begin{tabular}{|c|c|}
\hline TU Graz & \\
\hline Institut für Mathematik A & k.A. \\
\hline Institut für Mathematik B & $\begin{array}{l}\text { Workstations } \\
\text { pro Mitarbeiter } 1 \text { PC, } 2 \text { davon mit bis zu } 1 \text { GB RAM } \\
1 \text { Dual-Prozessor-Workstation } \\
1 \text { Alpha-Workstation }\end{array}$ \\
\hline Institut für Mathematik C & $\begin{array}{l}3 \text { Notebooks } \\
12 \text { PCs } \\
7 \text { Drucker } \\
1 \text { Farbdrucker } \\
1 \text { Workstation }\end{array}$ \\
\hline Institut für Mathematik D & k.A. \\
\hline Institut für Geometrie & $\begin{array}{l}10 \text { PCs } \\
3 \text { Laptops } \\
2 \text { Beamer }\end{array}$ \\
\hline Institut für Statistik & $\begin{array}{l}13 \text { PCs mit Vernetzung } \\
2 \text { Scanner } \\
2 \text { Laptops } \\
6 \text { Drucker } \\
1 \text { Beamer }\end{array}$ \\
\hline
\end{tabular}

\begin{tabular}{|l|l|}
\hline \multicolumn{2}{|l|}{ Universität Graz } \\
\hline Institut für Mathematik und wissenschaftliches & 10 PC-Arbeitsplätze \\
Rechnen & 2 Workstations \\
& 5 Hochleistungs-PCs \\
& \\
\hline
\end{tabular}




\begin{tabular}{|ll|}
\hline Universität Innsbruck & \\
Institut für Mathematik & 45 Computerarbeitsplätze \\
& 4 Notebooks \\
& 2 Beamer \\
& 4 Messgeräte für farbmetrische Untersuchungen \\
& \\
\hline
\end{tabular}

\begin{tabular}{|c|c|}
\hline Universität Linz & \\
\hline Institut für Algebra & 1 Linux PC Cluster mit gemeinsamen Server \\
\hline Institut für Analysis & $\begin{array}{l}17 \text { PCs } \\
5 \text { Laptops }\end{array}$ \\
\hline Institut für Angewandte Geometrie & $\begin{array}{l}6 \text { PCs (Linux) } \\
\text { 10 Laptops } \\
\text { 3D-Laserscanner }\end{array}$ \\
\hline Institut für Industriemathematik & 7 PCs und 6 Notebooks für Studenten \\
\hline Institut für Numerische Mathematik & $\begin{array}{l}1 \text { PC und } 1 \text { Laptop } \\
1 \text { PC-Labor mit } 13 \text { Dualprozessoren }\end{array}$ \\
\hline Institut für Stochastik & $5 \mathrm{PCs}$ \\
\hline Institut für Symbolisches Rechnen & $\begin{array}{l}30 \text { Linux PCs } \\
25 \text { Linux Laptops } \\
2 \text { SGI } \\
1 \text { Sun Workstation } \\
3 \text { Abteilungsdrucker } \\
1 \text { Beamer }\end{array}$ \\
\hline $\begin{array}{l}\text { Institut für Wissensbasierte Mathematische Sys- } \\
\text { teme }\end{array}$ & $\begin{array}{l}5 \text { Computerarbeitsplätzen } \\
\text { auf Schloss Hagenberg } 10 \text { Computerarbeitsplätze }\end{array}$ \\
\hline
\end{tabular}




\begin{tabular}{|ll|}
\hline Universität Salzburg & \\
Fachbereich Mathematik & 15 PC-Arbeitsplätzen \\
& 3 Netzwerkdrucker \\
\end{tabular}

\begin{tabular}{|ll|}
\hline TU Wien & \\
Institut Analysis und Scientific Computing & $\begin{array}{l}\text { PC für jedes Zimmer und in Praktikumsräumen ver- } \\
\text { fügbar }\end{array}$ \\
& \\
Institut für Diskrete Mathematik und Geometrie & 1 PC pro Mitarbeiter \\
& 40 PCs für Lehrveranstaltungen \\
& File- und Internetserver \\
& Netzwerke \\
& 5 Netzwerkdrucker \\
& Scanner \\
& \\
& \\
Forschergruppe Finanz- und Versicherungsma- & 1 PC pro Mitarbeiter \\
thematik & 3 PCs für Gäste und Diplomanden \\
& 7 Laptops \\
& File- und Internetserver \\
& Netzwerke \\
& 4 Netzwerkdrucker \\
& Scanner \\
Institut für Statistik und Wahrscheinlichkeits- & Beamer \\
theorie & \\
& \\
& 20 PCs für die Lehre \\
& 5 Notebooks \\
& 1 Kopierer \\
& \\
\hline
\end{tabular}

\begin{tabular}{|l|l|}
\hline Universität Wien & \\
\hline Institut für Mathematik & 238 PCs \\
& 50 High End PCs/Server/Cluster \\
& 35 Notebooks \\
& 15 Drucker \\
& 5 Beamer \\
\hline
\end{tabular}




\title{
Fragebogen
}

zur

\section{EVALUATION VON FORSCHUNG und Lehrprogrammen an den}

\author{
Fachbereichen für Mathematik der \\ Österreichischen Universitäten
}

Zurückzusenden über die ÖMG-Landesvorsitzenden bis 30.9.2004

Auszufüllen von den Fachbereichen als Ganzes für Abschnitt A I und Teil B (unter Mitwirkung von Studienkommission, Studiendekan oä und Studienadministration ), von den Instituten für alle anderen Teile. Dabei wird „Institut“ nicht unbedingt im universitätsrechtlichen Sinn verstanden, sondern als die Einheiten, die nach Definition der jeweiligen Universität (lokal festzulegen) für die Erhebung der forschungsbezogenen Daten herangezogen werden soll: also etwa ein nicht zu großes Institut, eine Abteilung („Lehrstuhl“, „Ordiniriat“), eine nicht zu kleine Forschungsgruppe. „Fachbereich“ ist als Vereinigungsmenge aller dieser Einheiten zu verstehen (auch wenn kein formaler Fachbereich eingerichtet ist).

Stichtag für die Gliederung und den Personalstand: 1.7.2004. Dabei muss darauf geachtet werden, dass etwa bei Publikationsdaten die Personen berücksichtigt werden, die zu diesem Zeitpunkt der jeweiligen Einheit angehören, auch wenn ihre Zuordnung früher eventuell eine andere war, ebenso die inzwischen ausgeschiedenen Personen, die in den Erhebungsjahren der (Vorgänger-)Einheit angehört haben, soweit dies eindeutig feststellbar ist.

Alle Beträge (auch vor der Euro-Umstellung) in € umrechnen! 
Teil A. Forschung, Wissenschaftlicher Nachwuchs

\section{SELBSTVERSTÄNDNIS UND ORGANISATION DES FACHBEREICHS} (vom Fachbereich zu beantworten)

1. Kurzdarstellung der Entwicklung des Fachbereichs (Gründung, Ausbau), seines Profils sowie der organisatorischen und institutionellen Gliederung

2. Darstellung des Forschungsprofils/der Forschungsschwerpunkte. Charakterisierung der Arbeiten. Abgrenzung gegenüber anderen Universitäten (ggf. auch international)

\section{Qualitätssicherung}

3.1 Organisationsformen der Forschung und der internen Zusammenarbeit. Gibt es eine institutsoder lehrstuhlübergreifende Forschungsplanung? (ggf. Angabe der Gremien zur Planung und Gestaltung der Forschungskonzeption und der Forschungsstrukturen)

3.2 Prozess- und Ergebniskontrollen zum Erreichen der Ziele (innerhalb des Fachbereichs, der Institute, an den Lehrstühlen, im Rahmen von Forschungsprojekten)

3.3 Anreize zur Förderung herausragender Leistungen Name, Organisationsform (Institut, Abteilung,..), Homepageadresse der Erhebungseinheit, Name des Leiters (und gegebenenfalls von Leitern von Unterorganisationen)

\section{Personelle und sächliche Ausstattung; Finanzen (von den Instituten zu beantworten)}

1. Personal

1.1. Stellen nach Stellenarten zum Stichtag 1.7.2004

\begin{tabular}{|c|c|c|c|c|}
\hline & $\begin{array}{l}\text { aus } \\
\text { Haushaltsmitteln }\end{array}$ & $\begin{array}{l}\text { aus Sonderpro- } \\
\text { grammen } \\
\text { (inkl. dem Institut } \\
\text { zuzuordnenden } \\
\text { Kompetenzzentren, } \\
\text { CD Labor u.ä }\end{array}$ & $\begin{array}{l}\text { Drittmittelpersonal } \\
\text { inkl. FWF }\end{array}$ & Summe \\
\hline $\begin{array}{l}\text { Personalstellen insgesamt } \\
\text { Davon: } \\
\text { a) für wissenschaftliches Personal } \\
\text { davon: Professoren insgesamt } \\
\text { - Universitätsprofessoren } \\
\text { (Lehrstuhl) } \\
\text { - Sonstige Professoren } \\
\text { übriges wissenschaftl. Personal (incl. } \\
\text { Habilitierter Mittelbau) insg. } \\
\text {-wissenschaftl. Mitarbeiter } \\
\text { - auf Zeit } \\
\text { - auf Dauer } \\
\text { - Lehrkräfte für besondere } \\
\text { Aufgaben }\end{array}$ & & & & \\
\hline $\begin{array}{l}\text { zusätzlich: } \\
\text { Lehrbeauftragte } \\
\text { sonstige (einschl. } \\
\text { Honorarprofessoren, } \\
\text { Privatdozenten etc.) } \\
\text { b) nichtwissenschaftliches Personal } \\
\text { davon: } \quad \text { Sekretariate } \\
\text { Technische Dienste } \\
\text { Verwaltung }\end{array}$ & & & & \\
\hline
\end{tabular}


1.2. Abweichungen zwischen Stellen-Soll und -Ist (nur bzgl. wiss. Personal). Gründe für längerfristige Nicht-Besetzungen von (wissenschaftlichen) Stellen

\subsection{Personalfluktuation:}

a) Zahl der Wissenschaftler, die in den letzten fünf Jahren den Fachbereich verlassen und eine andere Tätigkeit aufgenommen haben (Annahme von Rufen, Abgänge wegen Stellenangeboten etc.)

b) Berufungen und Neuzugänge in den letzten fünf Jahren (mit Angabe der institutionellen Herkunft)

c) Welche Schwierigkeiten bestehen bei Berufungen? Gibt es Schwierigkeiten, erfahrene und qualifizierte Mitarbeiter zu gewinnen oder zu halten?

1.4. Wie beurteilt der Fachbereich seine personelle Ausstattung?

1.5 Gibt es am Fachbereich gemeinsame Vorstellungen zur Ausbildung und Förderung des wissenschaftlichen Nachwuchses? (Promotions- bzw. Habilitationsdauer, Aufgaben von Doktoran- den / Habilitanden am Institut, Zulassungskriterien, Maßnahmen zur Qualitätssicherung, Einbindung in Forschergruppen/Forschungsprojekte, Förderinstrumente).

\section{Finanzen}

2.1. Laufende Ausgaben, Investitionsausgaben, Drittmittel, Verwaltungseinnahmen

\begin{tabular}{|l|l|l|}
\hline \multicolumn{1}{|c|}{ in $€$ laufende Ausgaben } & \multicolumn{1}{c|}{2001} & 2003 \\
Investitionen (Bundesmittel) & & \\
Investitionen (Drittmittel) \\
Einnahmen Bundesmittel \\
Drittmittel über die Universität \\
Drittmittel FWF \\
Sonstige Drittmittel, \\
die organisatorisch nicht über Uni \\
laufen, aber dem Institut zuzuordnen sind \\
wie CD-Labors, Kompetenzzentren (bitte unten erläutern)
\end{tabular}

2.2. Forschungsarbeiten und -projekte, die in den letzten drei Jahren über Drittmittel gefördert wurden (Themen der Projekte, Förderungsdauer, Höhe und Herkunft der Drittmittel)

\section{Ausstattung und Infrastruktur}

3.1. Über welche Ausstattung mit Räumen, Geräten, Nutzflächen für die Forschung verfügt das Institut? 
3.2. Angabe der wichtigsten Infrastruktureinrichtungen und ihre Bedeutung für die Forschung

3.3. Wie beurteilt das Institut die Ausstattung? Wo bestehen besondere Defizite?

\section{FORSCHUNGSERTRAG}

1. Veröffentlichungen und Vorträge

1.1 Erschienene Publikationen des Instituts der letzten drei Jahre (2001 bis 2003) mit genauen bibliographischen Angaben, gegliedert nach

Monographien, Aufsätzen (referiert/nicht referiert), Beiträgen zu Sammelwerken

Von Institutsmitgliedern gehaltene Vorträge gegliedert nach invited/contributed, 2001-2003

Erteilte Patente 2001-2003

1.2. Liste der fünf bis zehn wichtigsten Publikationen des Instituts aus den letzten drei Jahren (mit genauen bibl. Angaben).

1.3 Aus der Forschung hervorgegangene Spin-off Firmen (Name, Themenbereich, Umsatz 2003, Zahl der Arbeitsplätze) 2. Anerkennung von Forschungsleistungen

2.1. Gutachtertätigkeiten von Institutsmitgliedern für FWF, DFG, NSF u.a.

2.2 Sonstige wissenschaftsorganisatorische Tätigkeiten

2.3. Preise, Ehrungen von Institutsmitgliedern (mit Angabe der ausgezeichneten Forschungsleistungen)

2.4 Gastprofessuren, Stipendien für Institutsmitglieder

3. Externe Kooperation

3.1. Teilnahme von Fakultätsmitgliedern an auswärtigen Fachtagungen in den letzten drei Jahren 2001 bis 2003 (Zahl der eingeladenen Vorträge auf Kosten der Einladenden, Zahl der „,contributed papers“)

3.2. Wie oft war das Institut Gastgeber für größere (nationale und internationale) wissenschaftliche Veranstaltungen, für welche?

3.3. Welche nationalen und internationalen Kooperationsbeziehungen unterhält das Institut? (Kooperationsbeziehungen mit anderen Hochschulen, Industrieunternehmen, außeruniversitären Forschungseinrichtungen, innerhalb von EU- Netzwerken)

3.4. Ausländische Gastwissenschaftler, Graduierte, Postdoktoranden am Institut während der letzten drei Jahre

4. Führen Sie hier bitte weitere Punkte kurzgefasst an, die Ihnen noch wichtig erscheinen 


\section{WISSENSCHAFTLICHER NACHWUCHS, FINANZEN (von den Instituten zu be- antworten)}

1. Liste der Promotionen und Habilitationen der letzten drei Jahre: Titel und Gutachter.

2. Zahl der Promotionen in den letzten 5 Jahren

a) nach Ort des Examens (am Institut / extern)

b) nach Arbeitsort der Doktoranden/innen (am Institut/ Graduiertenkolleg / extern)

\begin{tabular}{|c|c|c|c|c|c|}
\hline Promotionen von & 1999 & 2000 & 2001 & 2002 & 2003 \\
\hline \multicolumn{6}{|c|}{ Beschäftigten am Institut } \\
\hline \multicolumn{6}{|l|}{ Stipendiaten } \\
\hline \multicolumn{6}{|l|}{ Externen } \\
\hline Summe & & & & & \\
\hline
\end{tabular}

3. Relation: Zahl der Promotionen je Habilitiertem (1999-2003)

4. Promotionsintensität: Zahl der Promotionen 1999-2003 in Prozent der Zahl der Studienabschlüsse (Diplom, Magister, Staatsprüfungen einschl. Lehramt) 4 Jahre zuvor

5. Durchschnittliche Promotionsdauer (Zeit zwischen Diplom/Examen und Promotion) für die letzten 5 Promotionsjahre

6. Durchschnittliches Alter bei der Promotion für die letzten 5 Promotionsjahrgänge

7. Zahl der Habilitationen in den letzten 5 Jahren

8. Durchschnittliche Habilitationsdauer (Zeit zwischen Promotion und Habilitation) für die letzten 5 Jahre

9. Durchschnittliches Alter zum Zeitpunkt der Habilitation für die letzten 5 Habilitationsjahrgänge

10. Wie und in welchem Umfang werden Doktoranden und Habilitanden in die Lehre einbezogen? Wie werden Nachwuchswissenschaftler auf ihre Aufgaben in der Lehre vorbereitet?

11. Welche Arten des Graduiertenstudiums existieren am Institut (Aufbaustudiengänge, Promotionsstudiengänge, Graduiertenkollegs etc.) 


\section{TEIL B: LEHRE UND STUDIUM}

(über die Studienkommission bzw. Studiendekan, nötigenfalls unter Einbeziehung der Studienadministration, zu beantworten)

\section{STUDIENZIELE, AUSBILDUNGSPROFIL}

\section{Ziele und Konzeption der Studiengänge}

1.1. Übersicht über die angebotenen Studiengänge, Abschlüsse und Lehrleistungen (einschl. Angebote als Nebenfach, Aufbau- und Weiterbildungsstudiengänge; Planstudienzeiten)

1.2. Allgemeine Ziele und konzeptionelle Schwerpunkte (inhaltliches Profil) der grundständigen Studiengänge.

1.3. Regionaler oder länderbezogener Kontext des Studienangebots (Studienprofil, Ausbildungsschwerpunkte im Vergleich zu anderen Hochschulen).

1.4. Wo und wie sind die Studienziele (ggf. für einzelne Studienabschnitte) dokumentiert? Wann wurden diese Ziele formuliert und zuletzt revidiert?

Wie erfolgt die Verständigung über Studienziele und Leistungsstandards? Wie spiegeln sich diese Ziele im tatsächlichen Lehrangebot wider?

1.5. Welche Fähigkeiten und Kompetenzen (Kenntnisse und Fertigkeiten) soll das Studium vermitteln? Was sollen die Absolventen können?

1.6. Welche inhaltlichen Schwerpunktbildungen sind in den Studiengängen (im Hauptstudium) möglich? Welche Veränderungen hat es gegeben, welche sind geplant? (Gründe?)

1.7. In welcher Weise wurden bei der Konzeption der Studiengänge Vorstellungen und Anforderungen potentieller Abnehmer der Absolventen und deren mögliche Berufsfelder berücksichtigt? Welche Kontakte bestehen zu Einstellungsträgern?

1.8. Welche Bedeutung hat der für den Studiengang? Werden regelmäßig Lehrveranstaltungen von Praktikern angeboten? Wird von Studierenden ein größerer Bedarf/ein größeres Interesse an praxisbezogenen Lehrveranstaltungen artikuliert?

1.9. Welche Bedeutung haben die Forschungsschwerpunkte für die Konzeption der Studiengänge und für die Ausbildungsrealität? Wie stark ist die studentische Nachfrage nach Lehrveranstaltungen mit besonderem Forschungsbezug?

1.10 Welche Haltung nimmt das Institut zu den neu zu konzipierenden BA / MA -Studiengängen ein (,Bologna Prozess“)?

1.11 Welche internationalen Programme existieren am Institut? 


\section{RAHMENBEDINGUNGEN VON STUDIUM UND LEHRE}

1. Personelle Ressourcen

1.1. Lehrkapazität

a) Wissenschaftliche Stellen:

\begin{tabular}{|l|l|l|l|}
\hline $\begin{array}{l}\text { Lehrkapazität } \\
\text { (in SWS) }\end{array}$ & \multicolumn{3}{|c|}{ Stellen aus } \\
& Haushaltsmitteln & \multicolumn{1}{|c|}{ Sonderprogrammen } & \multicolumn{1}{c|}{ Summe } \\
\hline 1. & & & \\
2. & & & \\
3. & & & \\
4. & & & \\
$\cdots$ & & & \\
\hline
\end{tabular}

b) Gesamt-Lehrkapazität in Semesterwochenstunden :

c) Tatsächliches Lehrangebot der letzten 5 Jahre bei den Semester (in SWS) nach Grund- und Hauptstudium

d) Zahl der Lehrbeauftragten nach Lehrgebieten und SWS; Anteil der Berufspraktiker

\subsection{Servicebilanz}

Welche Lehrleistungen (in SWS) wurden im letzten Studienjahr (WS und SS) für Studiengänge anderer Institute angeboten bzw. in eigenen Studiengängen von anderen Instituten in Anspruch genommen (einschließlich Weiterbildung, Graduiertenkollegs etc.)

\section{Sachausstattung}

2.1. Laborarbeitsplätze/Großgeräte für die Lehre: Art, Anzahl

\subsection{Computerarbeitsplätze für die Lehre: Art, Anzahl}

\subsection{Bibliothek(en)}

Wie werden Bestand und Verfügbarkeit von Standardtiteln (Mehrfachexemplare, Lehrbuchsammlung) und die Aktualisierung des Bestandes bewertet? Wie werden Bestand und Verfügbarkeit von examensrelevanter Vertiefungsliteratur bewertet? Nutzungsmöglichkeiten elektronischer Dienste: Informationsmedien und -vermittlung

2.4. Gesamteinschätzung: Stärken und Defizite in der räumlichen, finanziellen und sächlichen Ausstattung; Desiderate. 
3. Studienanfänger/innen; Studierende

\subsection{Zahl der Studienanfänger (weiblich/männlich)}

a) nach Studiengängen/angestrebten Abschlüssen (1. Fach- und 1. Hochschulsemester) in den letzten 5 Jahren (SS 1999 bis WS 2002/03 jeweils SS + folgendes WS)

\begin{tabular}{|r|l|l|l|l|l|}
\hline $\begin{array}{l}\text { Studiengang/ } \\
\text { angestrebter Abschluss }\end{array}$ & 1999 & 2000 & 2001 & 2002 & 2003 \\
\hline$\ldots . .5 \mathrm{FS}$ \\
$1 \mathrm{HS}$ \\
$1 \mathrm{FS}$ \\
$1 \mathrm{HS}$
\end{tabular}

b) Ortswechsler: Zugänge/Abgänge nach Studiengängen und Fachsemestern (1993)

3.2. Durchschnittliches Alter der Studienanfänger in den letzten 5 Jahren nach Studiengängen

3.3. Relationen: Zahl der Studienanfänger/innen (I. Fachsemester, Vollzeitäquivalente mit folgenden Gewichtungsfaktoren: Diplom: I; Magister-/Lehramt-Hauptfach: 0,5; Magister-/Lehramt-Nebenfach: $0,25)$

a) je Professorensteile

b) je Stelle Wissenschaftliches Personal

\subsection{Zahl der Studierenden (weiblich/männlich)}

a) nach Studiengängen/angestrebten Abschlüssen und Fachsemestern

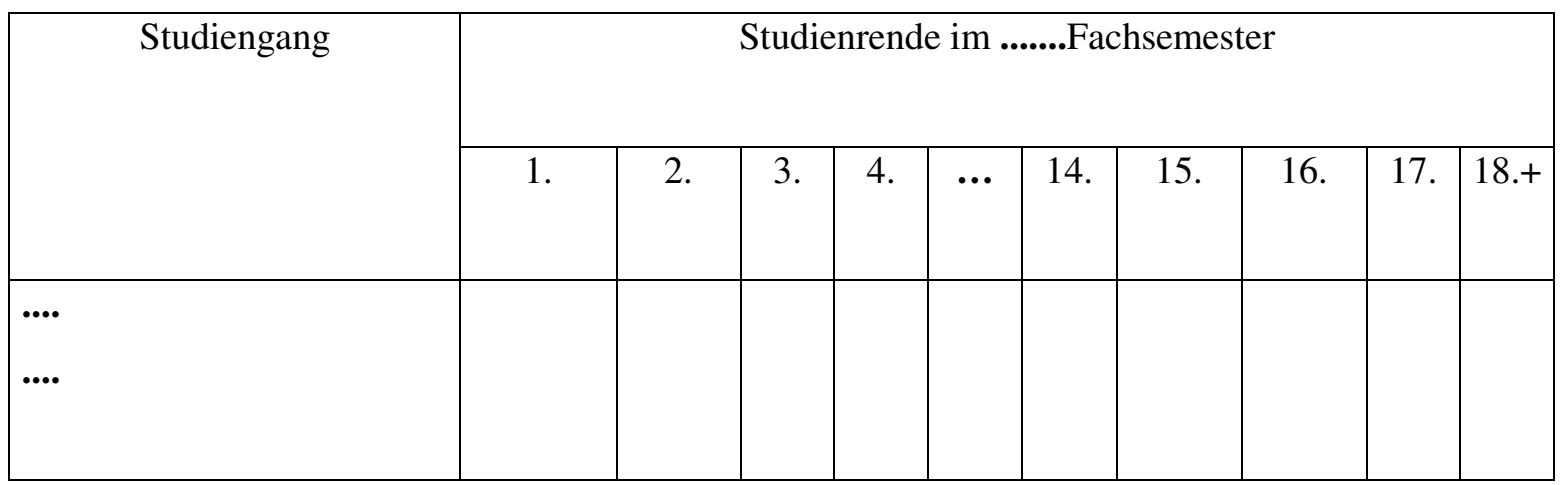

b) Anteile der Studierenden innerhalb der Regelstudienzeit (nach Studiengängen)

3.5. Relationen: Zahl der Studierenden in der Regelstudienzeit (Vollzeitäquivalente, vgl. 3.4.)

a) je Professorensteile

b) je Stelle wissenschaftliches Personal 


\section{STUDIEN-, LEHR- UND PRÜFUNGSORGANISATION}

1. In welchem Maße werden Veranstaltungen des Grundstudiums durch Tutorien/Übungsgruppen begleitet?

2. Relation von Pflicht-, Wahlpflicht- und Wahlveranstaltungen in Grund- und Hauptstudium? Gibt es "Kernfächer" oder "Kernlehrgebiete" des Studiengangs?

3. Gibt es eine fest vorgeschriebene Abfolge der Lehrveranstaltungen? In welcher Weise wird diese für Studierende transparent gemacht (z.B. Studienpläne)?

Gibt es in den zentralen Lehrgebieten ausreichende Wahlmöglichkeiten (Parallelveranstaltungen), die auch unterschiedliche Vermittlungsformen beinhalten?

4. Wie erfolgt die Zuordnung/Verknüpfung der Veranstaltungsangebote zu Prüfungsgebieten (z.B. durch kommentierte Vorlesungsverzeichnisse)

5. Lehrbeiträge der Professoren und des Mittelbaus für Veranstaltungen des Grund- und Hauptstudiums. Wer bestreitet die Einführungsveranstaltungen?

6. Wie und durch wen erfolgt die inhaltliche/zeitliche Planung und Koordination des Lehrangebots? Wie wird die Erbringung des Lehrangebots und die Abdeckung von "Kernfächern" kontrolliert?

7. Studienordnung und Studienrealität: Werden alle in der Studien- und Prüfungsordnung vorgesehene Lehrgebiete regelmäßig angeboten? Probleme und Defizite in der Bereitstellung eines ausreichenden Lehrangebots

8. Internationale Kooperationen in der Lehre (Austauschprogramme, Gastdozent/innen, Praxisaufenthalte, integrierte Studienangebote, internationale Prüfungen etc.)? Wie viele Studierende nehmen teil?

9. Prüfungsverfahren

a) Durchschnittliche Zahl der Wiederholungen bei Prüfungen (Vordiplom, Zwischenprüfung, Diplom, Staatsexamen)

b) Zahl der Prüfungstermine pro Semester. Wie ist die Prüfersuche am Fachbereich organisiert? Hochschule der Studierenden oder Angebot des Fachbereichs/der Fakultät?

c) Dauer und Umfang der Abschlussarbeiten (nach Studiengängen): Zielgröße des Fachbereichs, tatsächliche Größen.

d) Wie lange dauert die gesamte Prüfungsphase im Fach im Regelfall? (Anmeldung, Wartezeiten, Prüfungen, Abschlussarbeit, Begutachtungen)!

e) Regelungen zur Wiederholung von Prüfungen

\section{LEISTUNGSANFORDERUNGEN}

1. Zeitliche Anforderungen an die Studierenden laut Studienordnung (nach Studiengängen; in SWS, nach Grund- und Hauptstudium) Ist der Studiengang unter Berücksichtigung der durchschnittlich benötigten Vor- und Nachbereitungszeit für "normale" Studierende innerhalb der vorgesehenen Planstudienzeit studierbar? 


\section{Leistungsnachweise}

a) Anzahl und Art der in Grund- und Hauptstudium erforderlichen Leistungsnachweise (nach Studiengängen) Wie viele Leistungsnachweise werden von einzelnen Studierenden im Durchschnitt pro Semester faktisch erbracht?

b) In welcher Verbindung stehen die Anforderungen an die Leistungsnachweise mit den Studienzielen?

3. Ausmaß der Erwerbstätigkeit der Studierenden neben dem Studium nach Einschätzung des Fachbereichs/der Fakultät. Wie beurteilt der Fachbereich die Problematik der faktischen Teilzeitstudierenden? (Geschätzter Anteil der Studierenden)

\section{STUDIENERFOLG}

1. Zahl der abgelegten Zwischenprüfungen/Vordiplome der letzten 5 Prüfungsjahre (WS und darauffolgendes SS) nach Studiengängen/Abschlüssen

\begin{tabular}{|l|l|l|l|l|l|}
\hline Studiengang & 1999 & 2000 & 2001 & 2002 & 2003 \\
\hline$\ldots . . . .$. & & & & & \\
\hline$\ldots . . .$. & & & & & \\
\hline
\end{tabular}

2. Zahl der Absolventen/innen der letzten 5 Prüfungsjahre (WS und darauffolgendes SS) nach Studiengängen/Abschlüssen

\begin{tabular}{|l|l|l|l|l|l|}
\hline Studiengang & 1999 & 2000 & 2001 & 2002 & 2003 \\
\hline$\ldots . . . .$. & & & & & \\
\hline..... & & & & & \\
\hline
\end{tabular}

3. Durchschnittliches Alter der Absolventen nach Anfängerjahrgängen/ der letzten 5 Prüfungsjahrgänge (nach Studiengängen)

4. Studienzeiten (nach Studiengängen)

Hinweis: Nach Möglichkeit sollten die folgenden Angaben zum Studienverlauf und zur Studiendauer auf Anfängerkohorten (Anfängerjahrgänge ab 1993; jeweils SS + folgendes WS) bezogen werden. Wenn dies nicht möglich ist, sollten sie auf der Grundlage von Prüfungsjahrgängen (1990, 1995, 1999 - 2003; WS und folgendes SS) erfolgen. 
4.1. Fachstudiendauer bis zum Abschluss der Vordiplom- bzw. Zwischenprüfung (arithmetisches Mittel, Median)

4.2. Fachstudiendauer bei Anmeldung zur Abschlussprüfung (arithmetisches Mittel, Median)

4.3. Fachstudiendauer bei Abschluss des Examens (arithmetisches Mittel, Median, Verteilung)

4.4. Gesamte Verweildauer an der Hochschule (arithmetisches Mittel, Median)

4.5. Anteil der Absolventen innerhalb der Regelstudienzeit

4.6. Studienabbrecher nach Anfängerjahrgängen (1997-2202; wenn nicht möglich: nach Abbruchjahrgängen) und Fachsemesterzahl des Abbruchs (nach Studiengängen)

4.7. Abschlussnoten (Gesamtnoten; nach Anfänger- bzw. Prüfungsjahrgängen) nach Studiengängen

4.8. Prüfungsrücktritte, Prüfungswiederholungen und Ergebnisse von Wiederholungsprüfungen

4.9 Verbleib der Absolventen/innen: Liegen Informationen über den Verbleib der Absolventen vor? Gibt es am Fachbereich Absolventenbefragungen? Welche Maßnahmen hat der Fachbereich ergriffen, um Informationen über den weiteren Werdegang der Absolventen zu gewinnen und/oder mit den Absolventen in Kontakt zu bleiben?

4.10 Wie werden die mit dem Abschluss vermittelten beruflichen Qualifikationen und Arbeitsmarktchancen eingeschätzt?

Anmerkung: Wenn nicht alle diese (insbesondere die statistischen ) Fragen in der verfügbaren Zeit erschöpfend beantwortet werden können, so konzentrieren Sie sich auf die Antworten, die Ihrer Meinung nach ein halbwegs komplettes Bild vermitteln.

\section{QUALITÄTSSICHERUNG}

1. Gibt es Maßnahmen und Verfahren zur Sicherung der Qualität der Lehre und zur Lehrevaluation (z.B. studentische Lehrveranstaltungskritik)? (Kurzdarstellung der Verfahren, ihrer Anlässe und wichtigsten Ergebnisse; Fragebögen etc. bitte beilegen; publizierte Ergebnisse) ?

2. Was unternimmt der Fachbereich zur Verbesserung von Ausbildung und Lehrleistungen?

a) Ist die Situation in der Lehre (quantitativ und qualitativ) Gegenstand regelmäßiger Berichte und Beratungen in Gremien? In welchen?

b) Werden die Angebote zur Studieneingangsphase (Orientierungskurse u.ä.) regelmäßig mit Blick auf die dort gesammelten Erfahrungen überprüft?

c) Wie erfolgt eine Verständigung über Studienziele und Leistungsstandards?

3. Wo sieht der Fachbereich/die Fakultät Defizite in der Vorbildung der Studienanfänger/innen?

Welche Maßnahmen zur Behebung dieser Defizite werden durchgeführt (Vorkurse,

Schreibwerkstätten" u.ä.)? 
4. Worin sieht der Fachbereich/die Fakultät die wesentlichen Gründe für Überschreitungen der Regelstudienzeiten? Wann entstehen diese Verzögerungen im Studienverlauf (Grundstudium bis zur Zwischenprüfung, Hauptstudium)?

5. Welche Faktoren in der Ausstattung des Fachbereichs und in der Organisation von Studium. Lehre und Prüfungen tragen zu einer Studienzeitverlängerung bei? Was sollte und könnte getan werden, um die tatsächlichen Studienzeiten spürbar zu reduzieren?

6. Auf welche Weise wird der Fachbereich/die Fakultät auf "Problemfälle" (z.B. Langzeitstudierende, Prüfungsrücktritte, Studienabbrecher) aufmerksam und wie wird darauf reagiert? Liegen Informationen über die Gründe des Studienabbruchs vor?

7. Welche Bedeutung haben Qualifikationen in der Lehre bei Habilitationen und Berufungen? In welcher Weise werden sie festgestellt und berücksichtigt?

Welche Aktivitäten unternimmt der Fachbereich/die Fakultät zur Förderung der didaktischen Kompetenzen seiner Lehrenden?

\section{GESAMTEINSCHÄTZUNG / BEWERTUNGEN}

1. Stärken und Schwächen der Ausbildung/in den Lehrleistungen (inhaltlich/organisatorisch)

2. Wie wird die Qualität der Ausbildung/Lehre und der Absolventen (auch im Vergleich zu anderen Hochschulen) bewertet?

3. Sind die Leistungsanforderungen angemessen / zu hoch / zu niedrig?

4. Gibt es regelmäßige Engpässe im Lehrangebot? (Welche?)

5. Welches wären wünschenswerte Ergänzungen des Lehrangebots (Veranstaltungsarten, neue Lehrgebiete)?

6. Welches wären praktikable Mittel und Verfahren, um die Qualität der Lehre (und des Studiengangs insgesamt) zu sichern und den Lehr- und Lernbetrieb transparenter zu gestalten?

7. Welche inhaltlichen und organisatorischen Veränderungen wünscht oder plant der Fachbereich/die Fakultät in den nächsten Jahren für Studium und Lehre? 


\section{Liste der Gutachter}

Vorsitzende:

Prof. Dr. Dr. h.c. mult. Karl-Heinz Hoffmann

Forschungszentrum caesar Bonn, Deutschland

Prof. Dr. Jean-Pierre Bourguignon

l'Institut des Hautes Ètudes Scientifiques,

Bures-sur-Yvette, Frankreich

Gutachter:

Prof. Dr. Eva Bayer-Fluckiger

Ecole Polytechnique Federale de Lausanne, Schweiz

Prof. Dr. Friedrich Götze

Universität Bielefeld, Deutschland

Prof. Dr. Jürg Kramer

Humboldt-Universität zu Berlin, Deutschland

Prof. Dr. Pekka Neitaanmäki

University of Jyväskylä, Finland

Prof. Dr. Dietmar Arno Salamon

ETH Zürich, Schweiz

Prof. Dr. Alexander Schrijver

Universiteit van Amsterdam, Netherlands

Prof. Dr. Andreas Schultz

MIT Massachusetts Institute of Technology, USA

Prof. Dr. Wolfgang Soergel

Universität Freiburg, Deutschland

Protokoll:

Margret Schilling

Forschungszentrum caesar Bonn, Deutschland 